\title{
IN SITU DYNAMIC CHARACTERISTICS OF REINFORCED CONCRETE SHEAR WALL BUILDINGS
}

\author{
By \\ Damien Gilles
}

Department of Civil Engineering and Applied Mechanics

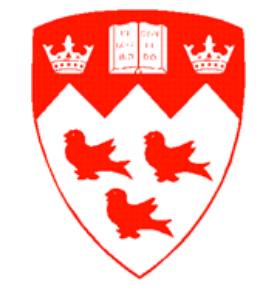

McGill University

Montréal, Québec, Canada

February 2011

A thesis submitted to McGill University in partial fulfillment of the requirements of the degree of Doctor of Philosophy

(C) Damien Gilles, 2011 



\section{Abstract}

Structural engineers routinely need to make assumptions about the dynamic properties (natural periods, mode shapes, and damping) of a building to simulate its response to dynamic loads, such as strong winds or earthquake ground motions. However, the assumed properties may significantly differ from those of the actual building, once it is constructed, due to differences between the idealized model and in situ conditions. The main objectives of this study are to evaluate how common models and assumptions used to predict the dynamic properties of buildings compare to those measured in actual buildings, and to develop improved prediction models.

To this end, ambient vibration measurements were performed in 39 buildings on the island of Montréal and the dynamic properties of up to six vibration modes were identified, for each of these buildings, using the enhanced frequency domain decomposition method. Though the initial goal was to obtain a representative sample of different types of buildings, 27 of these 39 buildings turned out to be reinforced concrete buildings with shear walls providing the main resistance to lateral loads. Hence, the scope of this study was narrowed to the dynamic properties of reinforced concrete shear wall (RCSW) buildings. The measured dynamic properties of this subset of 27 buildings were then used to evaluate different models, proposed in building codes and in the literature, to predict the natural periods and damping characteristics of these types of buildings.

Based on the results of regression analyses, the equation proposed in the 2005 National Building Code of Canada (NBCC 2005) to estimate the fundamental period of RCSW buildings was shown to fit measured period data rather poorly. Alternative equations that incorporate the dimensions of shear walls did not improve the prediction of the fundamental periods, 
despite being more complex. A simple equation was proposed, which matched the measured fundamental period data better. Further, to quantify uncertainty, equations corresponding to the mean, mean minus one standard deviation, and mean plus one standard deviation were produced.

Measured damping values were shown to vary considerably in the different buildings studied, with most values concentrated between one and four percent of critical viscous damping. Different damping models proposed in the literature did not reduce this variability. Based on these observations, as well as past findings that damping increases at large vibration amplitudes, damping values of two percent critical were deemed acceptable for wind design of RCSW buildings, whereas values of three percent were suggested for seismic design.

Finally, simple models to predict the natural periods of torsion and second translation modes were proposed based on regression analyses. These models agree very well with those that have been proposed in the literature. Again, equations corresponding to different probability levels (mean, mean minus one standard deviation, and mean plus one standard deviation) were produced as a measure of uncertainty.

This study should help engineers select realistic values of the dynamic properties of RCSW buildings for structural analysis and design, and should ultimately improve engineers' ability to predict the dynamic response of these buildings. Further, the proposed models could lead to improved recommendations in building codes. 


\section{Résumé}

Pour prédire le comportement d'un bâtiment sous l'effet de différents types de charges dynamiques, telles que les charges de vent et les secousses sismiques, les ingénieurs doivent d'abord estimer les propriétés dynamiques de celui-ci, notamment les périodes naturelles, les déformées modales et l'amortissement. Cependant, ces propriétés peuvent être considérablement différentes de celles du bâtiment réel, une fois construit, dû aux différences entre le modèle idéalisé du bâtiment et les conditions in situ. L'objectif de cette étude est donc d'évaluer les modèles communément utilisés pour estimer les propriétés dynamiques des bâtiments. Plus précisément, le but est de comparer les propriétés estimées à l'aide de ces modèles avec celles mesurées dans des bâtiments existants et de développer de meilleurs modèles, si possible.

À cet effet, des mesures de vibrations ambiantes furent effectuées dans 39 bâtiments sur l'île de Montréal et, pour chacun d'entre eux, les propriétés dynamiques de six modes de vibration furent identifiées à l'aide de la méthode de décomposition dans le domaine des fréquences (FDD). Bien que le but initial fût d'obtenir un échantillon de différents types de bâtiments, 27 des 39 édifices ont une ossature en béton armé et se servent principalement de murs de refend pour résister aux charges latérales. Cette étude se penche donc uniquement sur ce type de bâtiment. Les propriétés dynamiques de ces 27 bâtiments furent utilisées pour évaluer différents modèles proposés dans le Code National du Bâtiment du Canada 2005 (CNB 2005) et dans la littérature scientifique pour estimer les propriétés dynamiques de ce type de bâtiment.

En se basant sur des analyses de régression, cette étude démontre que l'équation proposée dans le CNB 2005 pour estimer la période fondamentale de ce type de bâtiment n'est pas très précise. D'autres 
équations, qui font usage des dimensions des murs de refend, n'offrent pas des estimations plus précises, malgré leur complexité. Une équation simple et plus précise est suggérée pour prédire la période fondamentale. De plus, des équations correspondant à la moyenne moins un écart-type et la moyenne plus un écart-type sont également fournies afin de quantifier l'incertitude associée à l'estimation de la période fondamentale.

Les taux d'amortissement mesurés dans les différents modes de vibration des différents bâtiments furent très variables, avec la plupart des valeurs concentrées entre un et quatre pourcent de l'amortissement critique. Cette variabilité n'est pas réduite si l'on considère d'autres modèles proposés dans la litérature scientifique. En fonction de ces observations, et dû au fait que l'amortissement augmente généralement lors de vibrations de grande amplitude, des valeurs d'amortissement de deux pourcent sont suggérées pour calculer les effets du vent sur les bâtiments avec murs de refend; tandis que et des valeurs de trois pourcent semblent appropriées pour les charges sismiques.

Enfin, des modèles simples, qui concordent très bien avec les modèles suggérés dans la litérature, sont proposés pour estimer les périodes naturelles des modes de torsion et du deuxième mode de translation. De nouveau, des équations correspondant à la moyenne moins un écart-type et la moyenne plus un écart-type sont développées.

Cette étude devrait aider les ingénieurs à sélectionner des valeurs réalistes des propriétés dynamiques pour l'analyse et la conception des bâtiments en béton armé avec murs de refend. En fin de compte, ceci devrait leur permettre de mieux prédire le comportement dynamique de ce type de bâtiment. De plus, les modèles développés dans cette thèse pourraient également mener à des améliorations aux recommendations du Code national du bâtiment du Canada. 


\section{Acknowledgments}

I would like to express my profound and sincere gratitude to my research supervisor, Prof. Ghyslaine McClure, for her attentive guidance and constant encouragement. I feel very fortunate to have had the opportunity to work with her. She has an uncanny ability to make her students feel like colleagues, rather than subordinates. And I benefited tremendously from her knowledge, work ethic, and attention to detail.

This research could not have been possible without the help of many building owners and managers, who allowed our team to perform vibration measurements in their buildings. I would also like to thank the numerous graduate and undergraduate students who were involved in these tests for both their participation and the stimulating discussions that often ensued: Salman Saeed, Jonathan Burke, Arden Heerah, Rebecca Huang, WenChun (Vinci) Huang, Philippe Rosset, Bryce Thomassin, and Helene Tischer.

I would also like to thank the Natural Sciences and Engineering Research Council of Canada (NSERC) for their generous support through the PostGraduate Scholarship and Strategic Networks (Canadian Seismic Research Network) programs. Thanks also to the Vadasz Family Foundation, as well as the Faculty of Engineering and the Department of Civil Engineering at McGill for their generous support. This financial support greatly enhanced my Ph.D. experience by allowing me to focus on my research without the distractions of having to work on the side.

Finally, thanks go to my parents and family for their encouragement and support. Particularly, thanks to my lovely wife, Julie, for being so patient, thoughtful, and caring. 


\section{Table of contents}

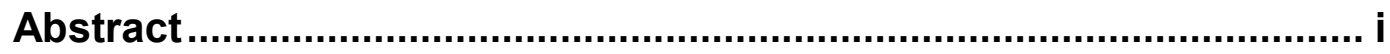

Résumé ......................................................................................... ii

Acknowledgments .................................................................... v

Table of contents .............................................................................. vi

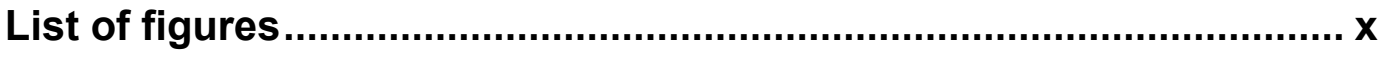

List of tables .......................................................................... xiv

List of symbols ....................................................................... XV

1. Introduction ........................................................................... 1

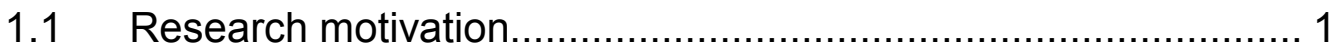

1.2 Research objectives and methodology.............................. 3

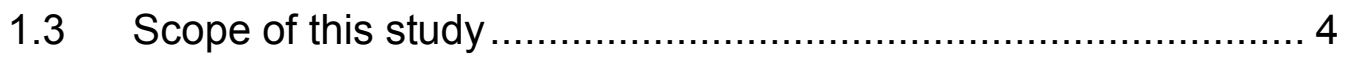

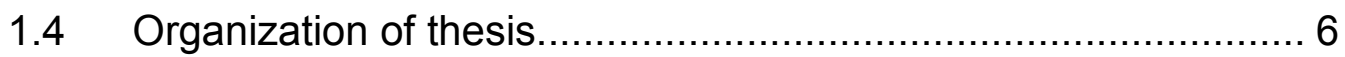

2. Background and literature review............................................. 9

$2.1 \quad$ Structural dynamics concepts ..................................... 9

2.1.1 Dynamic response of a linear SDOF oscillator................ 10

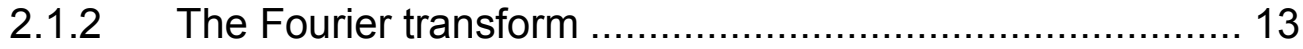

2.1.3 Frequency domain response of a linear SDOF oscillator ... 14

2.1.4 Ground motion and response spectra ....................... 15

2.1.5 Base shear .......................................................... 17

2.1.6 Shear building model for multi-storey buildings............... 18

2.1.7 Dynamic response of a linear MDOF oscillator ................ 20

2.1.8 Frequency domain response of a linear MDOF oscillator .. 22

2.1.9 Response spectrum analysis for a linear MDOF oscillator . 24

2.2 Overview of seismic design provisions in Canada .................. 25

2.2.1 Seismic hazard and the uniform hazard spectrum ........... 26

2.2.2 Analysis methods ................................................. 27

2.2.3 Fundamental period determination.......................... 32

2.3 Dynamic properties of RCSW buildings ......................... 33

2.3.1 Fundamental period ............................................. 34 


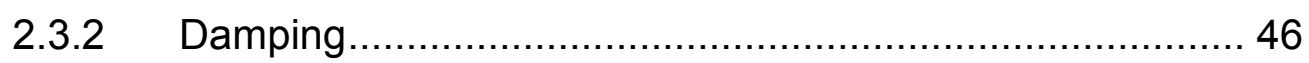

2.3.3 Natural periods of torsion and second translation modes .. 53

2.4 Experimental modal analysis and ambient vibration testing ...... 55

2.4.1 Forced vibration tests ...................................................... 56

2.4.2 Free response tests ................................................... 58

2.4.3 Earthquake response tests ......................................... 59

2.4.4 Ambient vibration tests ............................................... 59

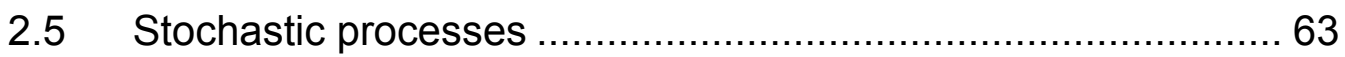

2.5.1 Mean and autocorrelation function .................................. 63

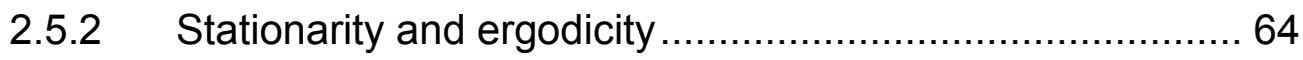

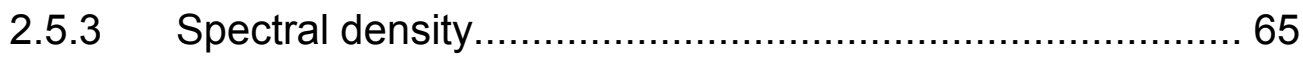

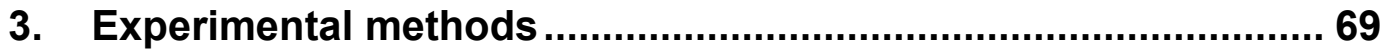

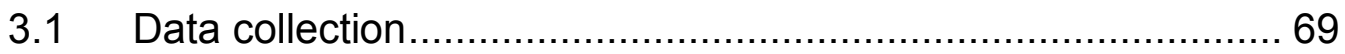

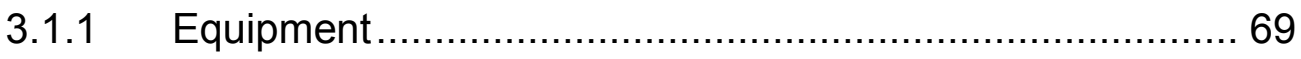

3.1.2 Selection of measurement points .................................. 71

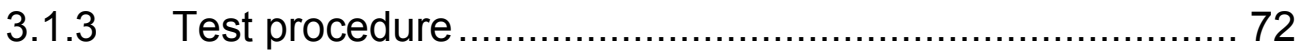

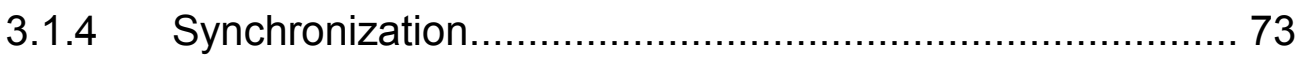

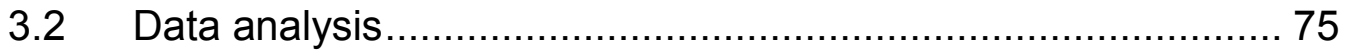

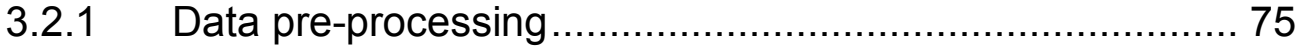

3.2.2 Estimation of modal parameters..................................... 80

4. Validation of experimental methods ............................................. 96

4.1 Modal parameters of model structure ...................................... 96

4.1.1 Description of numerical model ........................................ 96

4.1.2 Theoretical linear modal parameters ................................ 98

4.1.3 Experimental modal parameters ……............................ 99

4.1.4 Comparison of theoretical and experimental modal

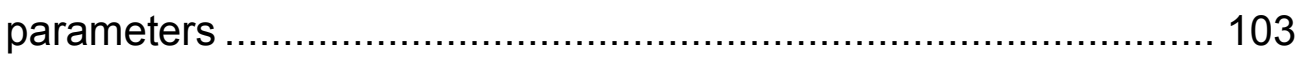

4.2 Modal parameters of real buildings ……............................. 105

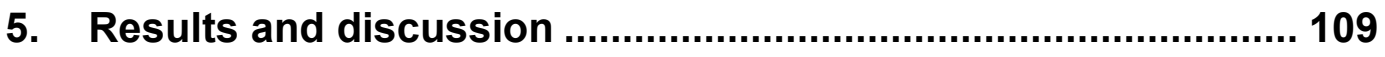

5.1 Fundamental period of RCSW buildings ............................ 109

5.1.1 Measured fundamental period data for RCSW buildings . 110 
5.1.2 Effect of different types of shear walls......................... 112

5.1.3 Comparison with data from other studies ....................... 114

5.1.4 Evaluation of code formula ........................................ 115

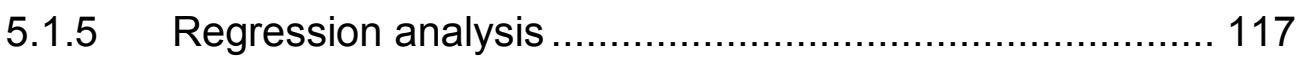

5.1.6 Evaluation of alternative formulae ................................. 124

5.1.7 Improved fundamental period formulae......................... 139

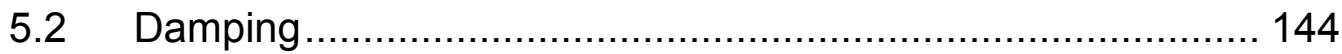

5.2.1 Measured damping in RCSW buildings in Montréal ........ 145

5.2.2 Damping in fundamental translation modes .................... 145

5.2.3 Damping in fundamental torsion mode........................... 149

5.2.4 Damping in higher modes ........................................... 150

5.2.5 Suggested damping values for RCSW buildings .............. 152

5.3 Natural periods of torsion and second translation modes........ 154

5.3.1 Period of fundamental torsion mode ............................. 155

5.3.2 Period of second translation modes ............................... 159

5.3.3 Period of second torsion mode ...................................... 163

6. Conclusions and recommendations ............................................. 166

6.1 Fundamental period of RCSW buildings ............................. 166

6.2 Damping in RCSW buildings ............................................. 169

6.3 Natural periods of torsion and second translation modes........ 170

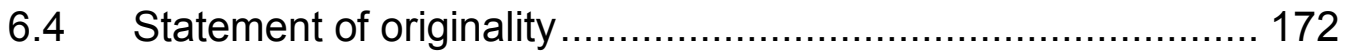

6.5 Limitations and recommendations for future work .................. 174

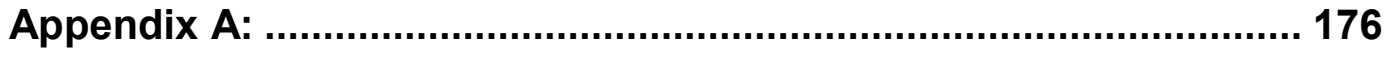

Estimation of damping from logarithmic decrement of autocorrelation function

Appendix B:

Identification of the first two vibration modes of RCSW-1

Appendix C:

Dynamic characteristics of reinforced concrete shear wall buildings in Montréal

Appendix D: 
Measured fundamental periods of shear wall buildings

Appendix E:

Statistical analysis on fundamental period data from buildings with different types of shear walls

Appendix F:

Wall dimensions of selected buildings in Montréal

Appendix G:

Data used for evaluation of alternative fundamental period formulae

References 262 


\section{List of figures}

Figure 2.1: Uniform hazard spectrum for Montréal, adjusted for different soil conditions.

Figure 2.2: Comparison of measured and code fundamental periods

for RCSW buildings in California (Source: Goel and Chopra (1998))

Figure 2.3: Goel and Chopra fundamental period formula.

Figure 2.4: Spectral density estimation using Welch's method.....

Figure 3.1: (a) Typical setup of sensor/DAS pair; (b) Other equipment for ambient vibration tests.

Figure 3.2: Typical selection of measurement points.

Figure 3.3: Excerpt of a typical data file from CityShark II data

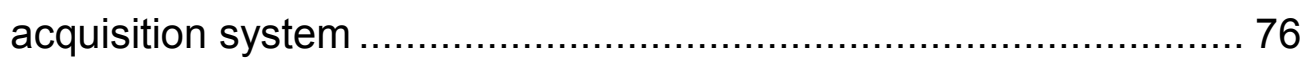

Figure 3.4: Truncation process for data synchronization 78

Figure 3.5: Example of time history records collected at $\mathrm{N}$ collinear

DOF 80

Figure 3.6: Illustration of PSD matrices at discrete frequencies for a

6-DOF system 82

Figure 3.7: Singular value plot with well-separated modes 86

Figure 3.8: Singular value plot with closely-spaced modes 87

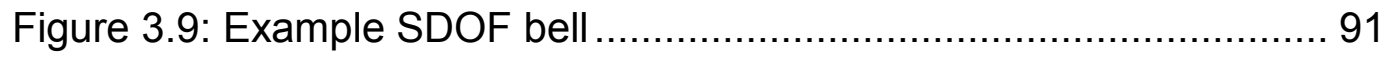

Figure 3.10: Example SDOF autocorrelation function ........................... 93

Figure 3.11: Improved estimate of frequency using zero crossings ......... 93

Figure 3.12: Estimate of viscous damping using logarithmic decrement

Figure 4.1: 6-DOF numerical model used to validate experimental methods.

Figure 4.2: Singular value plot using results from 6-DOF numerical

model. 100

Figure 4.3: SDOF bell for first mode of model structure (load case 1)... 101 
Figure 4.4: SDOF autocorrelation function for first mode of model

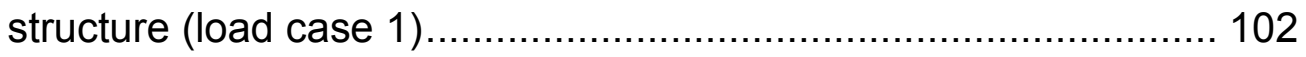

Figure 4.5: Improved estimate of frequency of first mode of model structure (load case 1).

Figure 4.6: Damping estimate for first mode of model structure (load case 1).

Figure 4.7: Comparison of modal frequencies identified on different days 106

Figure 4.8: Comparison of modal damping ratios identified on different days 107

Figure 4.9: Comparison of mode shapes identified on different days .... 108

Figure 5.1: Fundamental periods of buildings in Montréal with different types of shear walls 113

Figure 5.2: Fundamental periods of SW buildings from various studies (DS-2) 115

Figure 5.3: Evaluation of code period formula using measured

fundamental periods of RCSW buildings in Montréal (DS-1)

Figure 5.4: Evaluation of code period formula using measured

fundamental periods of SW buildings (DS-2)

Figure 5.5: Logarithmic plot of measured fundamental periods of

RCSW buildings in Montréal (DS-1) 118

Figure 5.6: Logarithmic plot of measured fundamental periods of SW buildings (DS-2)

Figure 5.7: Results of regression analyses on fundamental period (DS-1)

Figure 5.8: Results of regression analyses on fundamental period (DS-2) 123

Figure 5.9: Plan view of model building to illustrate inconsistency in Goel and Chopra formula

Figure 5.10: 3D view of Building 1 model (Building 2 model almost identical) 
Figure 5.11: Evaluation of Goel and Chopra formula..

Figure 5.12: Evaluation of Goel and Chopra period formula (zoomed).. 132

Figure 5.13: Evaluation of Wallace and Moehle period formula 135

Figure 5.14: Evaluation of Wallace and Moehle period formula (zoomed)

Figure 5.15: Evaluation of Lee et al period formula 137

Figure 5.16: Evaluation of Morales period formula 138

Figure 5.17: Comparison of improved period formulae for RCSW buildings with DS-1

Figure 5.18: Comparison of improved period formulae for RCSW

buildings with DS-2

Figure 5.19: Measured damping ratios of first six vibration modes of

RCSW buildings in Montréal

Figure 5.20: Correlation of damping in fundamental translation mode

with building height for RCSW buildings in Montréal

Figure 5.21: Correlation of damping in fundamental translation mode

with number of storeys for RCSW buildings in Montréal.

Figure 5.22: Correlation of damping in fundamental translation mode

with fundamental frequency for RCSW buildings in Montréal

Figure 5.23: Correlation of damping in fundamental torsion mode

with frequency for RCSW buildings in Montréal

Figure 5.24: Correlation of damping in first six vibration modes with

natural frequency for RCSW buildings in Montréal.

Figure 5.25: Second mode vs fundamental mode damping for RCSW

buildings in Montréal.

Figure 5.26: Correlation of fundamental torsion period with building

height for RCSW buildings in Montréal.

Figure 5.27: Correlation of fundamental torsion period with

fundamental translation period for RCSW buildings in Montréal .... 158

Figure 5.28: Correlation between second translation period and building height for RCSW buildings 161 
Figure 5.29: Correlation between second translation period and corresponding fundamental period for RCSW buildings

Figure 5.30: Correlation between second torsion period and building height for RCSW buildings 164

Figure 5.31: Correlation between second torsion period and fundamental torsion period for RCSW buildings. 165 


\section{List of tables}

Table 3.1: Technical specifications of Lennartz LE-3D/5s sensors.......... 70

Table 4.1: Modal parameters of 6-DOF numerical model....................... 98

Table 4.2: 6-DOF numerical model - Load cases and response DOF .... 99

Table 4.3: Modal parameters of model structure obtained from EFDD.. 103

Table 4.4: Comparison of theoretical and experimental modal

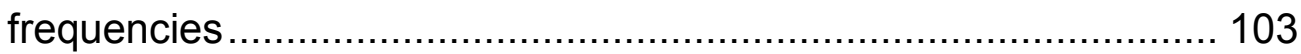

Table 4.5: Comparison of theoretical and experimental modal damping ratios

Table 4.6: Comparison of theoretical and experimental mode shapes (EFDD)

Table 4.7: Comparison of theoretical and experimental mode shapes (FDD)

Table 5.1: Results of regression analyses using period data for RCSW buildings in Montréal (DS-1)

Table 5.2: Results of regression analyses using global period data for SW buildings (DS-2)

Table 5.3: Best-fit, best-fit $-1 \sigma$, and best-fit $+1 \sigma$ equations obtained from regression analyses

Table 5.4: Results of regression analyses for fundamental torsion period of RCSW buildings in Montréal

Table 5.5: Results of regression analyses for second translation period of RCSW buildings in Montréal 160

Table 5.6: Results of regression analyses for second torsion period of RCSW buildings in Montréal 


\section{List of symbols}

All variables in this thesis are expressed in SI units. Unless otherwise stated, default units are $\mathrm{N}, \mathrm{m}, \mathrm{s}$. Variables used in pre-processing of ambient vibration records (section 3.2.1) and regression analyses are defined directly in the text. Below is a list of symbols used in this thesis. These variables and units are defined in the text the first time they are used, and these definitions are repeated for emphasis where it is deemed useful.

\section{Dynamic response of a linear SDOF oscillator}

$a(t) \quad$ Acceleration of an SDOF oscillator

c Viscous damping constant

$c_{c r i t} \quad$ Critical viscous damping constant

$f(t) \quad$ Applied force to an SDOF oscillator

$f_{d}(t) \quad$ Damping force

$f_{e}(t) \quad$ Elastic force

$f_{n} \quad$ Undamped natural frequency of an SDOF oscillator [in $\left.\mathrm{Hz}\right]$

$h(t) \quad$ Impulse response function of an SDOF oscillator

$k \quad$ Stiffness of an SDOF oscillator

$m \quad$ Mass of an SDOF oscillator

$t \quad$ Variable corresponding to time

$T_{n} \quad$ Undamped natural period of an SDOF oscillator [in s]

$x(t) \quad$ Displacement of an SDOF oscillator

$\dot{x}(t) \quad$ Velocity of an SDOF oscillator

$\ddot{x}(t) \quad$ Acceleration of an SDOF oscillator

$\omega_{d} \quad$ Damped natural frequency of an SDOF oscillator [in rad/s]

$\omega_{n} \quad$ Undamped natural frequency of an SDOF oscillator [in rad/s] 
$\xi \quad$ Viscous damping ratio of an SDOF oscillator [expressed as a fraction of critical]

\section{The Fourier transform}

$i \quad$ Imaginary unit $(i=\sqrt{-1})$

$p(t) \quad$ Arbitrary continuous time signal

$P(\omega) \quad$ Fourier transform of continuous time signal $p(t)$ [in units of $p(t) \operatorname{per}(\mathrm{rad} / \mathrm{s})]$

$\theta \quad$ Arbitrary angle [in rad]

$\omega \quad$ Variable corresponding to frequency [in rad/s]

\section{Frequency domain response of a linear SDOF oscillator}

$F(\omega) \quad$ Fourier spectrum obtained from Fourier transform of applied force $f(t)$

$H(\omega) \quad$ Frequency response function (FRF) of an SDOF oscillator

$X(\omega) \quad$ Fourier spectrum obtained from Fourier transform of response $x(t)$

\section{Ground motion and response spectra}

$S_{a}\left(\omega_{n}, \xi\right) \quad$ Pseudo-acceleration spectrum

$S_{d}\left(\omega_{n}, \xi\right) \quad$ Relative displacement spectrum

$S_{v}(\omega, \xi) \quad$ Pseudo-velocity spectrum

$u(t) \quad$ Displacement of an SDOF oscillator relative to the ground

$\dot{u}(t) \quad$ Velocity of an SDOF oscillator relative to the ground

$\ddot{u}(t) \quad$ Acceleration of an SDOF oscillator relative to the ground

$x_{g}(t) \quad$ Ground displacement

$\ddot{x}_{g}(t) \quad$ Ground acceleration

\section{Base shear}

$g \quad$ Acceleration due to gravity $\left(9.81 \mathrm{~m} / \mathrm{s}^{2}\right)$

$V_{\max } \quad$ Maximum base shear of an SDOF oscillator 
$W \quad$ Total building weight

\section{Shear building model for multi-storey buildings}

[C] Damping matrix for a viscously-damped linear MDOF oscillator

$\{F(t)\} \quad$ Vector of forces at discrete dynamic degrees of freedom (DOF)

$[K] \quad$ Stiffness matrix for a linear MDOF oscillator

[M] Mass matrix for a linear MDOF oscillator

$N \quad$ Number of DOF

$\{x(t)\} \quad$ Vector of displacements of the various DOF

$\{\dot{x}(t)\} \quad$ Vector of velocities of the various DOF

$\{\ddot{x}(t)\} \quad$ Vector of accelerations of the various DOF

\section{Dynamic response of a linear MDOF oscillator}

$\left[C^{*}\right] \quad$ Modal (or generalized) damping matrix

$\left[K^{*}\right] \quad$ Modal (or generalized) stiffness matrix

$K_{r} \quad$ Modal stiffness corresponding to mode $r$

[ $\left.M^{*}\right] \quad$ Modal (or generalized) mass matrix

$M_{r} \quad$ Modal mass corresponding to mode $r$

$\{q(t)\} \quad$ Vector of modal (or generalized) coordinates

$\{\dot{q}(t)\} \quad$ First derivative of modal coordinate vector with respect to time

$\{\ddot{q}(t)\} \quad$ Second derivative of modal coordinate vector with respect to time

[Ф] Mode shape matrix

$\left\{\phi_{r}\right\} \quad$ Mode shape $r$

$\omega_{r} \quad$ Natural frequency of mode $r$ [in rad/s]

$\xi_{r} \quad$ Viscous damping ratio of mode $r$ [in fraction of critical]

\section{Frequency domain response of a linear MDOF oscillator}

$F_{k}(\omega) \quad$ Fourier spectrum of input force at DOF $k$ 
$\{F(\omega)\}$ Vector of Fourier spectra of input forces at all DOF (of size $m \times 1$ for each frequency $\omega$ )

$H_{j k}(\omega)$ Frequency response function (FRF) between input at DOF $k$ and output at DOF $j$

$[H(\omega)] \quad$ FRF matrix (of size $N x m$ for each frequency $\omega$ )

$m \quad$ Number of DOF where inputs are applied

$X_{j}(\omega) \quad$ Fourier spectrum of response at DOF $j$

$\{X(\omega)\}$ Vector of Fourier spectra of responses at all DOF (of size $N \times 1$ for each frequency $\omega$ )

$\phi_{j r} \quad$ Element of mode shape $r$ corresponding to DOF $j$

$\phi_{k r} \quad$ Element of mode shape $r$ corresponding to DOF $k$

\section{Response spectrum analysis for a linear MDOF oscillator}

$\left|u_{j}\right| \quad$ Maximum relative displacement of DOF $j$

$\left|u_{j, r}\right| \quad$ Maximum relative displacement of DOF $j$ due to mode $r$

\section{Overview of seismic design provisions in Canada}

$h \quad$ Building height above ground [in $\mathrm{m}]$

$I_{E} \quad$ Earthquake importance factor of a building

$M_{v} \quad$ Higher mode factor

$R_{d} \quad$ Ductility-related force modification factor

$R_{o} \quad$ Overstrength-related force modification factor

$S\left(T_{a}\right) \quad$ Spectral acceleration obtained from UHS at the design fundamental period (also depends on site class)

$T_{a} \quad$ Fundamental period assumed in design [in s]

$T_{1} \quad$ Fundamental period [in s]

$V \quad$ Design base shear 


\section{Dynamic properties of RCSW buildings}

$A_{B} \quad$ Building plan area (or building plan area at base)

$A_{e} \quad$ Equivalent shear area [in $\% A_{B}$ ]

$A_{i} \quad$ Plan area of of shear wall $i$

D Width of all shear walls

$D_{B} \quad$ Dimension of the building at the base in the direction under consideration

$D_{i} \quad$ Width of shear wall $i$

$f_{1} \quad$ Natural frequency of fundamental translation mode [in $\left.\mathrm{Hz}\right]$

$f_{1 t} \quad$ Natural frequency of fundamental torsion mode [in $\left.\mathrm{Hz}\right]$

$f_{j} \quad$ Natural frequency of mode $j[$ in $\mathrm{Hz}]$

$f_{0 j} \quad$ Low-amplitude natural frequency of mode $j$ [in $\left.\mathrm{Hz}\right]$

$h \quad$ Building height above ground [in $\mathrm{m}]$

$h_{i} \quad$ Height of shear wall $i$ above base

$h_{w} \quad$ Maximum height of shear walls above base

I Second moment of area of building in direction under consideration (calculated as sum of second moments of individual walls is same direction)

$L_{w} \quad$ Total wall width per unit plan area $\left[\mathrm{in} \mathrm{m}^{-1}\right]$

$n \quad$ Number of storeys

$N W \quad$ Number of shear walls

$p \quad$ Ratio of wall area to floor plan area for all walls aligned with lateral direction under consideration

$T_{1} \quad$ Fundamental translation period [in s]

$T_{1 t} \quad$ Fundamental torsion period [in s]

$T_{2} \quad$ Natural period of second translation mode [in s]

$T_{2 t} \quad$ Natural period of second torsion mode [in s] 
$x_{h} \quad$ Maximum displacement at the top of the building

$\xi_{1} \quad$ Viscous damping ratio of fundamental translation mode [in fraction of critical]

$\xi_{1 t} \quad$ Viscous damping ratio of fundamental torsion mode [in $\%$ critical]

$\xi_{j} \quad$ Viscous damping ratio of mode $j$ [in fraction of critical]

$\xi_{0 j} \quad$ Low-amplitude viscous damping ratio of mode $j$ [in fraction of critical]

$\xi_{1 j} \quad$ Rate of change of damping ratio of mode $j$ with vibration amplitude [in fraction of critical, see equation 2.51]

\section{Experimental modal analysis}

$F_{k}(\omega) \quad$ Fourier spectrum of input force at DOF $k$

$G_{F k X j}(\omega)$ Spectral density between input at DOF $k$ and output at DOF $j$ (similarly for spectral densities between other signals)

$H_{j k}(\omega)$ Frequency fesponse function (FRF) between input at DOF $k$ and output at DOF $j$

$X_{j}(\omega) \quad$ Fourier spectrum of response at DOF $j$

\section{Stochastic processes}

$N \quad$ Number of sample records of process $x(t)$

$R_{x x}(\tau) \quad$ Autocorrelation function of process $x(t)$

$x(t) \quad$ General stochastic process (or a time history record)

$x_{k}(t) \quad$ Sample record corresponding to an observation of process $x(t)$

$\mu_{x} \quad$ Mean value of process $x(t)$

\section{Spectral density estimation}

$G_{x y}(\omega)$ Spectral density between records $x(t)$ and $y(t)$ 
$m \quad$ Index corresponding to a particular sub-record of $x(t)$ or $y(t)$

$r \quad$ Number of sub-records of $x(t)$ and $y(t)$ used to estimate spectral density

$R_{x y}(\tau) \quad$ Cross-correlation function between records $x(t)$ and $y(t)$

$x(t) \quad$ Arbitrary time history record (or signal)

$X(\omega) \quad$ Fourier spectrum of $x(t)$

$x^{m}(t) \quad$ Sub-record of $x(t)$ of shorter duration

$X^{m}(\omega) \quad$ Fourier spectrum of $x(t)$ estimated from sub-record $x^{m}(t)$

$\tilde{x}^{m}(t) \quad$ Sub-record of $x(t)$ modified by leakage reduction window

$\widetilde{X}^{m}(\omega)$ Fourier spectrum of $x(t)$ estimated from modified sub-record $\tilde{x}^{m}(t)$

$y(t) \quad$ Arbitrarty time history record (or signal)

$Y(\omega) \quad$ Fourier spectrum of signal $y(t)$

$y^{m}(t) \quad$ Sub-record of $y(t)$ of shorter duration

$Y^{m}(\omega) \quad$ Fourier spectrum of $y(t)$ estimated from sub-record $y^{m}(t)$

$\tilde{y}^{m}(t) \quad$ Sub-record of $y(t)$ modified by leakage reduction window

$\widetilde{Y}^{m}(\omega)$ Fourier spectrum of $y(t)$ estimated from modified sub-record $\tilde{y}^{m}(t)$

\section{Experimental methods}

All variables for data pre-processing are defined directly in the text.

$[G(\omega)]$ Output spectral density matrix (also denoted $\left[G_{x x}(\omega)\right]$ for emphasis)

$\left[G_{f f}(\omega)\right] \quad$ Input spectral density matrix

$[H(\omega)] \quad$ FRF matrix

$G_{j k}(\omega) \quad$ Spectral density between records $x_{j}(t)$ and $x_{k}(t)$ 
$G_{j k}{ }^{m}(\omega) \quad$ Spectral density between records $x_{j}(t)$ and $x_{k}(t)$ for subrecord $m$

MAC Modal assurance criterion

$q \quad$ Index corresponding to measurement setup

$R_{v v}(\tau) \quad$ Autospectral density of a linear SDOF oscillator subjected to white noise excitation

$[S] \quad$ Singular value matrix (diagonal)

$s_{i}(\omega) \quad i^{\text {th }}$ singular value at frequency $\omega$

$s_{i}(\omega, q) \quad i^{\text {th }}$ singular value at frequency $\omega$ for measurement setup $q$

$T_{d} \quad$ Damped natural period of an identified resonant frequency

$[U] \quad$ Left-singular vector matrix

$\left\{u_{i}(\omega)\right\} \quad i^{\text {th }}$ singular vector at frequency $\omega$

$\left\{u_{i}(\omega, q)\right\} i^{\text {th }}$ singular vector at frequency $\omega$ for measurement setup $q$

$[V] \quad$ Right-singular vector matrix

$x_{i}(t) \quad$ Velocity time history record corresponding to DOF $i$

$x_{i}^{m}(t) \quad$ Sub-record of $x_{i}(t)$ of shorter duration

$\tilde{x}_{i}^{m}(t) \quad$ Sub-record of $x_{i}(t)$ modified by leakage reduction window

$\delta \quad$ Logarithmic decrement

$\left\{\phi\left(\omega_{p}\right)\right\} \quad$ Mode shape estimate corresponding to frequency $\omega_{p}$

$\omega_{b} \quad$ Frequency included in the SDOF bell of a resonant frequency

$\omega_{p} \quad$ Candidate resonant frequency (peak in singular value plot)

\section{Mathematical operators}

$E[\cdot] \quad$ Expected value

$\exp (x) \quad$ Exponential function $\left(\exp (x)=e^{x}\right)$ 
$\ln (\cdot) \quad$ Natural logarithm

$(\cdot)^{*} \quad$ Complex conjugate

|. $\quad$ Absolute value of a real number, magnitude of a complex number, or norm of a vector

$[\cdot]^{T} \quad$ Transpose of a matrix (or vector)

$[\cdot]^{H} \quad$ Hermitian transformation (complex conjugate transpose)

$\sum \quad$ Summation

$\int \quad$ Integral

\section{Acronyms}

DAS Data acquisition system

DFT Discrete Fourier transform

DOF Degrees of freedom (singular or plural)

EFDD Enhanced frequency domain decomposition

ESFP Equivalent static force procedure

FDD Frequency domain decomposition

FFT Fast Fourier transform

FRF Frequency response function

IRF Impulse response function

LLRS Lateral load-resisting system

MAC Modal assurance criterion

MDOF Multiple-degree-of-freedom

NBCC National Building Code of Canada

PSD Power spectral density

RC Reinforced concrete

RCS Remote control starter

RCSW Reinforced concrete shear wall

SDOF Single-degree-of-freedom

UHS Uniform hazard spectrum 


\section{Introduction}

\subsection{Research motivation}

The behaviour of a structure subjected to dynamic loads, such as strong winds or earthquake ground motions, is a complex phenomenon that depends on the nature of the forces exciting the structure and the dynamic properties of the structure itself, namely its natural periods, mode shapes, and damping characteristics, as well as the evolution of these properties in time if the structure exhibits nonlinear response. Therefore, to simulate the response of a structure to various dynamic loading conditions, engineers must first estimate the structure's dynamic properties in its operational, undamaged state. However, this poses a considerable challenge in structural design as these properties cannot be determined accurately before the structure is constructed. As a result, the assumed properties may be significantly different from those of the actual structure, once it is constructed, due to differences between the idealized structure and in situ conditions.

Typically, these dynamic properties are estimated using either empirical relationships or analytical methods, such as eigenvalue analysis. For seismic design, building codes provide simple empirical equations based on building geometry and applicable to specific construction materials and types of horizontal load-resisting systems, to estimate the fundamental period of a building. The equations currently suggested in several codes were developed based on the measured fundamental periods of buildings in California. However, these equations do not fit these data very well as they do not capture the actual distribution of lateral stiffness and mass within a building, the two parameters that determine its fundamental period. Further, as we will see, linear dynamic building response is often 
simulated assuming constant viscous damping in all low frequency vibration modes, since the exact mechanisms of energy dissipation in vibrating structures are not fully understood (PEER/ATC, 2010). However measured damping values in buildings vary widely and it may not be appropriate to assume the same amount of damping in all vibration modes and in all buildings. Moreover, gross overestimation of damping at the design stage would lead to lower predictions of displacements, accelerations, and stresses than can be realistically expected. Finally, little guidance is provided, in codes or elsewhere, to help engineers validate the values of the natural periods of torsion and higher translation modes used for design purposes.

Clearly, more reliable data on the dynamic properties of real buildings are required to help engineers ensure that the values of these properties used in design are realistic and to craft appropriate recommendations in building codes. Ultimately, this should lead to improved simulation of the behaviour of structures subjected to dynamic loads, particularly in the context of structural design. This was the main motivation behind this research project.

An additional motivating factor for this research was that a few studies have suggested that local design and construction practices, as well as seismicity, may cause regional variations in the dynamic properties of similar buildings (Goel and Chopra, 1998; Hong and Hwang, 2000). Since the formulae adopted in the 2005 National Building Code of Canada to estimate the fundamental period of various types of buildings were developed based on the measured fundamental periods of buildings in California, it is unclear whether they accurately reflect the fundamental periods of buildings in Eastern Canada. 


\subsection{Research objectives and methodology}

The main objectives of this research project are to examine how the models and assumptions recommended in structural codes and technical publications to estimate the dynamic properties of buildings compare to those measured in real buildings, and to determine whether different models can be developed to better represent the dynamic properties of Canadian buildings.

To address these issues, ambient vibration measurements were performed in 39 multi-storey buildings, ranging in height from 12 to $195 \mathrm{~m}$, on the island of Montréal, with the intent of identifying their dynamic properties from the recorded motions. The dynamic properties of up to six vibration modes (the two lowest-frequency translation modes in each principal direction and two lowest-frequency torsion modes) were successfully identified using the enhanced frequency domain decomposition (EFDD) method and the detailed results are presented in a separate published report (Gilles, 2010). Though the initial goal was to obtain a representative sample of different types of structural systems used in Montréal buildings, 27 of the 39 buildings studied turned out to be reinforced concrete buildings with shear walls providing the main resistance to lateral loads. It was thus decided to limit the scope of this thesis to these types of buildings. The identified dynamic properties of the remaining buildings ( 7 concrete moment-resisting frame, 3 steel momentresisting frame, and 3 steel braced frame buildings) are nevertheless available for future studies.

This thesis therefore focuses on the prediction of the following dynamic properties of reinforced concrete shear wall (RCSW) buildings:

- Fundamental translation period, 
- Damping, and

- Natural periods of torsion and second translation modes.

Note that each natural period is the reciprocal of the corresponding natural frequency. The measured dynamic properties of the 27 RCSW buildings studied are compared to various models proposed in the literature. Indeed, with regards to the fundamental translation periods, the current equation proposed in several codes (ASCE, 2005; NRC/IRC, 2005a; SEAOC, 1999), as well as the models suggested by Goel and Chopra (1998), Lee et al (2000), Morales (2000), and Wallace and Moehle (1992) are considered. To evaluate common assumptions regarding damping in buildings, the recommendations of the NBCC 2005 and the models proposed by Jeary (1986), Lagomarsino (1993), and Satake et al (2003) are examined. Since no recommendations are currently available in codes, the natural periods of torsion and second translation modes are compared to the models proposed by Lagomarsino (1993) and Satake et al (2003). Finally, regression analyses are performed to see if better fits to the measured data can be obtained using different models to estimate these dynamic properties.

\subsection{Scope of this study}

Based on regression analyses, simple models are proposed to estimate the mean values of the natural periods of the first two (i.e. having the lowest frequency, or the longest period) translation modes in each direction, as well as the first two torsion modes, of RCSW buildings. To provide a measure of the uncertainty associated with these predictions, equations corresponding to the mean minus one standard deviation and mean plus one standard deviation are also presented. These results show that the equation adopted in the 2005 NBCC to estimate the fundamental period of RCSW buildings can be improved. Further, the results for the 
other modes of vibration provide a realistic range of values for their respective natural periods. These could be an appropriate starting point for code recommendations on torsion and higher vibration modes.

With regards to damping, the measured damping values are shown to vary widely for the different buildings studied and none of the proposed models considerably reduce this variability. Based on these measured damping values, recommendations are made regarding conservative damping values to use for the design of RCSW buildings.

Note that the results presented herein are limited to RCSW buildings. Before these recommendations are considered for adoption in building codes, it would be useful to conduct similar studies on buildings made of different materials, and comprising different lateral load-resisting systems. Since all the buildings studied were located in Montréal, which is itself located in a zone of moderate seismic activity representative of the tectonic setting of Eastern Canada, similar studies should also be performed in other urban centres (such as Toronto, with low seismicity, and Vancouver, with high seismicity) to verify whether the dynamic properties of this type of building are consistent across Canada.

Furthermore, the dynamic properties of a building are likely affected by the foundation and soil conditions at the site (Ghrib and Mamedov, 2004). In future, it may be possible to account for soil-structure interaction in the prediction of the dynamic properties of building systems; however, widely accepted methods to achieve this are not currently available. For this reason, these effects were not considered in this study.

Finally, another important limitation of this study is that the dynamic properties of the 27 RCSW buildings were identified from ambient vibration records, i.e. under normal operating conditions. Several studies 
have shown that the natural periods of a building system can elongate during strong ground shaking, and damping can increase as well. These phenomena are likely due to damage to structural and non-structural components and nonlinear soil behaviour (Clinton et al., 2006; Trifunac et al., 2001b). However, no convincing relationships have been proposed to quantify the elongation of the natural periods, nor the increase in damping, that can be expected from ambient to strong ground motions. Strictly speaking, the models derived from this data set therefore represent the dynamic properties of RCSW buildings at low vibration amplitudes and are deemed to represent the initial conditions of buildings subjected to strong ground shaking.

Despite its limitations, this study nevertheless provides important and useful data on the dynamic properties of real RCSW buildings in Eastern Canada. In future, the development of methods to account for soilstructure interaction, amplitude effects, and regional variations should allow more accurate determination of the dynamic properties of buildings for design purposes.

Finally, note that the 2005 edition of the National Building Code of Canada specifies that seismic loads should, by default, be determined using dynamic analysis methods, while equivalent static methods can still be used to determine wind loads in most cases, except in very tall structures. For this reason, the focus of this thesis is mainly on the estimation of dynamic properties for seismic analysis and design. However, much of the discussion can be extended to the dynamic effects of wind.

\subsection{Organization of thesis}

Chapter 2 begins with a summary of structural dynamics concepts, which is intended primarily for the non-expert reader to gain a general 
understanding of the dynamic behaviour of buildings. This is followed by a brief overview of the seismic design provisions in the 2005 edition of the National Building Code of Canada, with particular emphasis on the methods that can be used to determine design seismic loads and predict the dynamic properties of buildings. Then, a review of relevant literature on the dynamic properties of RCSW buildings, experimental modal analysis, ambient vibration tests, and stochastic processes is presented.

Chapter 3 discusses in detail the methods used in this study to collect and analyze ambient vibration data to identify the dynamic properties of the various buildings. First, the relevant technical specifications of the testing equipment are discussed and the testing procedure is described. Particular attention is given to the issue of synchronization of recorded signals, since two independent data acquisition systems were used in this study. The theory behind the enhanced frequency domain decomposition (EFDD) method, which was used in this study to extract the dynamic properties from the recorded ambient motions, is then summarized. To illustrate how the method is implemented, Appendix B shows the identification of the first two (lowest-frequency) vibration modes of one of the buildings studied.

To ensure that the dynamic properties identified from ambient tests were consistent and reliable, Chapter 4 presents a validation of the data analysis methods. First, a simple numerical model having six dynamic degrees of freedom (DOF) was created and its "theoretical" natural frequencies and mode shapes were determined from eigenvalue analysis. Constant viscous damping was assumed for all vibration modes. The model structure was then subjected to artificial dynamic loads and the responses of various points were extracted, simulating the procedure used in this study to measure ambient motions in real buildings. These simulated responses were then used with EFDD to identify the 
"experimental" dynamic properties of the model structure. The theoretical and experimental dynamic properties are shown to be nearly identical, though there are slight discrepancies, particularly for damping in higher vibration modes. Further, ambient vibration tests were performed in certain buildings on separate occasions. The dynamic properties identified on different days for each of these buildings are shown to be consistent.

Chapter 5 shows how the dynamic properties of the 27 RCSW buildings in this study compare to the various models that have been proposed in buildings codes and in the literature. Regression analyses are described and the results are used to develop improved models to predict the natural periods of the first six (lowest-frequency) vibration modes of RCSW buildings. Further, to provide a measure of the uncertainty associated with each of these predictive models, the equations corresponding to the bestfit, best-fit minus one standard deviation, and best-fit plus one standard deviation are presented.

Finally, Chapter 6 summarizes the main conclusions of this research and its original contributions to structural dynamics and earthquake engineering. These are followed by a reiteration of the limitations of this study and recommendations on future work. 


\section{Background and literature review}

In section 2.1, some of the more important structural dynamics concepts, as pertains to this study, are briefly summarized. It is intended primarily for the non-expert reader to gain an overall understanding of the behaviour of structures subjected to dynamic loads. This is followed in section 2.2 by an overview of the seismic design provisions of the 2005 edition of the National Building Code of Canada (NBCC). Particularly, the way in which seismic hazard is specified, the analysis methods that can be used to determine design seismic loads, and the recommendations regarding the estimation of dynamic building properties are highlighted. The following sub-sections present a review of the literature on the dynamic properties of reinforced concrete shear wall (RCSW) buildings (section 2.3), experimental modal analysis with particular emphasis on the extraction of dynamic properties from ambient vibration tests (section 2.4), and stochastic processes (section 2.5). Note that much of this material has been adapted from a published report (Gilles, 2008).

\subsection{Structural dynamics concepts}

The response of a building to dynamic loads, such as strong winds or earthquake ground motions, is a complex, dynamic phenomenon that depends on the characteristics of the excitation and the dynamic properties of the building system. To understand what the various dynamic properties of a building represent, and to appreciate their importance in dynamic building response, let us first review some fundamental structural dynamics concepts. Much of the following is standard textbook material, summarized mainly from Craig and Kurdila (2006). Other excellent references include Chopra (2001), Clough and Penzien (2003), and Humar (2002). 


\subsubsection{Dynamic response of a linear SDOF oscillator}

The lateral motion of a single-storey building, in a given direction, can be modeled as a linear SDOF oscillator, having its mass $(m)$ lumped at the roof level, and elastic forces provided by a spring of equivalent stiffness $(k)$ representing the combined lateral stiffness of vertical structural elements, such as columns, braces, and walls. This assumes that the roof is rigid in its own plane (i.e. the entire roof moves horizontally as a rigid body), and that the vertical elements are flexible to lateral deformation, but rigid vertically. Further, the various mechanisms of energy dissipation can be represented by an equivalent damper (or dashpot). If a lateral force is applied to the mass, a simple equilibrium of forces at any instant in time shows that

$$
f(t)-f_{e}(t)-f_{d}(t)=m a(t)
$$

where $f(t)$ is the applied force, $f_{e}(t)$ is the elastic force in the equivalent spring, $f_{d}(t)$ is the damping force and $a(t)$ is the acceleration of the mass, at time $t$, which defines the inertia force. The elastic force is proportional to the displacement of the mass relative to its equilibrium position (or the elongation of the spring), which is defined as $x(t)$. In contrast, the damping force is more difficult to evaluate as the mechanisms of damping in structural systems are varied and complex. It is most common to use a viscous damping model, in which the damping force is proportional to the velocity of the mass, to represent the damping force. It should be noted that this model is selected based on mathematical convenience in the time domain analysis, rather than on a rigorous understanding of the underlying mechanisms. 
Equation 2.1 can then be rewritten to yield the equation of motion of a linear SDOF oscillator subjected to a generalized force, as follows:

$$
m \ddot{x}(t)+c \dot{x}(t)+k x(t)=f(t)
$$

where $x(t)$ represents displacement, $\dot{x}(t)$ represents velocity, $\ddot{x}(t)$ represents acceleration, and $c$ represents the viscous damping constant. This is the classical second order differential equation with constant coefficients.

We define the undamped natural (circular) frequency (in rad/s) of the oscillator as

$$
\omega_{n}=\sqrt{\frac{k}{m}}
$$

which is the frequency at which the SDOF system would oscillate if displaced from its equilibrium position (and/or imparted with an initial velocity) and allowed to oscillate freely, in the absence of any external force and damping force $\left(f(t)=f_{d}(t)=0\right)$. Alternatively, we can define the oscillation of the SDOF system in terms of its natural frequency (in cycles/s or $\mathrm{Hz}$ )

$$
f_{n}=\frac{\omega_{n}}{2 \pi}
$$

or its natural period 


$$
T_{n}=\frac{1}{f_{n}}=\frac{2 \pi}{\omega_{n}}
$$

Further, it is common to specify damping relative to the critical viscous damping value, which represents the minimum amount of damping for which the free response of the SDOF system would not involve any oscillation about the equilibrium position, as follows:

$$
\xi=\frac{c}{c_{c r i t}}=\frac{c}{2 m \omega_{n}}
$$

where $c_{c r i t}$ is the critical damping value, and $\xi$ is the viscous damping ratio. Finally, we define the damped natural (circular) frequency as

$$
\omega_{d}=\omega_{n} \sqrt{1-\xi^{2}}
$$

By considering the applied force as a series of impulses occurring at time $\tau$, the forced displacement response with zero initial conditions can be obtained in the time domain using the convolution (or Duhamel) integral,

$$
x(t)=\int_{0}^{t} f(\tau) h(t-\tau) d \tau
$$

in which $h(t)$ represents the unit impulse response function (IRF) of the oscillator, which is given by

$$
h(t)=\frac{1}{m \omega_{d}} \exp \left(-\xi \omega_{n} t\right) \sin \left(\omega_{d} t\right)
$$


Note that the IRF is a characteristic of the oscillator, which involves both its natural frequency and its damping ratio, and represents the SDOF response to a unit impulse force applied at time $t=0$. The other response quantities (velocity and acceleration) can be obtained by differentiating equation 2.8 .

\subsubsection{The Fourier transform}

As we will see in section 2.1.3, the response can alternatively be obtained in the frequency domain by making use of the Fourier transform of the input force and the frequency response function (FRF) of the system. The Fourier transform of a continuous time signal, $p(t)$, is defined as

$$
P(\omega)=\int_{-\infty}^{\infty} p(t) \exp (-i \omega t) d t
$$

In the above equation, a complex exponential representation of harmonic functions is adopted, in which

$$
\exp (i \theta)=\cos \theta+i \sin \theta
$$

The Fourier transform function, $P(\omega)$, is a continuous, complex function that provides a frequency domain representation of the time signal, $p(t)$. Therefore, the Fourier transform is very useful in identifying the frequency content of a particular time signal.

Conversely, the original time signal can be recovered from its frequency domain representation using the inverse Fourier transform: 


$$
p(t)=\frac{1}{2 \pi} \int_{-\infty}^{\infty} P(\omega) \exp (i \omega t) d \omega
$$

Hence, the functions $p(t)$ and $P(\omega)$ are said to form a Fourier pair.

However, in engineering applications, the function $p(t)$ is often sampled at discrete time intervals for a finite period of time, leading to a finite-length, digital (non-continuous) representation of the time signal. In such instances, the Fourier integral transforms of equations 2.10 and 2.12 are not directly applicable; rather, a digital form of these equations, known as the discrete Fourier transform (DFT), must be used. Typically, the DFT operation is performed using the fast Fourier transform (FFT) algorithm, developed by Cooley and Tukey (1965). The result of this operation is a discrete (rather than continuous), complex, frequency domain representation of the original discrete signal. Note that the duration of the sampled data record (for instance in seconds) used to compute the FFT dictates the frequency spacing of the frequency domain representation, while the number of sampled data points dictates the frequency range. For example, a function sampled at $100 \mathrm{~Hz}$ for $50 \mathrm{~s}$ would involve 5000 data points. Taking the FFT of this data record would lead to a frequency spacing of $0.02 \mathrm{~Hz}(\Delta f=1 / 50 s)$ and a frequency range of $0-50 \mathrm{~Hz}$ $\left(f_{\max }=(5000 / 2) \Delta f\right)$.

\subsubsection{Frequency domain response of a linear SDOF oscillator}

Instead of convolving the IRF and the input force in the time domain, the response of an SDOF system can be obtained in the frequency domain by simply multiplying the Fourier transform of the forcing function, $F(\omega)$, and the complex frequency response function (FRF), $H(\omega)$, of the oscillator: 


$$
X(\omega)=H(\omega) F(\omega)
$$

in which the FRF is given by

$$
H(\omega)=\frac{1}{k\left(\left[1-\left(\omega / \omega_{n}\right)^{2}\right]+i\left[2 \xi\left(\omega / \omega_{n}\right)\right]\right)}
$$

From Equation 2.13, it is clear that the FRF acts as a filter (or transfer function) between the input $F(\omega)$ and the response $X(\omega)$. If the input force has significant energy near the oscillator's natural frequency, a resonance phenomenon will ensue, in which the response will be significantly amplified. Similarly to the IRF, the FRF contains all the information about the system's dynamic properties and represents the forced response (with zero initial conditions) to a unit harmonic excitation. The IRF and FRF are Fourier pairs: it follows that the convolution of any time function $f(t)$ with the IRF, as in equation 2.8 , is equivalent to the product in the frequency domain of its Fourier transform $F(\omega)$ and the FRF, as in equation 2.13.

Moreover, a plot of the magnitude of the FRF against frequency will exhibit a distinct peak at the natural frequency of the oscillator, and the sharpness of this peak is determined by the amount of damping, with lightly damped oscillators displaying sharper peaks.

\subsubsection{Ground motion and response spectra}

In many practical structural dynamics applications, the temporal variation of building response - for instance due to wind or earthquake effects - is of little importance; rather, structural engineers are mainly concerned with the maximum response. A response spectrum is a plot of the maximum 
response of all SDOF oscillators, having a given level of damping, to a specific input force. The abscissa is usually the natural frequency or the natural period of the SDOF oscillator, sometimes represented in the logarithmic scale, while the ordinate is the maximum magnitude of a specific response indicator observed during or after the application of the input force. In earthquake engineering, three important response spectra are widely used: the relative displacement $\left(S_{d}\right)$, pseudo-velocity $\left(S_{v}\right)$, and pseudo-acceleration $\left(S_{a}\right)$ spectra.

The earthquake ground motion problem is one of relative motion of the mass with respect to its support: the inertia force depends on the absolute acceleration of the mass, whereas the elastic and damping forces depend on relative displacement and velocity, respectively. In the absence of a force $f(t)$ applied directly at the mass, the dynamic equilibrium of equation 2.2 is rewritten as

$$
m \ddot{u}(t)+c \dot{u}(t)+k u(t)=-m \ddot{x}_{g}(t)
$$

In the above equation, $\ddot{x}_{g}$ represents the ground acceleration, and $u$ represents the displacement of the mass relative to the ground and is given by

$$
u(t)=x(t)-x_{g}(t)
$$

The relative displacement of an SDOF oscillator of natural frequency $\omega_{n}$, and damping ratio $\xi$, subjected to a given ground motion, can then be obtained using the convolution integral of equation 2.8 by replacing the forcing function by the appropriate inertial force on the right-hand side of equation 2.15. In most cases, a numerical solution is used, from which the 
maximum relative displacement is identified. This procedure is repeated for different natural frequencies to obtain the displacement spectrum, $S_{d}$.

The pseudo-velocity and pseudo-acceleration spectra are then defined from the displacement spectrum, as follows:

$$
\begin{aligned}
& S_{v}\left(\omega_{n}, \xi\right)=\omega_{n} S_{d}\left(\omega_{n}, \xi\right) \\
& S_{a}\left(\omega_{n}, \xi\right)=\omega_{n}^{2} S_{d}\left(\omega_{n}, \xi\right)
\end{aligned}
$$

The prefix pseudo refers to the fact that, unlike the displacement response spectrum, these spectra do not truly represent the maximum relative velocity and absolute acceleration of a linear viscously-damped SDOF oscillator. They are approximations to the true relative velocity and absolute acceleration response. However, since most building structures are only lightly damped (typically less than 5 percent critical for an undamaged building not having any special damping devices), these approximations (particularly pseudo-acceleration) are quite accurate. Further, the difference between the undamped and damped natural frequency is typically ignored. Once these spectra have been developed for a particular base excitation, they can be used to predict the maximum response of any linear SDOF system to that same excitation.

\subsubsection{Base shear}

Referring back to equations 2.1 and 2.2, the elastic force in the equivalent spring can be obtained from the displacement response. For the base motion problem presented above, the maximum force in the equivalent spring is thus obtained from either the spectral displacement or the pseudo-acceleration, as follows: 


$$
\left(f_{e}\right)_{\text {max }}=k|u|_{\text {max }}=k S_{d}\left(\omega_{n}, \xi\right)=m S_{a}\left(\omega_{n}, \xi\right)
$$

This illustrates the importance of the pseudo-acceleration response spectrum in earthquake engineering: once it is known, only the mass, natural frequency, and viscous damping ratio of the SDOF oscillator are required to estimate the maximum elastic force that it will experience during a particular ground motion. In a building subjected to base motion, the elastic force represents the total force that must be resisted by all the elements providing lateral stiffness (columns, frames, braces, and walls). Indeed, each of these elements, if present, will attract a fraction of the total elastic force in proportion to its individual lateral stiffness. Since it corresponds to the horizontal reaction at the base of the building, the total horizontal elastic force is referred to as base shear, and denoted $V$. The base shear forms the basis of the lateral seismic force requirements in most building codes, and is usually calculated using a slightly different equation:

$$
V_{\max }=\frac{S_{a}\left(\omega_{n}, \xi\right)}{g} W
$$

in which the reactive mass of the building is replaced by $W$, the total building weight, divided by $g$, the acceleration due to gravity.

\subsubsection{Shear building model for multi-storey buildings}

In multi-storey buildings, it is not appropriate to assume that the entire building mass, stiffness, and damping can be lumped at a single location. Further, to simulate the response of a building to dynamic loads, it is often necessary to consider its motion in more than a single direction. For these reasons, multi-storey buildings are typically modeled as multiple-degree- 
of-freedom (MDOF) systems, in which the mass, stiffness, and damping are not concentrated at a single location, but distributed along the height and plan of the building at discrete points. It is common to use a lumped parameter approach, whereby mass, stiffness, and damping are lumped at each floor level, typically at its centre of rigidity. In Cartesian coordinates, the complete motion of any point in the structure can be described by translations in three orthogonal directions, and rotations about three orthogonal axes. In buildings subjected to wind or earthquakes, tilting motions are typically small and the rotatory inertia terms associated with rotations about the two horizontal axes are neglected, and these two dynamic degrees of freedom (DOF) are eliminated. Further, by assuming that each floor acts as a rigid diaphragm and that the vertical elements are rigid vertically, the motion of each floor level can be described by three dynamic DOF: translations in two horizontal directions (in the plane of the floor) and in-plane rotation about the vertical axis of the building (or torsion). This is the model most commonly used to represent the lateral motions of multi-storey buildings and is commonly referred to as the shear building model.

The equations of motion for a linear MDOF, viscously-damped, elastic, shear building model is

$$
[M]\{\ddot{x}(t)\}+[C]\{\dot{x}(t)\}+[K]\{x(t)\}=\{F(t)\}
$$

On the left-hand side of equation 2.21, the variables in square brackets are the coefficient matrices containing mass/inertia $(M)$, damping $(C)$, and stiffness $(K)$ terms corresponding to each dynamic DOF defined, and the variables in curly brackets are vectors representing the acceleration, velocity, and displacement of the various DOF. For the shear building model described above, these matrices and vectors may have entries corresponding to different directions of motion for each floor - two 
translations and one rotation - so the system of equations is of dimension $N=3 n$, where $n$ is the total number of floors above ground.

\subsubsection{Dynamic response of a linear MDOF oscillator}

In general, the coefficient matrices in equation 2.21 contain non-zero offdiagonal terms, or coupling terms. Therefore, to solve it would involve simultaneously solving $N$ equations in $N$ unknowns. The normal mode superposition method is a technique used in lightly-damped systems to reduce these coupled linear equations into a set of uncoupled linear equations by making use of the undamped natural modes of the structure to define a new coordinate system (Craig and Kurdila, 2006).

The first step of the mode superposition method is to find the undamped natural modes of the system by solving an eigenvalue problem, using any of the numerical procedures described in most structural dynamics textbooks. For an $N$-DOF system, there are $N$ modes of vibration (eigenvectors), $\left\{\phi_{r}\right\}, r=1,2, \ldots, N$, each having a corresponding undamped natural frequency (related to the eigenvalues), $\omega_{n, r}$. and which can be combined to form an $N \times N$ modal matrix, [Ф], as follows:

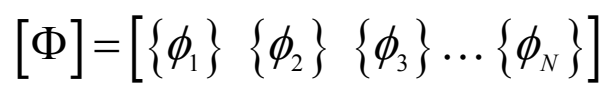

Using a separation of variables, along with the expansion theorem, the displacement vector can be represented by a linear combination of spatially-varying components (the mode shapes), and time-varying components, referred to as the modal (or generalized) coordinates:

$$
\{x(t)\}=[\Phi]\{q(t)\}
$$


which allows the equations of motion (2.21) to be recast as

$$
\left[M^{*}\right]\{\ddot{q}(t)\}+\left[C^{*}\right]\{\dot{q}(t)\}+\left[K^{*}\right]\{q(t)\}=[\Phi]^{T}\{F(t)\}
$$

in which

$$
\begin{aligned}
& {\left[M^{*}\right]=[\Phi]^{T}[M][\Phi]=\operatorname{diag}\left[M_{r}\right]} \\
& {\left[K^{*}\right]=[\Phi]^{T}[K][\Phi]=\operatorname{diag}\left[\omega_{r}{ }^{2} M_{r}\right]}
\end{aligned}
$$

Because of the orthogonality properties of normal mode shapes (Craig and Kurdila, 2006), the modal (or generalized) mass matrix, $\left[M^{*}\right]$, is diagonal with values of $M_{r}$ corresponding to the $r^{\text {th }}$ mode. The modal mass corresponding to mode $r$ is given by

$$
M_{r}=\left\{\phi_{r}\right\}^{T}[M]\left\{\phi_{r}\right\}
$$

Similarly, the modal stiffness matrix, $\left[K^{*}\right]$, is diagonal with values of $\omega_{r}^{2} M_{r}$ corresponding to the $r^{\text {th }}$ mode. On the other hand, the viscous damping matrix, $[C]$, would generally not be diagonalized by the modal matrix. It is therefore common to specify an overall viscous damping ratio for each vibration mode, $\xi_{r}$, rather than localized damping coefficients. This assumes that the modal matrix diagonalizes the damping matrix, as follows:

$$
\left[C^{*}\right]=[\Phi]^{T}[C][\Phi]^{\text {Assumed }}=\operatorname{diag}\left[2 \xi_{r} \omega_{r} M_{r}\right]
$$


Under the above assumptions, the equations of motion are fully uncoupled, and can thus be solved independently for each mode. For mode $r$,

$$
M_{r} \ddot{q}_{r}(t)+2 \xi_{r} \omega_{r} M_{r} \dot{q}_{r}(t)+\omega_{r}{ }^{2} M_{r} q_{r}(t)=\left\{\phi_{r}\right\}^{T}\{F(t)\}
$$

Similarly to equation 2.2 , this equation can be solved using the convolution integral, making use of the impulse response function for each mode. The modal coordinates for each mode, $q_{r}(t)$, represent the contribution of each vibration mode to the overall motion of the structure, resulting from the applied forces. These individual contributions can then be superimposed to recover the total motion of the structure:

$$
\{x(t)\}=[\Phi]\{q(t)\}=\sum_{r=1}^{N}\left\{\phi_{r}\right\} q_{r}(t)
$$

Another very common approach is to assume the damping matrix to be a linear combination of the stiffness and mass matrices, which also leads to a set of uncoupled linear equations. Note that, in either case, the response can be adequately simulated by considering the contributions of only a limited number of vibration modes, which are more excited than others by their generalized load function (right-hand side of equation 2.29). Hence, it is common to use a truncated modal superposition approach in seismic analysis, since the high-energy frequency content of earthquakes typically excites only the lower-frequency modes of buildings (below $10 \mathrm{~Hz}$ ).

\subsubsection{Frequency domain response of a linear MDOF oscillator}

Similarly to linear SDOF systems, the input at a particular DOF can be related to the output at another DOF by a frequency response function 
(FRF), which contains the dynamic properties of the system. But clearly, for MDOF systems, a given input at a particular DOF will not cause the same output at all DOF, and the output at a particular DOF will not be the same if the same input is applied at different DOF. Therefore, a different FRF exists between each input and each output. For an $N$-DOF system with inputs at $m$ DOF, the different FRF can be represented by an $N \times m$ FRF matrix at each frequency $\omega$. Thus, element $H_{j k}(\omega)$ of the FRF matrix represents the relationship between the output at DOF $j, X_{j}(\omega)$, and the input at DOF $k, F_{k}(\omega)$,

$$
X_{j}(\omega)=H_{j k}(\omega) F_{k}(\omega)
$$

and is given by

$$
H_{j k}(\omega)=\sum_{r=1}^{N} \frac{\phi_{j r} \phi_{k r}}{K_{r}} \frac{1}{\left[1-\left(\omega / \omega_{r}\right)^{2}\right]+i\left[2 \xi_{r}\left(\omega / \omega_{r}\right)\right]}
$$

in which $K_{r}$ is the modal stiffness for mode $r, \phi_{j r}$ is the element of the $r^{\text {th }}$ mode corresponding to DOF $j$, and $\phi_{k r}$ is the element of the $r^{\text {th }}$ mode corresponding to DOF $k$.

Similarly to equation 2.13 for SDOF systems, instead of using the convolution integral, the response can be computed in the frequency domain using

$$
\{X(\omega)\}=\underset{N x 1}{[H(\omega)}]\{\underset{m x 1}{H}(\omega)\}
$$

in which the underscripts represent the size of the vectors and matrix. Again, the FRF matrix acts as a filter (or transfer function) between the 
inputs and the responses, contains all the information about the system's dynamic properties, and is independent of the input forces. Moreover, a plot of the magnitude of any FRF against frequency will exhibit distinct peaks at the natural frequencies of the oscillator's various modes of vibration, and the sharpness of each peak is determined by the amount of damping in the corresponding mode.

\subsubsection{Response spectrum analysis for a linear MDOF oscillator}

To estimate the maximum response of a linear MDOF system to ground motions, response spectrum analysis is commonly used. Since each vibration mode has its own natural frequency, the maximum response due to each mode can be obtained from the appropriate response spectrum (with minor algebraic manipulations). The maximum response must then be calculated from the maximum responses due to each of the individual modes. However, these do not necessarily occur simultaneously. Though it may be tempting to add the maximum responses of each mode directly (this is the sum of absolute values approach), this would lead to unrealistically large estimates of the maximum response. To circumvent this, the maxima from each mode are typically combined using a statistical combination scheme, such as the Square Root of Sum of Squares (SRSS) or Complete Quadratic Combination (CQC). For example, the maximum relative displacement of DOF $j$ in a linear MDOF oscillator with well separated vibration modes can be approximated using an SRSS combination scheme:

$$
\left|u_{j}\right|_{\max }=\sqrt{\sum_{r=1}^{N}\left(\left|u_{j, r}\right|_{\max }\right)^{2}}
$$


in which $u_{j, r}$ is the maximum relative displacement of DOF $j$ due to mode $r$. However, when different vibration modes have closely-spaced frequencies, the CQC approach is more appropriate. Similarly to SDOF systems (equation 2.20), the maximum base shear due to each mode can be obtained from the pseudo-acceleration response spectrum, and the contributions of each mode can be combined to estimate the maximum base shear. As for the mode superposition method, the maximum response can generally be adequately estimated considering a limited number of vibration modes. In practice, it is thus common to use truncated response spectrum analysis.

\subsection{Overview of seismic design provisions in Canada}

As we have seen, the behaviour of a building during earthquake ground motions is a dynamic phenomenon that depends on the characteristics of the ground shaking and the dynamic properties of the building system. Hence, to calculate the maximum seismic effects that a structure is likely to experience within its useful life requires the selection of representative ground motions, as well as the simulation of structural response to these ground motions. Since its first edition in 1941, the National Building Code of Canada (NBCC) has aimed to provide simple guidelines, based on fundamental structural dynamics concepts, to determine design seismic loads for building structures. Over the years, these guidelines have evolved to reflect the growing understanding of the causes and regional characteristics of earthquakes, as well as their effects on structures. This evolution has closely followed developments in international codes, and has been described in several papers and reports, including Mitchell et al (2010), Gilles (2008), and Heidebrecht (2003). Let us however briefly summarize the seismic design provisions of the 2005 NBCC. 


\subsubsection{Seismic hazard and the uniform hazard spectrum}

Buildings in different geographic locations are exposed to different levels of seismic activity, or seismic hazard. Further, the characteristics of earthquakes - for instance, the magnitude, frequency content, duration, and propagation of seismic ground motions - also vary depending on the local geological and tectonic setting. For these reasons, the Geological Survey of Canada has developed seismic hazard maps to capture the spatial variation of earthquake ground motions across Canada (Adams and Halchuk, 2003).

In the latest map, which was developed for the 2005 edition of the NBCC, the design ground motion (DGM) is specified as a uniform hazard spectrum (UHS), whose ordinates have a uniform probability of exceedance of two percent in fifty years. These spectral acceleration ordinates represent the maximum acceleration, resulting from earthquake ground motions, that any SDOF oscillator is likely to experience, on average, approximately every 2500 years. The UHS is similar to a pseudoacceleration response spectrum insofar as it corresponds to peak acceleration values for all SDOF oscillators; however, an important distinction must be made between a response spectrum and the UHS. The ordinates in a response spectrum correspond to the maximum response of all SDOF systems if a specific event were to occur. On the other hand, the probability of exceeding a specific UHS spectral ordinate (two percent in fifty years) is calculated considering events of different magnitude and epicentral distance, along with their probability of occurrence. Each spectral ordinate of a UHS therefore involves hazard contributions from all the different scenarios of earthquake magnitude and epicentral distance that could possibly affect the location under consideration. 
Note that the UHS were developed for five percent damped oscillators and for structures located on very dense soil or soft rock (site class C). Therefore, the UHS must be adjusted for different soil conditions. It can also can be adjusted for different structural damping levels (Atkinson and Pierre, 2004), but this is rarely done in practice. Figure 2.1 shows the UHS for Montréal, adjusted for different soil conditions. Of course, the curves vary for different localities, but the general trend of decreasing spectral acceleration values with increasing period is similar. More details on the development of these maps and the values for different Canadian localities can be found in Adams and Halchuk (2003), Adams and Atkinson (2003), and in Appendix C of Division B of the NBCC 2005 (Climatic and Seismic Information for Building Design in Canada).

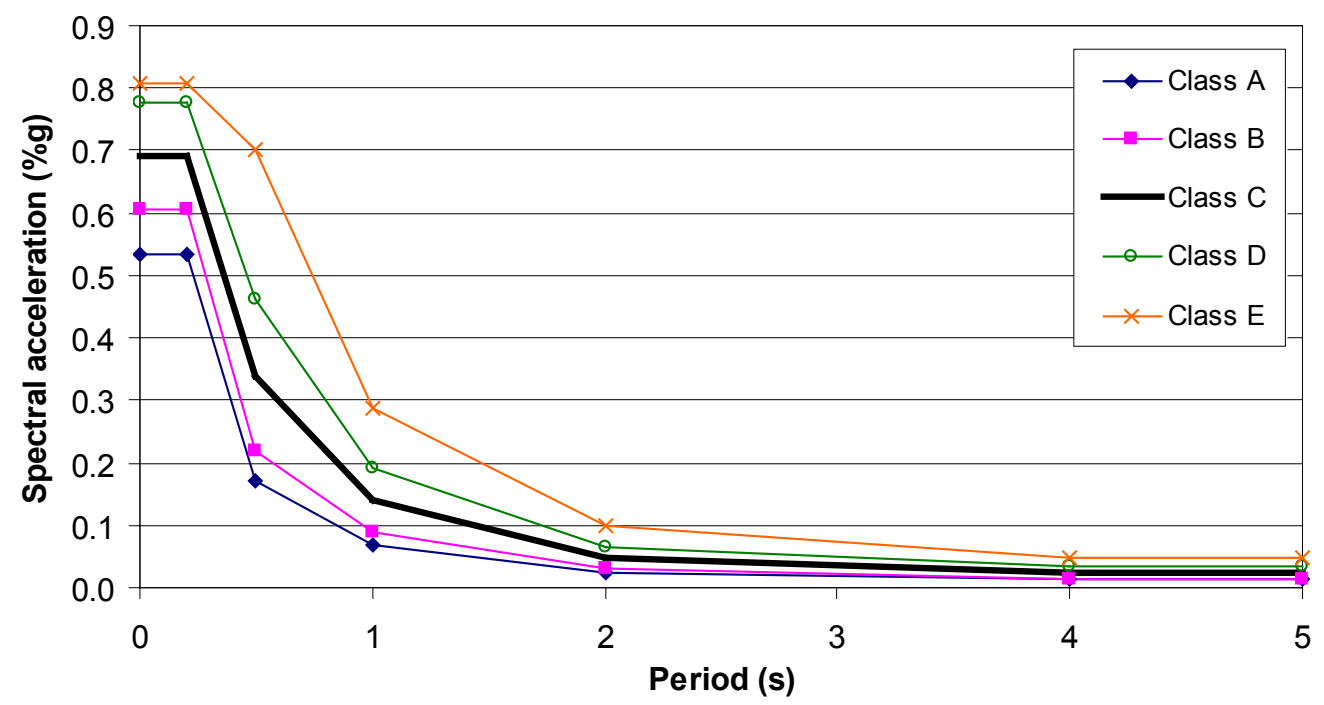

Figure 2.1: Uniform hazard spectrum for Montréal, adjusted for different soil conditions

\subsubsection{Analysis methods}

To estimate the response of a building to the design ground motion, the National Building Code of Canada specifies that dynamic analysis should be used. However, an equivalent static force procedure (ESFP) can be 
used for buildings in low seismicity areas, or buildings that are relatively short and stiff. In such cases, the ESFP adequately captures the maximum response of the structure, or the approximations involved in the ESFP are not likely to have serious consequences (NRC/IRC, 2005b).

\subsubsection{Equivalent static force procedure}

In the ESFP, the design base shear is calculated as

$$
V=\frac{S\left(T_{a}\right) M_{v} I_{E} W}{R_{d} R_{o}}
$$

in which $T_{a}$ is the fundamental period assumed in design, $S\left(T_{a}\right)$ is the design spectral acceleration obtained from the UHS at that period, $M_{v}$ is the higher mode factor, $I_{E}$ is the earthquake importance factor for the structure, $W$ is the total building weight, and $R_{d}$ and $R_{o}$ are the ductility and overstrength force modification factors.

Notice that the form of this equation is very similar to that of equation 2.20 for the base shear of a linear SDOF system. It follows that the ESFP is based primarily on the fundamental mode response of a linear SDOF system. However, a few additional terms have been added to capture the deviation of the behaviour of real buildings from this idealized model. The importance factor simply adjusts the level of safety for a particular structure, depending on its intended use and occupancy and whether it needs to remain in use after an earthquake. The higher mode factor accounts for the fact that, in tall buildings, higher modes typically contribute significantly to the overall response, leading to higher shears at the base of these buildings (Humar and Mahgoub, 2003). The ductilityrelated force modification factor accounts for the fact that most structural 
members have at least some capacity to undergo inelastic deformations, and that seismic forces are reduced when the response is inelastic. Finally, the overstrength-related force modification factor accounts for the dependable reserve strength possessed by structures designed according to the NBCC, by virtue of the rounding of member sizes and dimensions and the differences between nominal and factored resistances, between nominal and design yield strengths, and between the loads required to cause failure of one structural element and those required to reach the collapse capacity of the structure (Mitchell et al., 2003).

The lateral force $V$ is then distributed along the height of the building in an approximately linear fashion, assuming that the masses at each floor level are equal, and that the floor accelerations increase linearly from the ground to the roof level. Torsional effects are also accounted for by applying the forces at each floor level with a specified accidental eccentricity relative to the center of rigidity of that floor, in addition to the torsional moments created by the actual eccentricity between the centre of mass and the centre of rigidity of each floor. This equivalent seismic load distribution is then used to calculate internal forces in structural elements and other response parameters. Note however that displacements calculated from linear elastic analysis with this seismic load distribution must be multiplied by $R_{d} R_{o}$, and divided by $I_{E}$ to provide realistic estimates of the anticipated displacements.

\subsubsection{Dynamic analysis methods}

The complex distribution of forces resulting from earthquake ground motions is better captured by dynamic analysis than by equivalent static procedures. Since dynamic analysis of buildings can now be performed relatively easily in most commercial structural analysis software, it has become the default analysis method in the NBCC, with the use of the 
ESFP being restricted to certain buildings, as described above. Dynamic analysis can be performed using either linear response spectrum analysis, linear time history analysis, or nonlinear dynamic analysis. All three of these methods require structural designers to create a mathematical model of the building, which is generally done using structural analysis software.

In linear response spectrum analysis, the UHS is used as a pseudoacceleration spectrum. The normal vibration modes of the structure are estimated from an eigenvalue analysis, and the corresponding natural periods (or frequencies) are used to read values off the UHS, corresponding to the maximum acceleration for each vibration mode. As explained in section 2.1.9, these values are then combined to estimate the maximum elastic base shear and other response quantities. Note that, as explained in section 2.2.1, the UHS does not truly represent an acceleration response spectrum since it involves hazard contributions from various earthquake scenarios. However, Humar and Mahgoub (2003) showed that using the UHS in response spectrum analysis leads to estimates of design base shear that are not overly conservative.

In linear time history analysis, earthquake acceleration records are input directly at the base of the structure, and the various response quantities (displacements, accelerations, element stresses, etc.) are output at discrete time steps. The input acceleration records must be compatible with the UHS in the range of periods of the modes that contribute significantly to the building's response, and procedures have been developed to obtain such acceleration records (Atkinson, 2009). The output time histories can be studied to extract the maximum values of various responses, such as base shear, overturning moments, floor displacements and accelerations. 
Note that the base shear resulting from either of these linear elastic analysis methods represents the elastic base shear, $V_{e}$. In the same way as the ESFP, this elastic base shear is multiplied by the importance factor, $I_{E}$, and divided by the force modification factors, $R_{d}$ and $R_{o}$ to obtain the dynamic base shear. In this way, the main difference between the dynamic and static analysis methods is reduced to the way in which the effects of higher modes are included in the analysis. However, there is a concern that numerical structural models often underestimate stiffness - for example, by neglecting the stiffening effects of architectural components thus leading to underestimation of base shear in dynamic analysis. To limit the possibility of unrealistically low values being used in design, the design base shear, $V_{d}$, is not permitted to be taken less than 80 percent of the value determined from the equivalent static force procedure (equation 2.35). Further, the elastic storey shears, member forces, and deflections obtained from either linear dynamic analysis method must be multiplied by the ratio $V_{d} / V_{e}$ to obtain their design values. Finally, the design displacements, which now correspond to displacements that were obtained from a linear elastic analysis using reduced forces - which were obtained by dividing the elastic base shear by a factor $R_{d} R_{d} / I_{E}$ - must be re-multiplied by the same factor to provide realistic estimates of the anticipated displacements.

A nonlinear dynamic analysis can also be used to simulate the response of a structure to the design ground motion. In such an analysis, the inelastic behaviour of the various structural elements is modeled directly, based on experimental results from cyclic loading tests of similar elements. Time history analysis is then performed, using ground motion time histories as input, and the time histories of various responses can be extracted. Such analyses generate a considerable amount of data, which need to be scrutinized carefully to ensure that the selected ground motions were able to push the structure into the inelastic range. For this reason, 
such analyses are used more commonly in research than in practice. More details on dynamic analysis can be found in Saatcioglu and Humar (2003).

\subsubsection{Fundamental period determination}

As discussed in the previous section, the fundamental period determines the value of the spectral acceleration used to calculate design base shear using the ESFP. In dynamic analysis, it will determine the contribution of the fundamental translation mode to the overall response. As we have already noted, even when a dynamic analysis is used, the base shear used in design is not permitted to be taken less than 80 percent of the equivalent static base shear. Therefore, the prediction of the fundamental period of a building is an important step to calculate design seismic loads according to the provisions of the NBCC. To this end, the NBCC provides the following empirical equations to determine the fundamental period for different types of structural systems:

$$
\begin{array}{ll}
T_{1}=0.085 h^{3 / 4} & \text { for steel moment-resisting frames, } \\
T_{1}=0.075 h^{3 / 4} & \text { for reinforced concrete moment-resisting frames, (2.37) } \\
T_{1}=0.1 \mathrm{~N} & \text { for other moment-resisting frames, } \\
T_{1}=0.025 \mathrm{~h} & \text { for steel braced frames, and } \\
T_{1}=0.05 \mathrm{~h}^{3 / 4} & \text { for shear wall and other structures }
\end{array}
$$

in which $h$ is the building height above ground in $\mathrm{m}, N$ is the number of storeys, and $T_{1}$ is the fundamental translation period in $\mathrm{s}$.

In the ESFP, the code allows structural designers to use other methods for instance, eigenvalue analysis - to determine the fundamental period, but it limits the deviation from the value calculated from the appropriate 
equation shown above. For RCSW buildings, the maximum period that can be used is twice that calculated from equation 2.40. In dynamic analysis, no restrictions are imposed on the fundamental period per se; as mentioned above, these restrictions are instead applied to the design base shear.

It is important to note that the NBCC does not provide any guidance with regards to the periods of torsion or higher vibration modes that should be used in dynamic analysis.

Let us now focus specifically on the design of reinforced concrete shear wall buildings. The next section will discuss in more detail how equation 2.40 was derived, and different models to estimate the periods of torsion and higher translation modes and modal damping will also be discussed.

\subsection{Dynamic properties of RCSW buildings}

As we have seen, the dynamic properties of a building - namely, its natural frequencies, mode shapes, and damping - play a prominent role in determining its response to earthquake ground motions. In response spectrum analysis, the natural frequencies of several vibration modes are required to read off spectral acceleration values from response spectra (or uniform hazard design spectra). Similarly, in time history analysis, the response is often obtained by superimposing the responses in several vibration modes. In the equivalent static force procedure, the fundamental period determines the value of the spectral acceleration used to calculate design base shear. Therefore, predicting these dynamic properties is an important step in determining design seismic forces. Similarly, if dynamic analysis is used to predict the response of a building to wind loads - for instance, in tall buildings - then the estimation of its dynamic properties is equally important. 
Let us therefore review different models that have been suggested over the years to predict the dynamic properties of RCSW buildings. These models are evaluated in section 5 using the dynamic properties of 27 RCSW buildings in Montréal identified from ambient vibration records.

\subsubsection{Fundamental period}

The fundamental translation mode (in each direction) generally contributes the most to seismic building response, particularly in short buildings. For this reason, experimental research and seismic recommendations in building codes have focused mainly on the estimation of the period of the fundamental translation mode.

The fundamental period of a building is a function of its lateral stiffness and mass, as well as their spatial distribution. Many parameters contribute to lateral stiffness and mass, most notably the type of structural system (particularly, the types of structural elements that are designed to resist lateral loads), the dimensions and number of these structural elements, as well as the building height. For this reason, different empirical relationships have been developed to predict the fundamental period for buildings having different lateral load-resisting systems. These range in complexity from simple equations based solely on building height (ASCE, 2005; Farsi and Bard, 2004; Fritz et al., 2009; Lagomarsino, 1993; NRC/IRC, 2005a; Saatcioglu and Humar, 2003) to more complex equations which incorporate the dimensions of individual structural elements (Goel and Chopra, 1998; Lee et al., 2000; Morales, 2000; Wallace and Moehle, 1992). Simple equations based solely on building height cannot be expected to be very accurate since they neglect many parameters that contribute to a building's lateral stiffness; however, they are easy to apply in general. In contrast, more complex equations that incorporate the 
dimensions of lateral load-resisting elements, may in certain cases be more accurate, but they may only apply to very simple buildings because of the assumptions required to develop them.

Several additional considerations, which are typically ignored in empirical relationships, likely affect the fundamental period of building systems. First, both simple and more complex equations generally ignore the stiffening effects of architectural components (Ventura and Schuster, 1996), as well as the effects of soil conditions at the site and foundation details (Ghrib and Mamedov, 2004). Further, there is a concern that local design and construction practices, as well as seismic hazard, play a significant role in the lateral stiffness of a building, which may lead to regional variations in the fundamental periods of similar buildings (Hong and Hwang, 2000). Further compounding the difficulties in estimating the fundamental period of a building is the fact that the period is not a fixed parameter. That is, the fundamental period of a single structure has been shown to elongate with the level of excitation (McVerry, 1979; Trifunac et al., 2001b). Thus, accurately simulating the response of a particular building to both design-level winds, and design-level earthquake ground motions, could require the use of different fundamental period estimates, selected based on the expected magnitude of each event. However, no widely accepted methods are available to quantify the relationship between excitation magnitude and period elongation.

Bearing in mind these challenges, let us concentrate on the more common models that have been developed over the years to estimate the fundamental period of RCSW buildings. In section 5.1, these are evaluated using the measured fundamental periods of RCSW buildings in Montréal. 


\subsubsection{Code formula}

To estimate the fundamental period of RCSW buildings, equation 2.40 was adopted in the 2005 edition of the NBCC based on the recommendations of Saatcioglu and Humar (2003). This equation has figured in American codes since the late 1980s and remains in use in several of them (ASCE, 2005; BSSC, 2003; SEAOC, 1999). The form of this equation, with the exponent of $3 / 4$ applied to the building height, was derived during the development of ATC 3-06 Tentative provisions for the development of seismic regulations for buildings (ATC, 1978), using Rayleigh's method. This method is commonly used to estimate the fundamental period of a building by making an assumption on the shape of the corresponding vibration mode that satisfies the displacement boundary conditions at the supports. This can be done by using the deformed shape resulting from an assumed static lateral load distribution, as this will automatically satisfy these boundary conditions (Chopra, 2001). In this case, the following assumptions were made:

- The base shear (or total horizontal force) was assumed to vary as $1 / T^{2 / 3}$ for convenience since seismic design spectra varied in this manner in ATC 3-06,

- The distribution of lateral forces was assumed to vary linearly from zero at the base to a maximum at the roof level,

- The resulting deformed shape was also assumed to vary linearly from zero at the base to a maximum at the roof level, essentially assuming the fundamental mode shape to be linear, and

- Masses at each storey level were assumed to be equal.

Note however that that the form of this equation, particularly the value of the exponent, would be different if different, but equally plausible, assumptions were made in the analysis. The interested reader may refer 
to Goel and Chopra (1997, Appendix D) for additional details on the derivation of this equation. Finally, it appears that the coefficient of 0.05 was obtained by regression analysis, using the fundamental periods of nine RCSW buildings in California measured during the 1971 San Fernando earthquake (ATC, 1978; SEAOC, 1999).

As evident in Figure 2.2 below, which shows the periods of 16 RCSW buildings presented in a later study, as well as the code period equation and the maximum period from a rational analysis that is allowed by the NBCC (see section 2.2.3), the measured data are quite scattered and the code equation does not fit the data particularly well. Further, since the coefficient was calibrated using period data from buildings in California, it is unclear whether this equation reflects the fundamental periods of RCSW buildings in Eastern Canada. For these reasons, it is important to evaluate this equation using the measured periods of Canadian buildings.

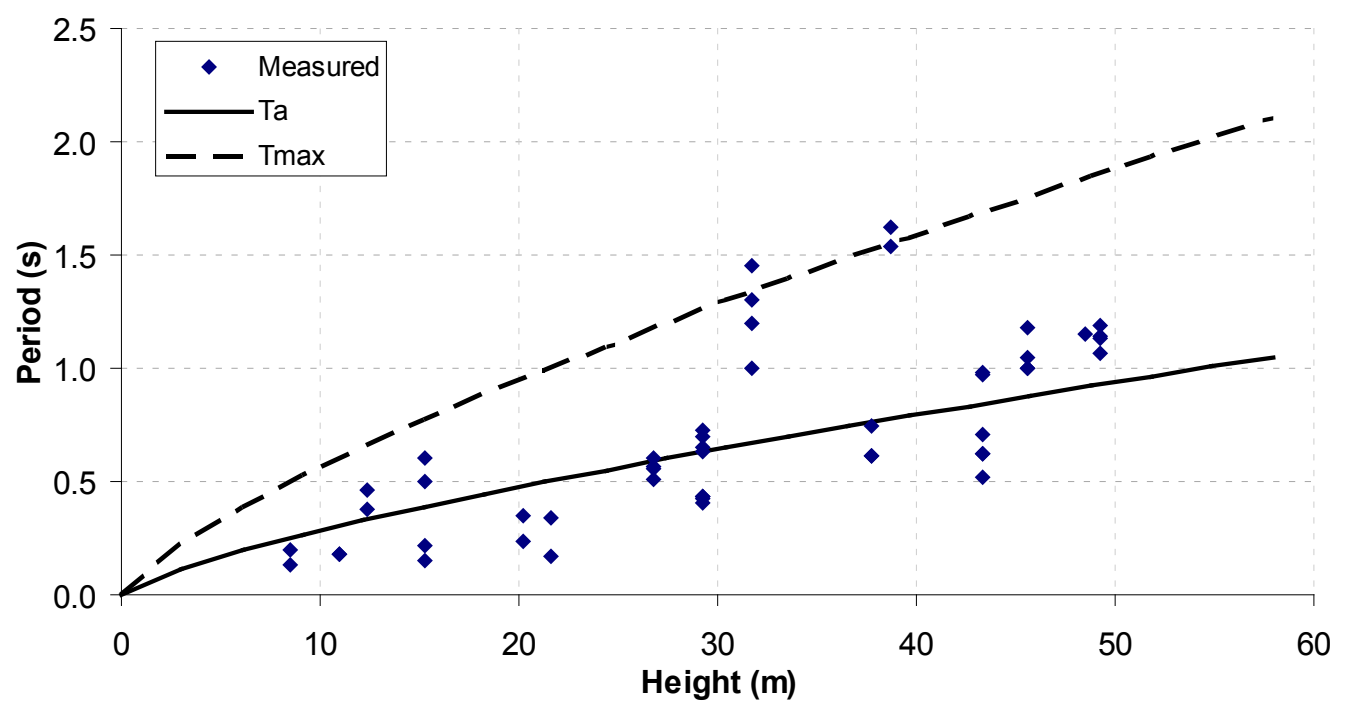

Figure 2.2: Comparison of measured and code fundamental periods for RCSW buildings in California (Source: Goel and Chopra (1998)) 


\subsubsection{Alternative formulae based on building height}

Several other empirical relationships, based solely on building height or the number of storeys, have been suggested by other authors for similar buildings in various parts of the world.

Farsi and Bard (2004) performed ambient vibration tests in 35 RCSW buildings in the Grenoble region, in France. Using the fundamental periods of these buildings identified from the ambient vibration records, they suggested that the fundamental period of RCSW buildings could be estimated reasonably well using

$$
T_{1}=0.01 \mathrm{~h}
$$

They further noted, similarly to Hong and Hwang (2000), that this equation may not apply to RCSW buildings in other regions.

Similarly, using the fundamental periods of $52 \mathrm{RC}$ buildings in Italy identified from ambient vibration records, Lagomarsino (1993) suggested the following equation to estimate the fundamental period of such buildings:

$$
T_{1}=0.018 h
$$

Note however that no distinction was made between RC buildings with and without shear walls in the above equation, which may explain the longer periods predicted, as compared to equation 2.41 . 


\subsubsection{Goel and Chopra formula}

Recognizing that equation 2.40 was based on a limited amount of data, and that recorded motions of buildings during more recent events were available, Goel and Chopra (1998) set out to evaluate this equation using the periods of 16 RCSW buildings identified from their recorded seismic motions. Figure 2.2 shows how the fundamental periods of these 16 buildings compare to the predictions of equation 2.40 .

They found that this equation was inadequate, often leading to overestimates of the fundamental period, which is unconservative for the prediction of seismic base shear. They suggested that the building height alone is not sufficient to accurately predict the fundamental period since it does not capture the increased stiffness contributed by additional walls or walls of larger dimensions. To address this, they proposed a more complex equation, making use of the dimensions of shear walls, which was derived considering flexural and shear deformations of a uniform cantilever, and calibrated to the measured periods of a subset of nine buildings, for which shear wall dimensions were available. This equation was incorporated as an alternative equation for RCSW buildings in several international codes (ASCE, 2005; BSSC, 2003) and is given by

$$
T_{1}=\frac{0.0062 h}{\sqrt{A_{e}}}
$$

where

$$
A_{e}=\frac{100}{A_{B}} \sum_{i=1}^{N W}\left(\frac{h}{h_{i}}\right)^{2} \frac{A_{i}}{\left[1+0.83\left(\frac{h_{i}}{D_{i}}\right)^{2}\right]}
$$


In the above equations, $A_{i}, h_{i}$, and $D_{i}$ respectively represent the plan area, height, and width of each shear wall aligned with the direction under consideration, $N W$ represents the total number of shear walls in the same direction, $A_{B}$ represents the building plan area, $A_{e}$ represents the equivalent shear area, $h$ represents the total building height in metres, and $T_{1}$ represents the fundamental period in seconds.

It should be noted that in deriving the above equations, the following simplifying assumptions were made:

- All shear walls are uncoupled,

- The building plan is symmetric in the lateral direction under consideration and does not change along the building height, and

- The shear walls have the same dimensions (width and thickness) along the building height.

Figure 2.3 shows how the periods of the subset of nine buildings compare to those predicted using equation 2.43. Three curves are shown, corresponding to the best-fit from regression analysis ( $T_{R}-$ thin, middle brown line), best-fit minus one standard deviation ( $T_{L}-$ thick, lower black line), and best-fit plus one standard deviation ( $T_{U}-$ dashed, upper blue line). 


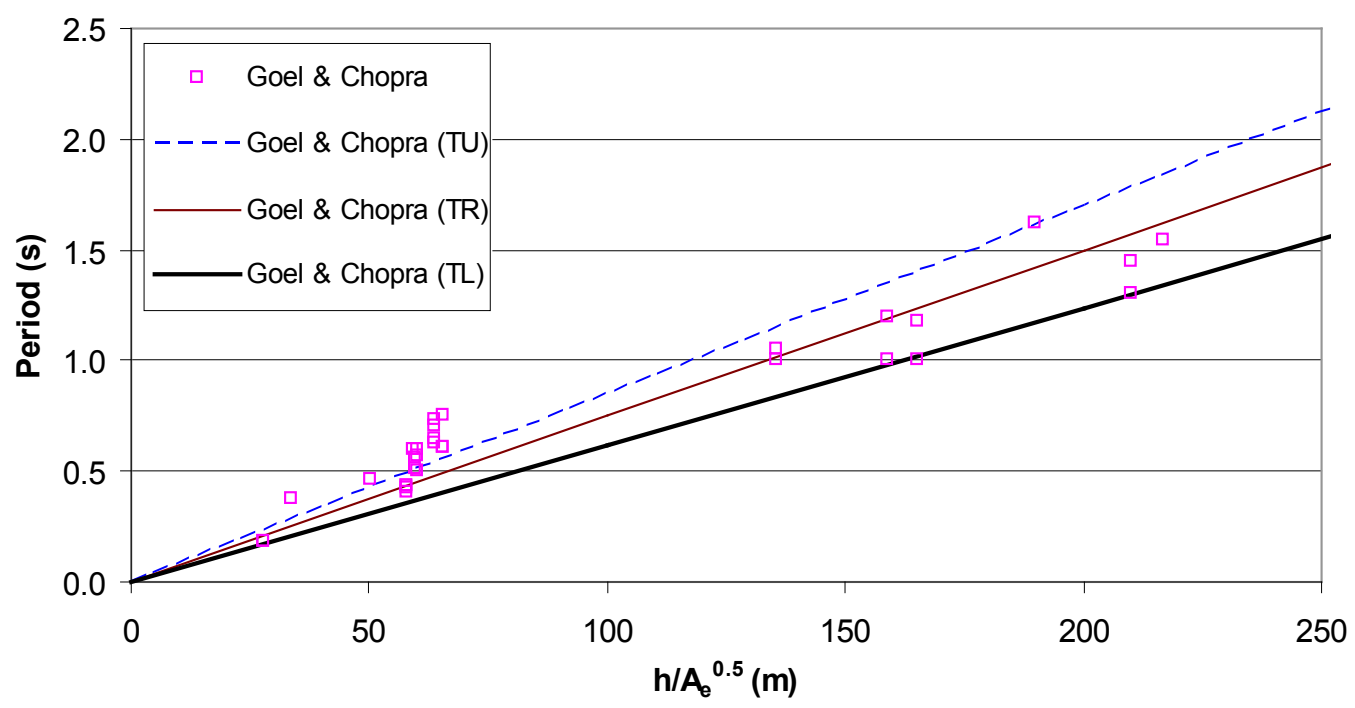

Figure 2.3: Goel and Chopra fundamental period formula

Note that equation 2.43 corresponds to the best-fit minus one standard deviation $\left(T_{L}\right)$, and was calibrated to generally underestimate the fundamental period, thus leading to conservative estimates of design base shear in a majority of cases. Conversely, in a later paper, Chopra and Goel (2000) suggested that the best-fit plus one standard deviation curve $\left(T_{U}\right)$ should be used to calculate seismic displacements, since these generally increase with increasing period.

Though this formulation appears to reduce the variability in period data, it should be noted that it requires a priori knowledge of shear wall dimensions. Further, as we will see in section 5.1.6.1, a significant inconsistency is observed in equation 2.44 when some shear walls do not extend all the way to the top of a building.

\subsubsection{Wallace and Moehle formula}

Wallace and Moehle (1992) studied the fundamental period of a uniform concrete cantilever of rectangular cross section, supporting regularly 
distributed floor loads, considering only flexural deformations. Based on a theoretical analysis with assumed values of floor weight, concrete modulus of elasticity, and storey height, they suggested computing the fundamental period of uncracked reinforced concrete shear wall buildings using

$$
T_{1}=0.0019 \frac{h_{w}}{D} \frac{n}{\sqrt{p}}
$$

where $h_{w}$ represents the wall height, $D$ represents the wall width, $n$ represents the number of storeys, and $p$ represents the ratio of total wall area to floor plan area for the walls aligned with the lateral direction under consideration. In contrast, considering cracked-section stiffness to be equal to half the gross-section stiffness, they suggested

$$
T_{1}=0.0027 \frac{h_{w}}{D} \frac{n}{\sqrt{p}}
$$

to estimate the fundamental period of cracked reinforced concrete shear wall buildings.

According to the authors, periods measured from small amplitude ambient motions should compare well with equation 2.45, while periods from moderate seismic motions should compare well with equation 2.46 .

\subsubsection{Lee et al formula}

Similarly to Goel and Chopra (1998), Lee et al (2000) derived a theoretical model to predict the fundamental period of a uniform cantilever, considering both flexural and shear deformations; however, they assumed the thickness of all walls to be identical. The model parameters were 
obtained from regression analysis, using the fundamental periods of 50 reinforced concrete shear wall buildings in Korea, identified from ambient vibration records. This led to the following equation:

$$
T_{1}=0.4\left(\frac{h^{0.2}}{\sqrt{L_{w}}}\right)-0.5
$$

where $L_{w}$ represents the total wall width per unit plan area (in $\mathrm{m}^{-1}$ ), and is calculated as follows:

$$
L_{w}=\frac{\sum_{i=1}^{N W} D_{i}}{A_{B}}
$$

\subsubsection{Morales formula}

Finally, Morales (2000) recognized that the fundamental period of a uniform flexural cantilever (neglecting shear deformations) is dependent on the ratio of the height to the second moment of area. She suggested that the second moment of area of a reinforced concrete building with shear walls, in either principal direction, could be approximated as the summation of the second moments of area of individual walls, in the same direction. She then examined different expressions, involving both the height and second moment of area, to determine whether these could improve the prediction of the fundamental period of reinforced concrete shear wall buildings. The equations considered were of the form

$$
T_{1}=a \frac{h^{\alpha}}{I^{\beta}}+b
$$


where $h$ represents the building height, $I$ represents the second moment of area of the building in the direction under consideration (calculated as the sum of the second moments of individual walls in the same direction), and the remaining coefficients are constants. Different values of $\alpha$ and $\beta$ were considered, and the coefficients $a$ and $b$ were obtained by regression analysis using the measured periods of 18 reinforced concrete shear wall buildings in California. Note that these data are for the most part identical to the data used by Goel and Chopra in developing equation 2.43, and were obtained during moderate earthquake ground motions.

The equation that produced the best fit to the measured data was found to be

$$
T_{1}=0.13 \frac{h}{I^{0.25}}-0.4
$$

\subsubsection{Other factors affecting the fundamental period}

As mentioned previously, fundamental building periods are likely affected by a number of other factors, which may explain the variability observed in the fundamental period data presented above. First, non-structural (architectural) components may add to the lateral stiffness of a building, leading to slightly shorter periods. Ventura and Schuster (1996) studied the changes in the dynamic properties during the construction of a reinforced concrete high-rise building in Vancouver, and found that its fundamental periods decreased by a maximum of only three percent (relative to the bare frame) after all non-structural elements were added. Similarly, based on ambient tests performed before and after the removal of non-structural components in five RCSW buildings in France under demolition, Hans et al (2005) reported an increase in natural periods of two to four percent for the bare structure. Hence, non-structural 
components do not appear to significantly affect the lateral stiffness of reinforced concrete buildings, but more evidence is needed to confirm whether this can be generally accepted.

Next, there is a concern that differences in design and construction practices, as well as differences in seismicity, may cause regional variations in the fundamental periods of similar buildings. The rationale is that these factors may affect the overall lateral stiffness through the use of different building materials and construction techniques, or through the use of smaller lateral load-resisting elements in areas of lower seismicity. This however is very difficult to assess in practice without very large data sets of similar buildings in different areas. For this reason, researchers have typically been content to mention that fundamental period relations developed based on data from buildings in a given region should be used with care in other locations (Farsi and Bard, 2004; Goel and Chopra, 1998; Hong and Hwang, 2000).

Moreover, fundamental periods identified from tests in real buildings generally reflect the overall lateral stiffness of the building system, including the building itself (including non-structural components and contents), its foundation, and the underlying soil. Ghrib and Mamedov (2004) suggested that differences in foundation flexibility could explain the variability observed in period data from real buildings. They used the measured ambient responses of 20 buildings in Baku, Azerbaidjan, on different soils, to calibrate an analytical fundamental period model that includes the properties of both the structure and the underlying soil. However, this model is rather complex and, for this reason, has not yet been widely adopted.

Finally, several studies have shown that the fundamental periods of a building tend to increase with the level of excitation, from ambient to 
strong motions (Çelebi, 1996; Li and Mau, 1997; McVerry, 1979; Trifunac et al., 2001a; Trifunac et al., 2001b). This has generally been attributed to changes in effective building stiffness (due to cracking of RC members or participation of non-structural elements), foundation stiffness (due to softening of soils at large strains), or structural damage. However, the changes in fundamental periods, from ambient to strong motions, are not consistent for all buildings. For this reason, no widely accepted method exists to quantify the period elongation that can be expected from ambient to strong motions.

In summary, the equation currently adopted in the National Building Code of Canada to estimate the fundamental period of RCSW buildings is based on a relatively poor fit to the measured fundamental periods of buildings in California. Therefore, it is of paramount importance to evaluate the accuracy of the code equation using the measured periods of real buildings in Canada. Similarly, alternative equations must be considered to see whether these can provide more accurate estimates of the fundamental period. In section 5.1, the equations presented above are evaluated using the measured fundamental period of RCSW buildings in Montréal.

\subsubsection{Damping}

As we have seen, the capacity of a building to dissipate energy during vibration is vital to reducing the vibration amplitudes during dynamic loading events, such as strong winds or earthquake ground motions. Fortunately, all structures possess inherent damping through a variety of mechanisms, such as material damping, friction in joints and between structural and non-structural components, and radiation of energy into the underlying soil. However, due to the varied nature of these mechanisms, damping is not rigorously modeled in structural analysis of buildings. 
Instead, simple damping models that are selected based on mathematical convenience rather than accuracy, such as Rayleigh damping, in which the damping matrix is proportional to the stiffness and mass matrices, or constant modal viscous damping, are typically used.

In seismic analysis and design, it is most common to assume five percent viscous damping in all significant low frequency vibration modes. In fact, seismic design spectra in most modern building codes are specified for five percent viscously damped oscillators. In contrast, for wind applications, the use of lower damping values is generally recommended to assess serviceability performance (NRC/IRC, 2005b). As we will see, one of the reasons for this discrepancy is that measured damping in real buildings has been shown to appreciably increase with the amplitude of building motion. Therefore, the amount of damping that can be expected during design-level earthquake shaking is significantly larger than during strong winds.

These recommendations are based on damping values that have been measured in different types of structures. Indeed, over the years, several efforts have been undertaken to measure damping in real buildings and use these data to develop models to refine the estimation of damping at the design stage. In most of these models, damping varies with natural frequency (or height) and, in certain cases, vibration amplitude. However, measured damping values vary widely - even for seemingly similar buildings - and none of these models significantly reduces this variability. Therefore, none of these models has achieved general consensus and engineers continue to use simplified methods to account for damping in structural analysis. Nevertheless, the most common damping models are presented here. In section 5.2, their accuracy will be evaluated using the measured damping values identified from ambient tests in RCSW buildings in Montréal. 
Jeary (1986) suggested that the main mechanism of energy dissipation in large structures is friction between elements and internally in the materials. Further, he noted that measured damping values were dependent on the amplitude of vibration, and that this likely resulted from an increase in radiation damping in the foundation. He proposed that the prediction of damping should be characterized by two terms: a lowamplitude damping value, and an additional term accounting for the increase of damping with vibration amplitude, as follows:

$$
\xi_{j}=\xi_{o j}+\xi_{1 j} \frac{x_{h}}{h}
$$

where $\xi_{j}, \xi_{0 j}$, and $\xi_{l j}$ are the total damping ratio (as percentage of critical viscous damping), the low-amplitude damping ratio, and the rate of change of damping with vibration amplitude, corresponding to mode $j, x_{h}$ is the maximum displacement at the top of the building, and $h$ is the building height.

Using measured damping values for the fundamental translation modes of nine reinforced concrete buildings, he suggested that the low-amplitude damping was correlated to the natural frequency of the corresponding mode, and that the rate of change of damping with vibration amplitude depended on the width of the building in the direction under consideration, presumably due to an increase in radiation damping for wider buildings. He thus proposed the following model to estimate damping:

$$
\xi_{j}=f_{o j}+10^{\sqrt{D_{B}} / 2} \frac{x_{h}}{h}
$$


where $f_{0 j}$ is the low-amplitude natural frequency (in $\mathrm{Hz}$ ) corresponding to mode $j$, and $D_{B}$ is the dimension of the building at the base (in $\mathrm{m}$ ) in the direction under consideration.

In the same vein, Lagomarsino (1993) suggested a more detailed model to predict damping in reinforced concrete buildings at low vibration amplitudes, which was developed based on regression analysis considering the measured damping values of 52 reinforced concrete buildings in their six lowest frequency modes - two translation modes in each principal direction and two torsion modes.

$$
\xi_{0 j}=\frac{0.7238}{f_{0 j}}+0.7026 f_{0 j}
$$

Later, Satake et al (2003) used damping data from 25 reinforced concrete buildings in Japan, obtained for the most part from ambient vibration tests, to develop improved empirical models to predict damping. Based on regression analyses, they proposed that the damping ratio of the fundamental translational mode corresponding to low amplitudes of vibration could be estimated using either the building height or the fundamental frequency:

$$
\begin{aligned}
& \xi_{1}=\frac{98.9}{h}, \text { or } \\
& \xi_{1}=1.38 f_{1}
\end{aligned}
$$

Using the data for which vibration amplitudes were known, they suggested a simpler equation to that proposed by Jeary to take into account the effect of vibration amplitude on fundamental translational mode damping, which was 


$$
\xi_{1}=100\left(0.014 f_{1}+470 \frac{x_{h}}{h}-0.0018\right)
$$

To estimate damping in the fundamental torsion mode, they suggested a slightly different equation than for the fundamental translation mode:

$$
\xi_{1 t}=0.95 f_{1 t}
$$

where $\xi_{l t}$ and $f_{l t}$ represent the damping ratio and natural frequency of the first torsion mode.

Finally, they found that damping in higher modes tended to increase with the mode number. Considering the experimental damping ratios for the higher vibration modes of the 25 buildings studied, they concluded that these could be reasonably predicted from

$$
\xi_{j}=1.4 \xi_{j-1}
$$

where $\xi_{j}$ and $\xi_{j-1}$ represent the damping ratios in two successive vibration modes in the same direction.

Though this was not included in their models, the authors also found that building use seemed to affect damping, with slightly larger values for hotels and apartment buildings. To explain this, they suggested that an increased number of partition walls in these types of buildings, compared to office buildings, increases damping, presumably by increasing the amount of interfacial friction losses. Conversely, foundation type, building length and width, and floor area, were not found to be correlated to damping at low vibration amplitudes. 
Based on the data used to develop them, all the models described above were characterized by significant dispersion of the data and very weak correlations. Further, though the amplitude-dependent nature of damping was stressed in all the above studies, the data used to calibrate the models were mostly obtained from ambient tests having relatively low vibration amplitudes.

To determine the amount of damping that can be expected during earthquake ground motions, Çelebi (1996) compared damping values obtained from strong motion records with those from ambient tests for five buildings in the San Francisco area, three of which were reinforced concrete buildings. The strong motion data were obtained during the Loma Prieta earthquake, while the ambient data were obtained from tests performed after the earthquake. As expected, damping was significantly smaller during ambient motions than during earthquake ground motions for all five buildings. However, the difference between damping values during ambient and strong motions was not consistent for all buildings, with damping in certain modes increasing approximately five-fold from ambient to strong motions, while they only increased by approximately fifty percent in others.

Building on these findings, $\mathrm{Li}$ and $\mathrm{Mau}$ (1997) attempted to correlate damping to ground excitation, response acceleration, and storey drift, using the measured responses of seven reinforced concrete shear wall, nine reinforced concrete moment-resisting frame, and five steel momentresisting frame buildings during the Loma Prieta and Whittier earthquakes; but no convincing correlation could be found.

Finally, Fritz et al (2009) compiled a comprehensive database of damping measurements from vibration tests performed around the world. Using statistical techniques, they found that the most relevant factors affecting 
damping were building height, material, and level of excitation. Comparing the data from earthquake tests with those from ambient tests, they found that, on average, damping during earthquake ground motions could be expected to be twice that during ambient motions. Moreover, they developed the following model to predict damping in the fundamental mode for reinforced concrete buildings:

$$
\xi_{1}=\frac{14}{\sqrt[4]{n}}
$$

where $n$ represents the number of storeys. However, it is not clear whether this model was meant to represent low-amplitude damping or damping during moderate to strong earthquake ground motions.

It is important to note that energy dissipation due to yielding of structural members during strong motions is already accounted for in seismic analysis and design: in nonlinear time history analyses, hysteretic material models are typically specified for the various elements of the structural framework, while in static methods, an R-factor accounting for the ductility of the lateral load-resisting system is used to reduce the lateral forces on the structure. Clearly then, energy dissipation associated with this mechanism should not be used a second time to increase the damping ratios used in structural analysis (PEER/ATC, 2010). Further, it is not clear whether the damping values measured during past seismic events were obtained from structures that underwent significant yielding. In part due to this, the Council on Tall Buildings and Urban Habitat (CTBUH) recommends the use of damping values between one and two percent for the seismic design of buildings more than $50 \mathrm{~m}$ and less than $250 \mathrm{~m}$ high (CTBUH, 2008). 
Bearing in mind the uncertainty associated with modeling damping, it is important to evaluate whether common assumptions regarding this parameter (i.e. five percent viscous damping in all vibration modes) truly reflect the level of energy dissipation that can be expected once a building is constructed. In section 5.2, the measured damping values in RCSW buildings in Montréal are used to evaluate the various damping models.

\subsubsection{Natural periods of torsion and second translation modes}

As we have seen, the fundamental translation mode (in each direction) generally contributes the most to seismic response, and experimental research and recommendations in building codes have focused mainly on the estimation of the period and damping of the fundamental translation mode. However, it has long been recognized that higher modes may play a significant role in dynamic response to strong winds and earthquakes, particularly for tall structures having long fundamental periods (Humar and Mahgoub, 2003). Furthermore, when torsion modes are excited, the torsional response of a structure can lead to increased stresses and strains in certain structural elements.

As computer-aided design tools are now widely used by structural engineers, dynamic analyses can be relatively easily performed. In fact, dynamic analysis is now the preferred method for the seismic design of buildings in Canada, while the use of the equivalent static force procedure is restricted to simple buildings, not exceeding $60 \mathrm{~m}$ in height. When dynamic analyses are used, engineers must estimate the natural periods for several vibration modes, including both translation and torsion modes. This is usually done implicitly by eigenvalue analysis modules within computer programs used for structural analysis. However, the periods thus obtained can vary widely, depending on the analysis assumptions. Unfortunately, little guidance is provided, in building codes or elsewhere, 
to evaluate how the estimated values compare to the measured periods of these vibration modes for similar buildings.

It is therefore important to provide such guidance based on the periods of real buildings. To this end, let us here review two studies that have examined the natural periods of torsion and second translation modes in reinforced concrete buildings. In section 5.3, we build on these studies considering period data from RCSW buildings in Montréal.

Lagomarsino (1993) studied the natural periods of 52 reinforced concrete buildings in their fundamental torsion mode, as well as in their second and third translation modes in each direction. Similarly, Satake et al (2003) studied the natural periods of the same vibration modes for 25 reinforced concrete buildings in Japan. Note that no distinction was made between buildings with and without shear walls in either of these studies.

With regards to the natural period of the fundamental torsion mode of RC buildings, Lagomarsino suggested that it could be calculated based on building height, while Satake et al proposed a model based on the fundamental translation period:

$$
\begin{array}{ll}
\text { Lagomarsino (1993): } & T_{1 t}=0.017 \mathrm{~h} \\
\text { Satake et al (2003): } & T_{1 t}=0.77 T_{1}
\end{array}
$$

where $T_{1 t}$ is the fundamental torsion period in seconds, $T_{1}$ is the fundamental translation period in $\mathrm{s}$, and $h$ is the building height in $\mathrm{m}$.

Theoretically, the ratio of the period of the second translation mode to that of the fundamental mode is 0.333 for a shear cantilever (Newmark and Rosenblueth, 1971) and 0.159 for a flexural cantilever (Chopra, 2001). Hence, the second translation mode period of RC buildings has been 
related to that of the corresponding fundamental translation mode in previous work:

$$
\begin{array}{ll}
\text { Lagomarsino (1993): } & T_{2}=0.27 T_{1} \\
\text { Satake et al (2003): } & T_{2}=0.31 T_{1}
\end{array}
$$

where $T_{2}$ is the natural period of the second translation mode. Since the coefficients in both these equations approach that of a shear cantilever, these expressions suggest that the behaviour of $\mathrm{RC}$ buildings is similar to that of a shear cantilever, at least with respect to the ratio of the first two translation periods.

Finally, to the best of the author's knowledge, little work has been done regarding the natural period of the second torsion mode of buildings. Considering the results of past studies of the other vibration modes, it is reasonable to expect that the second torsion period should be correlated with both the building height and the fundamental torsion period. This, along with the models suggested for the other vibration modes discussed above, is evaluated in section 5.3.

\subsection{Experimental modal analysis and ambient vibration testing}

As mentioned in the introduction, the main objective of this study is to evaluate how common methods used to estimate the natural periods and damping characteristics of buildings compare to the dynamic properties of real buildings. To this end, ambient vibration measurements were performed in 39 buildings, 27 of which were RCSW buildings. Let us now discuss the relevant theoretical and practical aspects involved in the identification of dynamic properties of real structures from vibration 
measurements, a field commonly referred to as experimental modal analysis (EMA). As we will see, ambient vibration tests are an attractive option for large civil engineering structures; therefore, we focus particularly on these types of tests.

\subsubsection{Forced vibration tests}

Traditionally, EMA has been performed in the context of forced vibration tests, the simplest of which involve exciting a structure with a known input at a specific degree of freedom (DOF), $f_{k}(t)$, and measuring a single response component at a particular DOF, $x_{j}(t)$. This is referred to as a single-input-single-output (SISO) modal test. Recall from equation 2.31 that the input and output at any two DOF can be related through a frequency response function (FRF), which depends on the natural frequencies, mode shapes, and damping of the oscillator. Conversely, if the input is known and the output is measured, the FRF relating the input to the output, can be estimated by computing the ratio of the output and input Fourier spectra (Maia et al., 1997):

$$
H_{j k}(\omega)=\frac{X_{j}(\omega)}{F_{k}(\omega)}
$$

However, it is more common to estimate the FRF using spectral densities, rather than Fourier spectra. The estimation of spectral density is discussed in more detail in section 2.5.3. Suffice it to say here that the spectral density between two time signals provides a measure of the energy content per unit frequency in these signals. Two common FRF estimators are denoted $H_{j k, 1}(\omega)$ and $H_{j k, 2}(\omega)$ and given by 


$$
\begin{aligned}
& H_{j k, 1}(\omega)=\frac{G_{F k X j}(\omega)}{G_{F k F k}(\omega)} \\
& H_{j k, 2}(\omega)=\frac{G_{X j X j}(\omega)}{G_{X j F k}(\omega)}
\end{aligned}
$$

where $G_{F k X j}(\omega)$ is the spectral density between the input at DOF $k$ and the output at DOF $j$, and similarly for the other terms.

Depending on the application and the available test equipment, the excitation itself can vary in character, from a harmonic excitation (which excites a particular frequency), to a transient force (for instance from an impact hammer), to white noise (exciting all frequencies approximately equally). For example, a sine sweep SISO test can be used to identify the dynamic parameters of a system by slowly varying the frequency of a harmonic excitation until the response is amplified. Evidently, it is also possible to excite several DOF with different forces, and to measure the responses at different DOF, giving rise to multiple-input-multiple-output (MIMO) tests.

A number of different algorithms have been developed over the years to obtain the dynamic parameters of a structure from forced vibration tests. In general, these are separated into time domain and frequency domain identification methods. A further category, known as subspace methods, relies on a state-state formulation of the equations relating the inputs and the outputs (Maia et al., 1997). Forced vibration testing methods, as well as the different algorithms to extract the dynamic parameters from such tests, are well documented in several books on EMA; two of the more notable being Ewins (2000) and Maia et al (1997). 
The main advantage of forced vibration tests is that both the system response and the input forces are known. It is thus possible to obtain reliable estimates of the modal parameters using the FRF estimators. However, artificially exciting large civil engineering structures requires large shakers, which can make such tests time-consuming and labourintensive. Furthermore, in such structures, ambient excitation sources for instance from wind - are always present in addition to the controlled input forces, thereby compromising the input-output relationship (Parloo et al., 2003).

\subsubsection{Free response tests}

Free response tests involve subjecting a structure to a set of initial conditions and measuring its response over time. For instance, this can be done by displacing the structure according to a particular deformation pattern and releasing it. Alternatively, the structure can be impacted to produce initial conditions on velocity, rather than displacement. Or, the structure can be excited at a particular frequency until the response amplitudes are large enough, and then the excitation removed and the free vibration response measured (Schiff, 1972). If the initial conditions are carefully selected, the resulting decaying oscillatory response may be dominated by a single mode of vibration. The corresponding natural frequency can then be obtained trivially by counting zero crossings and damping can be estimated, for example, using the logarithmic decrement technique (Clough and Penzien, 2003). Otherwise, more sophisticated methods, such as the Ibrahim time domain method (Maia et al., 1997) may be required to extract the dynamic parameters of the structure. 


\subsubsection{Earthquake response tests}

Earthquake response tests consist of permanently installing sensors in the building whose dynamic properties are sought, waiting for relatively strong ground shaking to occur, and simultaneously measuring the ground shaking and the structure's corresponding response. Typically, the dynamic parameters are identified from transfer functions between the acceleration responses at upper levels and the measured accelerations at ground level (Goel and Chopra, 1997; Udwadia and Trifunac, 1974). For earthquake engineering purposes, dynamic properties identified from such tests provide invaluable data as they represent the dynamic properties of the structure during an actual ground shaking event. As mentioned previously, these properties have been shown to vary with the amplitude of the ground shaking (McVerry, 1979; Trifunac et al., 2001a). However, in areas of moderate seismicity, such tests may require considerable patience since the occurrence of significant earthquake shaking may be quite rare. Further, these tests require the permanent instrumentation of the building under study; therefore a large number of sensors are required to obtain reliable information on the spatial variation of the response (mode shapes) and on the response of different buildings.

\subsubsection{Ambient vibration tests}

Ambient vibration tests (AVT) rely on low-amplitude excitation from ambient sources, such as wind and micro-tremors, to drive building motion, which is measured and analyzed to obtain the vibration properties of the structure. In contrast to the methods described above, the input forces driving building motion are not known in ambient tests. Therefore, to extract the dynamic parameters of the structure, the excitation is assumed to be a broadband, stationary Gaussian white noise. This implies 
that the excitation has approximately equal energy content throughout the frequency range of interest (Bendat and Piersol, 2000).

Referring back to equation 2.33, under the assumption of a constant input spectrum at each input DOF, $\{F(\omega)\}$, the FRF matrix, $[H(\omega)]$ is directly related to the output spectra, $\{X(\omega)\}$. Since the FRF between any two DOF exhibits peaks at the natural frequencies of the various vibration modes of the structure, it follows that these frequencies can be identified directly from the output spectra, under the assumption of white noise input. This is the basis for all frequency domain modal identification techniques using ambient vibration data, as will be explained in more detail in section 3.2.2. It should be noted that the spectra of the unknown inputs are not likely to be constant in practice; however compared to the large variation of the FRF with frequency - particularly near a resonant frequency -, the input spectra are only weakly dependent on frequency (Brownjohn, 2003).

AVT have been widely touted as a practical modal identification tool, mainly due to the easy and inexpensive setup required to perform such tests, as well as the fact that the modal properties are obtained under the actual operating conditions of the structure. They have been shown to yield good estimates of the natural frequencies and mode shapes under normal operating conditions, but estimates of modal damping ratios are not as reliable (Brownjohn, 2003). This has generally been attributed to imperfect assumptions about the input forces (stationary white noise), leakage, or insufficient averaging in the estimation of spectral density (see section 2.5.3) (Rainieri et al., 2010).

The first known report of ambient vibration tests was a study of the fundamental periods of structures by the U.S. Coast and Geologic Survey (Carder, 1936; Ivanovic et al., 2000b). The method began to stimulate wide interest after the work of Crawford and Ward (1964), who sought to 
compare the experimental vibration periods of actual buildings with those predicted from mathematical models. They showed that ambient tests could be used to find the first few natural frequencies and corresponding mode shapes of a structure. Since then, a very large number of studies have been published on the subject. The following are some of the more important studies, as pertains to this particular research project, and are by no means an exhaustive list of references on the subject.

Over the years, ambient vibration tests have been conducted on a wide range of full-scale structures to obtain their dynamic parameters, including buildings (Beck et al., 1995; Ivanovic et al., 2000a), bridges (Brownjohn et al., 1999; Farrar and James III, 1997), and dams (Darbre et al., 2000). Also, many studies compared the dynamic characteristics obtained from ambient vibration tests with those obtained from other testing methods, and investigated the effects of excitation amplitude on the measured properties. By comparing the results of ambient and forced vibration tests on two buildings in California, Trifunac (1972) showed that the results of ambient and forced vibration tests agreed very well, but that slight reductions in frequencies could be expected in forced vibration tests when the excitation amplitudes (between the different testing methods) were significantly different. Similar findings were reported by other authors (Hans et al., 2005). Udwadia and Trifunac (1974) compared the results of ambient, forced and earthquake tests on two buildings and showed that the natural frequencies during moderate ground shaking were significantly lower than those from lower amplitude excitations (ambient and forced vibration tests and smaller earthquakes), but that the buildings seemed to recover some of their lost stiffness over time. This recovery appeared to be almost immediate in the case of small ground motions, but took longer in the case of stronger ground motions. This phenomenon was later attributed mainly to soil-structure interaction (Trifunac et al., 2001a; Trifunac et al., 2001b). Other studies explored the changes in system 
parameters before and after retrofit (Çelebi and Liu, 1998), during construction (Memari et al., 1999; Ventura and Schuster, 1996), due to non-structural elements (Pan et al., 2006), and due to water level in a dam reservoir (Proulx et al., 2001).

Another important aspect of ambient vibration research has been the development of algorithms to treat ambient vibration data. The development of the FFT algorithm (Cooley and Tukey, 1965) has given rise to many frequency domain techniques. The simplest and most common method, which is still used to some extent today, is known as the peak-picking method. It consists of plotting the spectral density curves of the measured responses and extracting the frequencies from peaks in these curves. The mode shapes are estimated by examining the relative magnitudes of the spectral density curves of the different measurement channels, and the damping by the half-power bandwidth method (Craig and Kurdila, 2006). In a series of papers, Akaike (1969a; 1969b) suggested first fitting an autoregressive (AR) model to the time series data to improve the spectral density estimates. AR-based methods began attracting increasing attention from then on (Gersch and Martinelli, 1979; Gersch et al., 1973; Kadakal and Yuzugullu, 1996). Many other algorithms have been introduced over the years using Hilbert-Huang transforms (Yang et al., 2003), neural networks (Huang et al., 2003), and subspace formulations (Van Overschee and De Moor, 1993), many of which have not achieved widespread popularity. The most popular algorithms currently used for system identification from ambient vibration data are the frequency domain decomposition (FDD) method (Brincker et al., 2001b) and the stochastic subspace identification (SSI) method (Van Overschee and De Moor, 1993). Both methods are quite robust, have been extensively validated, and have been used successfully by many researchers to process ambient vibration data (Gentile and Gallino, 2008; Lamarche et al., 2008; Structural Vibration Solutions A/S, 2010). In fact, 
these methods have been incorporated in specialized commercial software packages.

In this study, ambient vibration tests were performed in 39 buildings. Due to its simplicity, as compared to SSI, FDD was used to determine the dynamic parameters of these buildings. The details of the experimental methods are described in section 3.

\subsection{Stochastic processes}

Data obtained from ambient vibration tests are generally time histories of the displacement, velocity or acceleration response of various DOF. Each of these time histories can be considered as a realization of a stochastic (or random) process. This means that future values in the time signals cannot be accurately predicted and must instead be described by probabilities and statistical averages (Bendat and Piersol, 2000). Thus, analysis of ambient vibration data borrows heavily from the theory of random data analysis, the relevant aspects of which are discussed below. This discussion has mainly been adapted from Bendat and Piersol (2000) and Oppenheim and Schafer (1999).

\subsubsection{Mean and autocorrelation function}

Any single observation of a stochastic process represents one of many possible results. In other words, different observations of the same process are not likely to be identical. However, these processes can be characterized by certain statistical properties. For the discussion below, suppose an ensemble of $N$ observations of the same process, $x(t)$, have been obtained, yielding $N$ sample records, $x_{k}(t), k=1,2, \ldots N$. 
The two most common properties used to describe a stochastic process are its mean value and autocorrelation function, respectively defined as

$$
\begin{aligned}
& \mu_{x}=E[x(t)] \\
& R_{x x}(\tau)=E[x(t) x(t+\tau)]
\end{aligned}
$$

in which $E[$.$] indicates the expected value operation and \tau$ represents time lag, which can take on any real value. Note that the mean value is a single value, whereas the autocorrelation function is an infinite function defined over the domain of real numbers. It follows from equation 2.68 that the autocorrelation function of a periodic signal should exhibit peaks when the time lag is a multiple of the signal's period.

\subsubsection{Stationarity and ergodicity}

The above properties can be estimated by averaging across the ensemble at a particular instant in time $t_{1}$, as follows:

$$
\begin{aligned}
& \mu_{x}\left(t_{1}\right)=E\left[x\left(t_{1}\right)\right]=\frac{1}{N} \sum_{k=1}^{N} x_{k}\left(t_{1}\right) \\
& R_{x x}\left(t_{1}, \tau\right)=E\left[x\left(t_{1}\right) x\left(t_{1}+\tau\right)\right]=\frac{1}{N} \sum_{k=1}^{N} x_{k}\left(t_{1}\right) x_{k}\left(t_{1}+\tau\right)
\end{aligned}
$$

If the calculated mean and autocorrelation properties are the same, regardless of the time instant selected, the process is said to be stationary. Alternatively, these properties can be estimated by averaging across time in a single sample record: 


$$
\begin{aligned}
& \mu_{x}(k)=E\left[x_{k}(t)\right]=\lim _{T \rightarrow \infty} \frac{1}{T} \int_{0}^{T} x_{k}(t) d t \\
& R_{x x}(k, \tau)=E\left[x_{k}(t) x_{k}(t+\tau)\right]=\lim _{T \rightarrow \infty} \frac{1}{T} \int_{0}^{T} x_{k}(t) x_{k}(t+\tau) d t
\end{aligned}
$$

If the time-averaged properties are identical for all sample records, and are equal to the corresponding ensemble averages, the process is said to be ergodic. Since few - often only one - records are typically obtained for each DOF in ambient vibration tests, it is convenient to assume that the process is ergodic, such that the analysis can be performed using a single record for each DOF.

\subsubsection{Spectral density}

Another important property of a stochastic process is its spectral density. The spectral density of a time signal (or between two signals) is a measure of the energy content per unit frequency in that signal. It is thus a very useful tool in identifying the dominant frequencies in a particular signal. In fact, most frequency domain methods used to identify the dynamic properties of structures from ambient vibration tests make use of the spectral density.

The spectral density, $G_{x y}(\omega)$, between two time history records $x(t)$ and $y(t)$, having corresponding Fourier transforms $X(\omega)$ and $Y(\omega)$, is defined as

$$
G_{x y}(\omega)=E\left[X(\omega) Y(\omega)^{*}\right]
$$

where * denotes the complex conjugate. An initial estimate can be obtained by performing an FFT for each raw time signal to obtain $X(\omega)$ and $Y(\omega)$ and simply omitting the expected value operation. However, this 
estimate, known as the periodogram, has very large variance, and fluctuates significantly about the true spectrum. To improve the estimate, each record can be divided into a series of $r$ sub-records of shorter duration and the periodogram is computed for each one. An improved estimate of the spectral density is then obtained by averaging the periodograms of all sub-records, as follows:

$$
G_{x y}(\omega) \approx \frac{1}{r} \sum_{m=1}^{r} X^{m}(\omega) Y^{m}(\omega)^{*}
$$

In the above equation, $r$ represents the number of sub-records and $m$ is an index referring to a particular sub-record (not an exponent). This method of averaging periodograms, commonly referred to as Bartlett's method, significantly reduces the variance of the spectral density estimates.

However, Bartlett's method is still prone to error due to leakage, whereby power from a particular frequency leaks to neighboring frequencies due to the FFT operation. To avoid this, each time window is first multiplied by a leakage reduction window, yielding modified sub-records $\widetilde{x}^{m}(t)$ and $\widetilde{y}^{m}(t)$, and the FFT of each one is performed, yielding $\widetilde{X}^{m}(\omega)$ and $\widetilde{Y}^{m}(\omega)$, which can again be multiplied and averaged, as in equation 2.74 , to produce an improved estimate of spectral density. This method is known as the modified periodogram approach (or Welch's method) and the most common leakage reduction window is the Hanning window (Bendat and Piersol, 2000). This is the most common way of estimating the spectral density between two signals. The procedure is illustrated in Figure 2.4. Note that the sub-records shown in this figure do not overlap. In practice, it is common to use overlapping time windows to increase the number of periodograms in the averaging operation. 


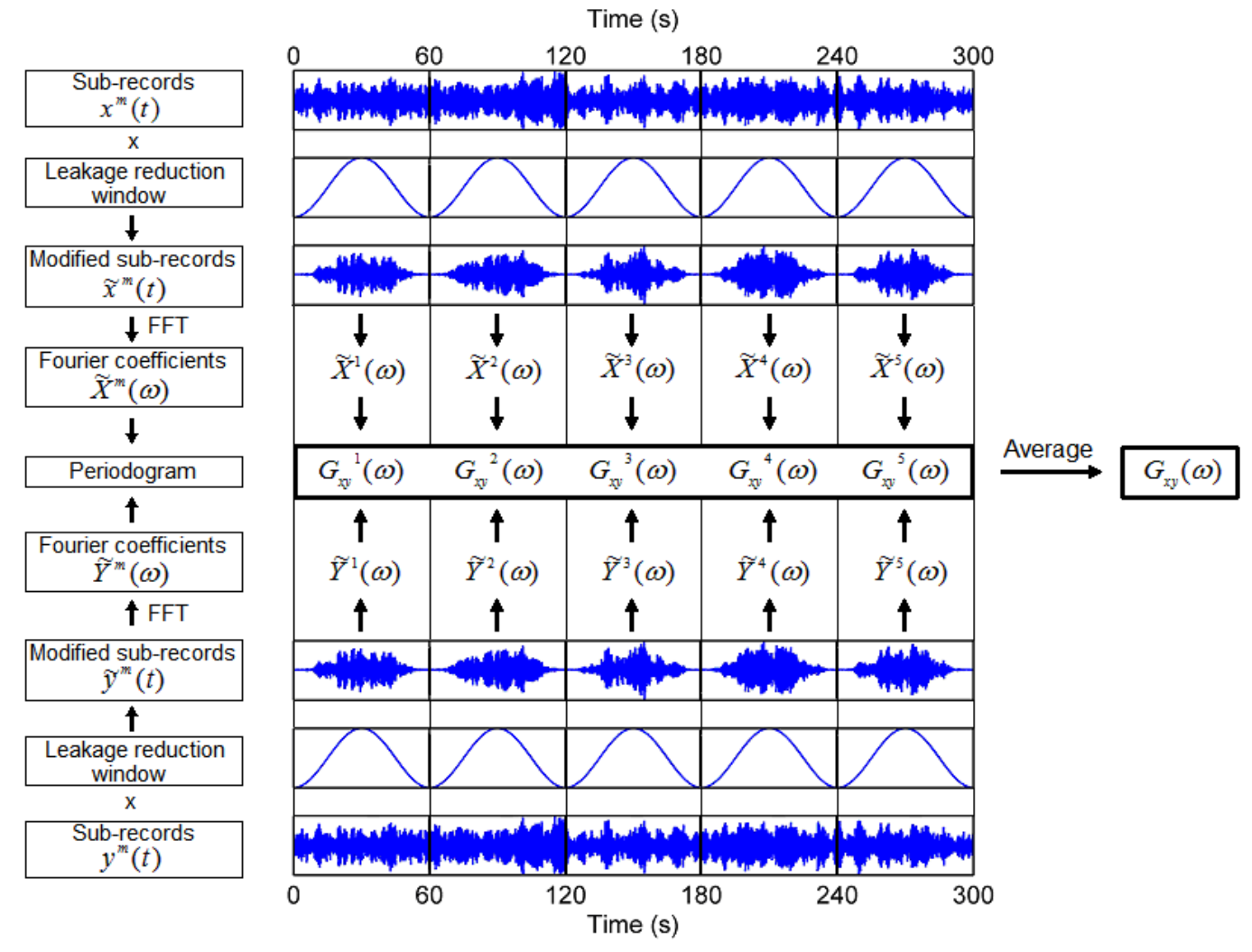

Figure 2.4: Spectral density estimation using Welch's method

Alternatively, the spectral density can be estimated by Fourier transforming the cross-correlation function. Indeed, the Weiner-Khintchine relations state that the spectral density of a signal and its correlation function are Fourier pairs (Bendat and Piersol, 2000). Similarly to the autocorrelation function, the cross-correlation function between signals $x(t)$ and $y(t)$ is defined as

$$
R_{x y}(\tau)=E[x(t) y(t+\tau)]
$$

This method of spectral density estimation is however not commonly used. In fact, it is more common to perform an inverse Fourier transform of the spectral density estimated by Welch's method to estimate the crosscorrelation function (Bendat and Piersol, 2000). The Weiner-Khintchine 
relations will be important to understanding the enhanced frequency domain decomposition method described in section 3.2.2.3. 


\section{Experimental methods}

Between June 2007 and August 2009, ambient vibration tests were performed in 39 buildings in Montréal. In 27 of these, reinforced concrete shear walls (RCSW) provided the main resistance to lateral loads. The initial objective of this research was to explore the dynamic properties of buildings having different types of lateral load-resisting systems; however, since most of the buildings tested were categorized as RCSW buildings, it was decided to narrow the focus to these types of buildings alone. Velocities resulting from ambient excitations were recorded at several locations in each building, and the recorded motions were analyzed using enhanced frequency domain decomposition (EFDD) to obtain the dynamic properties of up to six vibration modes in the low frequency range. A detailed summary of the dynamic properties of each of the 39 buildings is available in a separate report (Gilles, 2010), and the detailed identification of the first two vibration modes of one of these buildings is presented in Appendix B. Let us however describe the experimental methods used to collect and analyze the data in order to extract the dynamic properties.

\subsection{Data collection}

\subsubsection{Equipment}

For each building, translational velocities resulting from ambient excitations were measured at several locations, distributed along both the height and plan dimensions, using two Lennartz LE-3D/5s triaxial seismometers. These sensors measure minute velocities in three orthogonal directions: two in the horizontal plane and one in the vertical 
direction. Table 3.1 shows some of the manufacturer's specifications for this type of sensor.

Table 3.1: Technical specifications of Lennartz LE-3D/5s sensors

\begin{tabular}{|c|c|}
\hline Eigenperiod & $5 \mathrm{sec}$ \\
\hline Bandwidth & $0.2-40 \mathrm{~Hz}$ \\
\hline Temp range & -15 to $60 \mathrm{deg} \mathrm{C}$ \\
\hline Sensitivity & $400 \mathrm{~V} /(\mathrm{m} / \mathrm{s})$ \\
\hline RMS noise at $1 \mathrm{~Hz}$ & $<1 \mathrm{~nm} / \mathrm{s}$ \\
\hline
\end{tabular}

The long eigenperiod makes this sensor suitable for dynamic tests in tall buildings, which are expected to have relatively long fundamental periods (as long as $4.35 \mathrm{~s}$ for the tallest building in this study). This sensor has a flat frequency response function between 0.2 and $40 \mathrm{~Hz}$, thus allowing the identification of resonant frequencies as low as $0.2 \mathrm{~Hz}$, which is lower than the fundamental frequency of most buildings, even very tall and flexible ones, and as high as $40 \mathrm{~Hz}$, which is well beyond the frequency of any vibration mode that is likely to significantly participate in seismic building response. Further, this sensor has high sensitivity: the value of 400 $\mathrm{V} /(\mathrm{m} / \mathrm{s})$ indicates that when the sensor perceives a $1 \mathrm{~m} / \mathrm{s}$ velocity, it outputs a $400 \mathrm{~V}$ signal to the data acquisition system. This high sensitivity, as well as the sensor's low noise level, is necessary for ambient vibration studies as the vibration amplitudes can be quite small. Typical velocities in this study were in the range of $10^{-5}$ to $10^{-7} \mathrm{~m} / \mathrm{s}$.

Each sensor was wired to an independent LEAS CitySharkll data acquisition system (DAS), as shown in Figure 3.1. These data acquisition units allow the users to set various measurement parameters, such as record length, sampling rate, and gain. Note that the gain refers to the amplification of the signal by an internal amplifier in the data acquisition system prior to recording the data. A large gain therefore increases the strength of a signal. The data were then stored on compact flash cards. Other components required to carry out the measurements included a 
remote control starter (RCS) and radio antennae, GPS receivers, connecting cables, compact flash cards, and walkie-talkies.

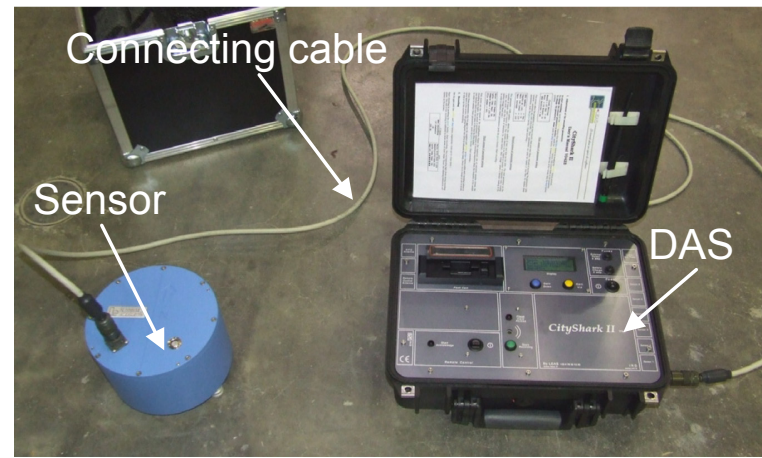

(a)

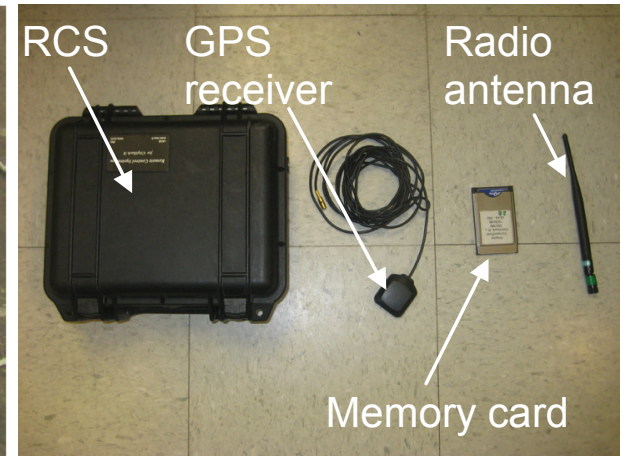

(b)

Figure 3.1: (a) Typical setup of sensor/DAS pair; (b) Other equipment for ambient vibration tests

\subsubsection{Selection of measurement points}

Before performing ambient vibration measurements in a building, plans of the building were consulted to select the locations where the response would be measured. The selection of these points was based mainly on the need for good spatial distribution of the measurement points, both vertically and horizontally, such that the estimated mode shapes would be easy to interpret. However the choice was often significantly constrained by access restrictions. Whenever possible, measurements were taken on at least six floors, and in most buildings, measurements were taken at three locations on each floor, along a principal axis of rigidity, to permit the identification of both translation and torsion modes. For practical purposes, the principal axis of rigidity was generally approximated using an axis of symmetry of the building plan, as shown for example in Figure 3.2. In certain cases however, different measurement configurations were adopted, due for instance to access restrictions. 


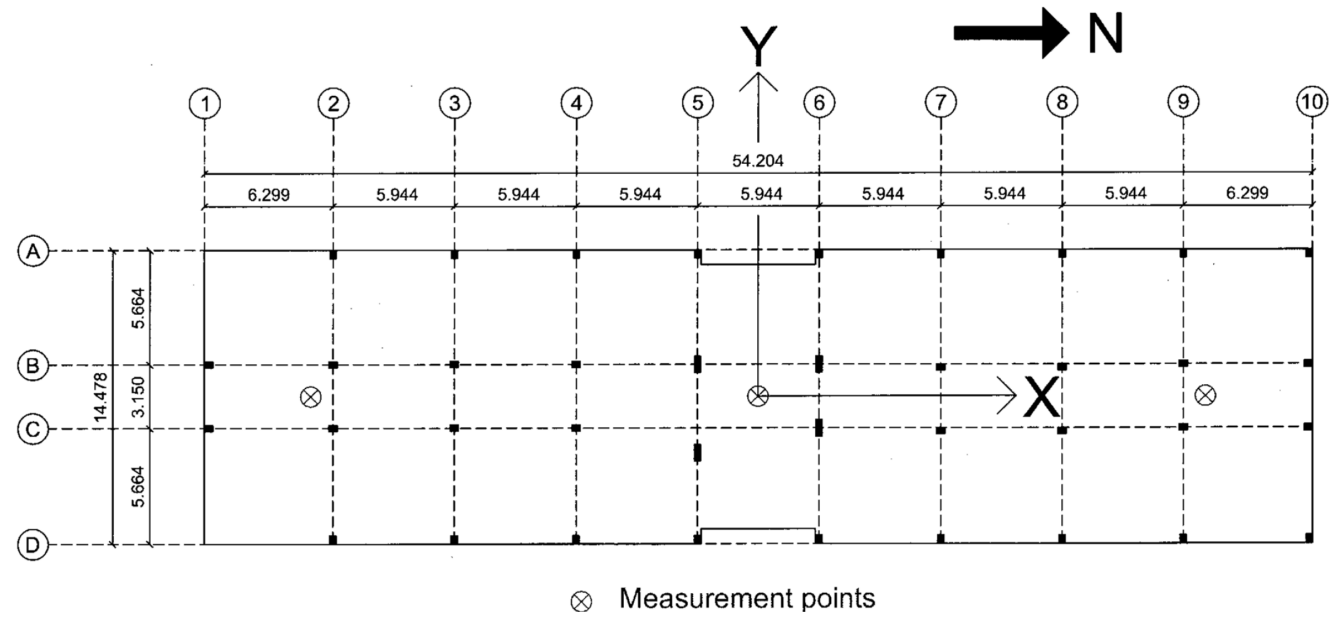

Figure 3.2: Typical selection of measurement points

\subsubsection{Test procedure}

Since only two sensors were available to carry out this research, measurements were carried out following a roving sensor approach. One of the two sensors - the reference sensor - was placed at a location in the structure where most of the vibration modes of interest were expected to participate in the response and where the measured response was expected to be largest. Typically, the reference sensor was placed on one of the upper floors, away from the centre of rigidity of the building. The remaining sensor - the roving sensor - was moved to the different points in the building that were selected for measurement. For the first measurement setup, the two sensors were typically placed at the reference location. In this way, the data from the reference sensor were only used to scale the data from different measurement setups, as will be discussed in section 3.2.2.2.

As mentioned above, in each measurement setup, the two sensors were connected to separate data acquisition systems, from which the measurement parameters were set. The most important parameters were the record length, sampling frequency, and gain. In general, five minute 
data records were taken at a sampling frequency of $1000 \mathrm{~Hz}$ for both data acquisition systems and for each measurement setup. This record length was selected based on prior experience with the equipment, as well as practical considerations concerning the overall time required to perform vibration tests in a building. We will see in section 4 that modal properties were reliably extracted for all buildings with this record length. In regard to the sampling frequency, it was not necessary to sample at such a high frequency: since we were typically only interested in frequencies below 25 $\mathrm{Hz}$, by virtue of the Nyquist sampling theorem (Oppenheim and Schafer, 1999), we could have used a sampling frequency as low as $50 \mathrm{~Hz}$. However, we sampled at a very high frequency for synchronization purposes, as will be shown in section 3.2.1.2, and we down-sampled during data processing to reduce computation time. Finally, the gains for the two data acquisition systems were initially set to the same value since the sensors were placed at the same location. This selection was based on a visual inspection of the maximum signal obtained from the three channels on a graphic display on the data acquisition systems. The idea was to have as strong a signal as possible while limiting the amount of overflow, or clipped samples. As the building measurements progressed, the gain for the roving station was occasionally changed to reflect the changing vibration conditions at the different locations.

\subsubsection{Synchronization}

One of the main challenges with this measurement system was that the two sensors were connected to independent data acquisition systems (DAS). Therefore, significant efforts were required to ensure that the data from the two systems were synchronous. Without synchronous data, the mode shapes could not have been precisely extracted, as it would have been difficult (though not impossible) to infer the relative phases of the different points in the building for a given vibration mode. This problem 
was occasionally circumvented by using the remote control starter (RCS) to start the two data acquisition systems simultaneously for all measurements in a given building. However, in most cases, the RCS did not have sufficient range to be used for all vibration measurements within a building. The following procedure was implemented in each building vibration test to ensure that the two systems were synchronous throughout the measurements.

Prior to the first vibration record, the internal clocks of the two DAS were synchronized using GPS receivers. A dummy data record was then taken using the RCS, which would later be examined to ensure that the two systems were initially synchronous. Then, the measurements were carried out using the roving sensor approach explained above. Whenever possible, the two data acquisition systems were started with the RCS; but when that was not possible, they were started manually at approximately the same time by two operators who communicated via walkie-talkie. After all recordings were complete, another dummy data record was taken using the RCS. For each dummy data record (before and after the building measurements), the starting time stamps, which are recorded in the raw data file, for each DAS were compared. If the start times were sufficiently close (within a few milliseconds), the DAS were deemed to be synchronized at the beginning and end of the building vibration test, and thus throughout the data records.

To synchronize the records for which the two DAS were started manually, the starting time stamps in the data records were used. Since the systems' internal clocks were synchronized, any difference in the starting time stamps indicated that the data records were not started at exactly the same time by the two operators. To correct this, the difference in the time stamps of the reference and roving stations were computed for each measurement setup. The data records were then truncated, such that only 
the synchronous portions of the data records were used in the analysis. This is explained in more detail in section 3.2.1.2.

\subsection{Data analysis}

To obtain the dynamic properties of each building, the measured ambient vibration data were analyzed using the enhanced frequency domain decomposition (EFDD) method, as implemented in the commercial software ARTeMIS Extractor ${ }^{\text {TM }}$ (Structural Vibration Solutions A/S, 2009a). However, the ambient vibration data were not immediately amenable to data analysis: certain pre-processing steps were required. The steps involved in data pre-processing and EFDD are briefly summarized below.

\subsubsection{Data pre-processing}

\subsubsection{Converting data to velocity}

The raw data obtained from every measurement, for each data acquisition system, were stored on a compact flash card as a text file. Each text file contained a header, listing the measurement parameters, followed by three columns of data corresponding to the time histories of the Up-Down, North-South, and East-West directions, as shown in Figure 3.3. Note that the Up-Down data were ignored since this study focused on lateral building vibrations. Note also that the North-South direction, which is indicated on the sensors, was selected prior to each building test and the same orientation was maintained for all measurements, as shown in Figure 3.2. 


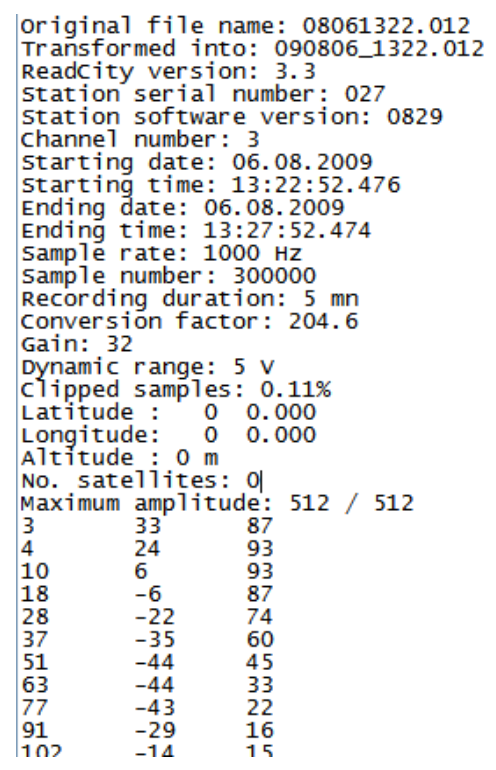

Figure 3.3: Excerpt of a typical data file from CityShark II data acquisition system

The raw data had units of bits. To convert the data to velocity, the gain and conversion factor, listed in the header, were used to calculate the input voltage to the CitySharkll data acquisition system from the sensors, as per the manufacturer's specifications:

$$
V_{\text {in }}=\frac{\text { Data }}{\text { Gain } \cdot C F}
$$

where $C F$ was the conversion factor, and $V_{i n}$ had units of volts $(\mathrm{V})$. Then the sensitivity of the sensor, $400 \mathrm{~V} /(\mathrm{m} / \mathrm{s})$, was used to convert the voltage output from the sensor (or voltage input to the data acquisition system) to units of velocity, as follows:

$$
v=\frac{V_{\text {in }}}{400}=\frac{\text { Data }}{\text { Gain } \cdot C F \cdot 400}
$$

where $v$ is expressed in $\mathrm{m} / \mathrm{s}$. 


\subsubsection{Synchronizing data}

As mentioned above, some of the data records for the reference and roving sensors were started using the RCS, while others were started manually. The reference and roving records started using the RCS were already synchronous, and therefore the entire data records were used directly without any further pre-processing. On the other hand, the records that were started manually were only approximately synchronous, insofar as the records were started at approximately the same time by the operators of the two stations. These therefore needed to be truncated to keep only the portions of data from each record that were exactly synchronous, leading to records that were slightly shorter than five minutes. This was done using the start time stamps in the data records.

Suppose the start times for the reference and roving stations were respectively $t_{\text {ref }}$ and $t_{\text {rov }}$. The corresponding end times were therefore $t_{\text {ref }}+$ $5 \mathrm{~min}$ and $t_{\text {rov }}+5 \mathrm{~min}$. Suppose now that the reference station was started slightly before the roving station $\left(t_{\text {ref }}<t_{\text {rov }}\right)$. This situation is illustrated in Figure 3.4, which shows that the synchronous data from the two stations lies between $t_{\text {rov }}$ and $t_{\text {ref }}+5 \mathrm{~min}$. The difference between the time stamps $\left(t_{\text {rov }}-t_{\text {ref }}\right)$ represents the amount of time that needs to be truncated from each data record. In such cases, the beginning of the reference record and the end of the roving record were truncated before the analysis was performed, and the truncated data records were shorter than five minutes by exactly the difference between the start times. In contrast, when the roving station was started before the reference station, the beginning of the roving record and the end of the reference record were truncated. 


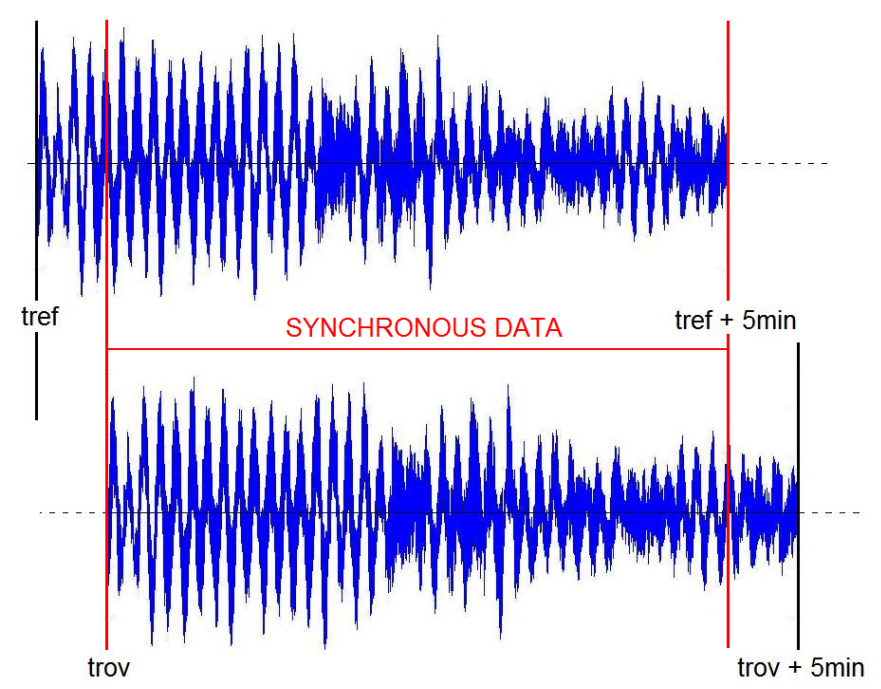

Figure 3.4: Truncation process for data synchronization

Since the data were sampled at discrete time intervals, dictated by the selected sampling frequency, the number of data points to truncate was calculated using the sampling frequency, $f_{s}$, as

$$
\text { \#Data Pts }=\left|t_{\text {ref }}-t_{\text {rov }}\right| f_{s}
$$

The start time stamps in the data files were quoted to the third decimal (milliseconds). Therefore, by selecting a sampling frequency of 1000 samples per second $(1000 \mathrm{~Hz})$, the number of data points to truncate was always an integer, making the truncation process exact. This explains why such a high sampling frequency was used.

The above pre-processing steps were performed using a MATLAB® (The Mathworks, 2008) routine, the output of which was a text file ready for use in ARTeMIS. Each such file included simply four columns of data corresponding to the North-South and East-West velocity time histories of 
the reference and roving locations (recall that the Up-Down data were neglected).

\subsubsection{Down-sampling data}

Due to the synchronization procedure, the data were acquired at a sampling rate much higher than that required for the purposes of extracting the modal parameters. This resulted in excess data, which would have needlessly increased the time required to perform data analysis. To avoid this, the data were down-sampled (or decimated) prior to analysis. The decimation process essentially involves re-sampling the data at a reduced sampling rate by keeping data points at evenly spaced times, while discarding the remaining data points. However, to avoid aliasing errors, the data are first low-pass filtered to remove the contribution of high frequencies to the original signal. Many types of antialiasing filters can be used, and the reader may refer to Oppenheim and Schafer (1999) for more information on filtering.

In this study, decimation was performed directly in ARTeMIS prior to signal processing. This software uses an 8th order Chebyshev Type 1 low-pass filter (Structural Vibration Solutions A/S, 2009b). Usually, a $20^{\text {th }}$ order decimation was performed - that is, every $20^{\text {th }}$ data point was kept resulting in an effective sampling rate of $50 \mathrm{~Hz}$, and a Nyquist frequency of $25 \mathrm{~Hz}$. In this way, the search for modal frequencies was limited to frequencies between 0 and $25 \mathrm{~Hz}$. 


\subsubsection{Estimation of modal parameters}

To explain the data processing procedure, let us first discuss the theory behind the enhanced frequency domain decomposition method (EFDD). The implementation of EFDD in ARTeMIS will then be explained.

For the following discussion, suppose ambient vibrations were simultaneously recorded at $N$ degrees-of-freedom (DOF), giving rise to a set of velocity time history records $x_{i}(t), i=1,2, \ldots, N$. We will see that nonsynchronous data collection involves a few extra steps to identify the modal properties. For simplicity, Figure 3.5 shows these DOF as a set of collinear points, even though measurements were typically distributed along both the vertical and horizontal dimensions to permit identification of both torsion and translation modes.

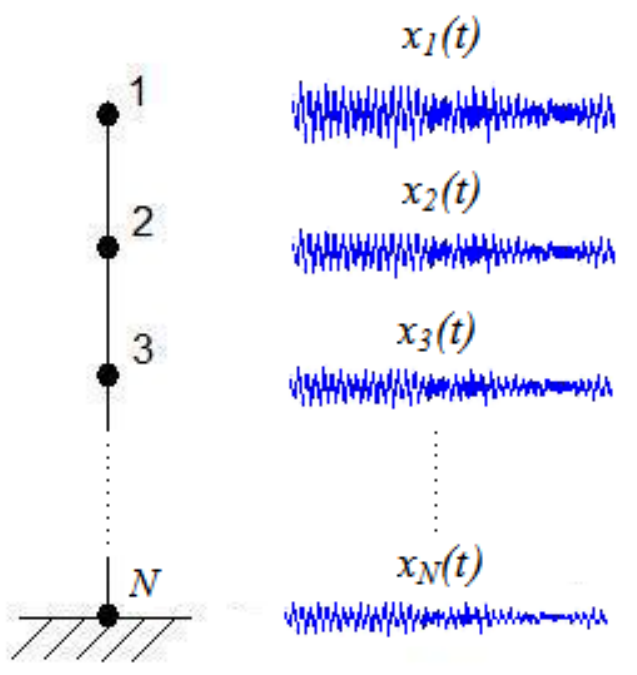

Figure 3.5: Example of time history records collected at $\mathbf{N}$ collinear DOF

\subsubsection{Spectral density estimation}

As mentioned previously, spectral density is a measure of a signal's energy content per unit frequency. Spectral density is thus a useful tool in 
identifying the frequencies that contribute the most energy to a particular signal. For this reason, spectral density estimation forms the backbone of most frequency domain modal identification methods, including EFDD.

The first step of EFDD is to estimate the spectral density functions between all the different response signals (records). Assuming each data record to be a realization of an ergodic process, the spectral densities can be obtained using a single record for each location. To obtain good estimates of the spectral density functions, each of the $N$ records, $x_{i}(t)$, are first divided into $r$ sub-records, $x_{i}^{m}(t)$, where $m=1,2, \ldots, r$, as explained in section 2.5.3. Here $m$ is a superscript indicating the sub-record, and not an exponent. These sub-records typically overlap. Note that the length of the sub-records dictates the frequency spacing resulting from the FFT operation. However, in ARTeMIS, the length of the sub-records is chosen indirectly by specifying the number of frequency lines between 0 and the Nyquist frequency. Typically, 1024 frequency lines and a Nyquist frequency of $25 \mathrm{~Hz}$ were selected, yielding a frequency spacing of 0.02441 $\mathrm{Hz}$. This means that each sub-record typically had a length of $40.967 \mathrm{~s}$.

Each sub-record is multiplied by a Hanning window to reduce the effects of leakage, generating a set of modified sub-records $\widetilde{x}_{i}{ }^{m}(t)$. Then, an FFT is performed for each modified sub-record, yielding $\widetilde{X}_{i}^{m}(\omega)$. The power spectral density (PSD) matrix for each sub-record $m$ is then computed at each frequency by multiplying the appropriate Fourier coefficients. For example, the entry in row $j$ and column $k$, representing the spectral density between the records at DOF $j$ and $k$, for sub-record $m$, and frequency $\omega$ is

$$
G_{j k}^{m}(\omega)=\widetilde{X}_{j}^{m}(\omega) \cdot \widetilde{X}_{k}^{m}(\omega)^{*}
$$


where * indicates the complex conjugate. Each element of the PSD matrix is then obtained at each frequency by averaging across all $r$ sub-records:

$$
G_{j k}(\omega)=\frac{1}{N} \sum_{m=1}^{r} G_{j k}^{m}(\omega)
$$

Figure 3.6 shows the PSD matrices at discrete frequencies for a six-DOF system. Since there are six DOF, each matrix is $6 \times 6$. The colour within each cell (representing a matrix element) is used as an indicator of the magnitude of the spectral density between the records corresponding to the cell's row and column. Note that each PSD matrix (i.e. at each frequency) is Hermitian, which means that the entries on opposite sides of the main diagonal are complex conjugates, which follows from equation 3.4. Further, the elements along the main diagonal are real numbers, while the off-diagonal terms are generally complex.

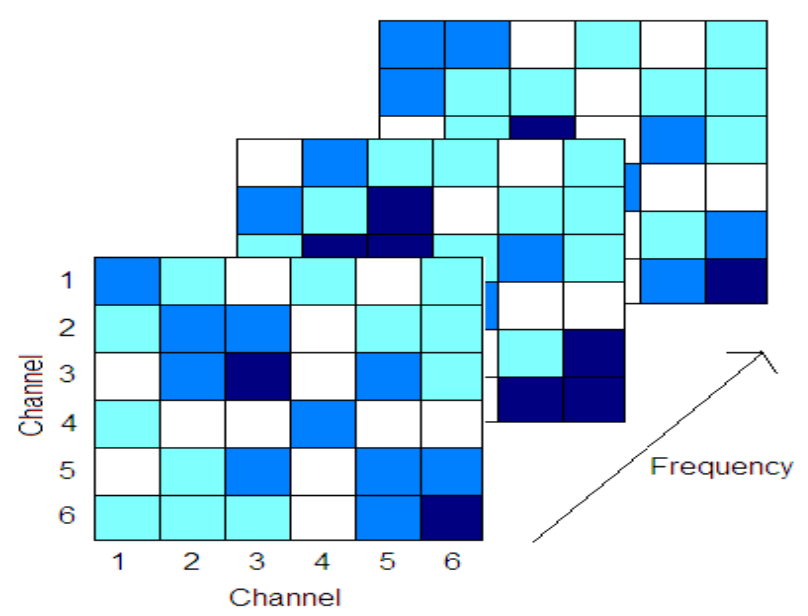

Figure 3.6: Illustration of PSD matrices at discrete frequencies for a 6-DOF system

Note that the units of spectral density are the square of the units of the original signals per unit frequency. In the present case, the original signals were velocity time histories (in $\mathrm{m} / \mathrm{s}$ ); hence, the spectral density between two signals had units of $\left[(\mathrm{m} / \mathrm{s})^{2} / \mathrm{Hz}\right]$. However, it is common to quote 
spectral density in decibels $(\mathrm{dB})$ relative to a reference quantity. For instance, using a reference quantity of $(1 \mathrm{~m} / \mathrm{s})^{2} / \mathrm{Hz}$, the spectral density in $\mathrm{dB}$ is computed as

$$
G[d B]=10 \log _{10}\left(\frac{G\left[(\mathrm{~m} / \mathrm{s})^{2} / H z\right]}{1(\mathrm{~m} / \mathrm{s})^{2} / H z}\right)
$$

One of the simplest frequency domain modal identification methods, known as the peak-picking method, involves plotting each spectral density function by considering one element of the PSD matrix over the frequency range of interest (Brownjohn, 2003). The peaks are identified as resonant frequencies and the mode shapes are inferred by examining the relative magnitudes (and phases) of the spectral densities of the different signals, contained in the PSD matrix, at each identified resonant frequency. Modal damping ratios can be estimated by the half-power bandwidth method (Clough and Penzien, 2003) on any of the spectral density plots.

Note that identification of modal parameters from the spectral densities of the measured motions, without any consideration of the excitation sources causing these motions, is only valid if the excitation is a broadband, stationary, Gaussian white noise. That is, the spectrum of the excitation must be flat over the frequency range of interest. Recall from equation 2.33 that the inputs and responses of a structure are related through the structure's FRF matrix, which contains information about its dynamic properties. Considering also equation 3.4, it follows that the input spectral density matrix, $\left[G_{f f}(\omega)\right]$, and output spectral density matrix, $\left[G_{x x}(\omega)\right]$, are related through the FRF matrix, $[H(\omega)]$, as follows:

$$
\left[G_{x x}(\omega)\right]=[H(\omega)]\left[G_{f f}(\omega)\right][H(\omega)]^{H}
$$


where ${ }^{H}$ denotes the Hermitian transformation (complex conjugate transpose). Hence, assuming each entry of the input spectral density matrix to be constant across the frequency range of interest, the output PSD matrix is related directly to the FRF matrix of the structure and the dynamic properties can be estimated directly from the output PSD matrix. Though this assumption is likely not strictly true in general, the peakpicking technique has nevertheless been shown to yield adequate estimates of frequencies and mode shapes, as discussed previously. However it is difficult to identify closely-spaced modes (Brincker et al., 2001b). Frequency domain decomposition is essentially an extension of this method, which keeps much of its simplicity while improving on some of its deficiencies.

\subsubsection{Frequency domain decomposition (FDD)}

Data recorded simultaneously for all measurement points

Rather than plotting the spectral densities directly, singular value decomposition (SVD) of the PSD matrices is first performed. In the following, it is understood that we are working with the output PSD matrices. Therefore, the subscript $x x$ - used to differentiate the output spectral density matrix, $\left[G_{x x}(\omega)\right]$ from the input sectral density matrix, $\left[G_{f f}(\omega)\right]$ - is dropped from the notation. The SVD of a square matrix transforms it into a set of three matrices of the same size in the following way (Schott, 2005):

$$
[G]=[U][S][V]^{H}
$$


where $[G]$ is the matrix to be decomposed (in this case, the output PSD matrix at each frequency), $[S]$ is the diagonal singular value matrix, $[U]$ and $[V]$ are unitary matrices containing the orthonormal left- and right- singular vectors, respectively, and ${ }^{H}$ denotes the Hermitian transformation. The singular values are listed in descending order along the main diagonal of $[S]$ and are always real, non-negative quantities. On the other hand, the singular vectors are generally comprised of complex quantities.

Since the PSD matrix at each frequency $[G(\omega)]$ is Hermitian, its SVD degenerates into the spectral decomposition, in which the matrices $[U(\omega)]$ and $[V(\omega)]$ are identical. This leads to the following equation:

$$
[G]=[U][S][U]^{H}=\left[\left\{u_{1}\right\}\left\{u_{2}\right\} \cdots\left\{u_{N}\right\}\right]\left[\begin{array}{cccc}
S_{1} & 0 & \cdots & 0 \\
0 & S_{2} & \cdots & 0 \\
\vdots & \vdots & \ddots & \vdots \\
0 & 0 & \cdots & s_{N}
\end{array}\right]\left[\begin{array}{c}
\left\{u_{1}\right\}^{H} \\
\left\{u_{2}\right\}^{H} \\
\vdots \\
\left\{u_{N}\right\}^{H}
\end{array}\right]
$$

in which the frequency $\omega$ is dropped to ease the presentation. But note that this decomposition is performed separately at each frequency. Interestingly, the singular vectors then are the orthonormal eigenvectors of $[G]$, and the singular values are the corresponding eigenvalues (Schott, 2005). Therefore, the singular vectors represent an estimate of the system's mode shapes, and the corresponding singular values provide an estimate of the contribution of each mode to the overall energy at each frequency. In other words, the SVD of the output PSD matrix is an approximation to its modal decomposition (Brincker et al., 2001b). 
Singular value decomposition is carried out for each PSD matrix - that is, at each discrete frequency resulting from the FFT operation. In practice, the first few singular values are plotted against frequency. Figure 3.7 shows the singular value plot of a building with well-separated modes. Four singular values are shown, but the first five resonant frequencies, $\omega_{p}$, can be identified from distinct peaks in the first singular value (upper black line) alone. At each of these resonant frequencies, the first singular vector $\left\{u_{1}\left(\omega_{p}\right)\right\}$ provides an estimate of the associated mode shape.

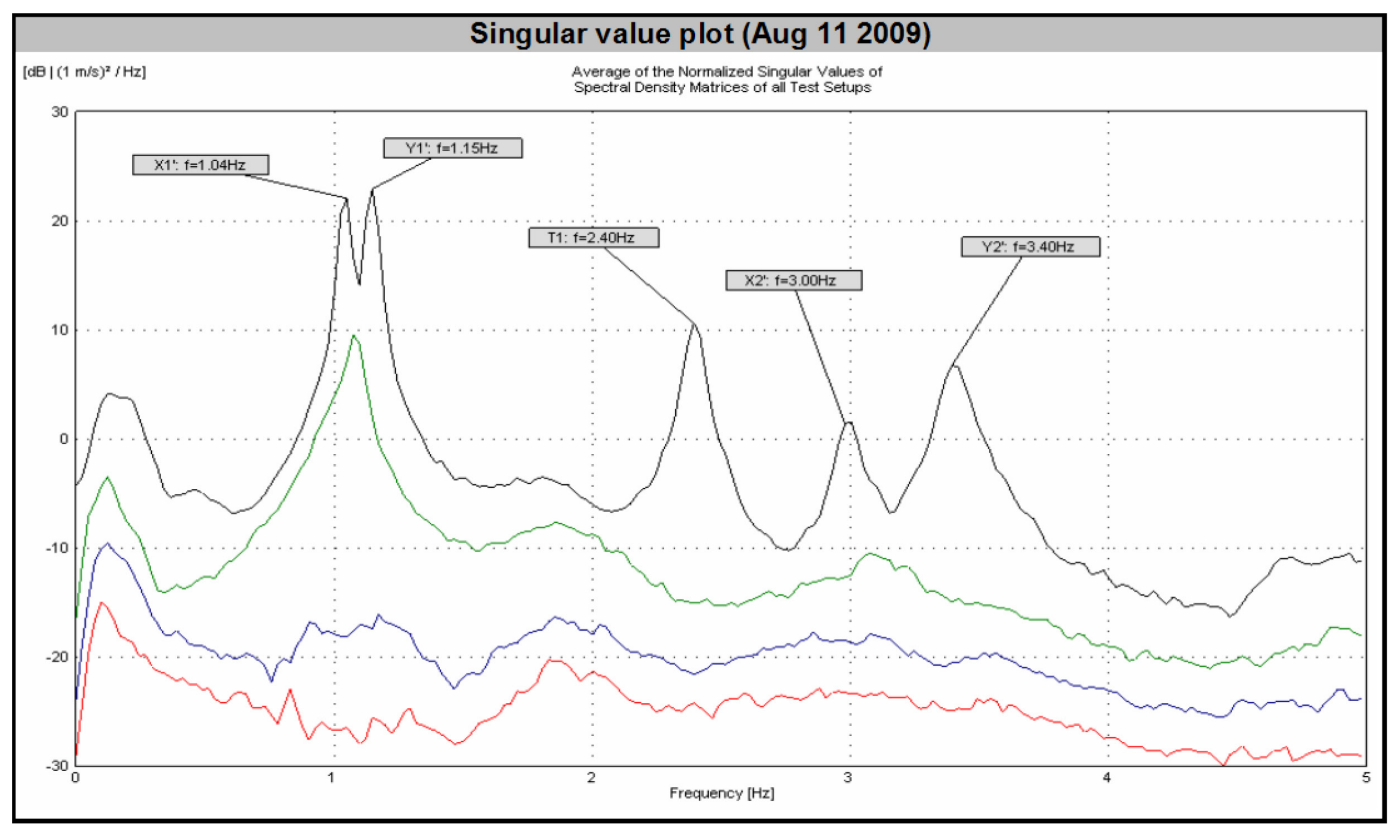

Figure 3.7: Singular value plot with well-separated modes

When two modes have close natural frequencies, both modes are likely to have significant energy at any frequency near their respective peaks. Thus, both the first and second singular values are likely to be large. To identify closely-spaced modes, it is therefore necessary to consider at least the second singular value in addition to the first. Since the first singular value is always larger than the second, the higher frequency peak in the first singular value is a continuation of the second singular value line at lower frequencies. This is illustrated in Figure 3.8, which corresponds to 
a building having two closely-spaced modes at frequencies of 3.10 and $3.14 \mathrm{~Hz}$. These modes could not have been dissociated using the peakpicking method.

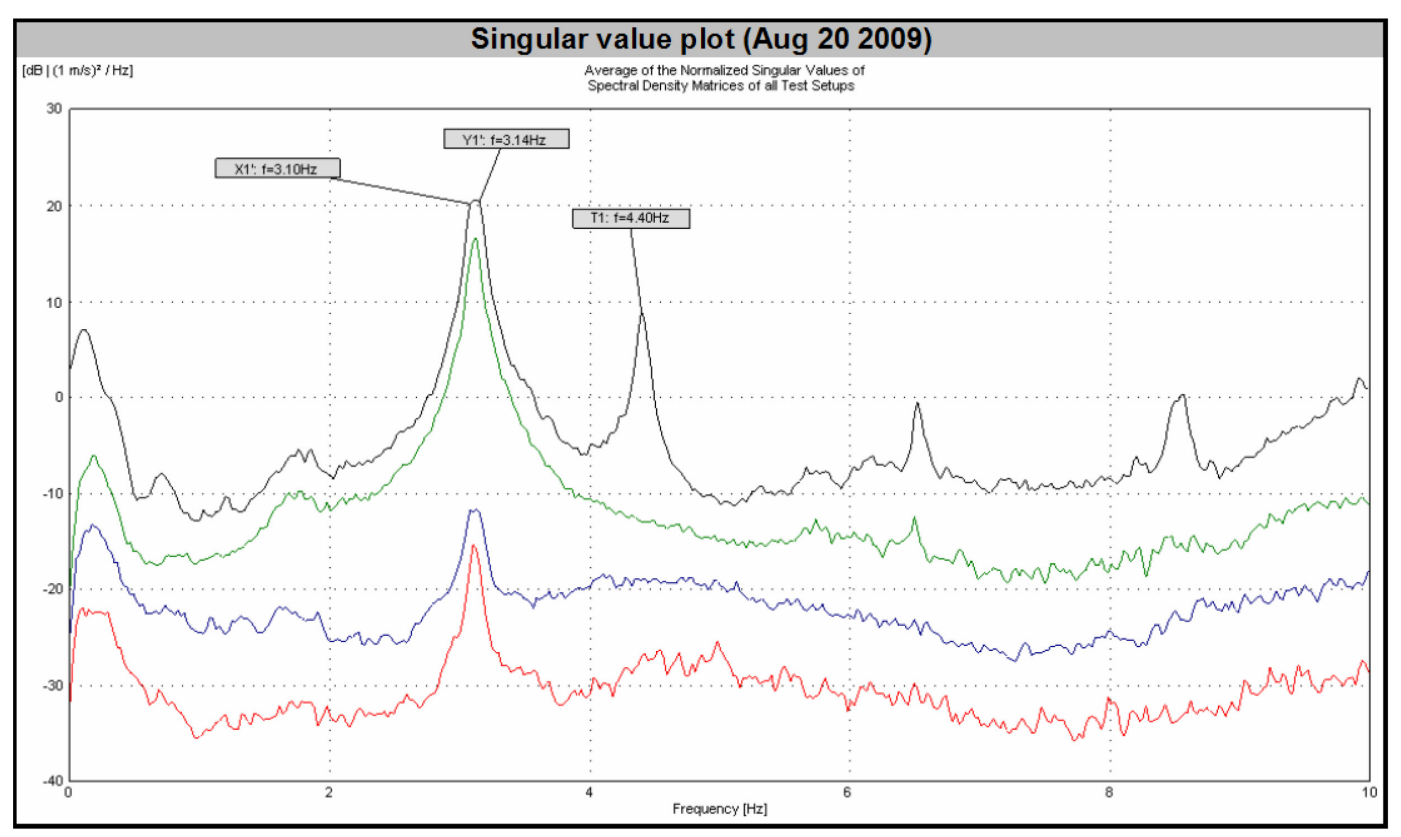

Figure 3.8: Singular value plot with closely-spaced modes

Data for all measurement points not obtained simultaneously

The above method assumes that the records for all measurement points were obtained simultaneously. In practice, this is rarely possible, and the analysis requires a few additional steps. Let us now consider the data obtained for the various buildings in this study.

For each measurement setup, four velocity time histories are considered, corresponding to the two horizontal directions for each of the reference and roving sensors. Thus, each setup involves the two reference degrees of freedom, which remain the same in all setups, and two roving degrees of freedom, which differ from one setup to the next. Suppose that a total of $n$ measurement setups were required for a particular building test. 
For each setup $q$, the $4 \times 4$ spectral density matrices, $[G(\omega, q)]$, are assembled at each frequency $\omega$, and singular value decomposition is performed, yielding a set of four singular values, $s_{i}(\omega, q)$, and four $4 \times 1$ singular vectors, $\left\{u_{i}(\omega, q)\right\}$, at each frequency (for each setup). At each frequency, the singular values from each setup are then averaged across the $n$ different setups. This averaging operation is done separately for each singular value (first, second, third, and fourth).

$$
s_{i}(\omega)=\frac{1}{n} \sum_{q=1}^{n} s_{i}(\omega, q), \quad i=1,2,3,4
$$

Note that the singular values in each setup are normalized by the area under the first singular value curve for that setup, before the averaging operation, to allow the identification of vibration modes that are only weakly excited in certain setups (Structural Vibration Solutions A/S, 2009b). The average normalized singular values are then plotted against frequency in a single display, as in Figures 3.7 and 3.8, and the potential modal frequencies are estimated from the peaks.

Since each setup involves different roving DOF, the mode shape estimate (involving all DOF) for each identified frequency is then assembled from the corresponding singular vectors from each setup. Considering the measurement procedure adopted in this study, there are two reference degrees of freedom, and each measurement setup involves two new degrees of freedom. If $n$ setups were required, it follows that the mode shapes involve $(2 n+2)$ DOF. The components of the mode shape at each frequency are obtained by considering the ratio of the components of the singular vectors for the roving DOF to those for the corresponding reference DOF (Brincker and Andersen, 1999). This must be done for the singular vectors of each setup to obtain a global mode shape estimate. 


\subsubsection{Enhanced frequency domain decomposition (EFDD)}

Frequency domain decomposition can be used to obtain estimates of resonant frequencies and the corresponding mode shapes. However, the accuracy of the modal estimation hinges on the accurate selection of the peaks in the singular value plot. Poor peak-picking, or overly large frequency spacing in this plot, can lead to inaccurate estimates. To improve these, as well as to estimate modal damping ratios and the uncertainty in both frequency and damping estimates, the enhanced frequency domain decomposition (EFDD) can be used.

Let us first introduce the modal assurance criterion (MAC), which is a measure of the correlation between two mode shape estimates $\left\{\phi_{l}\right\}$ and $\left\{\phi_{2}\right\}$, and is given by

$$
\operatorname{MAC}\left(\left\{\phi_{1}\right\},\left\{\phi_{2}\right\}\right)=\frac{\left|\left\{\phi_{1}\right\}^{H} \cdot\left\{\phi_{2}\right\}\right|^{2}}{\left|\left\{\phi_{1}\right\}^{H} \cdot\left\{\phi_{1}\right\}\right| \cdot\left|\left\{\phi_{2}\right\}^{H} \cdot\left\{\phi_{2}\right\}\right|}
$$

The MAC can be used to compare mode shape estimates from different data analysis methods, to compare mode shapes from experiments versus analytical models, to check the orthogonality of different modes within the same system, or finally to compare mode shapes for the same mode estimated at slightly different frequencies.

\section{Identification of SDOF bell}

Starting from the average normalized singular value plot (see Figures 3.7 and 3.8), the peaks are identified as potential modal frequencies, in much the same way as described previously for FDD. Suppose, as is generally the case, that the candidate frequency, $\omega_{p}$, corresponds to a peak in the 
first singular value. In each setup $q$, the first singular vector corresponding to the candidate frequency, $\left\{u_{1}\left(\omega_{p}, q\right)\right\}$, is then compared to the singular vectors (not only the first) at neighboring frequencies using the MAC. Since a particular vibration mode should still dominate the response at frequencies near to its natural frequency, the mode shape should be fairly well estimated by a singular vector over a range of frequencies on either side of the resonance peak. A single-degree-of-freedom (SDOF) bell is created by considering all the frequencies around a resonance peak for which a singular vector correlates well with the first singular vector at the peak. Note that the definition of what comprises a good correlation is arbitrary, and it is common to define a MAC rejection criterion, $\Omega$, which leads to an SDOF bell that captures as much of the resonance peak as possible. A typical starting value is 0.8 , though this depends on the quality of the data and the level of excitation of the particular mode being identified. The corresponding frequencies are denoted $\omega_{b}$. The SDOF bell is comprised of the singular values $s_{j}\left(\omega_{b}, q\right)$ corresponding to the singular vectors $\left\{u_{j}\left(\omega_{b}, q\right)\right\}$ that satisfy

$$
\operatorname{MAC}\left(\left\{u_{1}\left(\omega_{p}, q\right)\right\},\left\{u_{j}\left(\omega_{b}, q\right)\right\}\right) \geq \Omega
$$

In the case of well-separated modes, the singular vectors at neighboring frequencies that correlate well with the first singular vector at the resonant frequency, $\left\{u_{1}\left(\omega_{p}, q\right)\right\}$, are generally the first singular vectors, $\left\{u_{1}\left(\omega_{b}, q\right)\right\}$. However, when closely-spaced modes are present, higher singular vectors $\left(\left\{u_{2}\left(\omega_{b}, q\right)\right\},\left\{u_{3}\left(\omega_{b}, q\right)\right\}\right.$, etc.) need to be considered. In ARTeMIS, a sweep across all singular vectors is performed at each frequency to see if any of these correlate well with the reference mode shape. The resulting SDOF bell is only defined at frequencies near the selected peak; it is padded with zeros for the remaining frequencies, for which no singular vectors have a MAC value greater than the specified criterion (Brincker et al., 2001a). 
Figure 3.9 illustrates the identification of the SDOF bell for the first of three closely-spaced modes (for one particular measurement setup). The portion highlighted in red represents the SDOF bell. Note that to the left of the peak, the SDOF bell is comprised solely of the first singular value, indicating that the same mode dominates response for all frequencies to the left of the peak, while to the right of the peak, the SDOF bell moves to the second singular value as the adjacent vibration mode begins to dominate the response. Note that the MAC rejection criterion is selected so as to capture many data points on either side of the modal peak, yielding a smooth bell shape.

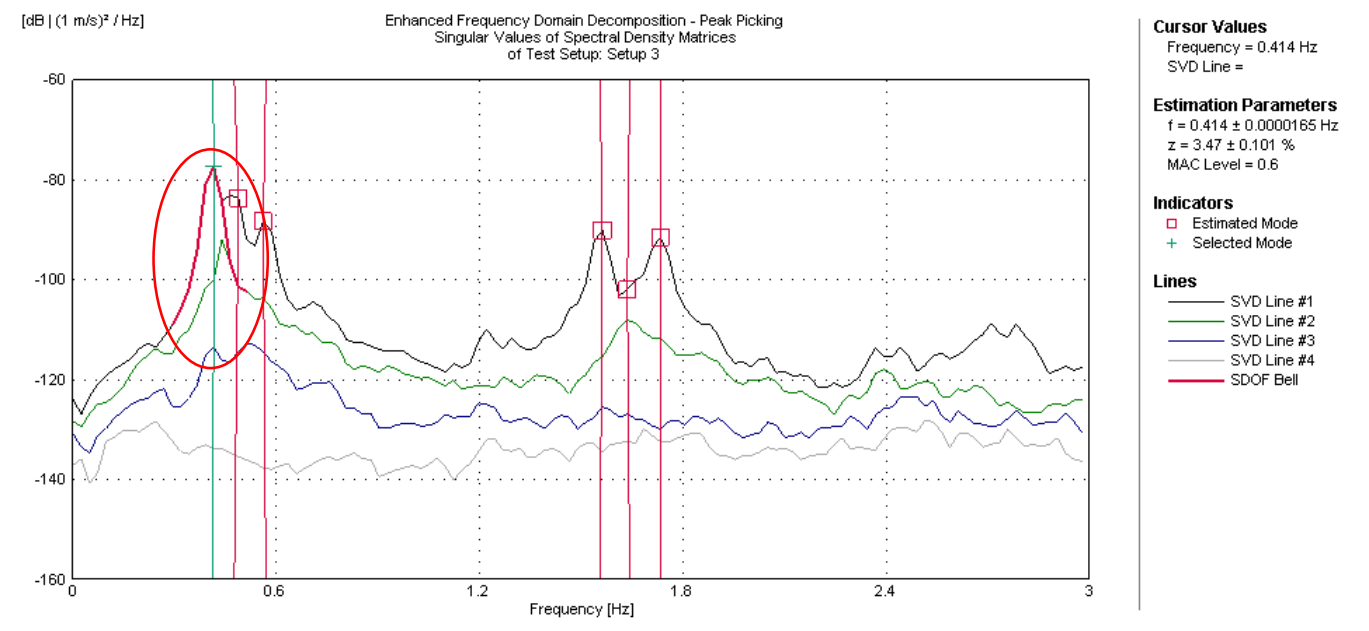

Figure 3.9: Example SDOF bell

Let us now examine how the SDOF bell is used to provide improved estimates of frequency and mode shape, as well as an estimate of modal damping. It should be noted that a different SDOF bell must be identified for each mode and for each setup. Though this involves significantly more effort than FDD, it also provides an opportunity to quantify the uncertainty in the various estimates by comparing the values obtained from different measurement setups. 
Improved estimate of frequency

For each mode, and for each setup, the identified SDOF bell is brought back to the time domain (or time lag domain) using the inverse fast Fourier transform (IFFT). From the Wiener-Khintchine relation (Bendat and Piersol, 2000), it follows that the time domain function thus obtained is an approximation to the SDOF autocorrelation function of the corresponding mode.

It can be shown that the autocorrelation function for the velocity of a linear SDOF oscillator subjected to white noise excitation is given by (Clough and Penzien, 2003)

$$
R_{v v}(\tau)=\frac{\pi \omega_{n} S_{0}}{2 k^{2} \xi}\left(\omega_{n}^{2} \cos \omega_{d} \tau-\frac{\omega_{n}^{2} \xi}{\sqrt{1-\xi^{2}}} \sin \omega_{d} \tau\right) \exp \left(-\xi \omega_{n} \tau\right)
$$

where $S_{0}$ represents the constant power spectral density of the white noise excitation, $k$ is the stiffness of the oscillator, $\omega_{n}$ is its undamped natural frequency, $\omega_{d}$ is its damped natural frequency, $\xi$ is its viscous damping ratio, and $\tau$ represents time lag. Hence, under the assumption of white noise excitation, the autocorrelation function should oscillate at a frequency equal to the damped natural frequency of the corresponding vibration mode, and should decay exponentially.

Figure 3.10 shows the SDOF autocorrelation function corresponding to the SDOF bell identified in Figure 3.9. 


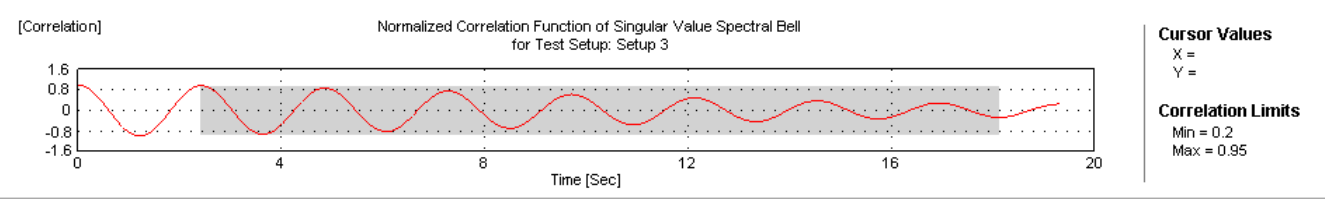

Figure 3.10: Example SDOF autocorrelation function

An improved estimate of the frequency can then be obtained by counting the zero crossings of the SDOF autocorrelation function (Brincker et al., 2001a). The zero crossings are plotted against time, as in Figure 3.11, and a linear regression is performed. The slope obtained from the regression represents the number of zero crossings per second, or twice the number of cycles per second since the function crosses zero twice per cycle. The frequency is therefore equal to half the slope.

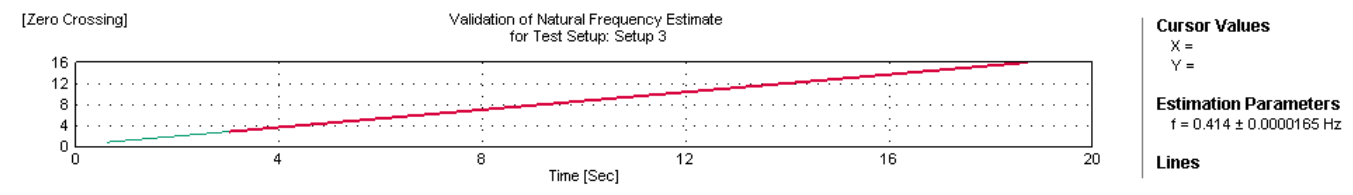

Figure 3.11: Improved estimate of frequency using zero crossings

Improved estimate of mode shape

An improved estimate of the mode shape is obtained by weighting the singular vectors from all frequencies included in the SDOF bell by their corresponding singular values,

$$
\left\{\phi\left(\omega_{p}\right)\right\}=\frac{\sum s_{i}\left(\omega_{b}\right)\left\{u_{i}\left(\omega_{b}\right)\right\}}{\sum s_{i}\left(\omega_{b}\right)}
$$

thus giving more weight to the singular values near the peak, while still performing an averaging operation over all relevant singular vectors. For conciseness, the index $q$ corresponding to each setup is dropped in the 
notation, but bear in mind that a different SDOF bell is identified for each mode and for each measurement setup. Hence, the improved mode shape vector calculated using equation 3.14 only involves the degrees of freedom included in that setup. The global mode shapes must be assembled from the mode shapes from each setup, as explained in section 3.2.2.2.

\section{Estimate of modal damping ratio}

The SDOF autocorrelation function decays exponentially in a manner similar to the free response of a linear SDOF oscillator with viscous damping. For this reason, the logarithmic decrement technique (Clough and Penzien, 2003), which is commonly applied to estimate the viscous damping ratio of an SDOF oscillator from its free vibration response, can be extended to the SDOF autocorrelation function. The estimation of the modal damping ratio from the SDOF autocorrelation function is explained in detail in Appendix A. In brief, the peaks in the autocorrelation function are identified along with the time at which they occur. The natural logarithms of these peak values are then plotted against time, as in Figure 3.12 , and a linear regression is performed.

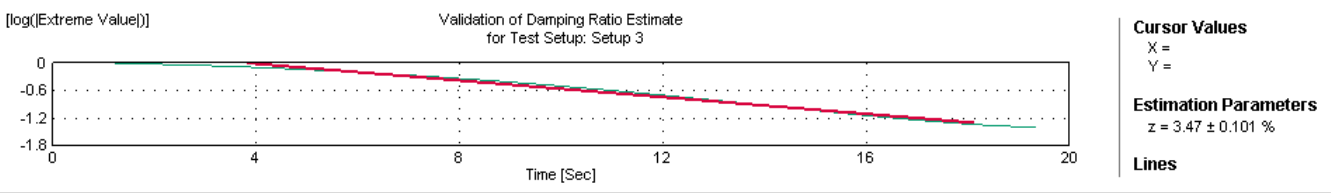

Figure 3.12: Estimate of viscous damping using logarithmic decrement

The slope $m$ obtained from the linear regression can be shown to be equal to the opposite of the ratio of the logarithmic decrement $\delta$ and the damped natural period $T_{d}$ (see Appendix A). Thus, the logarithmic decrement is calculated using 


$$
\delta=-m T_{d}
$$

The modal damping ratio $\xi$, defined as a fraction of the critical viscous damping value, is then calculated from the logarithmic decrement using (Brincker et al., 2001a)

$$
\xi=\frac{\delta}{\sqrt{\delta^{2}+4 \pi^{2}}}
$$

Estimate of uncertainty in frequency and damping estimates

As mentioned previously, in EFDD, a different SDOF bell must be identified for each mode and for each setup. Therefore, an estimate of frequency and damping of a particular mode of vibration is available for each setup. The results obtained from the different setups are compiled to provide a data set, from which a mean and standard deviation are calculated. The standard deviations of the frequency and modal viscous damping ratio evidently provide a measure of the uncertainty associated with each of these estimates.

The procedures described above were used to identify up to six vibration modes of 39 buildings in Montréal, including 27 RCSW buildings. The results are available in a separate report (Gilles, 2010). However, to illustrate the use of the enhanced frequency domain decomposition method, Appendix B shows the identification of the first two vibration modes from ambient tests in one of these buildings (RCSW-1). The next section shows that the results obtained using these methods are reliable and consistent. 


\section{Validation of experimental methods}

To ensure that the dynamic properties of the studied buildings, obtained using the methods described in the previous section, were reliable, these methods were validated using data from a structure with known properties. A numerical model was created using SAP2000 ${ }^{\circledR}$ (Computers and Structures, 2010). The "theoretical" linear modal parameters were computed by eigenvalue analysis. Then, artificial white noise loads were applied to the model and the velocity time histories of the various degrees of freedom were extracted, simulating measured ambient responses. These time histories were then analyzed to obtain the "experimental" dynamic properties of the structure. The experimental and theoretical modal properties were then compared to validate the algorithms.

Of course, the "experimental" data represented ideal conditions, where the input was white noise and the modes were orthogonal. In actual buildings, the damping is not likely to be proportional; therefore the modes are likely not perfectly orthogonal. Moreover, during an ambient vibration test, it is unlikely that the unknown and unmeasured forces exciting the structure will have equal energy content at all frequencies. Hence, data from ambient measurements are not expected to be as clear as the data obtained from this type of simulation. However, it will be shown that repeatable estimates were obtained nonetheless.

\subsection{Modal parameters of model structure}

\subsubsection{Description of numerical model}

To validate the modal identification procedure described previously, the simple numerical model of Figure 4.1 was created using SAP2000. It 
represents a two-storey plane frame in the $X Z$ plane. Both its in-plane and out-of-plane motions were considered, such that translation (in $X$ and $Y$ ) and torsion (about $Z$ ) modes could be studied, leading to a total of six dynamic degrees of freedom (DOF). Note that it is not necessary that the model be representative of an actual building; only that the "experimental" modal parameters compare well to those obtained from eigenvalue analysis in the model. For this reason, simple values were used for all the relevant parameters in the model, as described below. However, it was important that the modal frequencies and velocities be comparable to those from actual building tests. The velocities were typically in the range of $10^{-4}$ to $10^{-7} \mathrm{~m} / \mathrm{s}$, which is similar to the velocities typically measured in ambient tests (see section 3.1.1). Similarly, as will be shown in the next section, the modal frequencies were in the range of 1.5 to $6 \mathrm{~Hz}$, which agrees well with the range of frequencies identified for the buildings in this study.

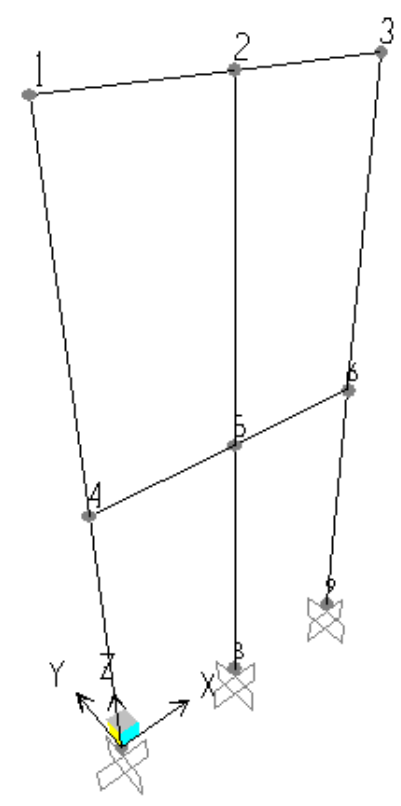

Figure 4.1: 6-DOF numerical model used to validate experimental methods

The bay width was $0.5 \mathrm{~m}$ and the storey height was $1 \mathrm{~m}$. All members were assigned $200 \times 300 \mathrm{~mm}$ rectangular sections, with the strong axis in 
the plane, made of an arbitrary material having a Young's modulus of 1 $\mathrm{MPa}$ and Poisson's ratio of 0 . Joints 1 through 6 were assigned a mass of $10 \mathrm{~kg}$ in the $X$ and $Y$ directions. To simulate ideal shear building behavior, the joints at each level were constrained to move horizontally as a rigid body using diaphragms at each floor level, assuming that the floors (beams and slabs) were infinitely rigid in their plane, and the joints at each level were restrained from rotating about each horizontal axis ( $X$ and $Y$ ), assuming that the floors could not tilt. Finally, fixed support conditions were used at the base.

\subsubsection{Theoretical linear modal parameters}

An eigenvalue analysis was performed to identify the theoretical modal frequencies of the model structure. Six vibration modes - two translation modes in each direction and two torsion modes - were obtained. The theoretical modal frequencies and damping ratios are shown in Table 4.1. In this table, $X 1$ and $X 2$ represent the first and second translation modes in the $X$ direction, $Y 1$ and $Y 2$ represent the first and second translation modes in the $Y$ direction, and $T 1$ and $T 2$ represent the first and second torsion modes. For conciseness, the mode shapes are not shown here, but the theoretical and experimental mode shapes were compared using the modal assurance criterion (see section 3.2.2.3). The model structure was assigned constant viscous damping of two percent critical in all vibration modes.

Table 4.1: Modal parameters of 6-DOF numerical model

\begin{tabular}{|c|c|c|}
\hline Mode & Freq [Hz] & Damp [\%] \\
\hline $\mathrm{Y} 1$ & 1.524 & 2.00 \\
\hline $\mathrm{T} 1$ & 1.920 & 2.00 \\
\hline $\mathrm{X} 1$ & 2.286 & 2.00 \\
\hline $\mathrm{Y} 2$ & 3.990 & 2.00 \\
\hline $\mathrm{T} 2$ & 5.026 & 2.00 \\
\hline $\mathrm{X} 2$ & 5.984 & 2.00 \\
\hline
\end{tabular}




\subsubsection{Experimental modal parameters}

The structure was then excited at joint 1 by independent white noise loads in each horizontal direction ( $X$ and $Y$ ), for a duration of five minutes. These white noise loading functions were created using the normally-distributed pseudorandom number generator, randn, in MATLAB. Technically, the loads are only "quasi-white" since a true white noise signal has equal energy content across the entire spectrum, which requires an infinitely long signal.

Since measurements in actual buildings required a roving sensor approach, a similar procedure was adopted to simulate the fact that not all degrees of freedom of the model structure could be measured simultaneously. Five load cases were defined, each containing different white noise loads in both the $X$ and $Y$ directions applied at joint 1. Joint 1 was selected as the reference location, and for each load case, the velocity time histories (in the $X$ and $Y$ directions) of the reference joint and the roving joint were sampled at $1000 \mathrm{~Hz}$. Each joint was selected in turn as the roving joint. Table 4.2 shows, for each load case, the different white noise loads used and the degrees of freedom for which the responses were extracted. The letter ( $\mathrm{X}$ or $\mathrm{Y}$ ) represents the direction, and the number (1 to 6 ) represents the joint. These time histories were then used in the FDD and EFDD procedures in ARTeMIS to obtain the experimental modal parameters.

Table 4.2: 6-DOF numerical model - Load cases and response DOF

\begin{tabular}{|c|c|c|c|c|}
\hline Load case & Load-X & Load-Y & Reference DOF & Roving DOF \\
\hline case 1 & wn1 & wn2 & $\mathrm{X} 1, \mathrm{Y} 1$ & $\mathrm{X} 2, \mathrm{Y} 2$ \\
\hline case 2 & wn3 & wn4 & $\mathrm{X} 1, \mathrm{Y} 1$ & $\mathrm{X} 3, \mathrm{Y} 3$ \\
\hline case 3 & wn5 & wn6 & $\mathrm{X} 1, \mathrm{Y} 1$ & $\mathrm{X} 4, \mathrm{Y} 4$ \\
\hline case 4 & wn7 & wn8 & $\mathrm{X} 1, \mathrm{Y} 1$ & $\mathrm{X} 5, \mathrm{Y} 5$ \\
\hline case 5 & wn9 & wn10 & $\mathrm{X} 1, \mathrm{Y} 1$ & $\mathrm{X} 6, \mathrm{Y} 6$ \\
\hline
\end{tabular}


Since the highest frequency of any of the vibration modes in the model structure was $5.984 \mathrm{~Hz}$, the frequency range was limited to $0-10 \mathrm{~Hz}$ by decimating the signals by a factor of 50 , leading to an effective sampling rate of $20 \mathrm{~Hz}$. A frequency spacing of $0.01953 \mathrm{~Hz}$ was selected by specifying 512 frequency lines in this frequency range. Figure 4.2 shows the average normalized singular value plot of these data. Though four singular values were calculated at each frequency, since each measurement setup (load case) involved four response DOF, Figure 4.2 only shows the first two. Six resonance peaks in the first singular value (upper black line) are clearly visible, corresponding to the six vibration modes of the model structure.

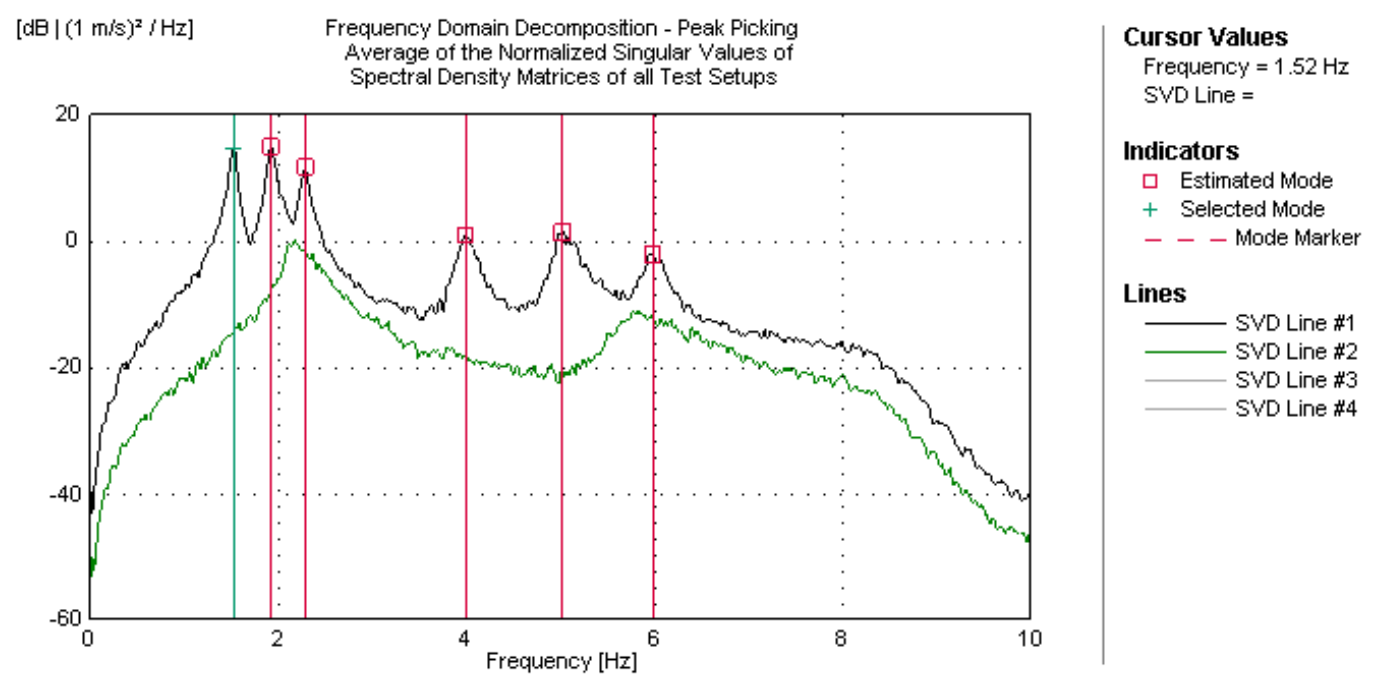

Figure 4.2: Singular value plot using results from 6-DOF numerical model

Both the FDD and EFDD methods were used to estimate the experimental modal parameters. In the FDD procedure, the six peaks were selected as potential modal frequencies, and the corresponding mode shapes were estimated from the first singular vector at those frequencies. 
In the EFDD procedure, a different MAC rejection value was selected for each setup (load case), and for each mode, to obtain a good representation of the modal peak, as described in section 3.2.2.3. For example, in Figure 4.3, corresponding to the singular value plot of the first load case, a MAC rejection value of 0.528 was deemed to adequately capture the SDOF bell function for the first mode. The SDOF bell function thus obtained was then transformed back to the time domain to generate the SDOF autocorrelation function for that mode. Different correlation limits were set for each setup, and for each mode, to capture the decaying portion of the autocorrelation function. Figure 4.4 shows the autocorrelation function for the first mode, and the first load case. Here, maximum and minimum correlation values of 0.95 and 0.2 were selected. This portion of the autocorrelation function was then used to calculate improved estimates of the frequency (Figure 4.5) and to estimate the modal damping ratio (Figure 4.6).

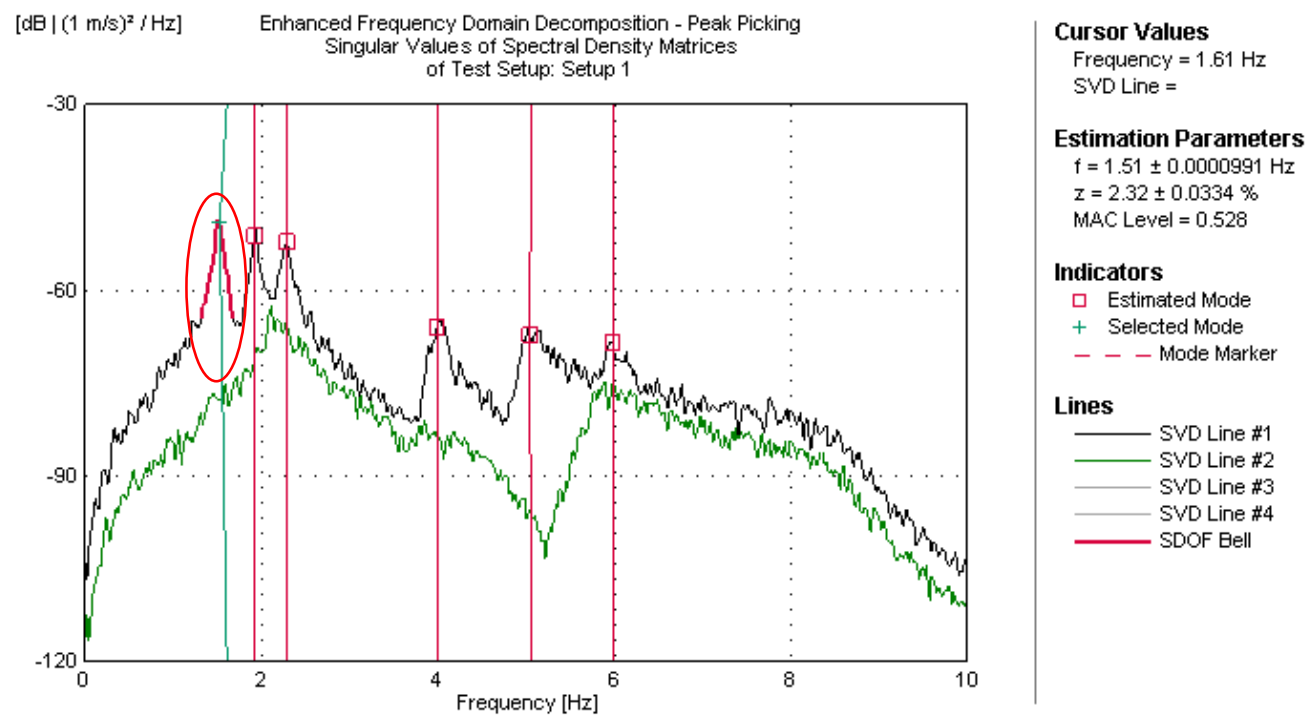

Figure 4.3: SDOF bell for first mode of model structure (load case 1) 


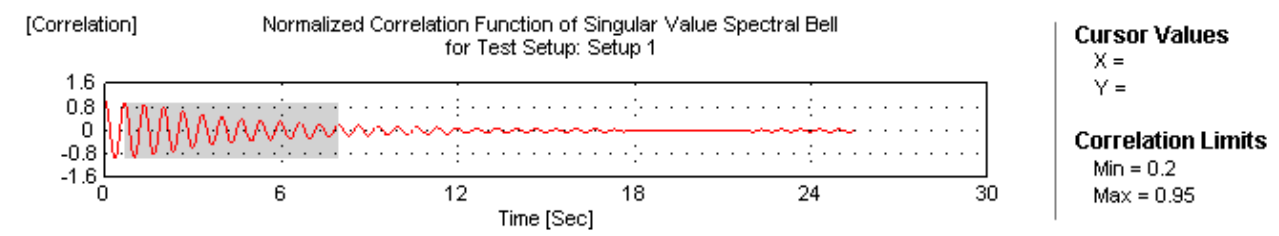

Figure 4.4: SDOF autocorrelation function for first mode of model structure (load case 1)

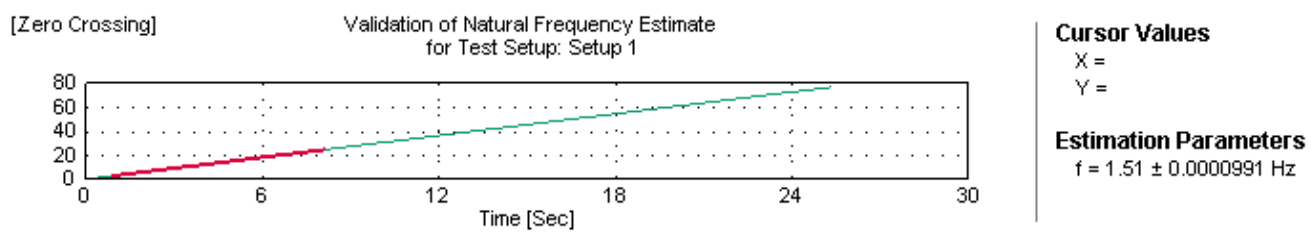

Figure 4.5: Improved estimate of frequency of first mode of model structure (load case 1)

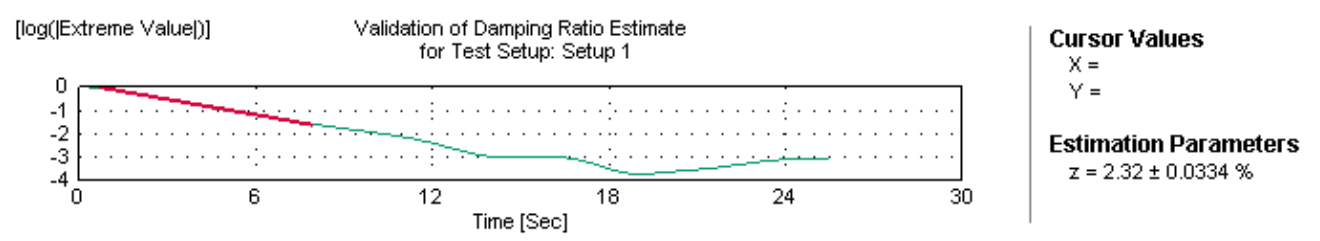

Figure 4.6: Damping estimate for first mode of model structure (load case 1)

Since this was done for each mode and for each load case, different values of frequency and damping were obtained for each load case (recall that there were five load cases in all). As a result, the mean value and standard deviation of each modal parameter were computed by averaging the values obtained for all load cases. Table 4.3 shows the mean values and standard deviations of the frequencies and damping ratios of the six vibration modes, estimated using EFDD. The standard deviations of the frequencies are small, indicating that the modal frequencies estimated for each setup were consistent. On the other hand, the standard deviations of the modal damping ratios were larger, indicating that the damping ratios estimated from different load cases were more variable. The frequencies obtained from FDD were very similar. 
Table 4.3: Modal parameters of model structure obtained from EFDD

\begin{tabular}{|c|c|c|c|c|}
\hline Mode & Freq [Hz] & Std. Freq [Hz] & Damp [\%] & Std. Damp [\%] \\
\hline Y1 & 1.522 & 0.009 & 2.290 & 0.270 \\
\hline T1 & 1.919 & 0.004 & 2.294 & 0.294 \\
\hline X1 & 2.276 & 0.010 & 2.035 & 0.471 \\
\hline Y2 & 3.994 & 0.028 & 2.417 & 0.667 \\
\hline T2 & 5.053 & 0.015 & 2.522 & 0.410 \\
\hline X2 & 5.981 & 0.020 & 1.907 & 0.383 \\
\hline
\end{tabular}

\subsubsection{Comparison of theoretical and experimental modal parameters}

To assess the accuracy of the modal identification procedure, the experimental frequency and damping values were directly compared to the values from the numerical model. The comparison was done based on the results from EFDD. Table 4.4 shows that the theoretical and experimental frequencies compared very well, with the largest error being 0.55 percent for the second torsion mode. The damping ratios were not as well estimated, with relative errors as large as 26 percent, as shown in Table 4.5. This agrees with past findings, which indicate that damping estimates are typically prone to large uncertainty. This may be the result of imperfect assumptions about the input forces (that they are stationary, with a flat spectrum across the frequency range of interest), leakage, or insufficient averages in the estimation of spectral density (Brownjohn, 2003), or in this case partial identification of the SDOF bells in certain setups (Rainieri et al., 2010).

Table 4.4: Comparison of theoretical and experimental modal frequencies

\begin{tabular}{|c|c|c|c|}
\hline \multirow{2}{*}{ Mode } & \multicolumn{2}{|c|}{ Frequency [Hz] } & \multirow{2}{*}{} \\
\cline { 2 - 3 } & Theoretical & Experimental $^{*}$ & \% Error \\
\hline Y1 & 1.524 & 1.522 & 0.12 \\
\hline T1 & 1.920 & 1.919 & 0.03 \\
\hline X1 & 2.286 & 2.276 & 0.43 \\
\hline Y2 & 3.990 & 3.994 & 0.11 \\
\hline T2 & 5.026 & 5.053 & 0.55 \\
\hline X2 & 5.984 & 5.981 & 0.05 \\
\hline
\end{tabular}

* Note: Experimental frequency values identified using EFDD 
Table 4.5: Comparison of theoretical and experimental modal damping ratios

\begin{tabular}{|c|c|c|c|}
\hline \multirow{2}{*}{ Mode } & \multicolumn{2}{|c|}{ Damping [\%] } & \multirow{2}{*}{} \\
\cline { 2 - 3 } & Theoretical & Experimental & \% Error \\
\hline Y1 & 2.00 & 2.29 & 14.50 \\
\hline T1 & 2.00 & 2.29 & 14.70 \\
\hline X1 & 2.00 & 2.04 & 1.75 \\
\hline Y2 & 2.00 & 2.42 & 20.85 \\
\hline T2 & 2.00 & 2.52 & 26.10 \\
\hline X2 & 2.00 & 1.91 & 4.65 \\
\hline
\end{tabular}

The accuracy of the experimental mode shape estimates was investigated using the modal assurance criterion (MAC) between the model and EFDD mode shapes. Table 4.6 shows that the mode shapes were generally estimated accurately; that is, the MAC values are close to one along the main diagonal. Note however that the fourth and fifth modes (Y2 and T2) were not estimated accurately. This is likely because these modes were only weakly excited in certain load cases; consequently, the SDOF bell could not be properly identified in these load cases. However, Table 4.7 shows that the mode shapes were estimated more accurately using FDD.

Table 4.6: Comparison of theoretical and experimental mode shapes (EFDD)

\begin{tabular}{|c|c|c|c|c|c|c|c|}
\hline & \multicolumn{6}{|c|}{ EFDD-Modes } \\
\hline & & $1.522 \mathrm{~Hz}$ & $1.919 \mathrm{~Hz}$ & $2.276 \mathrm{~Hz}$ & $3.994 \mathrm{~Hz}$ & $5.053 \mathrm{~Hz}$ & $5.981 \mathrm{~Hz}$ \\
\hline \multirow{6}{*}{ 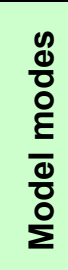 } & $1.524 \mathrm{~Hz}$ & 0.985 & 0.006 & 0.000 & 0.003 & 0.001 & 0.001 \\
\hline & $1.920 \mathrm{~Hz}$ & 0.017 & 0.958 & 0.014 & 0.001 & 0.001 & 0.000 \\
\hline & $2.286 \mathrm{~Hz}$ & 0.000 & 0.000 & 1.000 & 0.000 & 0.000 & 0.000 \\
\hline & $3.990 \mathrm{~Hz}$ & 0.000 & 0.009 & 0.050 & 0.663 & 0.040 & 0.037 \\
\hline & $5.026 \mathrm{~Hz}$ & 0.021 & 0.012 & 0.001 & 0.078 & 0.805 & 0.008 \\
\hline & $5.984 \mathrm{~Hz}$ & 0.000 & 0.000 & 0.006 & 0.000 & 0.000 & 0.993 \\
\hline
\end{tabular}

Table 4.7: Comparison of theoretical and experimental mode shapes (FDD)

\begin{tabular}{|c|c|c|c|c|c|c|c|}
\hline & \multicolumn{6}{|c|}{ FDD-Modes } \\
\hline & & $1.523 \mathrm{~Hz}$ & $1.914 \mathrm{~Hz}$ & $2.285 \mathrm{~Hz}$ & $3.984 \mathrm{~Hz}$ & $5.020 \mathrm{~Hz}$ & $5.977 \mathrm{~Hz}$ \\
\hline \multirow{6}{*}{ 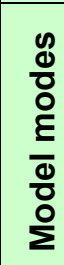 } & $1.524 \mathrm{~Hz}$ & 0.992 & 0.007 & 0.000 & 0.000 & 0.000 & 0.000 \\
\hline & $1.920 \mathrm{~Hz}$ & 0.008 & 0.991 & 0.000 & 0.000 & 0.000 & 0.000 \\
\hline & $2.286 \mathrm{~Hz}$ & 0.000 & 0.000 & 1.000 & 0.000 & 0.000 & 0.000 \\
\hline & $3.990 \mathrm{~Hz}$ & 0.006 & 0.010 & 0.000 & 0.976 & 0.007 & 0.000 \\
\hline & $5.026 \mathrm{~Hz}$ & 0.003 & 0.006 & 0.000 & 0.008 & 0.982 & 0.000 \\
\hline & $5.984 \mathrm{~Hz}$ & 0.000 & 0.001 & 0.005 & 0.001 & 0.000 & 0.992 \\
\hline
\end{tabular}


Based on visual inspection of the mode shapes, which can be animated in $A R T e M I S$, the fourth and fifth modes were more coherent when estimated using FDD. For this reason, for all building tests, the modal properties were extracted using both FDD and EFDD and the more coherent mode shapes were retained.

From the above results, it is clear that both FDD and EFDD can be relied upon to provide reliable estimates of the natural frequencies and mode shapes of a structure. Estimates of damping are not quite as accurate, but still provide a measure of how much energy is dissipated in each vibration mode.

\subsection{Modal parameters of real buildings}

As mentioned previously, data from ambient studies in actual buildings are not as ideal as the simulated data from a numerical model. Since it is impossible to know the theoretical modal parameters of an actual building, there is no way of ensuring that the modal parameters estimated from an ambient vibration study are accurate. The only way to check that reliable estimates can be obtained is by ensuring that the results are repeatable.

In 16 of the 39 buildings studied, and whose dynamic properties were reported in Gilles (2010), ambient vibration tests were performed on two or three different days. These buildings are Hyb-2, Hyb-3, RCMRF-4, RCMRF-6, RCMRF-7, RCSW-3, RCSW-4, RCSW-7, RCSW-8, RCSW-9, RCSW-10, RCSW-11, RCSW-16, RCSW-18, RCSW-20, and SMRF-2. For each of these buildings, the results from different days were compared to ensure that reliable and consistent results could be expected from ambient tests. For each building, as many as six vibration modes were identified, corresponding to the first two translation modes in each direction, and the first two torsion modes. A total of 81 vibration modes were considered. 
Note that SMRF-2 is a steel moment-resisting frame building, which was considered here to ensure that the dynamic characteristics identified on different days were consistent and reliable, but was not otherwise considered in this study. In contrast, the buildings identified by the prefixes Hyb- and RCSW- rely at least partially on concrete shear walls for their lateral resistance, and the results for these buildings are included in section 5 .

Figure 4.7 shows the frequencies of the 81 vibration modes identified on each day plotted against the average frequency (obtained by averaging the frequencies obtained on different days). The frequencies of all modes, identified on any given day, diverge very little from the average value, indicating that frequency estimates obtained from ambient tests on different days are nearly identical. In fact, the standard deviation of frequency estimates identified on different days for the same vibration mode of the same building varied between 0.000 and $0.085 \mathrm{~Hz}$. Further, the best-fit from a linear regression analysis was a straight line with unit slope and intercept of zero, and had a coefficient of determination, $R^{2}$, of 1.000 .

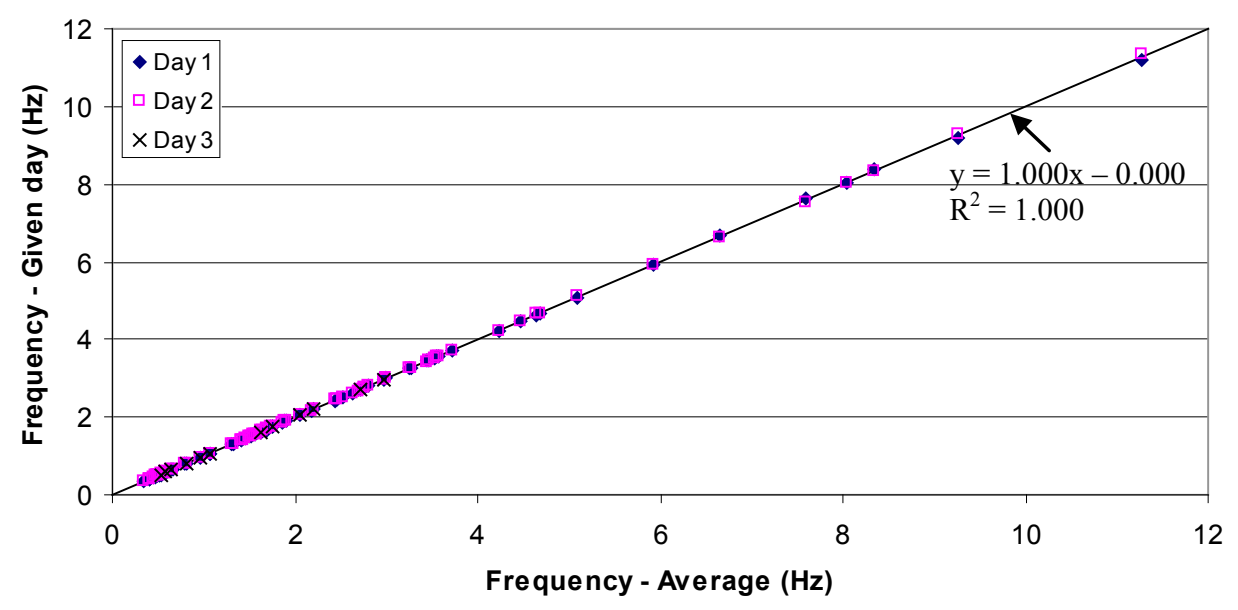

Figure 4.7: Comparison of modal frequencies identified on different days 
Similarly, Figure 4.8 shows the damping ratios identified on different days, for each of the 16 buildings, plotted against the average damping ratio. The standard deviation of damping estimates identified on different days for the same vibration mode of the same building varied between 0.001 and 0.993 percent of critical viscous damping. Note that the values on any given day diverge significantly more from the average value, as compared to the frequencies, which confirms past findings that damping estimates are more uncertain. However, the values obtained on different days are still quite consistent, as evidenced by the good fit of the regression line.

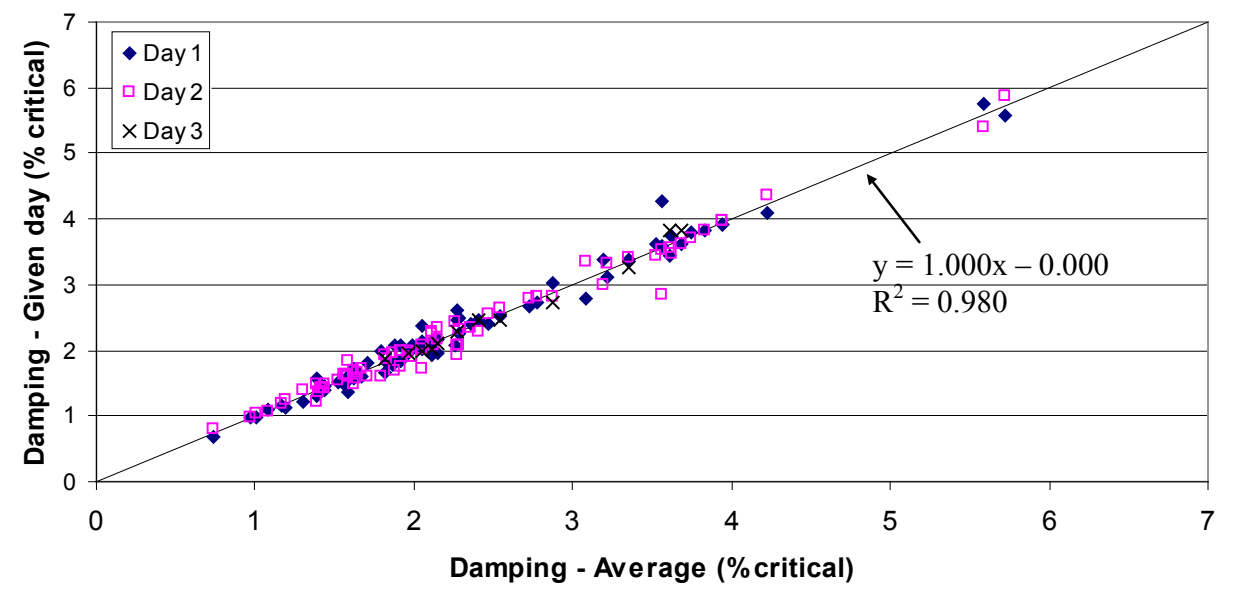

Figure 4.8: Comparison of modal damping ratios identified on different days

Finally, Figure 4.9 shows the modal assurance criteria (MAC) between mode shape estimates on different days for ten vibration modes from buildings Hyb-2, Hyb-3, and RCMRF-4. Most MAC values are greater than 0.9 , indicating a strong correlation between mode shape estimates obtained on different days. Unfortunately, for all other buildings, measurements on different days involved different degrees of freedom, or the relative phases of the various DOF could not be trusted due to synchronization problems. Nevertheless, from these ten vibration modes, the mode shape estimates on different days are shown to be consistent. 


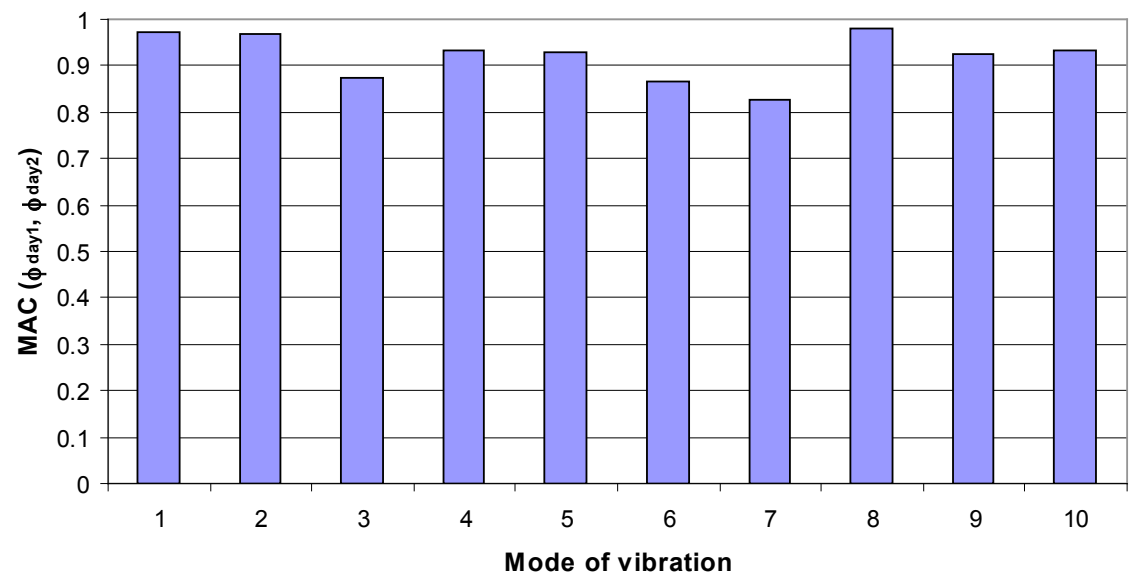

Figure 4.9: Comparison of mode shapes identified on different days

In summary, enhanced frequency domain decomposition (EFDD) and frequency domain decomposition (FDD) were found to provide relatively accurate estimates of the frequencies, damping ratios, and mode shapes of a six-DOF model structure, obtained from its simulated responses. And, though there is no way of ensuring that these estimates are accurate, the modal parameters of 16 buildings, identified on different days, were found to be consistent. Therefore, the modal parameters identified using the methods described in section 3 are reliable and consistent. 


\section{Results and discussion}

\subsection{Fundamental period of RCSW buildings}

As we have seen, the fundamental period of a building plays a crucial role in its response to dynamic loads, such as strong winds or earthquake ground motions. Therefore, to predict the internal member forces, lateral deflections, and accelerations of a building subjected to design-level dynamic loads, reliable methods to estimate its fundamental period are required.

To this end, many empirical equations have been proposed to estimate the fundamental period of RCSW buildings, ranging in complexity from simple equations based on building height to more complex equations which incorporate the dimensions of individual structural elements, as discussed in section 2.3.1. The equation adopted in the 2005 National Building Code of Canada (NBCC 2005) was developed based on the measured periods of buildings in California. Since local design and construction practices, as well as seismicity, may affect the lateral stiffness of a building, it is unclear whether these equations adequately estimate the fundamental periods of RCSW buildings in Canada.

Let us now evaluate the accuracy of the equation adopted in the NBCC 2005 to estimate the fundamental period of RCSW buildings using the fundamental periods of 27 RCSW buildings, which were identified from ambient vibration tests. These data are also used to assess whether alternative equations can improve the prediction of the fundamental period for these buildings. Since modern building codes explicitly require the estimation of the fundamental period to calculate design seismic loads, the focus here is mainly on the estimation of fundamental period for seismic 
analysis and design. However, much of the following discussion can be extended to the dynamic effects of wind as well.

\subsubsection{Measured fundamental period data for RCSW buildings}

For seismic analysis and design of buildings in Canada, empirical fundamental period equations would ideally be based on a large data set comprised of buildings in Canada, having a broad range of building heights, whose periods were measured during earthquake ground motions. These data would be most representative of the fundamental periods that could be expected during design-level seismic ground motions, as these periods have been shown to vary with the excitation magnitude (see section 2.3.1.7). However, to the author's knowledge, no such data exist for Canadian buildings; in fact, aside from the data presented below, even around the world, few such data are available in open literature.

In light of this, it is debatable whether it is preferable to develop period equations using only local period data, which reduces the quantity of data, or incorporating also period data from buildings in other regions, which do not necessarily reflect design and construction practices in Canada. Further, some may argue that only periods measured during moderate to strong shaking events should be considered. But again, this would limit the data to a few buildings in other regions. It is the author's opinion that, until better data are available, periods from ambient vibration studies represent the best information available for RCSW buildings in Canada and should be considered in evaluating the accuracy of period equations for these types of buildings. For this reason, two data sets are considered to evaluate commonly used period equations for buildings in which shear walls provide the main resistance to lateral loads: 
Data set DS-1: Period data from this study (Table D-1, Appendix D), corresponding to low-amplitude motions of reinforced concrete shear wall buildings in Montréal

Data set DS-2: Larger data set comprised of period data from Goel and Chopra (1998), Kijewski-Correa and Pirnia (2007), Lee et al (2000), Farsi and Bard (2004), Ghrib and Mamedov (2004), and this study (Table D-2, Appendix D)

Table D-1 (Appendix D) shows the measured periods of 27 multi-storey, reinforced concrete buildings in Montréal, whose lateral stiffness is provided primarily by shear walls. Ambient vibration measurements were performed in these buildings between June 2007 and August 2009, and their dynamic properties were extracted using the methods described in section 3. More details about the buildings and the identification of their dynamic properties are available in a separate report (Gilles, 2010). Note that the measured data represent the dynamic properties of each building system, which includes the building itself (structural and non-structural elements and content), as well as its foundation and the surrounding soil.

In usual practice, individual shear walls are connected together at each floor level by concrete slabs, or through coupling beams, which may transfer significant shear forces between adjacent walls. In fact, coupled walls are very common in the building core of multi-storey buildings, where openings for elevator and stairwell entrances are required. Also, structural designers sometimes choose to use a combination of shear walls and moment-resisting frames to resist lateral loads, as the latter system allows larger open spaces.

From the buildings studied, four distinct types of lateral load-resisting systems (LLRS), all involving shear walls, were identified: uncoupled 
shear walls (USW), coupled shear walls (CSW), combination of coupled and uncoupled shear walls (CUSW), and hybrid wall-frame (Hyb). Walls were considered to be uncoupled when connected only through floor slabs, whereas they were considered to be coupled when connected by beams that were significantly deeper than the floor slabs and that lay in the same plane as the walls. Hybrid wall-frame systems involve a combination of concrete shear walls (coupled or uncoupled) and concrete moment-resisting frames.

In most cases, the lateral load-resisting system was identified from structural plans. However, detailed structural plans could not be obtained for all buildings. For buildings RCSW-3, RCSW-4, and RCSW-15, the presence of concrete shear walls was instead confirmed from other sources (Gilles, 2010); but the exact layout and dimensions of the walls could not be obtained. Similarly, for buildings RCSW-21, RCSW-22, and RCSW-23, no structural plans were available, and the presence of concrete shear walls could not be confirmed from other sources; however, inspection of public areas suggested the presence of concrete walls in the central core. These six buildings were categorized simply as RCSW.

\subsubsection{Effect of different types of shear walls}

By virtue of their size, when shear walls are present, they are likely to contribute the most to the lateral stiffness of a building. However, when walls are coupled, or when moment frames are designed to resist a portion of the lateral loads, the overall lateral stiffness, and thus the natural periods of vibration, of the building may be affected. In the interest of evaluating different period equations, let us first examine whether it is justified to treat the data from the four types of lateral load-resisting systems as a single data set. 
Figure 5.1 shows a plot of the measured periods against building height for buildings with different types of walls.

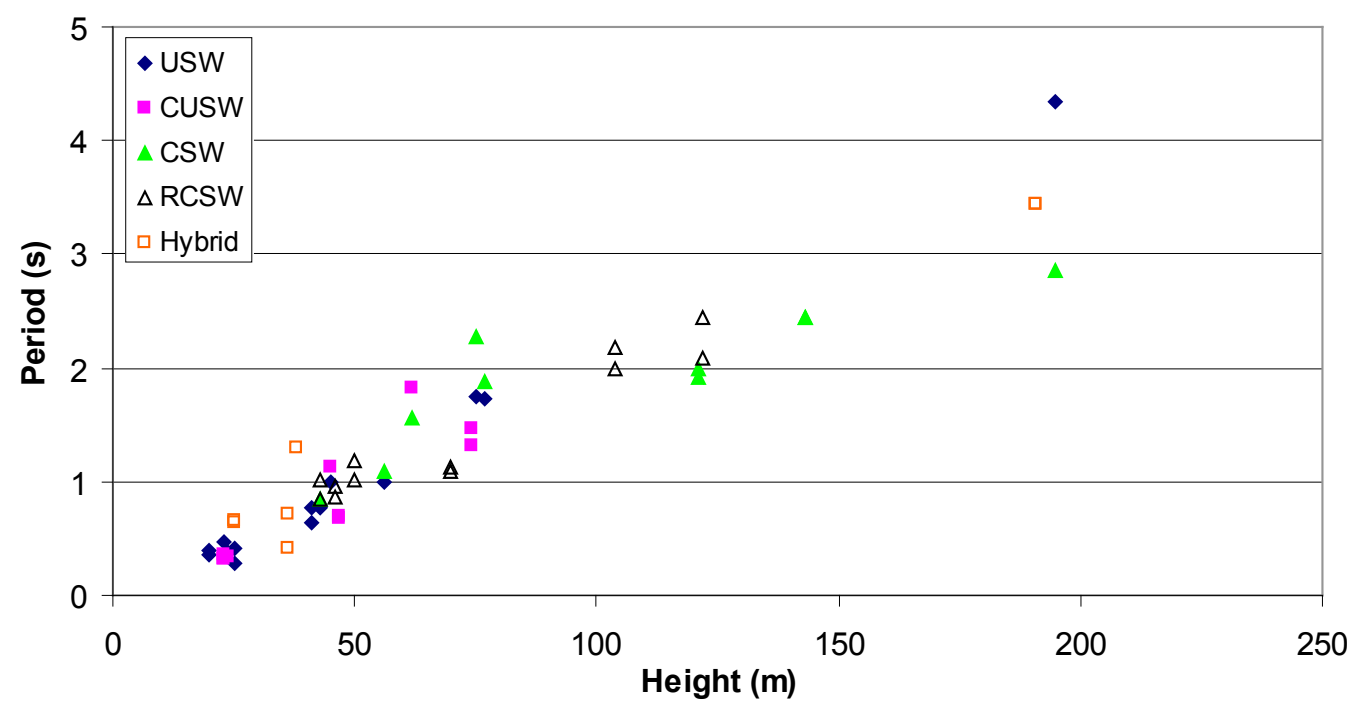

Figure 5.1: Fundamental periods of buildings in Montréal with different types of shear walls

In the range from 20 to $100 \mathrm{~m}$, there are several data points corresponding to each type of lateral load-resisting system, and there does not appear to be any major difference between the periods of buildings with different types of walls. It thus seems reasonable to pool the period data from all these buildings into a single data set. However, to confirm this, a more rigorous statistical analysis was carried out to determine whether the data for USW and the remaining buildings were likely to represent samples of different populations. By comparing the regression line corresponding to USW buildings only to that obtained considering the data from all remaining buildings, at the 95 percent significance level, this analysis confirmed that the measured periods of USW buildings were not significantly different than those of the remaining buildings. Hence, the fundamental periods of all these lateral load-resisting systems are considered as a single data set to evaluate different period equations. The statistical analysis is described in more detail in Appendix E. 


\subsubsection{Comparison with data from other studies}

Several other studies have been performed to identify the fundamental periods of concrete shear wall buildings in other countries, such as the United States (Goel and Chopra, 1998), Korea (Kijewski-Correa and Pirnia, 2007; Lee et al., 2000), France (Farsi and Bard, 2004), and Azerbaijan (Ghrib and Mamedov, 2004). The data from these studies are presented in Table D-2 (Appendix D). Figure 5.2 shows a comparison of building periods from the various studies, including this one. With the exception of the data from Kijewski-Correa and Pirnia, the building periods from the other studies were limited to building heights less than $70 \mathrm{~m}$. It is clear that the data from this study are particularly useful because they extend that range up to nearly $200 \mathrm{~m}$.

Note that the Goel and Chopra data represent periods measured during earthquake ground motions, whereas the remainder of the periods were obtained from ambient vibration records. Since periods from strong, or even moderate, shaking are expected to be longer than those from lowamplitude ambient motions, it is not surprising that the periods from Goel and Chopra appear to be slightly longer than those from the other studies, except those from Lee et al. However, local design and construction practices, as well as seismic hazard, also affect lateral stiffness (Hong and Hwang, 2000). This may explain why the buildings in Korea, studied by Lee et al during low-amplitude ambient vibrations, appear to have longer periods than their counterparts in other areas. 


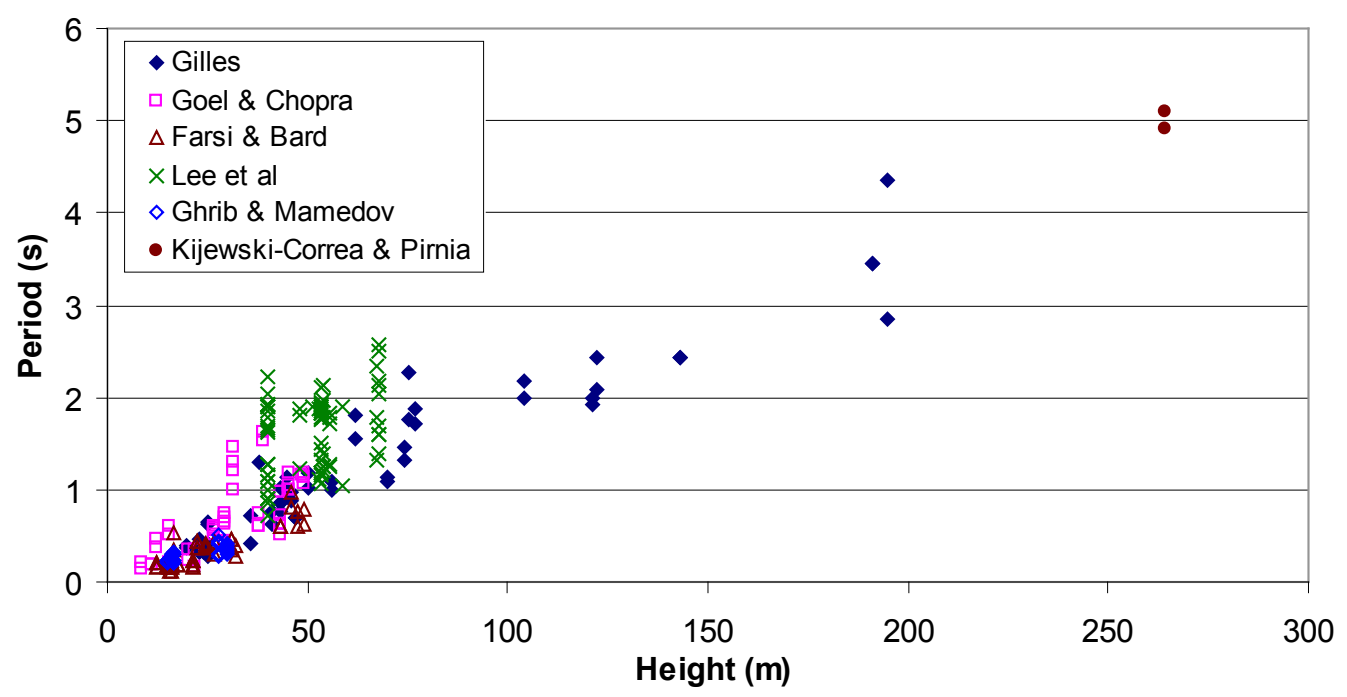

Figure 5.2: Fundamental periods of SW buildings from various studies (DS-2)

\subsubsection{Evaluation of code formula}

Recall from section 2.3.1.1 that the 2005 edition of the National Building Code of Canada (NBCC 2005) provides the following equation (rewritten and renumbered here for convenience) to estimate the fundamental period of buildings in which lateral forces are resisted by reinforced concrete shear walls:

$$
T_{1}=0.05 h^{3 / 4}
$$

where $h$ is the building height in metres. The same equation is suggested in several international codes (ASCE, 2005; BSSC, 2003; ICC, 2006). Recall also that the form of this equation, with the exponent of $3 / 4$ applied to the building height, was derived from a theoretical analysis under a number of simplifying assumptions. It can be shown however that the form of equation 5.1, particularly the value of the exponent, would be different if different, but equally plausible, assumptions were made in the analysis (Goel and Chopra, 1997). 
Let us now evaluate equation 5.1 using the measured periods of real buildings to determine whether the continued use of the $3 / 4$ exponent is justified, or whether another equation can improve the fit to the measured data.

Figure 5.3 shows the periods of 27 reinforced concrete shear wall buildings in Montréal (data set DS-1), plotted against building height. Also shown are the code period equation (solid red line), the maximum allowable period equation (dotted green line), and the best-fit line obtained from constrained regression analysis (dashed blue line); this analysis is explained in more detail in the next section. Similarly, Figure 5.4 shows the global period data (data set DS-2) plotted against building height.

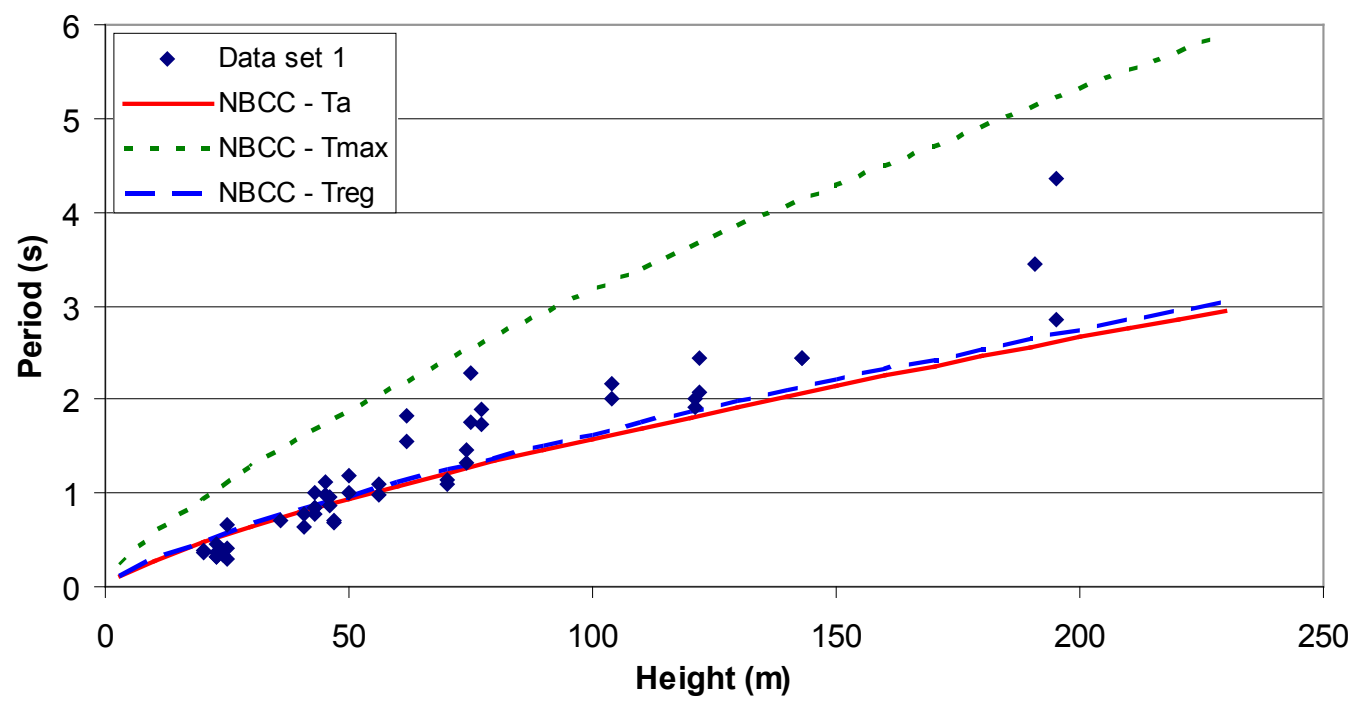

Figure 5.3: Evaluation of code period formula using measured fundamental periods of RCSW buildings in Montréal (DS-1) 


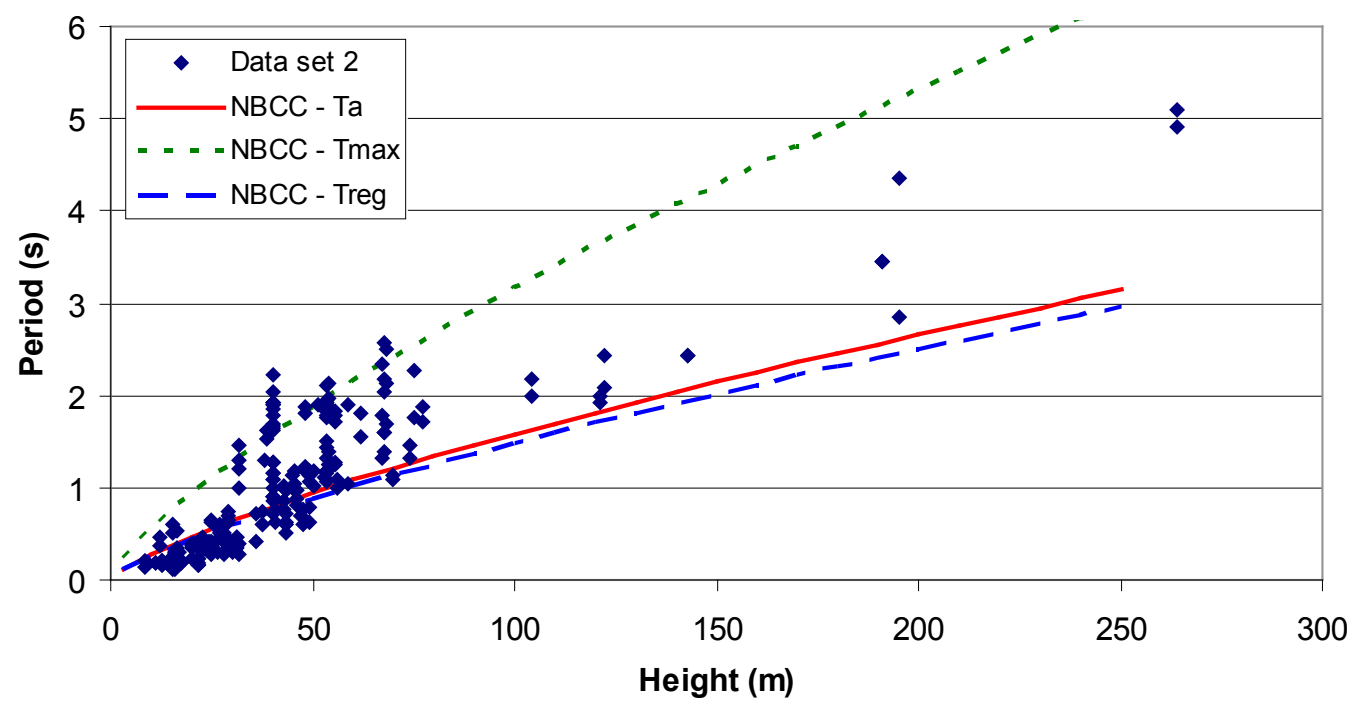

Figure 5.4: Evaluation of code period formula using measured fundamental periods of SW buildings (DS-2)

Since the design acceleration spectra used to calculate seismic loads generally decrease with increasing fundamental period (see section 2.2.1), the code period equation was developed with the aim of underestimating the fundamental period in most cases. Thus, it is not meant to represent the mean value - though it nearly does in this case - and it cannot be expected to fit the data very well. Therefore, evaluating the accuracy of the code equation relative to other candidate equations by comparing measured and predicted values would not be appropriate. A more suitable approach to compare the goodness-of-fit of different equations is to consider the mean value curve, which can easily be obtained by regression analysis.

\subsubsection{Regression analysis}

To compare the accuracy of different period equations, based solely on building height, regression analyses were performed considering the two data sets presented above. The candidate equations were all of the form 


$$
T_{1}=a h^{b}
$$

This equation was recast into linear form by taking the natural logarithm of each side:

$$
y=\alpha+b x
$$

where $y=\ln \left(T_{1}\right), x=\ln (h)$, and $\alpha=\ln (a)$.

The variance of $y$ was assumed constant for all values of $x$. Figures 5.5 and 5.6 show the periods from data sets DS-1 and DS-2 in logarithmic format, in addition to the mean value (best-fit), mean minus one standard deviation, and mean plus one standard deviation curves. From these figures, the assumption of constant variance seems reasonable since the spread of the data in $y$ does not appear to be dependent upon the value of $x$. Hence, the transformed $x$ and $y$ data are amenable to standard linear regression techniques, as described for example in Ang and Tang (1975).

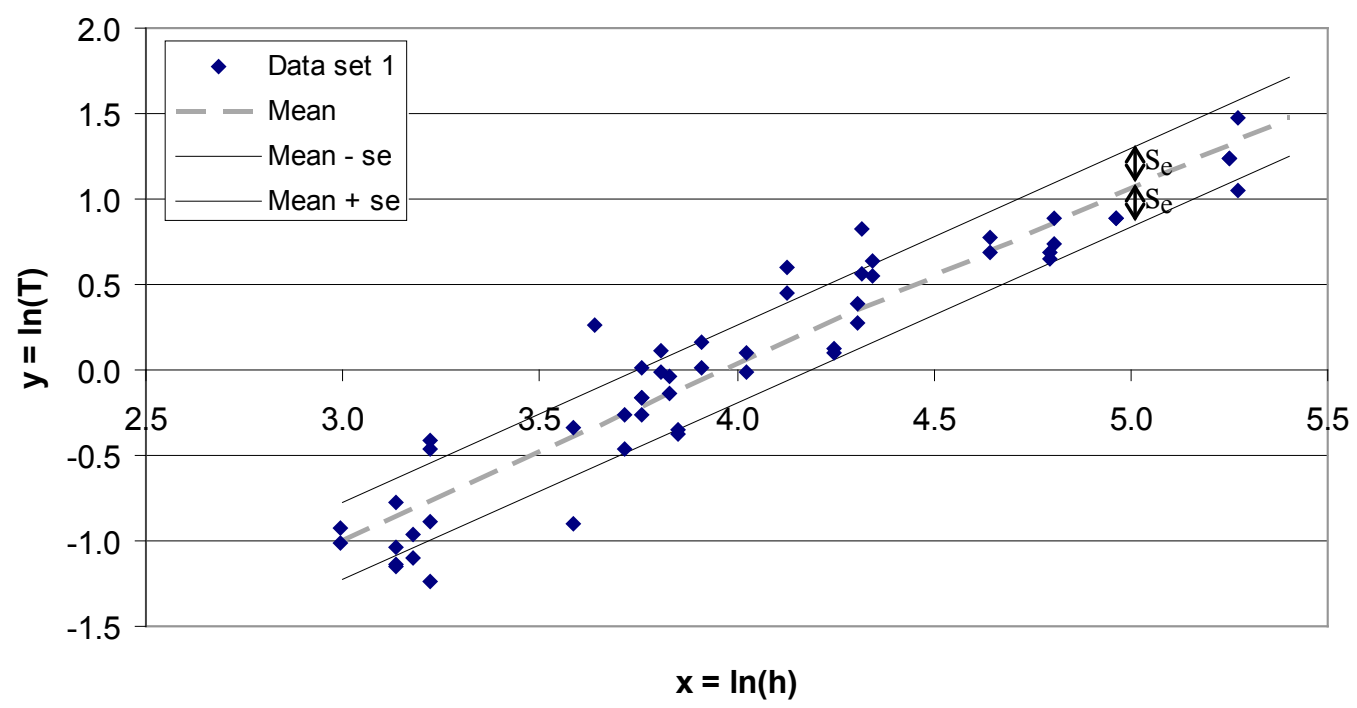

Figure 5.5: Logarithmic plot of measured fundamental periods of RCSW buildings in Montréal (DS-1) 


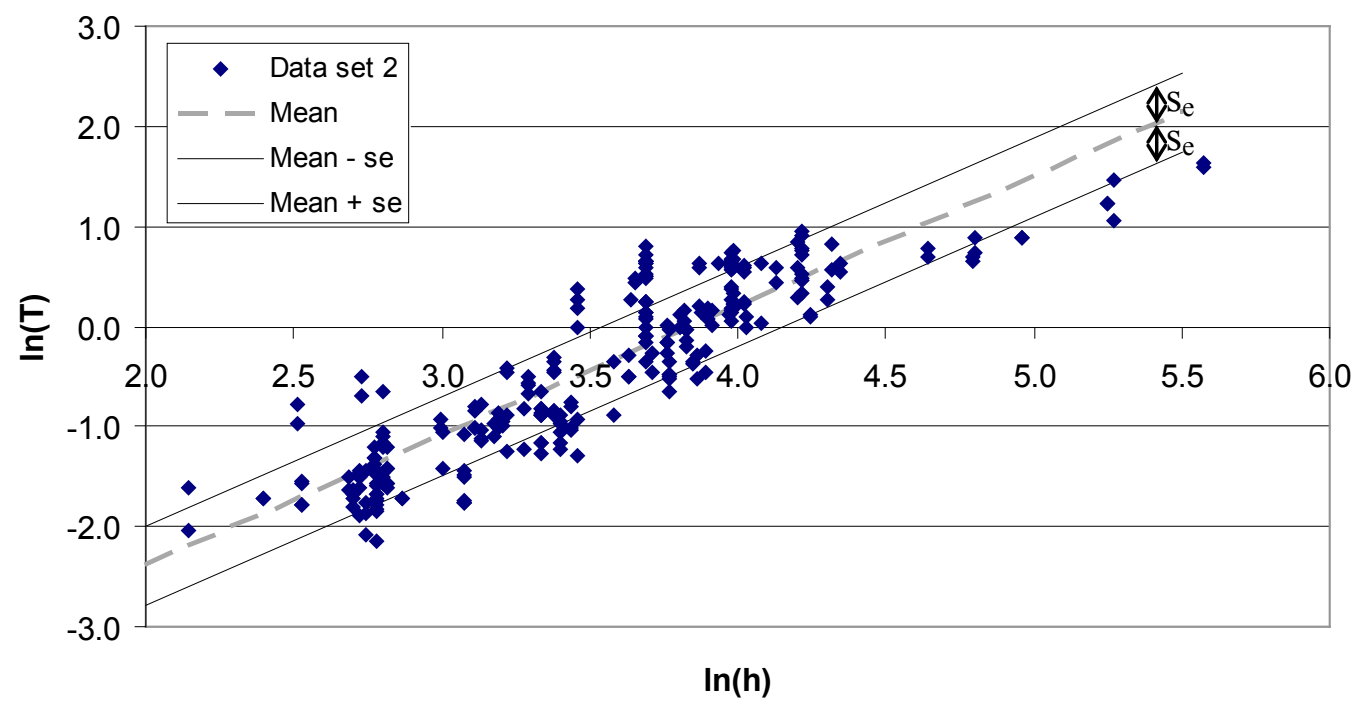

Figure 5.6: Logarithmic plot of measured fundamental periods of SW buildings (DS-2)

Though different values of the exponent $b$ were considered for each analysis, the regression parameter $\alpha$ leading to the best fit to the measured data was always calculated using

$$
\alpha=\bar{y}-b \bar{x}
$$

where $\bar{x}$ and $\bar{y}$ represent the mean value of $x$ and $y$. The coefficient $a$ of the best-fit curve was then obtained from

$$
a=\exp (\alpha)
$$

The quality of the fit was evaluated using two indicators: the coefficient of determination $\left(R^{2}\right)$ and the standard error of estimate $\left(s_{e}\right)$. The coefficient of determination varies between zero - indicating no correlation - and one - indicating perfect correlation. Since all the candidate equations were linear in the transformed variables $x$ and $y$, but not in the original variables 
$h$ and $T_{l}$, the coefficient of determination was calculated using $x$ and $y$, as follows:

$$
R^{2}=1-\frac{S S_{e}}{S S_{t}}
$$

where

$$
\begin{aligned}
& S S_{e}=\sum_{i=1}^{n}\left[y_{i}-\left(\alpha+b x_{i}\right)\right]^{2} \\
& S S_{r}=\sum_{i=1}^{n}\left[\left(\alpha+b x_{i}\right)-\bar{y}\right]^{2} \\
& S S_{t}=S S_{e}+S S_{r}
\end{aligned}
$$

in which $i$ indicates the $i^{\text {th }}$ data point, and $n$ indicates the total number of data points. The standard error of estimate provides an estimate of the spread of the data relative to the regression line, and approaches the standard deviation when the number of data points is large. It is calculated using

$$
s_{e}=\sqrt{\frac{S S_{e}}{n-2}}
$$

Recall that the variance - and hence the standard deviation - of $y$ was assumed to be constant for all values of $x$. Thus, equation 5.10 provides an estimate of the standard deviation of $y$ for any given value of $x$.

Tables 5.1 and 5.2 summarize the results of the regression analyses for both data sets, which are described in more detail below. 
Table 5.1: Results of regression analyses using period data for RCSW buildings in Montréal (DS-1)

\begin{tabular}{|c|c|c|c|c|}
\hline Type & Coefficient b & Best-fit & $\mathbf{s}_{\mathbf{e}}$ & $\mathbf{R}^{\mathbf{2}}$ \\
\hline Constrained & 0.750 & $T_{1}=0.052 h^{0.75}$ & 0.296 & 0.738 \\
\hline Unconstrained & 1.032 & $T_{1}=0.017 h^{1.032}$ & 0.229 & 0.898 \\
\hline Constrained & 1.000 & $T_{1}=0.019 h$ & 0.230 & 0.892 \\
\hline
\end{tabular}

Table 5.2: Results of regression analyses using global period data for SW buildings (DS-2)

\begin{tabular}{|c|c|c|c|c|}
\hline Type & Coefficient b & Best-fit & $\mathbf{S}_{\mathbf{e}}$ & $\mathbf{R}^{\mathbf{2}}$ \\
\hline Constrained & 0.750 & $T_{1}=0.047 h^{0.75}$ & 0.515 & 0.447 \\
\hline Unconstrained & 1.292 & $T_{1}=0.007 h^{1.292}$ & 0.391 & 0.806 \\
\hline Constrained & 1.000 & $T_{1}=0.020 h$ & 0.431 & 0.672 \\
\hline
\end{tabular}

First, the accuracy of the code period equation was assessed by constraining the exponent $b$ to $3 / 4$. Considering only buildings in Montréal (DS-1), the constrained regression yielded a coefficient $a$ of 0.052 , with a coefficient of determination of 0.738 and a standard error of estimate of 0.296. Considering instead global period data (DS-2), a similar analysis yielded a coefficient of 0.047 , with a coefficient of determination of 0.447 and a standard error of estimate of 0.515 . Clearly, the best-fit curves for both data sets are quite similar. However, the variability is significantly greater when the global period data are considered. This is not surprising since the global data encompass buildings in different regions, having different design and construction practices, some of whose periods were measured during earthquake ground shaking. Finally, note that the code period equation fits the measured data rather poorly, as indicated by relatively low coefficients of determination and relatively high standard errors of estimate for both data sets.

To determine whether any equation based solely on building height could improve the fit to the measured period data, an unconstrained regression was performed. Rather than being constrained to $3 / 4$, the exponent $b$ in equation 5.2 was allowed to assume the value corresponding to the bestfit in the least-squares sense, and was calculated as 


$$
b=\frac{\sum_{i=1}^{n} x_{i} y_{i}+n \bar{x} \bar{y}}{\sum_{i=1}^{n} x_{i}^{2}-n \bar{x}^{2}}
$$

Aside from this difference, the regression analysis was performed as described above. Table 5.1 shows that increasing the exponent $b$ to 1.032 , while lowering the constant term $a$ to 0.017 , would improve the fit to the data from Montréal buildings (DS-1). Such an equation yields a coefficient of determination of 0.898 (rather than 0.738 for the code equation), and a standard error of estimate of 0.229 (rather than 0.296 ), which clearly is a significant improvement over the code equation. In contrast, the fit to the global period data (DS-2) would be improved considerably by increasing the exponent $b$ to 1.292 and using a constant term $a$ of 0.007 , as shown in Table 5.2.

Figures 5.7 and 5.8 show how the different equations fit the measured data.

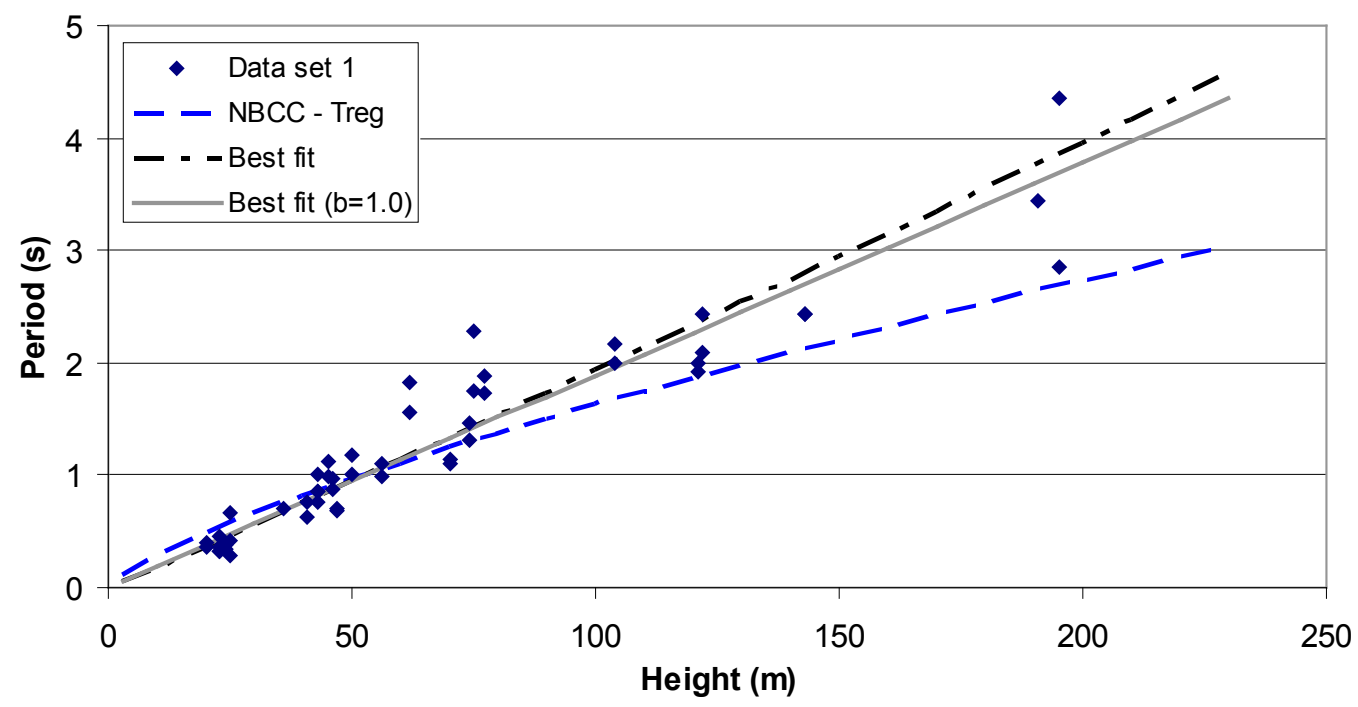

Figure 5.7: Results of regression analyses on fundamental period (DS-1) 


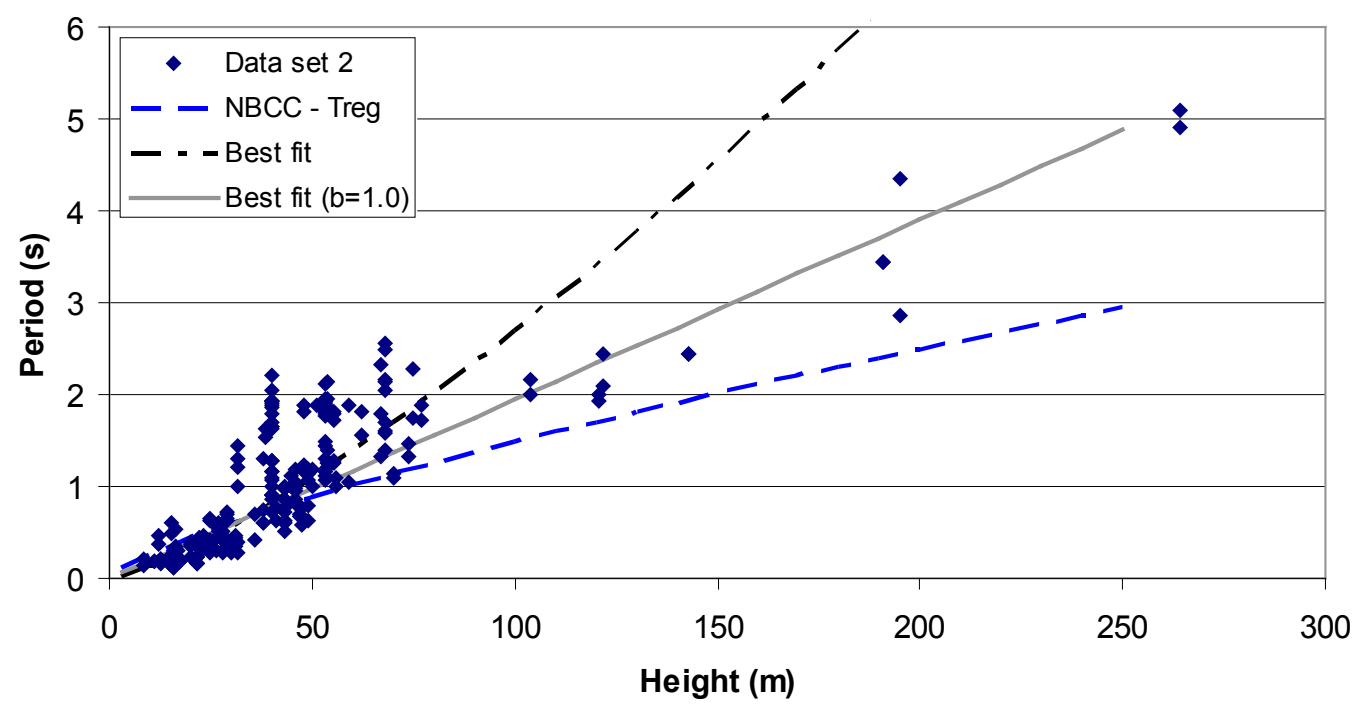

Figure 5.8: Results of regression analyses on fundamental period (DS-2)

In light of the fact that the form of equation 5.1 - with an exponent of $3 / 4$ applied to the building height - was derived based on a theoretical analysis using a number of simplifying assumptions, and that equally plausible assumptions would lead to a different exponent, constraining the exponent to $3 / 4$ does not appear to be justified. Since a better fit to the experimental data for both data sets can be achieved by increasing the exponent, if the period is to continue to be determined based on height alone, an equation similar to that obtained from the unconstrained regression analyses should be used. However, which equation should be used? The equation that best fits the measured period data for Montréal buildings (DS-1) or that which best fits the global period data (DS-2)?

To guide the decision, note that the best-fit curve for the global period data (DS-2) leads to unreasonably long periods for buildings having a height greater than 100 metres. In contrast, the best-fit curve for data set DS-1 should predict the periods reasonably well, even for buildings as tall as 200 metres. For this reason, the equation corresponding to the best fit to the data for Montréal buildings is used as reference. However, the 
precision of this equation suggests that the fundamental period can be predicted quite accurately, while Figures 5.7 and 5.8 clearly show that the prediction is not always that accurate.

The exponent was therefore rounded to unity, and a final constrained regression analysis was performed for each data set. This yields a coefficient $a$ of 0.019 for data set DS- 1 and 0.020 for DS-2. Evidently, the fits are slightly worse than for the unconstrained regression analyses, but still significantly better than for an equation of the same form as the current code equation. Notice also the excellent agreement between the curves obtained for both data sets.

Let us now examine whether alternative, more complex, equations improve the fit to the measured period data.

\subsubsection{Evaluation of alternative formulae}

It is evident that any equation based solely on building height cannot capture the increased stiffness contributed by additional walls or walls of larger dimensions, and therefore cannot be expected to fit measured data perfectly. Recognizing this, several researchers have proposed alternative equations making use of wall dimensions. Though these may offer the potential to better predict building periods, it should be noted that they require a priori knowledge of shear wall dimensions. Several such equations are studied here to see whether they can be reliably applied to buildings in Canada.

\subsubsection{Goel and Chopra formula}


Recall from section 2.3.1.3 that Goel and Chopra (1998) derived a model based on flexural and shear deformations in a uniform cantilever, and calibrated to the measured periods of nine RCSW buildings in California, to predict the fundamental periods of such buildings. The resulting equations have been adopted in several codes as an alternative method to compute the fundamental periods of concrete and masonry shear wall buildings. Let us rewrite and renumber these equations here for convenience:

$$
T_{1}=\frac{0.0062 h}{\sqrt{A_{e}}}
$$

where

$$
A_{e}=\frac{100}{A_{B}} \sum_{i=1}^{N W}\left(\frac{h}{h_{i}}\right)^{2} \frac{A_{i}}{\left[1+0.83\left(\frac{h_{i}}{D_{i}}\right)^{2}\right]}
$$

Recall that these equations were derived based on the following assumptions:

- All shear walls are uncoupled,

- The building plan is symmetric in the lateral direction under consideration and does not change along the building height, and

- The shear walls have the same dimensions along the building height.

In practice, these assumptions are rarely satisfied. As evident in Table D-1 (Appendix D), few buildings in the Montréal database have only uncoupled shear walls in either direction. This jeopardizes the general applicability of 
the above equation. Moreover, in many tall buildings, the plan dimensions decrease in the upper storeys. This is addressed in the codes where this equation has been adopted (ASCE, 2005; BSSC, 2003) by replacing the building plan area by the building plan area at the base of the building. This makes the equation more general, but may decrease its accuracy, since it is permitted to be applied to buildings that do not satisfy the basic assumptions used to develop the equations. Finally, regarding the last assumption, Goel and Chopra recognized that it is common practice to reduce the size of walls in the upper storeys of a building, since the shear force distribution decreases with height, and suggested that the wall dimensions at the base of the building could be used. It is clear that these simplifying assumptions affect the general applicability and accuracy of equation 5.12 .

\section{Inconsistency in Goel and Chopra formula}

Before evaluating whether equation 5.12 can be used to improve the prediction of the period for buildings in Canada, it is important to point out a significant inconsistency in equations 5.12 and 5.13 concerning buildings in which some shear walls do not extend to the top of the building. To illustrate this, consider two identical buildings having three shear walls of identical plan dimensions, arranged in a symmetrical fashion, contributing stiffness in a particular direction. For simplicity, suppose that Figure 5.9 represents the plan dimensions $(20 \mathrm{~m} \times 30 \mathrm{~m})$ and shear wall layout of both buildings. In the first building, all three walls extend to the top of the building, while in the second, two of these walls (W1 and W3) extend only up to half the building height. Suppose also that the height of both buildings is $30 \mathrm{~m}$, the length of the walls is equal to one fifth their height (6 $\mathrm{m}$ ), and the thickness of each wall is equal to $0.250 \mathrm{~m}$, which implies that each wall has an area equal to $1.5 \mathrm{~m}^{2}$, or $0.0025 \mathrm{~A}_{B}$. 


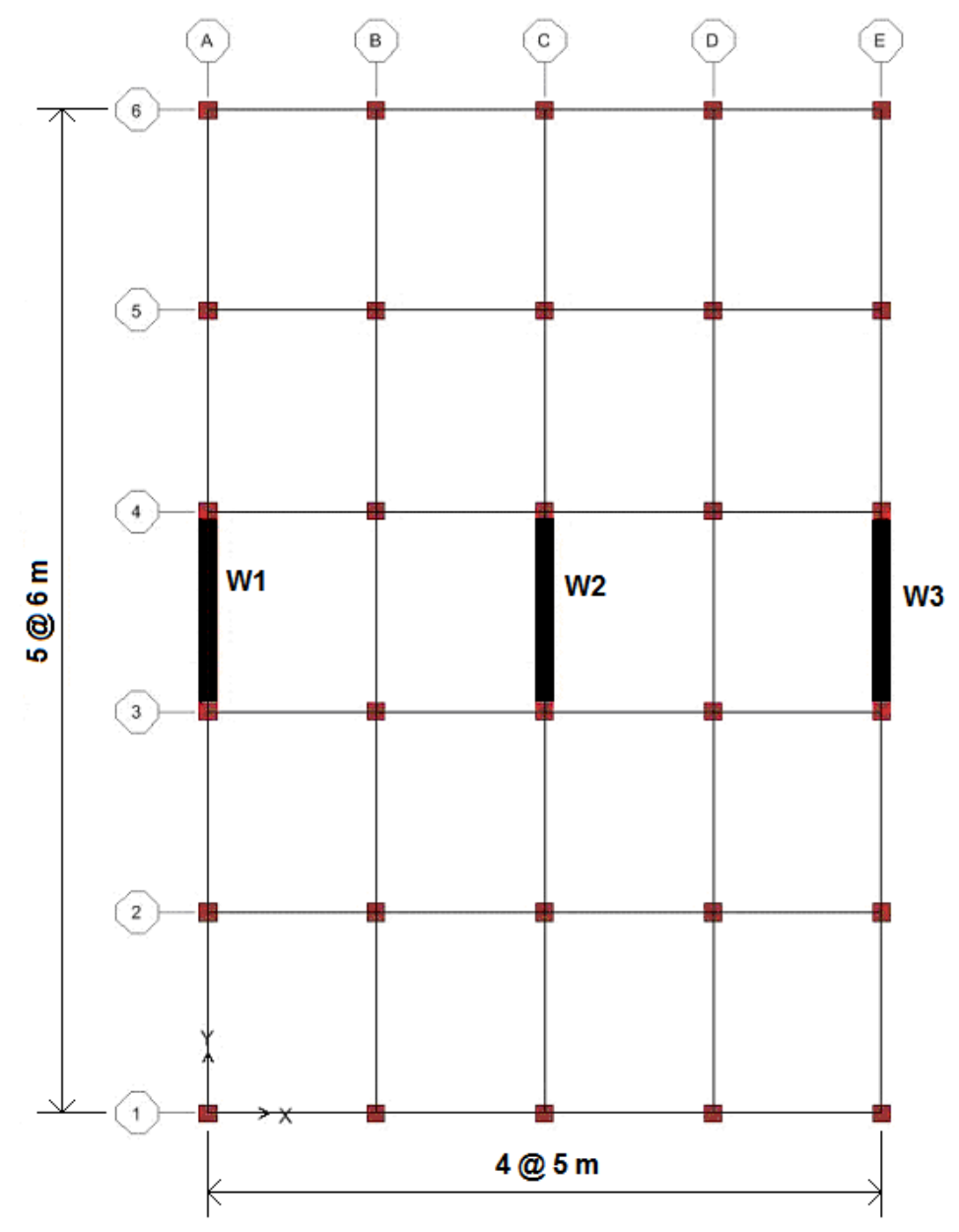

Figure 5.9: Plan view of model building to illustrate inconsistency in Goel and Chopra formula

\section{Building 1:}

$$
\begin{aligned}
& h=30 \mathrm{~m} \\
& A_{B}=600 \mathrm{~m}^{2} \\
& D_{1}=D_{2}=D_{3}=6 \mathrm{~m} \\
& t_{1}=t_{2}=t_{3}=0.250 \mathrm{~m} \\
& A_{1}=A_{2}=A_{3}=1.5 \mathrm{~m}^{2} \\
& h_{1}=h_{2}=h_{3}=h=30 \mathrm{~m}
\end{aligned}
$$

\section{Building 2:}

$$
\begin{aligned}
& h=30 \mathrm{~m} \\
& A_{B}=600 \mathrm{~m}^{2} \\
& D_{1}=D_{2}=D_{3}=6 \mathrm{~m} \\
& t_{1}=t_{2}=t_{3}=0.250 \mathrm{~m} \\
& A_{1}=A_{2}=A_{3}=1.5 \mathrm{~m}^{2} \\
& h_{1}=h_{3}=h / 2=15 \mathrm{~m} \\
& h_{2}=h=30 \mathrm{~m}
\end{aligned}
$$


For Building 1, in the longitudinal $(Y)$ direction, equation 5.13 would yield an equivalent shear area $A_{e}$ of $0.0345\left(\% A_{B}\right)$, leading to a fundamental period estimate of 1.00 seconds using equation 5.12. For the second building, $A_{e}$ would be $0.335\left(\% A_{B}\right)$, leading to a period of 0.32 seconds. The shorter period for Building 2 would suggest that it is considerably stiffer than Building 1, despite having less wall area in the upper five storeys. Of course, if different building or wall dimensions were used, the exact values would be different. However, the trend would be the same; that is, decreasing the wall area in the upper storeys would lead to shorter periods. Conceptually, this appears to be an erroneous result; but let us demonstrate this using the results of a numerical model.

Buildings 1 and 2 were modeled using SAP2000. Since we are interested in establishing which building should have a longer fundamental period, and not in accurately determining the fundamental period of each building, the material and section properties used in the models are of little importance. For this reason, uncracked concrete stiffness properties were assumed for all structural elements. Further, the lateral stiffness of the columns was neglected by assigning stiffness property modifiers to the column elements. The material and section properties, as well as other modeling details, are summarized below.

\section{Concrete material properties: $\quad$ Section properties:}

$$
\begin{array}{ll}
E=24.6 \mathrm{GPa} & \text { Beams: } 400 \text { (wide) } \times 600 \text { (deep) [mm] } \\
v=0.2 & \text { Columns: } 500 \times 500[\mathrm{~mm}] \\
\rho=2400 \mathrm{~kg} / \mathrm{m}^{3} & \text { Walls: } 6000 \text { (long) } \times 250 \text { (thick) [mm] } \\
& \text { Slabs: } 150 \mathrm{~mm} \text { (thick) }
\end{array}
$$

\section{Other modeling details:}

- All joints at the base were assumed to be fixed, 
- Floor slabs were modeled as rigid diaphragms by constraining all joints at a given floor level using a diaphragm constraint,

- Walls were modeled as shell elements, with a 10 (horizontal) x 5 (vertical) element meshing pattern

- No additional masses or loads were added to the bare frame, and

- The analysis was performed in the $Y$ direction only. Therefore, translation in the $X$ and $Z$ directions, as well as rotation about the $X$, $Y$ and $Z$ axes were not considered.

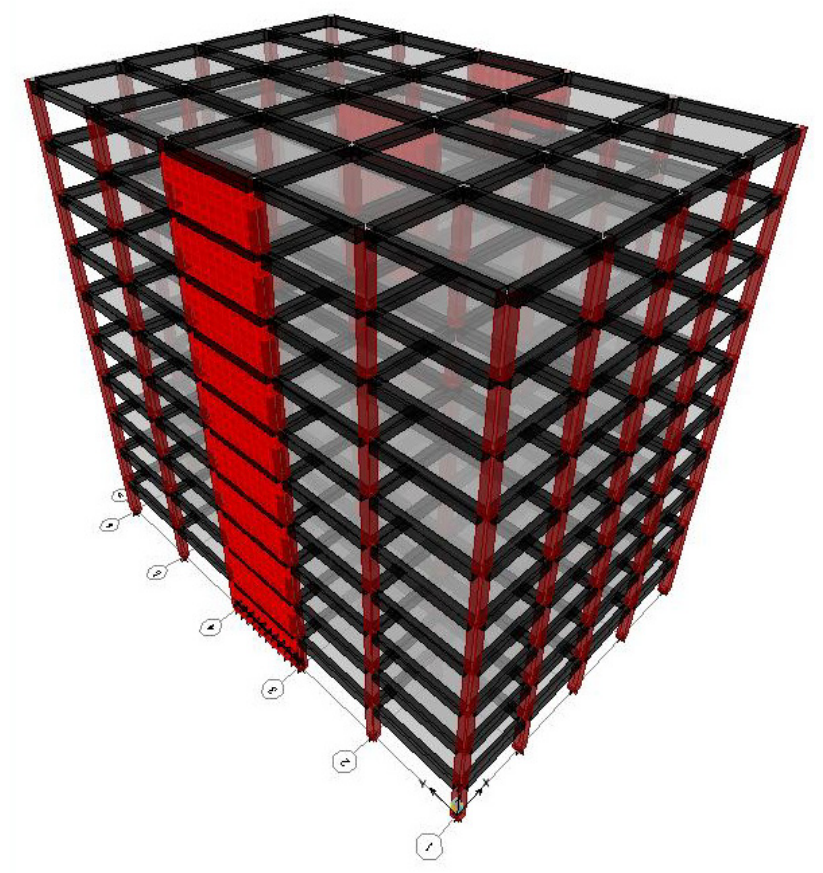

Figure 5.10: 3D view of Building 1 model (Building 2 model almost identical)

The periods obtained from eigenvalue analysis of the two models were 0.226 seconds for Building 1 (with all three walls extending to the top) and 0.267 seconds for Building 2 (with two of the three walls extending only up to half the building height), a difference of 18 percent. Clearly the lateral stiffness of Building 2 is less than that of Building 1, since it has a longer fundamental period. Hence, decreasing the wall area in the upper storeys (i.e. Building 2) should reduce the lateral stiffness of a building, and 
therefore lead to a longer fundamental period. This confirms that equation 5.13 is inappropriate when some walls do not extend to the top of the building.

To avoid confusion in the application of equation 5.13, which has been adopted in several international codes (ASCE, 2005; BSSC, 2003; ICC, 2006), it is proposed that all structural walls present at the first storey should be assumed to extend all the way to the top of the building, resulting in the following simplified equation:

$$
A_{e}=\frac{100}{A_{B}} \sum_{i=1}^{N W} \frac{A_{i}}{\left[1+0.83\left(\frac{h_{i}}{D_{i}}\right)^{2}\right]}
$$

\section{Evaluation of Goel and Chopra formula}

Using this simplified equation to determine the equivalent shear area, $A_{e}$, let us now examine how equation 5.12 fits the measured data. Appendix $F$ shows the calculation of $A_{e}$ for a subset of 10 buildings in Montréal whose lateral stiffness in at least one direction is provided by uncoupled shear walls. Table G-1 (Appendix G) shows the measured fundamental periods for this subset of 10 buildings, as well as other information needed to evaluate the Goel and Chopra formula. Table G-2 shows the same information for a subset of buildings in California, adapted from Goel and Chopra (1998). Wall areas from the remaining buildings in data set DS-2 could not be obtained. Note that the wall areas (as percentage of base area) are generally lower for buildings in Montréal than in California, a direct consequence of the lower seismicity in Montréal.

Figure 5.11 shows the ambient data from Montréal buildings, juxtaposed with the data from Goel and Chopra (1998). Also shown are three curves 
representing the best-fit ( $T_{R}$ - thick, middle brown line) to the California data, best-fit minus one standard deviation ( $T_{L}-$ thin, lower black line), and best-fit plus one standard deviation ( $T_{U}$ - thin, upper blue line), adapted from the Goel and Chopra study. The original data used to develop these curves were limited to nine buildings (17 data points), having a maximum ratio $h / A_{e}^{0.5}$ less than $300 \mathrm{~m}$. For this reason, beyond an abscissa value of $300 \mathrm{~m}$, each of these curves is represented by dashed lines to indicate extrapolation from the original data.

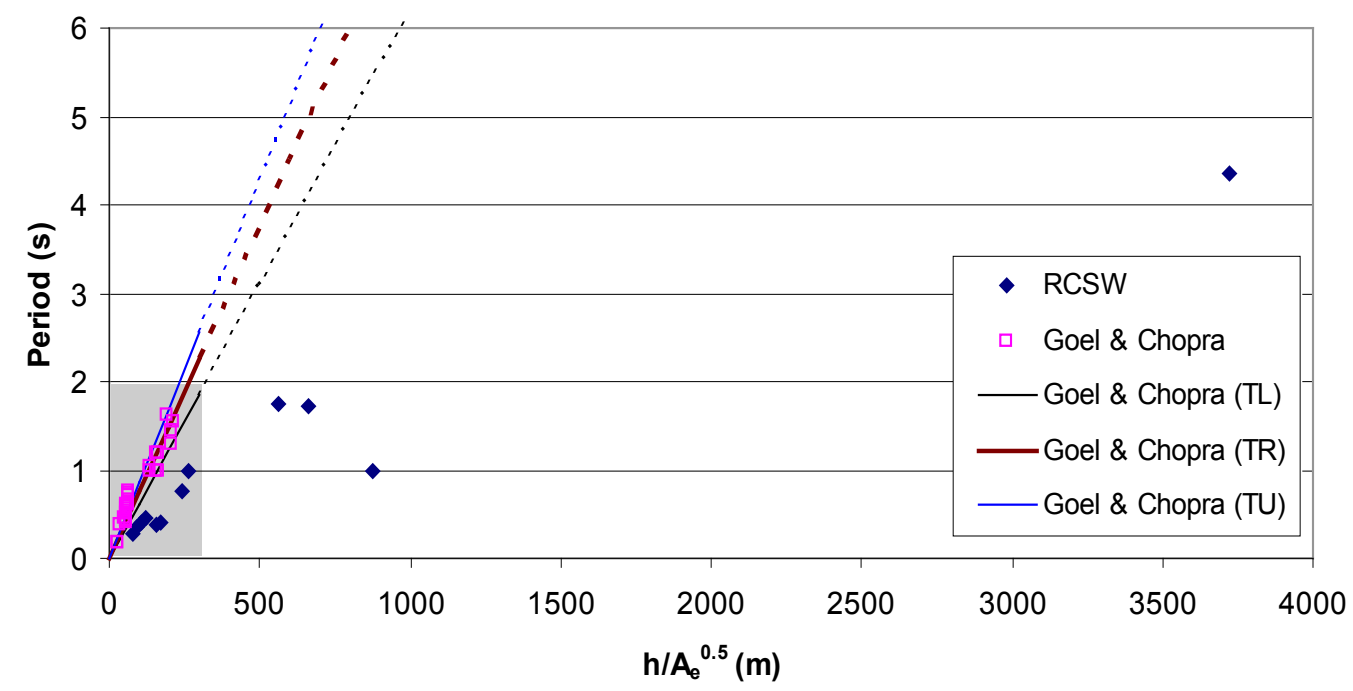

Figure 5.11: Evaluation of Goel and Chopra formula

Notice that four of the twelve data points corresponding to buildings in Montréal have abscissa values larger than $300 \mathrm{~m}$ (RCSW-2, RCSW-13, RCSW-17, and RCSW-20). In fact, for RCSW-2, the ratio $h / A_{e}^{0.5}$ exceeds $3500 \mathrm{~m}$. All of these buildings are at least as tall as the tallest building in the Goel and Chopra database (46 m), and have a total wall area (as percentage of base area) less than the smallest wall area in that same data set. Figure 5.11 clearly shows that the periods of these buildings are significantly shorter than those predicted by the best-fit minus one standard deviation curve. Not surprisingly, this suggests that it is unwise to extrapolate beyond an abscissa value of $300 \mathrm{~m}$. 
Figure 5.12 shows a zoom on the range from 0 to $300 \mathrm{~m}$ (grey zone in Figure 5.11), thereby excluding the data points corresponding to the four buildings mentioned above.

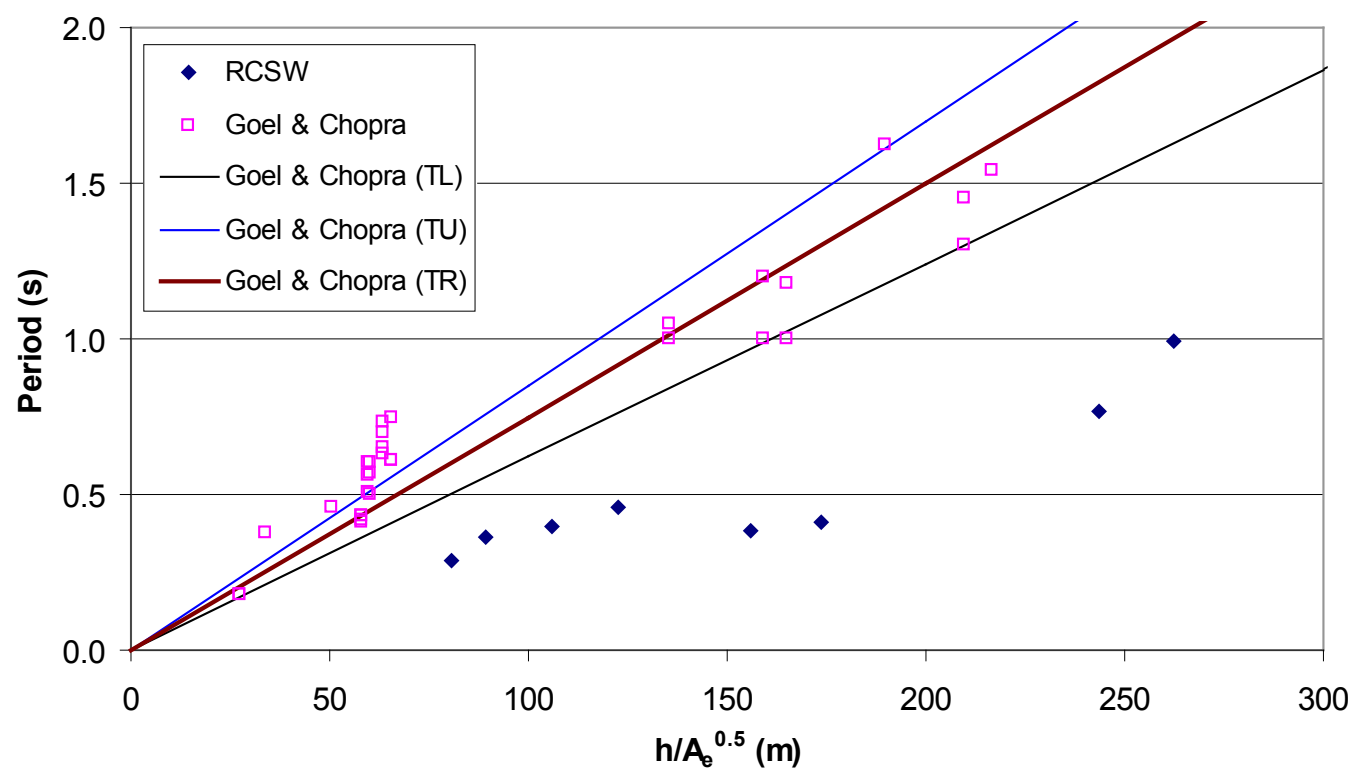

Figure 5.12: Evaluation of Goel and Chopra period formula (zoomed)

Still, the remaining data points for Montréal buildings all lie well below the best-fit minus one standard deviation curve. Though the period is expected to elongate during earthquake ground motions, there is no consensus on the amount of elongation (from ambient to strong motions) that can safely be relied upon. Considering the poor fit of these equations to the measured periods of Montréal buildings, equation 5.12 does not appear to be appropriate to estimate the fundamental period of reinforced concrete shear wall buildings in Eastern Canada.

Further, for large values of $h / A_{e}^{0.5}$, beyond $300 \mathrm{~m}$, the discrepancy between measured and predicted periods is unacceptably large. Currently, design codes allow equation 5.12 to be used for all masonry or concrete shear wall structures. However, Figure 5.11 confirms that it is unwise to 
extrapolate beyond the range of the original data. Therefore, in design codes where equation 5.12 is already suggested, its use should be limited to values of $h / A_{e}^{0.5}$ less than $300 \mathrm{~m}(1000 \mathrm{ft})$.

\subsubsection{Wallace and Moehle formula}

Recall from section 2.3.1.4 that Wallace and Moehle (1992) performed a theoretical analysis of the flexural behaviour of a uniform concrete cantilever of rectangular cross section, supporting regularly distributed floor loads, and having assumed values of floor weight, modulus of elasticity, and storey height, and suggested that the fundamental period of simple, uncracked RCSW buildings could be estimated using

$$
T_{1}=0.0019 \frac{h_{w}}{D} \frac{n}{\sqrt{p}}
$$

In contrast, considering cracked-section stiffness to be equal to half the gross-section stiffness, they suggested

$$
T_{1}=0.0027 \frac{h_{w}}{D} \frac{n}{\sqrt{p}}
$$

to estimate the fundamental period of cracked reinforced concrete shear wall buildings.

According to the authors, periods measured from small amplitude ambient motions should compare well with equation 5.15, while periods from moderate seismic motions should compare well with equation 5.16. Let us examine how well these equations (particularly 5.15) fit the measured period data from Montréal buildings. 
Table G-3 (Appendix G) shows the measured periods of a subset of 10 buildings in Montréal whose lateral stiffness is provided by uncoupled shear walls, as well as the parameters required to estimate the period using equations 5.15 and 5.16. Note that these equations imply that the height and width of all shear walls - in a given direction - are identical. To avoid confusion, all walls were assumed to extend to the top of the building $\left(h_{w}=h\right)$, and the minimum value of $h / D$, corresponding to the largest shear wall, was considered in the period formulae. Similarly, Table G-4 shows results from 10 buildings in Chile and California presented by Wallace and Moehle (1992), most of which were adapted from Midorikawa (1990) and Calcagni Castillo (1988). Notice again that the wall areas are typically smaller in Montréal than in Chile and California.

Figure 5.13 shows the measured periods of 20 reinforced concrete shear wall buildings - 10 in Montréal, 8 in Chile, and 2 in California. Also shown are two curves representing equations 5.15 ( $T_{\text {uncracked }}$ - thick, lower black line) and 5.16 ( $\mathrm{T}_{\text {cracked }}$ - thin, upper brown line). Since most data points are concentrated below an abscissa value of 3000 , Figure 5.14 shows a zoom on the range from 0 to 3000 . 


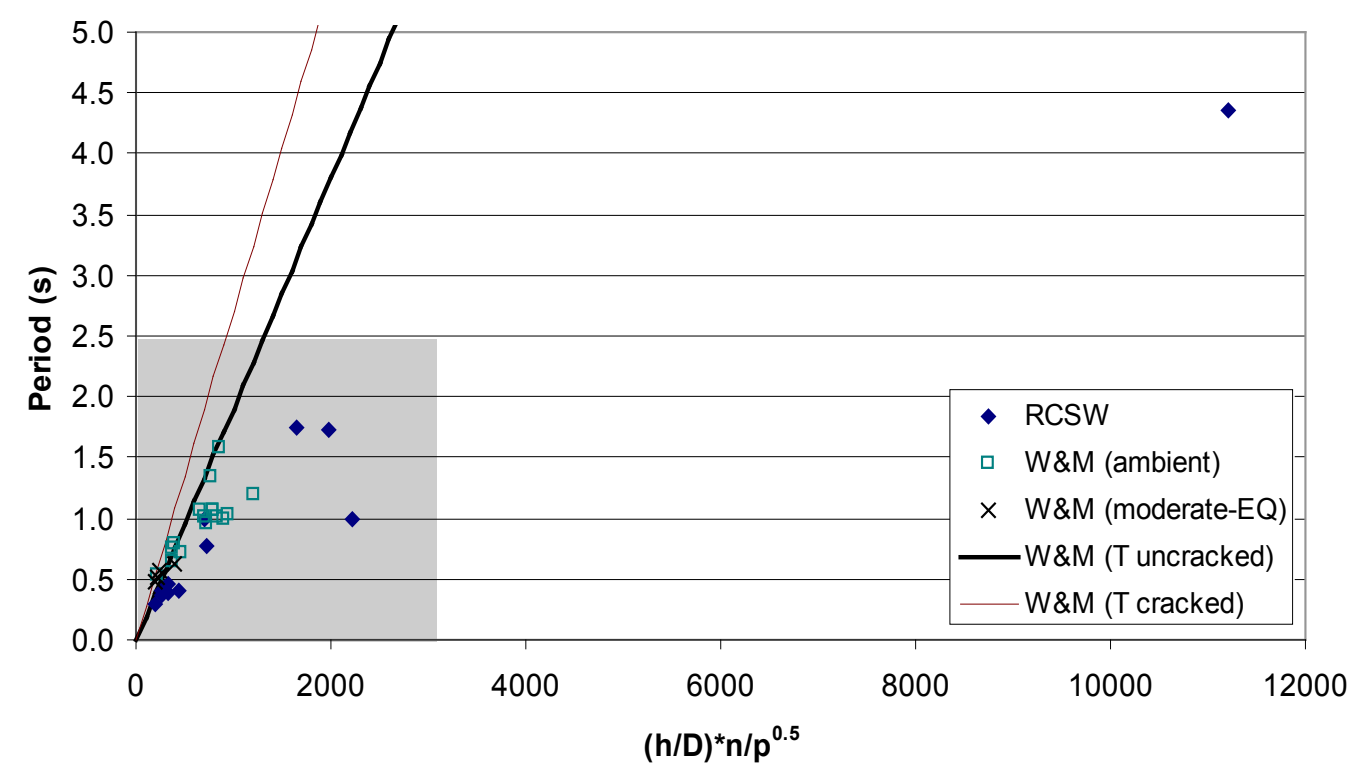

Figure 5.13: Evaluation of Wallace and Moehle period formula

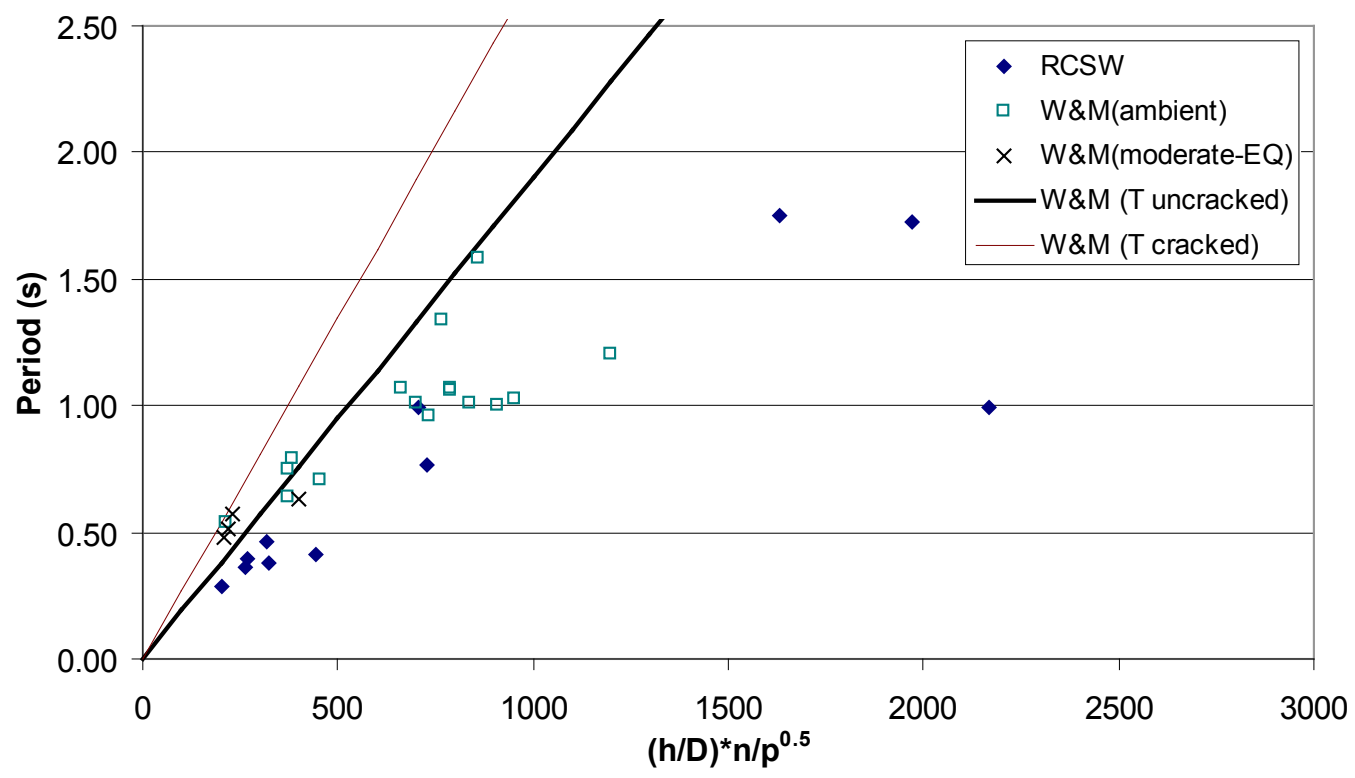

Figure 5.14: Evaluation of Wallace and Moehle period formula (zoomed)

The data points corresponding to all Montréal buildings lie well below the line corresponding to equation 5.15 (uncracked). And again the discrepancy between measured and predicted periods is unacceptably large for large abscissa values. Since these equations tend to 
overestimate the fundamental period, which would lead to unrealistically low values of seismic base shear, they do not appear to be appropriate for the design of buildings in Canada.

\subsubsection{Lee et al formula}

Recall from section 2.3.1.5 that Lee et al (2000) used the fundamental periods of 50 RCSW buildings in Korea, identified from ambient vibration records, to calibrate a theoretical model based on both flexural and shear deformations of a uniform cantilever. The resulting equation is rewritten here for convenience:

$$
T_{1}=0.4\left(\frac{h^{0.2}}{\sqrt{L_{w}}}\right)-0.5
$$

where $L_{w}$ is given by

$$
L_{w}=\frac{\sum_{i=1}^{N W} D_{i}}{A_{B}}
$$

Table G-5 (Appendix G) shows the measured periods of a subset of 10 buildings in Montréal whose lateral stiffness is provided by uncoupled shear walls, as well as the parameters required to estimate the period using equation 5.17. Similarly, Table G-6 shows data from the 50 buildings in Korea used to develop this equation (Lee et al., 2000). Notice again that the values of $L_{w}$ are typically lower for shear wall buildings in Montréal than in Korea, which indicates that smaller wall areas are used in Montréal. 
Figure 5.15 shows the measured periods of 60 reinforced concrete shear wall buildings -10 in Montréal and 50 in Korea. Also shown is a curve representing equation 5.17. As for Figure 5.11, extrapolation beyond the range of the original data is indicated by a dashed line.

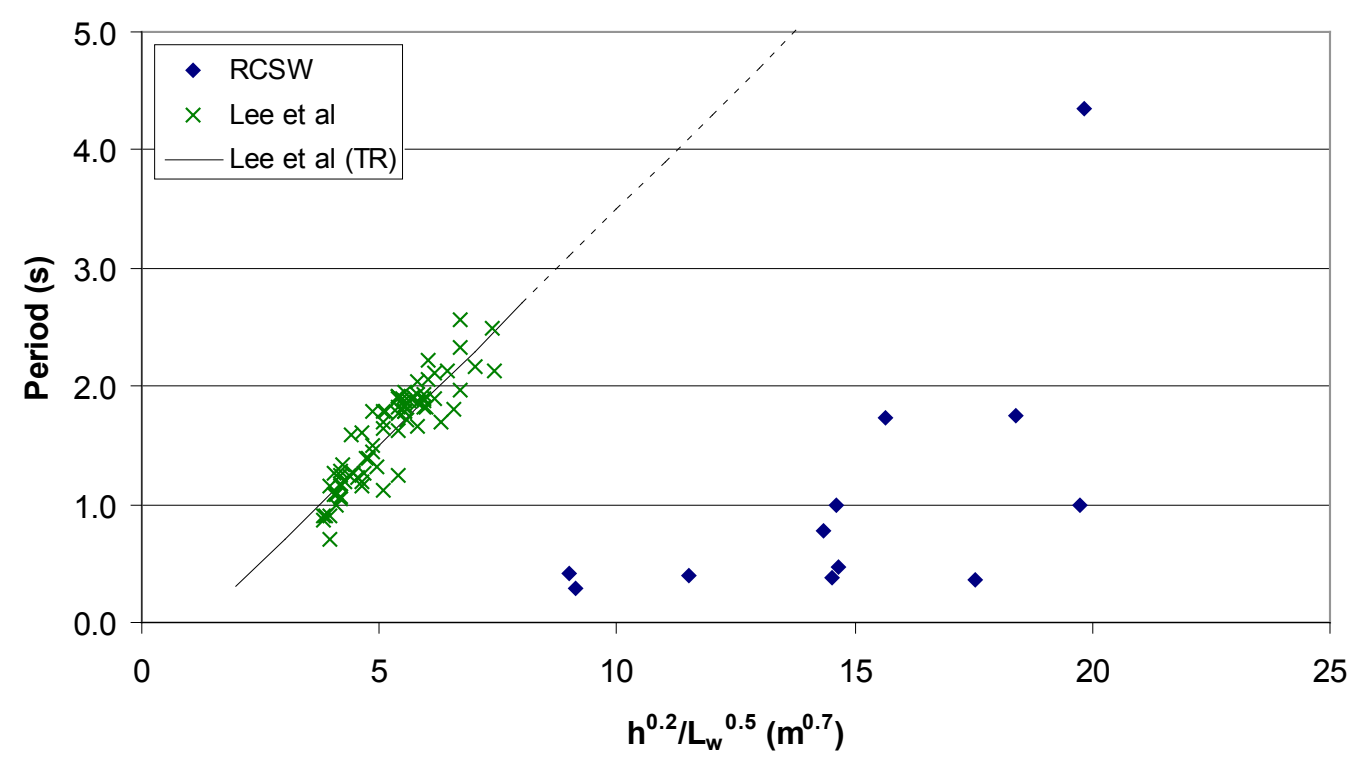

Figure 5.15: Evaluation of Lee et al period formula

Figure 5.15 clearly shows that equation 5.17 is not adequate to predict the fundamental period of concrete shear wall buildings in Montréal. Notice how the Lee et al data are clustered together with only slight variability about the regression line, while the Montréal data are spread out in an almost random fashion, which indicates that the abscissa is not well correlated with the measured periods of Montréal buildings. Further, the fundamental periods of all Montréal buildings are much shorter than the corresponding values from equation 5.17. Therefore, using this equation would lead to unreasonably large fundamental period estimates, which in turn would lead to unrealistically low design seismic loads. 


\subsubsection{Morales formula}

Finally, recall from section 2.3.1.6, that Morales (2000) proposed a model in which the fundamental periods of RCSW buildings depend on both the height and the second moment of area of the building, and which was calibrated using the measured fundamental periods of 18 RCSW buildings in California. The resulting equation is again rewritten here for convenience:

$$
T_{1}=0.13 \frac{h}{I^{0.25}}-0.4
$$

Table G-7 (Appendix G) shows the measured periods of a subset of 10 buildings in Montréal whose lateral stiffness is provided by uncoupled shear walls, as well as the parameters required in equation 5.19. Similarly, Table G-8 shows data from the 18 buildings in California used to develop this equation. Figure 5.16 shows the measured periods from both studies plotted against the ratio $h / I^{0.25}$. Again, extrapolation beyond the range of the original data is indicated by a dashed line.

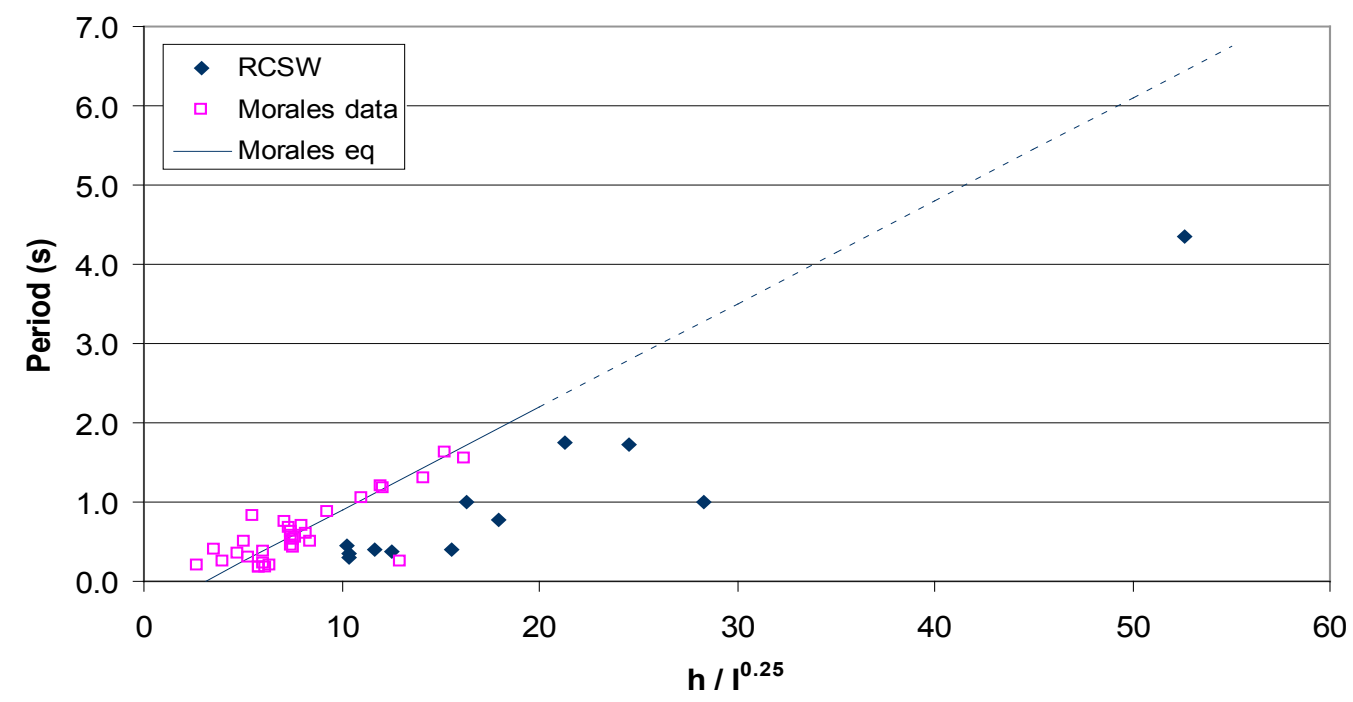

Figure 5.16: Evaluation of Morales period formula 
As for all the previous equations, the data points corresponding to all Montréal buildings lie below the curve. In fact, many of the measured periods are more than three times shorter than those predicted by equation 5.19. Let us reiterate that the period is expected to elongate during strong ground motions, but it would appear unsafe to rely on that much period elongation for design purposes. It should be noted that, unlike for the previous equations, the discrepancy between measured and predicted periods is not uncharacteristically large for large abscissa values.

Though this equation seems to be a slight improvement over the previous equations, considering the additional data from buildings in Montréal, it still does not adequately predict the fundamental period of concrete shear wall buildings.

\subsubsection{Improved fundamental period formulae}

As we have seen, the equation currently adopted in several building codes to estimate the fundamental period of reinforced concrete shear wall buildings is not very accurate, and, despite requiring a priori knowledge of the dimensions of all shear walls, the alternative equations studied do not improve the estimation of the period. In contrast, a model in which the fundamental period varies linearly with the building height was found to improve the fit to the measured period data for both data sets considered.

Considering this, it is suggested that the mean value of the fundamental period of reinforced concrete shear wall buildings should be predicted using the following equation:

$$
T_{1}=0.020 h
$$


This equation is similar to what has previously been suggested for similar buildings in France (Farsi and Bard, 2004) and Italy (Lagomarsino, 1993). Note that the linear form of this equation can be obtained by Rayleigh's method using an identical set of assumptions to that used in developing the current code equation, only with the base shear being proportional to $T^{-1}$ rather than $T^{2 / 3}$ (Goel and Chopra, 1997). Interestingly, this is in agreement with the design response spectrum in ASCE 7-05, which varies in this fashion for a wide range of building periods (ASCE, 2005, p.115).

However, to calculate design seismic loads using acceleration spectra, the fundamental period should generally be underestimated, thus leading to conservative loads. Rather than use the mean value, it has been suggested to use the mean minus one standard deviation curve (Goel and Chopra, 1998). Based on available data, there is a 16 percent probability that the fundamental period of a given building will fall below this curve. The mean minus one standard deviation curve for each data set was obtained by lowering the best-fit line, in logarithmic format, by the standard error of estimate without changing its slope:

$$
\alpha_{L}=\alpha_{R}-s_{e}
$$

where subscript $L$ (lower) indicates the value of $\alpha$ corresponding to the mean minus one standard deviation, and subscript $R$ (regression) indicates the value of $\alpha$ corresponding to the best-fit. The coefficient of the period equation was then computed from

$$
a_{L}=\exp \left(\alpha_{L}\right)
$$


In contrast, since displacement spectra from seismic ground motions typically increase with period, seismic displacements may be underestimated when an unrealistically low fundamental period is used. For the purposes of calculating seismic displacements, it is therefore recommended to use the mean plus one standard deviation curve (Chopra and Goel, 2000). This curve was obtained for each data set by raising the best-fit line by the standard error of estimate, without changing its slope, as follows:

$$
\alpha_{U}=\alpha_{R}+s_{e}
$$

where subscript $U$ (upper) indicates the value of $\alpha$ corresponding to the best-fit plus one standard deviation. The coefficient of the period equation was then computed using

$$
a_{U}=\exp \left(\alpha_{U}\right)
$$

Table 5.3 shows the equations corresponding to the mean (best-fit), mean minus one standard deviation (best-fit $-1 \sigma$ ), and mean plus one standard deviation (best-fit $+1 \sigma$ ) obtained from regression analysis for the two data sets considered. Based on these data, roughly 16 percent of RCSW buildings in Eastern Canada can be expected to have fundamental periods shorter than the mean minus one standard deviation curve, and a further 16 percent can be expected to have periods longer than the best-fit plus one standard deviation curve.

Table 5.3: Best-fit, best-fit $-1 \sigma$, and best-fit $+1 \sigma$ equations obtained from regression analyses

\begin{tabular}{|c|c|c|c|c|c|}
\hline & & \multicolumn{3}{|c|}{ Equation } & \\
\cline { 3 - 5 } Data set & $\alpha_{\mathbf{R}}$ & Best-fit & Best-fit $-1 \sigma$ & Best-fit $+1 \boldsymbol{\sigma}$ & $\mathbf{s}_{\mathbf{e}}$ \\
\hline DS-1 & -3.9666 & $T_{1}=0.019 h$ & $T_{1}=0.015 \mathrm{~h}$ & $T_{1}=0.024 \mathrm{~h}$ & 0.230 \\
\hline DS-2 & -3.9379 & $T_{1}=0.020 \mathrm{~h}$ & $T_{1}=0.013 \mathrm{~h}$ & $T_{1}=0.030 \mathrm{~h}$ & 0.431 \\
\hline
\end{tabular}


In light of these results, to calculate design base shear, the following equation is suggested to predict the fundamental period of concrete shear wall buildings in Eastern Canada:

$$
T_{1}=0.015 h
$$

On the other hand, to calculate seismic displacements, the fundamental period should instead be estimated using

$$
T_{1}=0.025 h
$$

Further, equation 5.26 could be used to define the maximum period that can be used in the equivalent static force procedure of the NBCC. Recall from section 2.2.3 that, in calculating seismic design loads for an RCSW building using the ESFP, the maximum period allowed by the code is twice that calculated from equation 5.1. In contrast, for moment-resisting frame buildings, the code only permits a deviation of 1.5 from the appropriate code equation. Interestingly, the period calculated from equation 5.26 is 1.67 times longer than that calculated from equation 5.25. Hence, if equation 5.25 was adopted to replace the current NBCC equation for RCSW buildings (equation 5.1), then the maximum period could be limited to about 1.5 times that calculated from equation 5.25 , as for momentresisting frame buildings.

Note that equations $5.20,5.25$, and 5.26 were calibrated using measured data from buildings having heights lower than $270 \mathrm{~m}$; hence, extrapolating these relationships beyond a height of $270 \mathrm{~m}$ is not recommended. Figures 5.17 and 5.18 show how these equations compare to the periods from data sets DS-1 and DS-2. 


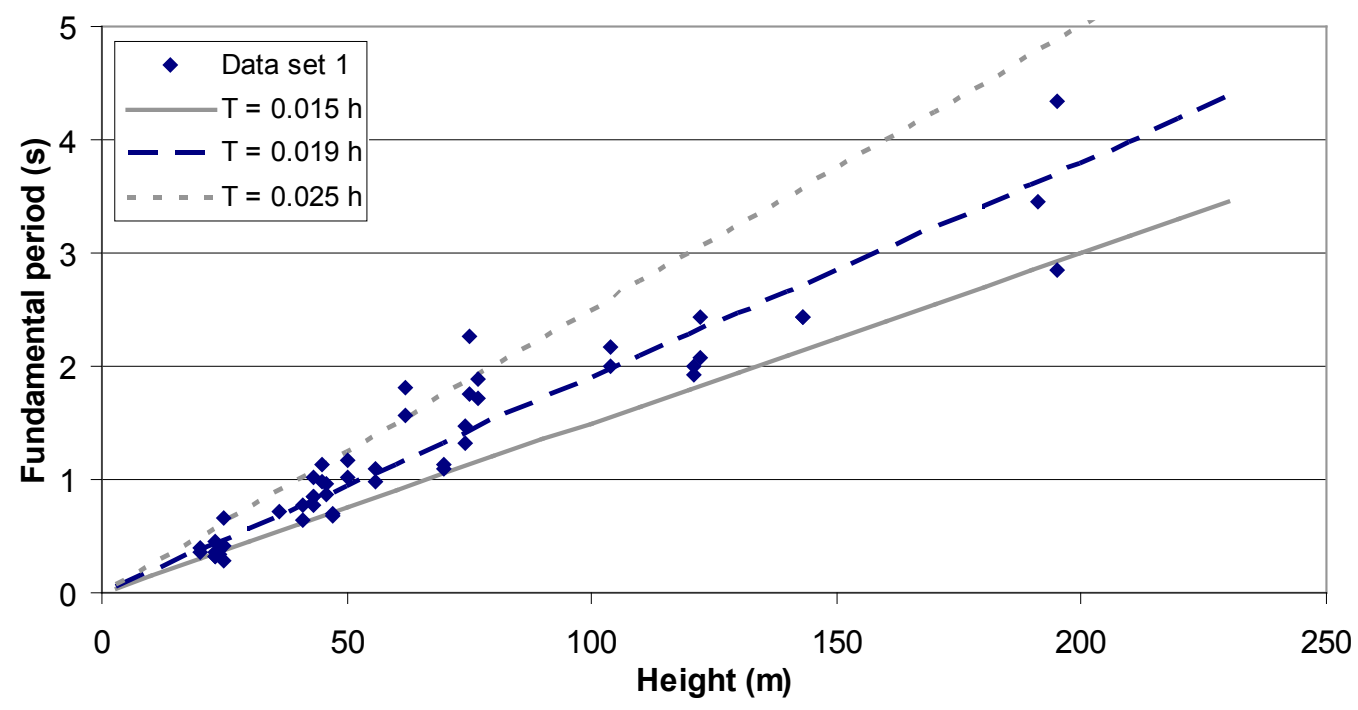

Figure 5.17: Comparison of improved period formulae for RCSW buildings with DS1

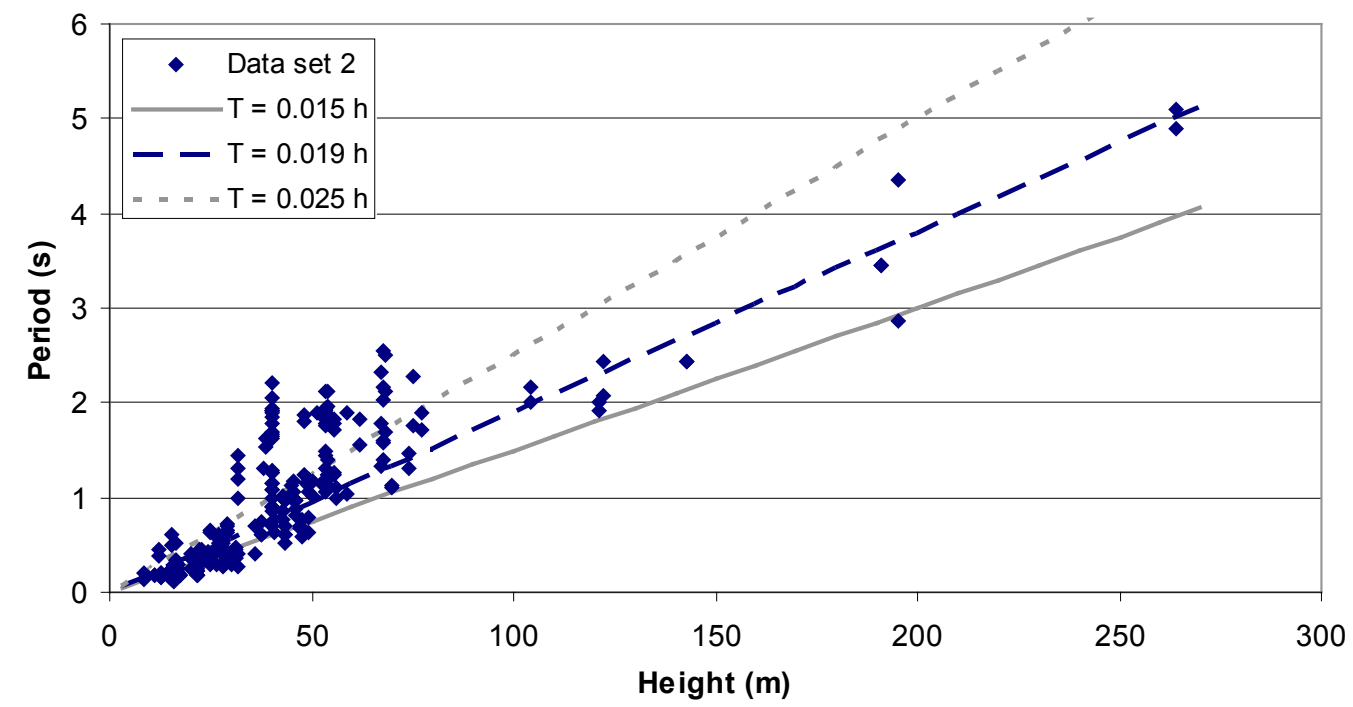

Figure 5.18: Comparison of improved period formulae for RCSW buildings with DS2

Finally, note that equations 5.20, 5.25, and 5.26 were developed considering mainly period data from low-amplitude ambient vibrations. As mentioned in section 2.3.1.7, the fundamental period is expected to elongate during strong ground shaking. However there is still no consensus in the earthquake engineering community regarding the 
sources and magnitude of this period elongation. As a result, it is not currently possible to reliably quantify the period elongation that can be expected from low-amplitude vibrations to strong ground motions. Therefore, it may not be prudent to rely on this elongation for the purposes of calculating lateral seismic loads until the phenomenon is better understood. On the other hand, when calculating seismic displacements, it may be advisable to take account of some additional period elongation.

\subsection{Damping}

As we have seen, the capacity of a building to dissipate energy during vibration is vital to reducing the vibration amplitudes during dynamic loading events, such as strong winds or earthquake ground motions. In structural analysis, simple damping models, such as Rayleigh damping or constant modal viscous damping, are typically used due to their mathematical convenience, rather than a rigorous understanding of the underlying phenomena.

As discussed in section 2.3.2, in seismic analysis, five percent viscous damping is typically assumed for all low frequency vibration modes and design spectra in building codes are typically specified for five percent damped oscillators. To determine the dynamic effects of wind, lower damping values are generally recommended, as damping is expected to increase with the amplitude of building motion.

Let us now use the measured damping values of RCSW buildings in Montréal to evaluate these assumptions. Further, the results are used to examine whether any of the models presented in section 2.3.2 improve the prediction of structural damping in various modes of vibration. 


\subsubsection{Measured damping in RCSW buildings in Montréal}

The low-amplitude damping ratios of up to six vibration modes were identified for the 27 reinforced concrete shear wall buildings studied and are presented in Appendix C. These are plotted in Figure 5.19 against building height. Clearly, the data are quite scattered; therefore, assuming the same amount of damping in all vibration modes of all RCSW buildings is not very accurate. Most of the measured damping ratios are concentrated between 1 and 4 percent of critical. Note that the mean of the 107 damping values is 2.2 percent, while the median is nearer to 2 percent, with 53 of the 107 damping values being smaller than 2 percent. Furthermore, only 18 damping values (or roughly 17 percent) are smaller than 1.5 percent.

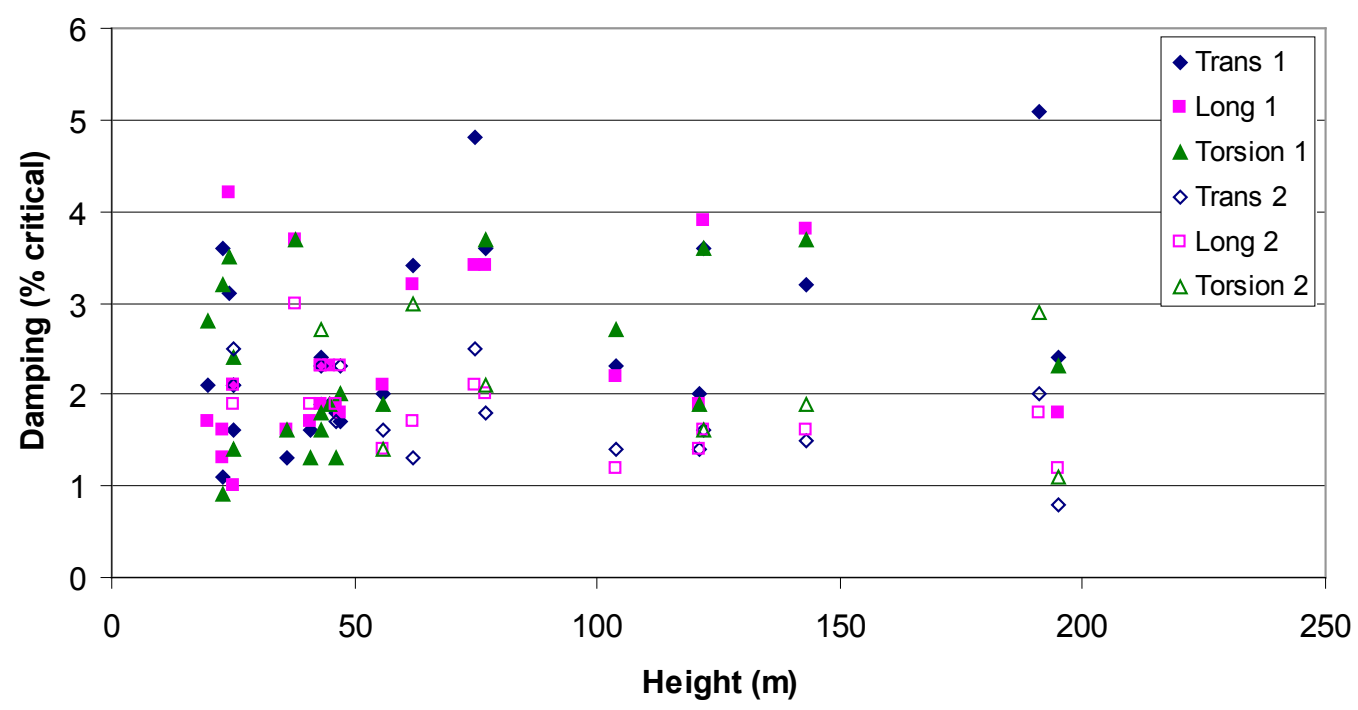

Figure 5.19: Measured damping ratios of first six vibration modes of RCSW buildings in Montréal

Let us now examine whether the alternative models discussed above can be used to improve the prediction of damping in RCSW buildings.

\subsubsection{Damping in fundamental translation modes}


Though the correlations were weak, damping in the fundamental translation mode has been reported to be correlated with building height, number of storeys, and natural frequency. Note that some of the models discussed in section 2.3.2 have an amplitude-dependent term (equations 2.52 and 2.56). Since the damping data considered here were obtained from ambient vibration tests, these are assumed to represent lowamplitude damping ratios. For this reason, in the following discussion, the amplitude-dependent terms were neglected.

Figure 5.20 shows the measured damping ratios of the fundamental translation modes of the $27 \mathrm{RCSW}$ buildings plotted against building height. Also shown is the curve corresponding to equation 2.54, suggested by Satake et al (2003). Clearly, the data are quite scattered, and there is no apparent correlation between damping and building height. Particularly telling is the fact that, at a height of approximately 25 metres, damping was found to vary by a factor of four.

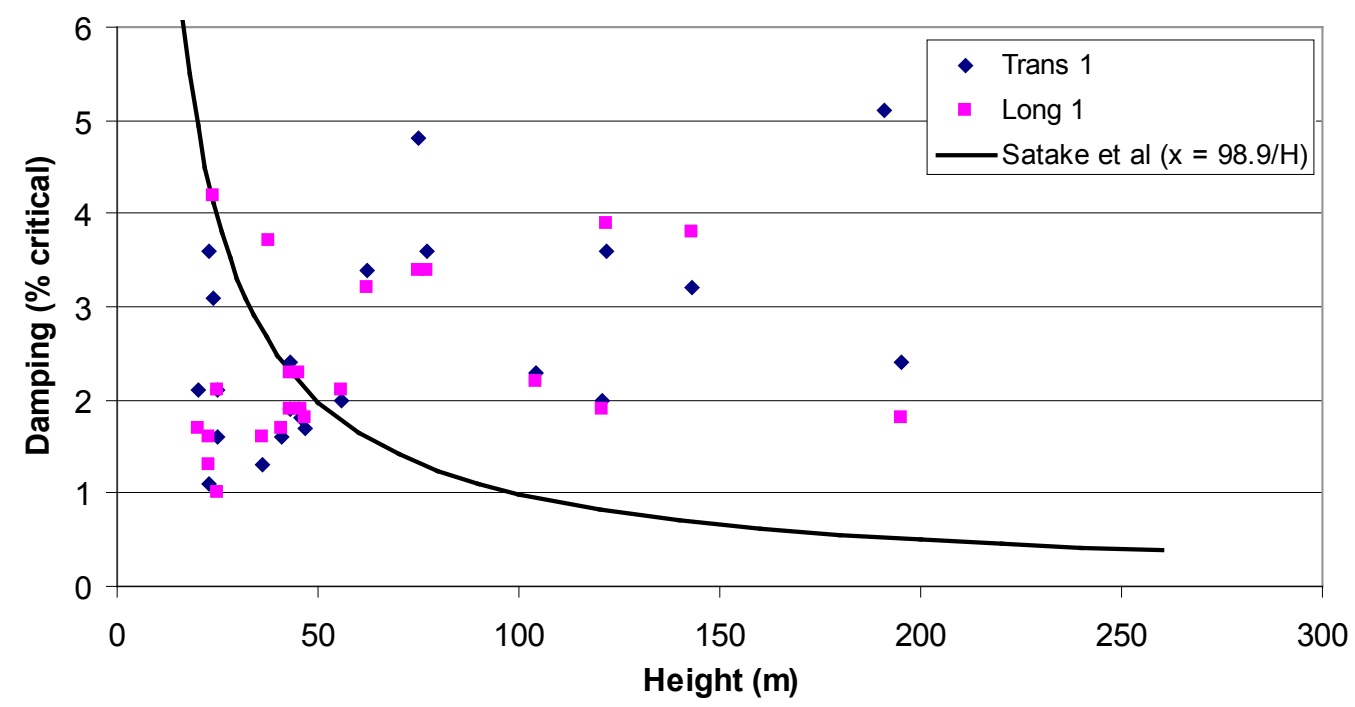

Figure 5.20: Correlation of damping in fundamental translation mode with building height for RCSW buildings in Montréal 
Figure 5.21 shows the same data plotted against the number of storeys. Also shown is the curve corresponding to equation 2.59, developed by Fritz et al (2009). Again, the data are quite scattered, and the correlation between damping and the number of storeys is very weak.

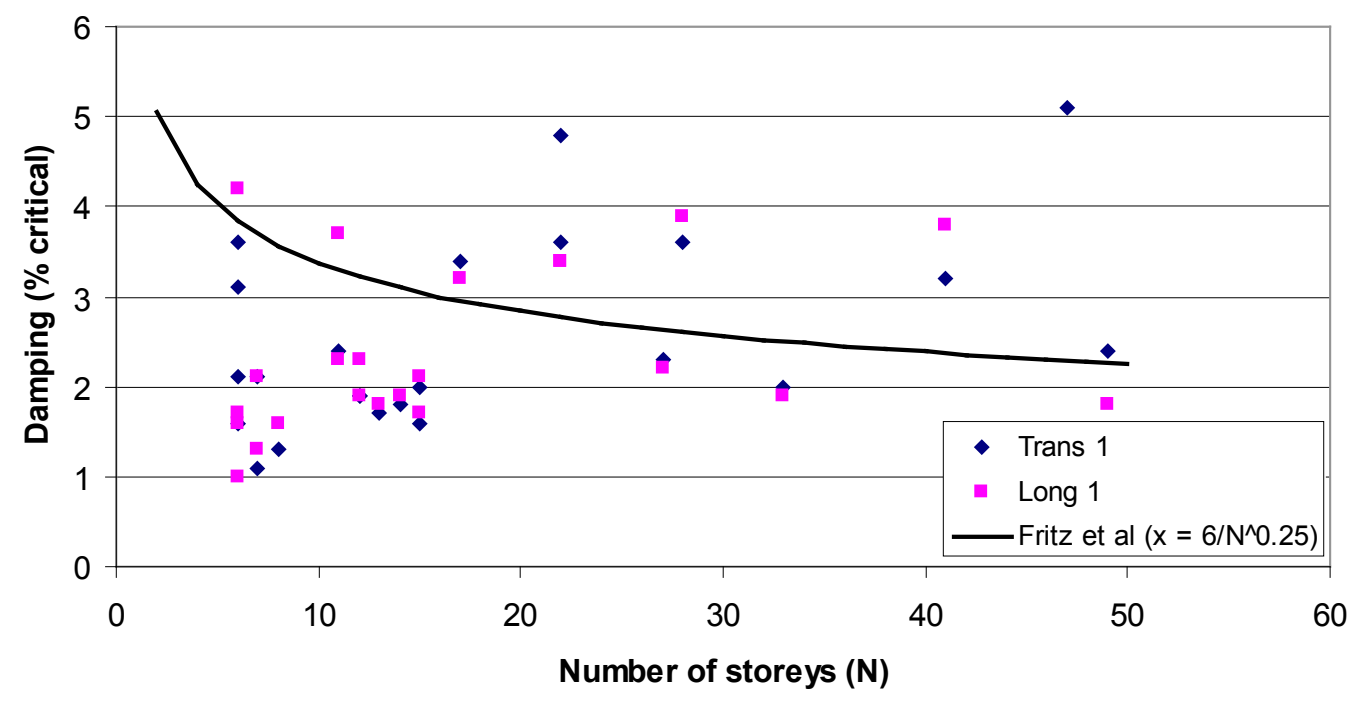

Figure 5.21: Correlation of damping in fundamental translation mode with number of storeys for RCSW buildings in Montréal

Finally, Figure 5.22 shows the same data plotted against the natural frequency of the corresponding mode. Also shown are the curves corresponding to the models suggested by Jeary (1986) (solid line), Lagomarsino (1993) (dashed line), and Satake et al (dotted line). Here, there does appear to be a correlation between damping and natural frequency, at least below a frequency of approximately $2 \mathrm{~Hz}$, with damping values generally decreasing with increasing frequency. Interestingly, this runs contrary to the models proposed by Jeary and Satake et al, in which damping increases with frequency, and the recommendations of the CTBUH, which suggest that damping in tall (low frequency) buildings is generally lower than in shorter (higher frequency) buildings. 
In contrast, the model proposed by Lagomarsino fits the measured damping data relatively well at frequencies below $2 \mathrm{~Hz}$, slightly underestimating damping in most cases. However, beyond a frequency of $2 \mathrm{~Hz}$, the model predicts that damping increases with frequency. Though only a few data points are available for frequencies above $2 \mathrm{~Hz}$, many of these fall below the dashed line. Further, damping values at very low frequencies may be unreasonably large. Therefore, for very tall buildings (with fundamental frequencies lower than approximately $0.2 \mathrm{~Hz}$ ), damping values should not be obtained using this model.

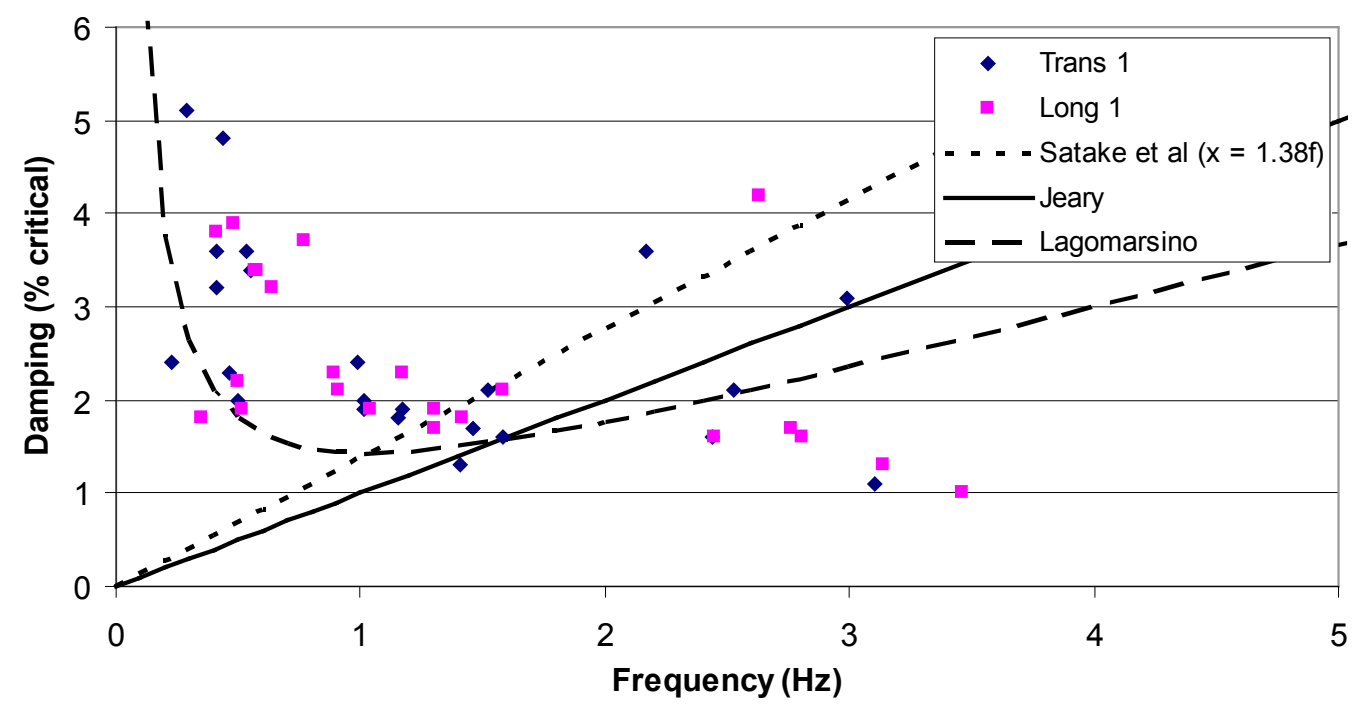

Figure 5.22: Correlation of damping in fundamental translation mode with fundamental frequency for RCSW buildings in Montréal

As we have just seen, damping in the fundamental translation mode of RCSW buildings does not appear to be correlated with the building height nor the number of storeys. Though damping and natural frequency appear to be weakly correlated, the observed trend - that damping seems to decrease with increasing frequency - conflicts with the findings of Jeary and Satake et al. The model proposed by Lagomarsino fits the measured damping values fairly well for frequencies less than $2 \mathrm{~Hz}$, but may overestimate damping at higher frequencies, as well as at very low 
frequencies. In light of these findings, it is clear that none of the above models can be reliably used to predict damping in the fundamental translation modes. It would seem unwise to rely on increased damping in low frequency (tall) buildings, as the data from this study suggest, since this conflicts with the findings of Jeary and Satake et al. It would however seem equally unwise to rely on increased damping in high frequency (short) buildings, as implied by equations 2.52 and 2.55 .

\subsubsection{Damping in fundamental torsion mode}

For reinforced concrete buildings, Satake et al suggested a linear relationship between damping in the fundamental torsion mode and the corresponding natural frequency. Let us examine whether damping in the fundamental torsion mode of RCSW buildings in Montréal is correlated with the corresponding frequency, or with building height. Figure 5.23 shows the measured damping data for the fundamental torsion mode of RCSW buildings in Montréal. Again, there appears to be no correlation between damping and frequency for the fundamental torsion mode of RCSW buildings. If anything, damping appears to decrease with increasing frequency, which is again in contradiction with the findings of Satake et al. Similarly, no significant correlation between damping and building height for the fundamental torsion mode was observed. 


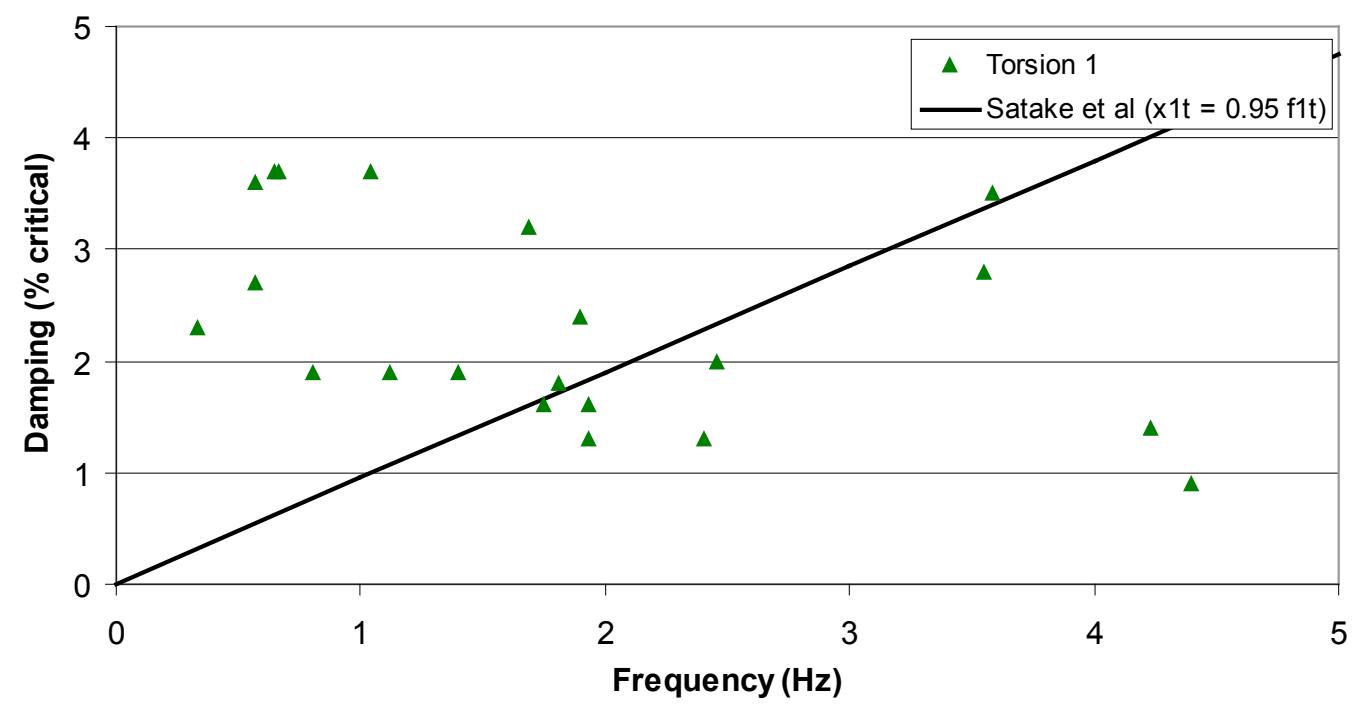

Figure 5.23: Correlation of damping in fundamental torsion mode with frequency for RCSW buildings in Montréal

\subsubsection{Damping in higher modes}

The model proposed by Lagomarsino was meant to apply to all translational vibration modes of a building. Figure 5.24 shows the measured damping values for the first six (lowest frequency) vibration modes of the $27 \mathrm{RCSW}$ buildings in this study. As for the fundamental vibration mode, the Lagomarsino model fits the measured data reasonably well for frequencies below approximately 2 or $3 \mathrm{~Hz}$. But, at very low frequencies and beyond a frequency of $3 \mathrm{~Hz}$, this model appears to overestimate damping in most cases. 


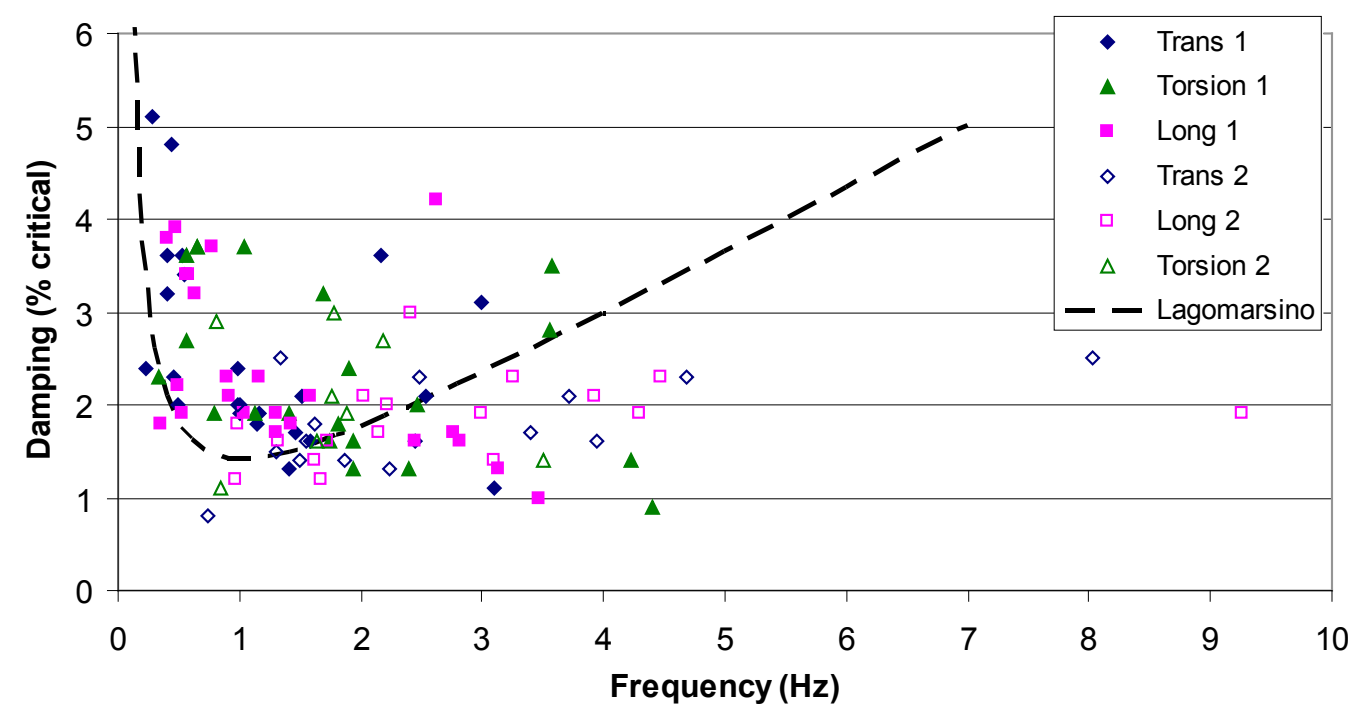

Figure 5.24: Correlation of damping in first six vibration modes with natural frequency for RCSW buildings in Montréal

In contrast, Satake et al suggested that damping in higher modes tended to increase with the mode number, with the damping in each mode being approximately 1.4 times larger than the previous (lower frequency) mode in the same direction. Figure 5.25 shows the measured damping ratios of the second vibration modes in each direction plotted against the damping ratios of the corresponding fundamental modes. Also shown are curves corresponding to the recommendations of Satake et al, as well as a 45 degree line representing equal damping in successive vibration modes. The data do not seem to support the conclusions of Satake et al. In fact, most data points fall below the equal damping line, indicating that the second mode damping ratios are generally lower than those for the corresponding first mode. 


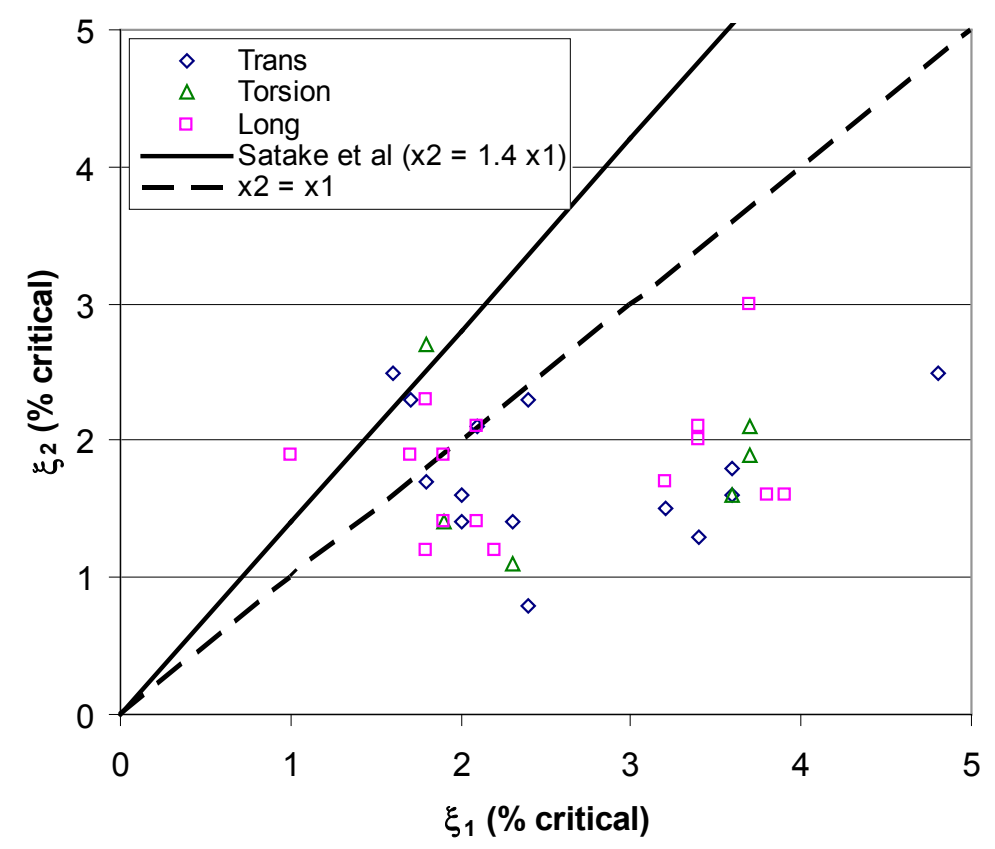

Figure 5.25: Second mode vs fundamental mode damping for RCSW buildings in Montréal

\subsubsection{Suggested damping values for RCSW buildings}

The above discussion shows that the recorded damping data from RCSW buildings in Montréal do not follow the same trends that have been reported by other researchers for similar buildings. Though this may be the result of differences in design and construction techniques, it seems more likely that it is the result of the variability in damping values that has always afflicted damping data. As mentioned above, the damping models presented here were developed based on relatively weak correlations, and the results of this study suggest that, when a different data set is considered, the relationships no longer hold.

Therefore, it appears that robust models that more accurately reflect the various mechanisms of energy dissipation in vibrating buildings are required to improve the prediction of damping. Further, as discussed in section 4.1.4, measured damping values are typically prone to significant 
uncertainty due to the limitations of modal identification methods, which may also contribute to the difficulties in developing robust models that produce damping estimates which compare well to measured damping data from real buildings.

In the foreseeable future, simple methods will continue to be used to account for damping in structural analysis and design. Since damping attenuates the amplitude of a structure's dynamic response, it is important not to overestimate this parameter.

As we have seen, most of the damping values of reinforced concrete shear wall buildings identified in this study were in the range of 1 to 4 percent of critical. For wind applications, the National Building Code of Canada (NRC/IRC, 2005b) currently suggests the use of 2 percent critical viscous damping. The data from this study suggest that this represents roughly the mean amount of damping that can be expected during relatively low-amplitude motions. It would be safer to assume 1.5 percent damping for reinforced concrete shear wall buildings, since only 17 percent of damping values identified in this study fell below this value. However, this is not judged to be a critical priority since the difference in building response between 1.5 and 2 percent damping is not likely to be significant in design.

In the case of earthquake ground motions, Fritz et al (2009) showed that, on average, damping can be expected to double from ambient to earthquake motions. Recall however that energy dissipation due to hysteretic (or inelastic) response of structural members is typically accounted for in structural analysis by using hysteretic material models (in nonlinear dynamic analysis) or ductility-related force modification factors (in linear methods). It is important to note that "damping" refers to the energy dissipation capacity of a building that is not explicitly modeled in 
the analysis. For this reason, energy dissipation due to inelastic response must not be used to further increase damping. Unfortunately, it is not clear whether the earthquake data considered by Fritz et al involved any buildings that experienced significant inelastic structural response, in which case the damping values used may have included some hysteretic energy dissipation.

Nevertheless, accepting their findings, damping of 3 percent of critical would seem appropriate for all vibration modes of RCSW buildings during design-level seismic ground motions. This is higher than what is recommended by the Council on Tall Buildings and Urban Habitat for buildings between 50 and $250 \mathrm{~m}$ tall. However, it is lower than the 5 percent that is typically assumed in seismic analysis. Indeed, in most modern building codes, including the 2005 edition of the National Building Code of Canada, seismic design spectra are specified for 5 percent damped oscillators. Though it is possible to adjust these spectra for different levels of damping (Atkinson and Pierre, 2004), practicing engineers are not likely to do so if it is not explicitly required. For these reasons, it is suggested that in future, design spectra be specified at lower levels of damping, or, acceptable methods to adjust design spectra for different levels of damping be explicitly required.

\subsection{Natural periods of torsion and second translation modes}

As mentioned previously, dynamic analysis methods typically require the estimation of the natural periods of torsion and higher vibration modes, in addition to the fundamental periods. However, little guidance is provided to help engineers ensure that the estimated values are reasonable, as compared to the measured periods of these vibration modes in similar buildings. 
In section 2.3.3, we introduced a few simple models that have been proposed by Lagomarsino (1993) and Satake et al (2003) to estimate the natural periods of the fundamental torsion and second translation modes. These models are evaluated here using the measured periods of 27 RCSW buildings in Montréal. The data used for this purpose are presented in Appendix C. Further, the correlations of the second torsion period with both building height and the fundamental torsion period are also studied.

\subsubsection{Period of fundamental torsion mode}

Since the period of the fundamental torsion mode, $T_{l t}$, has been related to both the fundamental translation period and the building height in previous studies, regression analyses were performed considering each of these variables in turn. These regression analyses were performed identically to those for the fundamental period (see section 5.1.5). The candidate models were all of the form

$$
T_{1 t}=a z^{b}
$$

where $a$ and $b$ were the parameters to be obtained from the regression analyses, and $z$ was either the fundamental translation period, $T_{1}$, or the building height. The equation was recast into linear form by taking the natural logarithm of each side, as follows:

$$
y=\alpha+b x
$$

where $y=\ln \left(T_{1 t}\right), \alpha=\ln (a)$, and $x=\ln (z)$. Again, visual inspection of the data in logarithmic format confirmed that it was reasonable to assume 
equal variance of $y$ for all values of $x$. Standard linear regression techniques were then used considering the data in logarithmic format. The results of the regression analyses are summarized in Table 5.4.

Table 5.4: Results of regression analyses for fundamental torsion period of RCSW buildings in Montréal

\begin{tabular}{|c|c|c|c|}
\hline Type & Best fit & $\mathbf{S}_{\mathbf{e}}$ & $\mathbf{R}^{\mathbf{2}}$ \\
\hline Unconstrained (height) & $T_{1 t}=0.013 \mathrm{~h}^{1.029}$ & 0.329 & 0.825 \\
\hline Constrained (height) & $T_{1 t}=0.015 \mathrm{~h}$ & 0.330 & 0.816 \\
\hline Unconstrained $\left(\mathrm{T}_{1}\right)$ & $T_{1 t}=0.780 T_{1}^{0.995}$ & 0.262 & 0.886 \\
\hline Constrained $\left(\mathrm{T}_{1}\right)$ & $T_{1 t}=0.780 T_{1}$ & 0.262 & 0.887 \\
\hline
\end{tabular}

First, an unconstrained regression analysis was performed considering building height as the independent variable. The equation thus obtained was nearly linear with respect to building height; thus, an additional analysis was carried out, constraining the exponent $b$ to unity. Table 5.4 shows that, as expected, the fit for the constrained regression was slightly inferior, but still quite good. Figure 5.26 shows the measured fundamental torsion periods of the 27 buildings studied, as well as the best-fit for the constrained regression analysis. To illustrate the variability in the data, Figure 5.26 also shows the best-fit minus one standard deviation and bestfit plus one standard deviation curves, which were derived as described in section 5.1.7. 


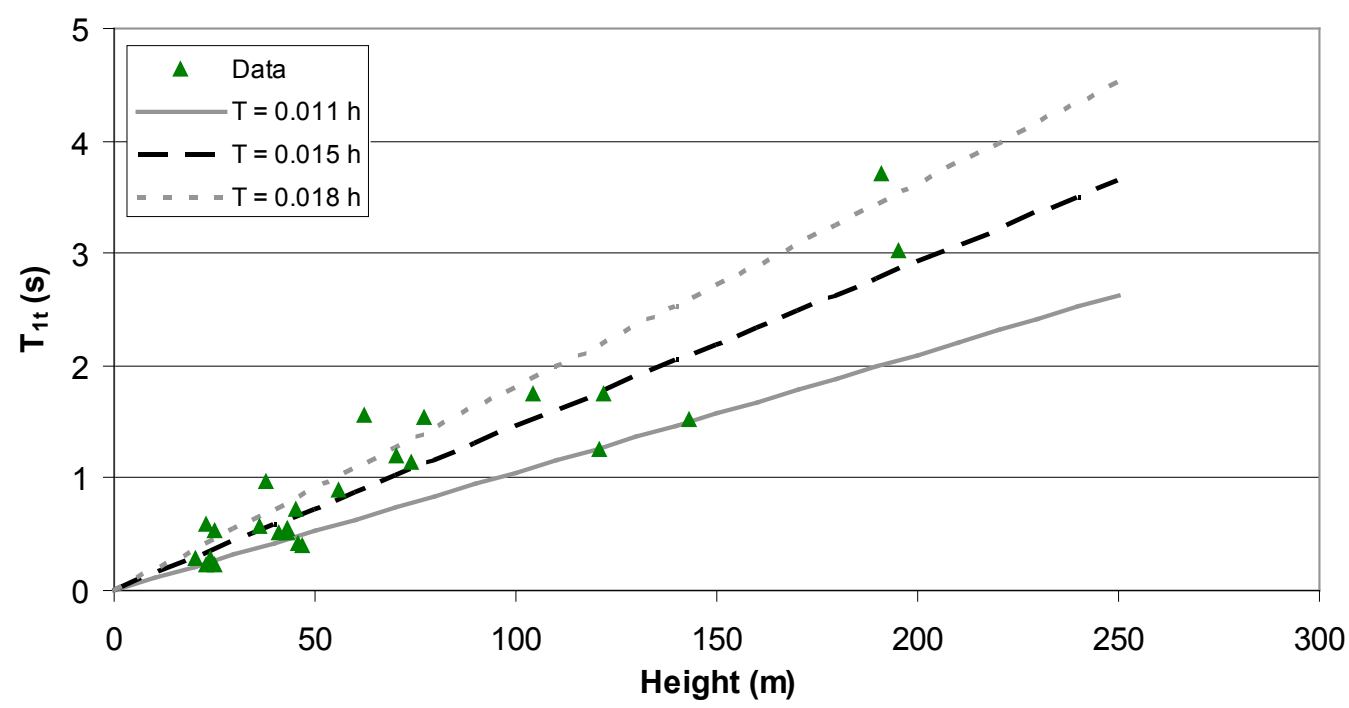

Figure 5.26: Correlation of fundamental torsion period with building height for RCSW buildings in Montréal

Clearly, there is a significant correlation between building height and $T_{1 t}$. Therefore, the mean of the period of the fundamental torsion mode of RCSW buildings can be estimated reasonably well using

$$
T_{1 t}=0.015 \mathrm{~h}
$$

This equation is very similar to equation 2.60 , suggested by Lagomarsino. Interestingly, it is also quite similar to the best-fit from the constrained regression analysis on the fundamental translation period.

Next, a similar analysis was performed considering the fundamental translation period, $T_{1}$, as the independent variable, instead of the building height. Since each building has two fundamental translation periods - one in each principal direction - two data points were considered for each torsion period; that is, each torsion period was paired separately with each of its corresponding translation periods. Again, the equation corresponding to the best-fit was nearly linear, so an additional analysis was performed constraining the exponent $b$ to unity. Figure 5.27 shows the measured fundamental torsion periods, plotted against $T_{1}$ for the 27 buildings 
studied. Also shown are the curves corresponding to the best-fit from the constrained regression analysis, the best-fit minus one standard deviation, and the best-fit plus one standard deviation.

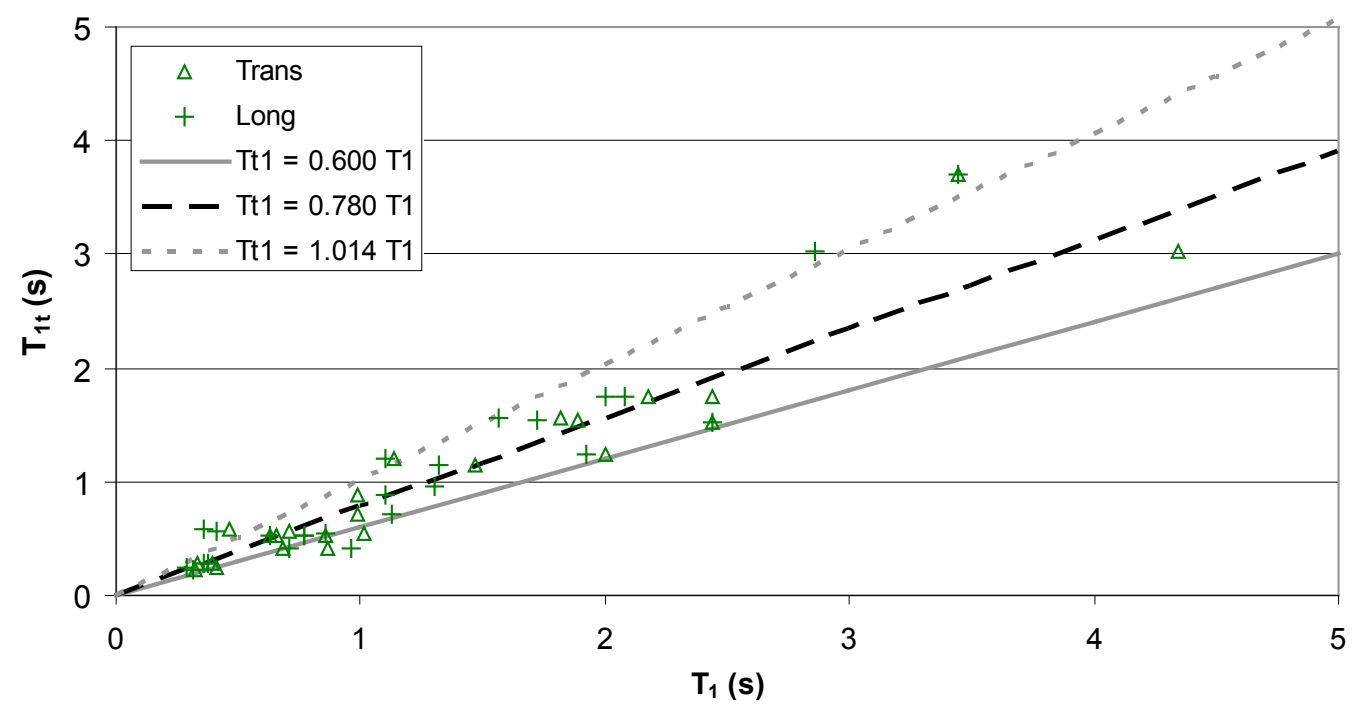

Figure 5.27: Correlation of fundamental torsion period with fundamental translation period for RCSW buildings in Montréal

Hence, the mean period of the fundamental torsion mode can be estimated reasonably accurately using

$$
T_{1 t}=0.78 T_{1}
$$

This equation agrees remarkably well with the recommendations of Satake et al. (equation 2.61).

Note that the regressions based on the fundamental translation period are superior to those based on building height, as shown by the lower $s_{e}$ and higher $R^{2}$ values in Table 5.4. This suggests that $T_{l t}$ can be estimated more accurately by equation 5.30 than by equation 5.29 . However, this is only the case if the fundamental translation period is known a priori. In most cases where one might be interested in predicting the fundamental 
torsion period of a building, the fundamental translation period is not likely to be known either. One notable exception is if measurements were performed in a building, allowing the identification of fundamental translation periods, but not that of the fundamental torsion mode. In such a case, it would be appropriate to estimate $T_{1 t}$ using equation 5.30. Further, every building has two fundamental translation periods: obtaining a single estimate of $T_{1 t}$ from two values of $T_{1}$ could therefore cause some confusion. In contrast, building height should be known at all stages of the design process.

Bearing in mind these practical considerations, in most cases, equation 5.29 should be used to obtain an estimate of the fundamental torsion period of RCSW buildings. Note however that most of the buildings used to develop equations 5.29 and 5.30 had shear walls concentrated in the central core. These equations are meant to provide an estimate of the frequency of the fundamental torsion mode, but they should be used with care, particularly in buildings in which shear walls are positioned further apart, which may be stiffer in torsion than predicted by the above equations.

\subsubsection{Period of second translation modes}

Based on the theoretical behaviour of flexural and shear cantilevers, the periods of the second translation modes (one in each principal direction), $T_{2}$, have been related to their corresponding fundamental periods in past studies. Thus, regression analyses were again performed considering the fundamental period as the independent variable. However, as mentioned for the fundamental torsion mode, in most cases where one might be interested in estimating $T_{2}$, the fundamental periods are not likely to be known. Therefore, relationships based on the fundamental period are of limited use. For this reason, regression analyses were also performed 
considering building height as the independent variable. The results of the regression analyses are summarized in Table 5.5.

Table 5.5: Results of regression analyses for second translation period of RCSW buildings in Montréal

\begin{tabular}{|c|c|c|c|}
\hline Type & Best-fit & $\mathbf{s}_{\mathbf{e}}$ & $\mathbf{R}^{\mathbf{2}}$ \\
\hline Unconstrained (height) & $T_{2}=0.010 h^{0.875}$ & 0.245 & 0.836 \\
\hline Constrained (height) & $T_{2}=0.009 h^{0.9}$ & 0.245 & 0.843 \\
\hline Unconstrained $\left(T_{1}\right)$ & $T_{2}=0.321 T_{1}^{0.924}$ & 0.119 & 0.961 \\
\hline Constrained $\left(T_{1}\right)$ & $T_{2}=0.314 T_{1}$ & 0.128 & 0.961 \\
\hline
\end{tabular}

First, an unconstrained regression based on building height was carried out. Table 5.5 shows that the fit was quite good, as illustrated by the high value of the coefficient of determination. The exponent was then rounded to the first decimal place and an unconstrained regression was performed. Figure 5.28 shows the second translation periods of RCSW buildings plotted against building height for the 27 buildings studied. Also shown are the curves corresponding to the best-fit, best-fit minus one standard deviation, and best-fit plus one standard deviation, from the constrained regression. 


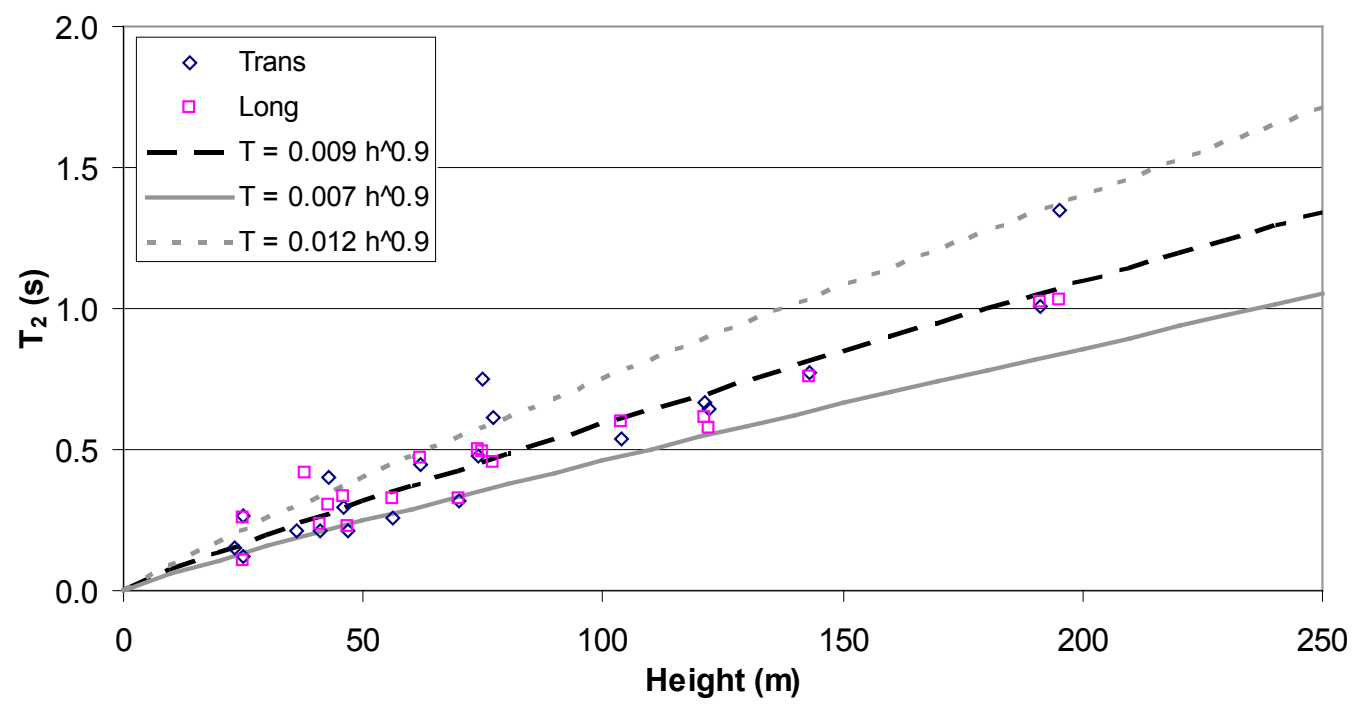

Figure 5.28: Correlation between second translation period and building height for RCSW buildings

Figure 5.28 clearly shows that there exists a strong correlation between building height and $T_{2}$. The mean of the periods of the second translation modes can therefore be estimated using

$$
T_{2}=0.009 h^{0.9}
$$

A similar procedure was followed considering the fundamental period, $T_{1}$, as the independent variable. The best-fit from the unconstrained regression analysis fit the data remarkably well, with a coefficient of determination of 0.96 . In contrast to the regression based on building height, rather than rounding the exponent to the first decimal place (0.9), it was rounded up to unity, and a constrained regression was performed. Though this approach led to a slightly worse fit, it allowed direct comparison with the findings from previous research. Figure 5.29 shows the second translation mode periods of RCSW buildings plotted against the corresponding fundamental periods for the same buildings, as well as the curves representing the best-fit, best-fit minus one standard deviation, 
and best-fit plus one standard deviation from the unconstrained regression.

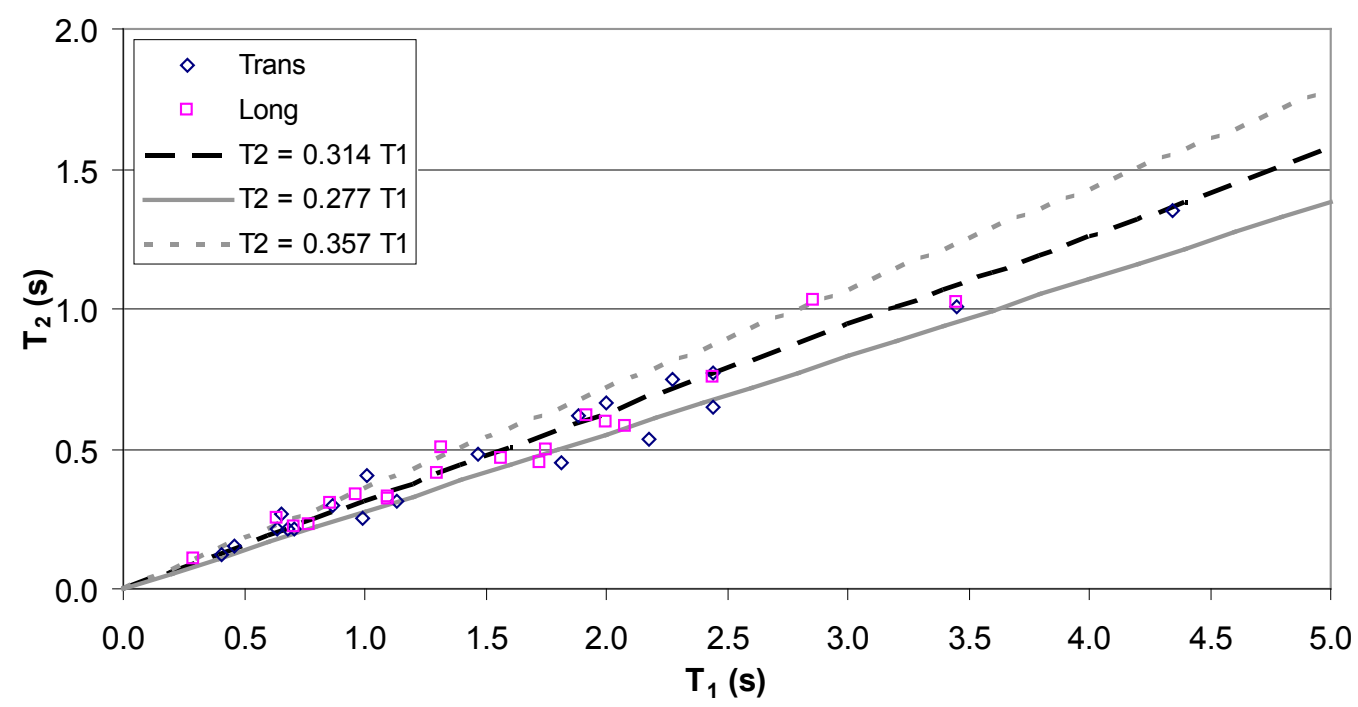

Figure 5.29: Correlation between second translation period and corresponding fundamental period for RCSW buildings

Again, the strong correlation between $T_{2}$ and $T_{1}$ is evident. Alternatively to equation 5.31, the mean periods of the second translation modes of RCSW buildings can be estimated from the corresponding fundamental periods using

$$
T_{2}=0.31 T_{1}
$$

Note that the coefficient of 0.31 agrees remarkably well with those proposed by Satake et al (0.31) and Lagomarsino (0.27). Further, it is also closer to that of a shear cantilever (0.33) than that of a flexural cantilever (0.16). This seems to suggest that the shear building model generally used to represent the dynamic behaviour of buildings (see section 2.1.6) is indeed representative of the expected behaviour of RCSW buildings, at least with respect to the ratio of higher to lower mode vibration periods. 
Note also that the quality of the fit of equation 5.32 , based on $T_{1}$, is superior to that of equation 5.31, based on building height. Hence, in cases where the fundamental translation periods are known, the second translation mode periods of RCSW buildings should be estimated using equation 5.32. The rest of the time, it would seem more appropriate to use equation 5.31 .

\subsubsection{Period of second torsion mode}

As mentioned previously, little work has been done regarding the period of the second torsion mode of buildings. Considering the results for the other vibration modes discussed thus far, it is reasonable to expect that the second torsion period, $T_{2 t}$, should be correlated with both the building height and the fundamental torsion period. Though the second torsion period could only be identified for 13 of the 27 RCSW buildings considered in this study, these data nevertheless provide the opportunity to study these relationships. Regression analyses were again carried out considering in turn the building height and the fundamental torsion period as the independent variable. Table 5.6 shows the results of these analyses.

Table 5.6: Results of regression analyses for second torsion period of RCSW buildings in Montréal

\begin{tabular}{|c|c|c|c|}
\hline Type & Best-fit & $\mathbf{S}_{\mathbf{e}}$ & $\mathbf{R}^{\mathbf{2}}$ \\
\hline Unconstrained (height) & $T_{2 t}=0.007 h^{0.960}$ & 0.339 & 0.812 \\
\hline Constrained (height) & $T_{2 t}=0.006 h$ & 0.340 & 0.823 \\
\hline Unconstrained $\left(T_{1 t}\right)$ & $T_{2 t}=0.383 T_{1 t}^{0.931}$ & 0.282 & 0.870 \\
\hline Constrained $\left(T_{1 t}\right)$ & $T_{2 t}=0.381 T_{1 t}$ & 0.287 & 0.882 \\
\hline
\end{tabular}

These results show that an unconstrained regression based on building height fit the measured data reasonably well, yielding a coefficient of determination of 0.81 . The best-fit equation was nearly linear, so a second 
regression was performed, constraining the exponent to unity. The fit was slightly affected, as reflected by the slightly higher value of the standard error of estimate. Figure 5.30 shows the second torsion periods of the subset of 13 RCSW buildings plotted against building height, as well as the curves corresponding to the best-fit, best-fit minus one standard deviation, and best-fit plus one standard deviation for the constrained regression.

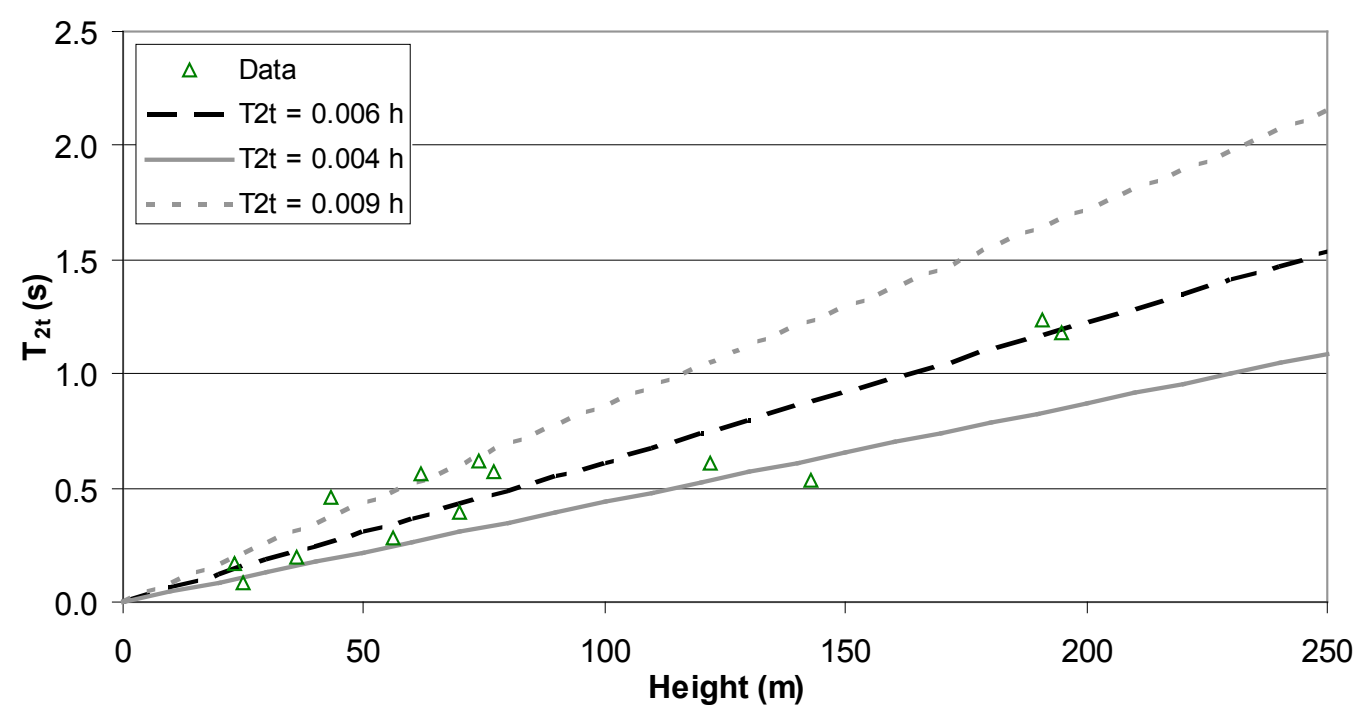

Figure 5.30: Correlation between second torsion period and building height for RCSW buildings

As for the periods of the other vibration modes, the period of the second torsion mode, $T_{2 t}$, is clearly correlated to the building height, and can be estimated using

$$
T_{2 t}=0.006 h
$$

The unconstrained regression based on the fundamental torsion period improved the fit slightly, as illustrated by the higher value of the coefficient of determination, yielding again an equation that was nearly linear. Hence, another regression was carried out by constraining the exponent to unity. 
Figure 5.31 shows $T_{2 t}$ for the subset of 13 RCSW buildings plotted now against the fundamental torsion mode, $T_{1 t}$. Also shown are the curves corresponding to the best-fit, best-fit minus one standard deviation, and best-fit plus one standard deviation for the constrained regression.

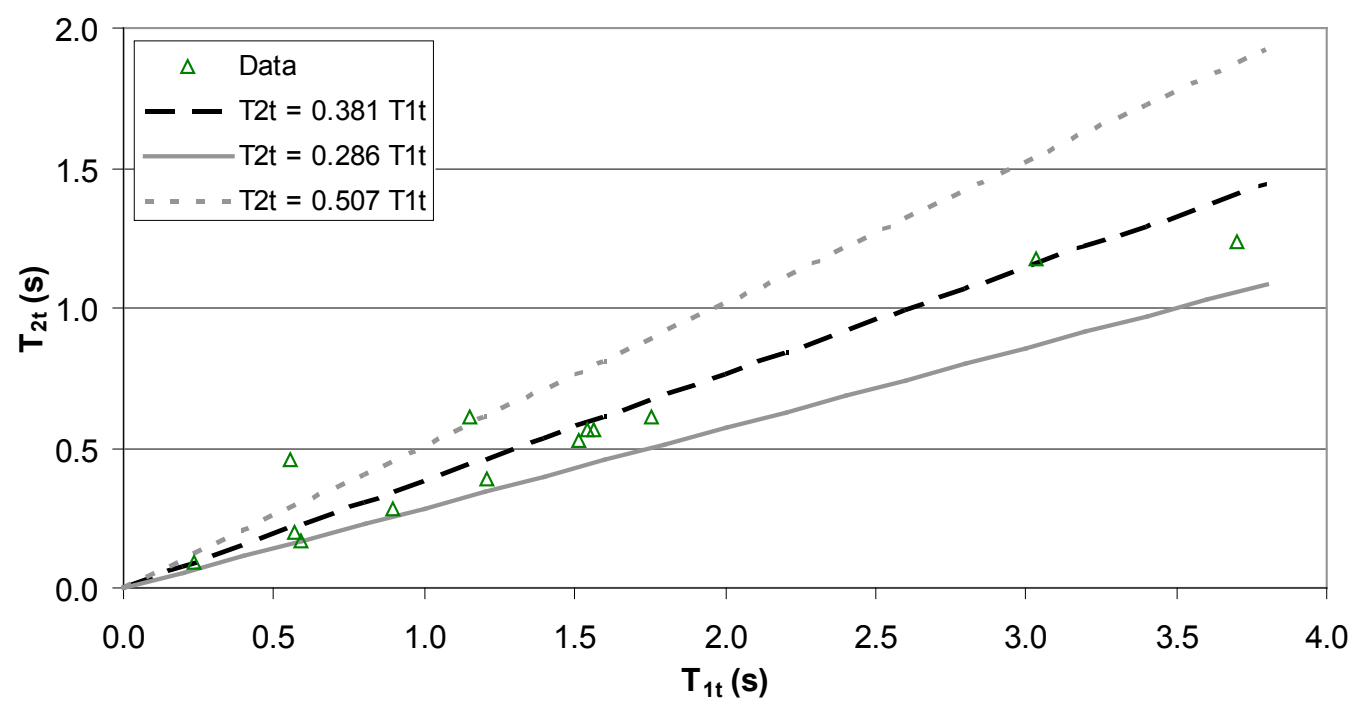

Figure 5.31: Correlation between second torsion period and fundamental torsion period for RCSW buildings

As for the second translation modes, the quality of the fit was slightly improved by considering an equation based on $T_{1 t}$, rather than building height. Thus, when the fundamental torsion period is known, but not the second torsion period, the latter can be estimated from the former using

$$
T_{2 t}=0.29 T_{1 t}
$$

For the reasons discussed previously, in situations where the fundamental torsion period is not known, it would seem more appropriate to estimate $T_{2 t}$ using equation 5.33. And, as mentioned in section 5.3.1, equations 5.33 and 5.34 may not be representative of buildings in which shear walls are positioned far apart. 


\section{Conclusions and recommendations}

The main objectives of this thesis were to examine how the models and assumptions commonly used to estimate the dynamic properties of reinforced concrete shear wall (RCSW) buildings compared to those measured in real buildings, and to determine whether different models could be developed to better represent the dynamic properties of these types of buildings. To this end, the dynamic properties of $27 \mathrm{RCSW}$ buildings on the island of Montréal were identified from their recorded ambient motions.

The results of regression analyses showed that the equation proposed in the 2005 edition of the National Building Code of Canada (NBCC 2005) to estimate the fundamental period of RCSW buildings could be improved. Further, damping in these buildings was shown to vary considerably, confirming that the values commonly assumed in design may not always be conservative. Finally, simple models to predict the natural periods of torsion and second translation modes in RCSW buildings were developed. These findings are summarized below.

This thesis should help engineers verify that the dynamic properties of RCSW buildings assumed in design are realistic and could lead to improvements in building code recommendations. Ultimately, this should lead to improved simulation of the dynamic response of RCSW buildings.

\subsection{Fundamental period of RCSW buildings}

The measured fundamental periods of 27 RCSW buildings in Montréal (DS-1), in addition to those of 130 similar buildings obtained from available literature (DS-2), were compared to those predicted using the equation 
currently adopted in several building codes, including the NBCC 2005. Using independent regression analyses on each data set, the code equation was found to fit the measured data rather poorly and the fits were considerably improved by using a linear model relating the fundamental period and building height.

Alternative equations proposed by Goel and Chopra (1998), Wallace and Moehle (1992), Lee et al (2000), and Morales (2000) to predict the fundamental periods of RCSW buildings using shear wall dimensions, in addition to building geometry, were also evaluated. Despite requiring a priori knowledge of shear wall dimensions, none of these adequately reflected the measured fundamental periods of RCSW buildings in Montréal.

Based on these results, an improved equation to predict the mean fundamental periods of RCSW buildings was proposed. Further, to provide a measure of the uncertainty associated with these predictions, equations corresponding to the mean minus one standard deviation, and mean plus one standard deviation were also produced. These are given by

$$
\begin{aligned}
& \text { Mean: } \quad T_{1}=0.020 \mathrm{~h} \\
& \text { Mean }-1 \sigma: T_{1}=0.015 h \\
& \text { Mean }+1 \sigma: T_{1}=0.025 h
\end{aligned}
$$

in which $h$ is in metres and $T_{l}$ is in seconds.

Since acceleration spectra used to calculate design seismic forces generally decrease with increasing fundamental periods, the equations proposed in codes generally aim to underestimate the fundamental periods, thus leading to conservative estimates of seismic forces. In this 
spirit, it is suggested that the equation corresponding to the mean minus one standard deviation (equation 6.2) be used to estimate fundamental periods for the purpose of calculating seismic forces using equivalent static methods. Based on the data considered in this study, only about one sixth of RCSW buildings should have fundamental periods lower than those predicted using this equation. This equation would, in the author's opinion, be a significant improvement over the equation included in the NBCC 2005.

In contrast, since displacement spectra tend to increase with increasing fundamental periods, the fundamental period should not be underestimated when calculating seismic displacements. Typically, designers will perform design checks on lateral building displacements using the fundamental period calculated from a structural model, as allowed by the code; however it may be advisable to specify that this period should be at least as long as that calculated using the mean plus one standard deviation equation (equation 6.3). Further, this equation could also be used to define the maximum period that can be used to calculate seismic design forces using equivalent static methods.

Furthermore, an inconsistency in the Goel and Chopra formula for buildings in which certain shear walls do not extend to the top of the building was highlighted and a simplified equation to calculate the equivalent shear area $\left(A_{e}\right)$ was proposed. Further, this formula was found to significantly overestimate the fundamental periods of Montréal buildings that had large values of the variable $h / A_{e}$, beyond the range used to develop the formula. Since this formula is adopted in several codes as an alternative method to estimate the fundamental periods of buildings with shear walls, it may be advisable to revise the equation proposed in these codes to compute the equivalent shear area and limit the use of this formula to values of $h / A_{e}$ less than $300 \mathrm{~m}$ (or $1000 \mathrm{ft}$ ). 


\subsection{Damping in RCSW buildings}

The measured damping values in the first six (lowest frequency) vibration modes of $27 \mathrm{RCSW}$ buildings in Montréal were shown to be scattered between 1 and 5 percent of critical viscous damping, with most values lying between 1 and 4 percent. The mean value of these 107 damping values was 2 percent, while roughly one sixth of damping values were lower than 1.5 percent critical. This variability suggests that assuming equal damping in all vibration modes of all RCSW buildings is not very accurate, which is not surprising since the viscous damping model does not capture the complex mechanisms of energy dissipation in real buildings.

Further, damping was not found to be correlated to building height, the number of storeys, nor the natural frequency of the corresponding mode, for any of the vibration modes considered. If anything, the observed trends conflicted with those observed by other researchers for similar buildings. Though this could be the result of regional variations in building construction, it appears more likely that it results from the large variability in recorded damping data. Hence, the weak trends that can be observed on a given data set break down when a different data set is considered.

Since none of the damping models studied here considerably reduce the variability in measured damping values, it may be wise to adopt conservative damping values for design purposes. For the effects of wind, the NBCC 2005 recommends the use of 2 percent critical damping, which seems appropriate for RCSW buildings based on the values observed in this study. For extra conservatism, a value of 1.5 percent would be appropriate, especially when checking serviceability requirements, since only roughly one sixth of buildings in this study had damping values 
smaller than this. Considering the findings of Fritz et al (2009) that a twofold increase in damping can be expected, on average, from ambient to strong motions, it may be justified to use larger damping values for seismic design. Damping values of 3 to 4 percent critical would appear to be appropriate. The value of 5 percent critical that is often assumed in design, and that is used in uniform hazard spectra, seems to be unconservative. For code purposes, it may therefore be prudent to develop design spectra for lower damping values, or to specify acceptable methods to adjust these spectra for different damping levels.

\subsection{Natural periods of torsion and second translation modes}

As suggested in previous studies, the natural periods of the fundamental torsion mode $\left(T_{1 t}\right)$ and the second translation modes $\left(T_{2}\right)$ were shown to be correlated to both building height $(h)$ and the fundamental translation period $\left(T_{1}\right)$ for the RCSW buildings in this study. Similarly, the period of the second torsion mode $\left(T_{2 t}\right)$ was shown to be correlated to both building height and the fundamental torsion period. Based on regression analyses using the measured periods of $27 \mathrm{RCSW}$ buildings in Montréal, equations were proposed to predict the mean natural periods of these vibration modes. Further, to provide a measure of the uncertainty associated with each of these predictions, equations corresponding to the mean minus one standard deviation and mean plus one standard deviation were also presented. These equations are summarized here:

Fundamental torsion period:
Mean:
(a) $T_{1 t}=0.015 \mathrm{~h}$
(b) $T_{1 t}=0.78 T_{1}$
(6.4a, b)
Mean - 1б: (a) $T_{1 t}=0.011 \mathrm{~h}$
(b) $T_{1 t}=0.60 T_{1}$
(6.5a, b)
Mean $+1 \sigma:$ (a) $T_{1 t}=0.018 \mathrm{~h}$
(b) $T_{1 t}=1.01 T_{1}$
$(6.6 a, b)$ 
Period of second translation modes:
Mean:
(a) $T_{2}=0.009 h^{0.9}$
(b) $T_{2}=0.31 T_{1}$
$(6.7 a, b)$
Mean $-1 \sigma:$ (a) $T_{2}=0.007 h^{0.9}$
(b) $T_{2}=0.28 T_{1}$
(6.8a, b)
Mean $+1 \sigma:$ (a) $T_{2}=0.012 h^{0.9}$
(b) $T_{2}=0.36 T_{1}$
(6.9a, b)

Period of second torsion mode:
Mean:
(a) $T_{2 t}=0.006 \mathrm{~h}$
(b) $T_{2 t}=0.38 T_{1 t}$
(6.10a, b)

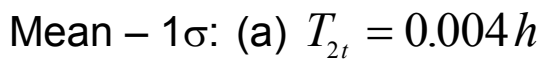
(b) $T_{2 t}=0.29 T_{1 t}$
$(6.11 a, b)$
Mean + 1б: (a) $T_{2 t}=0.009 \mathrm{~h}$
(b) $T_{2 t}=0.51 T_{1 t}$
$(6.12 a, b)$

In the above equations, height is in metres and the periods are in seconds.

These equations agree remarkably well with those proposed by other researchers for similar buildings. The equations based on the natural period of another vibration mode $\left(T_{l}\right.$ or $\left.T_{l t}\right)$ generally fit the measured data better than those based on building height. However, in a situation where one may be interested in estimating the natural period of one of these vibration modes $\left(T_{1}, T_{2}, T_{2 t}\right)$, it seems unlikely that the natural period of the required vibration mode $\left(T_{1}\right.$ or $\left.T_{1 t}\right)$ would be known a priori. Further, for the fundamental torsion period of a building, there is the additional problem of selecting which fundamental period to use (i.e. corresponding to which lateral direction) since the building will typically have different fundamental periods in its two lateral directions. On the other hand, building height is a more precise parameter that is likely known at the very early stages of the design process. Thus, in most situations, the equations based on building height would seem more appropriate to predict the natural periods of these vibration modes. 
A word of caution regarding the natural periods of the torsion modes however: most of the buildings considered in this study had shear walls concentrated in their central core. Buildings in which shear walls are distributed further apart in plan may prove to be stiffer than those studied here and it may not be wise to use the above equations to predict the torsion periods of such buildings. This requires further study.

Since the NBCC 2005 specifies that dynamic analysis methods should generally be used for the seismic design of buildings, and since such methods involve the combination of responses in several vibration modes, the code could eventually suggest methods to estimate the natural periods of higher vibration modes, in addition to the fundamental period. The models presented above could be a good starting point for the first six vibration modes of RCSW buildings. Note that for response spectrum analyses, it may be prudent to generally underestimate the natural period of the second translation modes. Hence, the mean minus one standard deviation equation would likely be appropriate for code purposes.

\subsection{Statement of originality}

The following is a summary of the original contributions of this research project:

- A database of the dynamic properties (natural periods, mode shapes, and damping) of 39 buildings in Montréal, 27 of which were reinforced concrete shear wall (RCSW) buildings, was compiled. To the author's knowledge, this is the largest database for these types of buildings in Canada. The results for all 39 buildings are published in a separate report and are available for further studies.

- The equation proposed in the 2005 National Building Code of Canada to estimate the fundamental periods of RCSW buildings 
was evaluated and an improved equation based on the results of regression analyses was developed.

- An inconsistency in the formula proposed in several building codes as an alternative method to estimate the fundamental periods of RCSW buildings was highlighted and a simple method to circumvent this inconsistency was suggested.

- The recommendations in the NBCC 2005 concerning damping, as well as other prediction models suggested in the literature, were evaluated and damping values to use for wind and seismic design of RCSW buildings were suggested.

- Simple models to predict the natural periods of the second translation modes of RCSW buildings, based on building height and the fundamental translation period, were proposed and these agreed with models proposed in the literature.

- Simple models to predict the natural periods of the fundamental torsion mode of RCSW buildings were also developed and these agreed with models proposed in the literature. These models may only be applicable to buildings having shear walls concentrated in their central core.

- Simple models to predict the period of the second torsion mode of RCSW buildings, based on building height and the fundamental torsion period, were proposed. To the author's knowledge, no other study addressed the issue of predicting the second torsion period. Again, these models may only be applicable to buildings with shear walls concentrated in their core.

- Finally, the uncertainty associated with each of these prediction models was quantified by providing equations corresponding to the mean, mean minus one standard deviation, and mean plus one standard deviation. 


\subsection{Limitations and recommendations for future work}

The above conclusions were based primarily on the measured dynamic properties of 27 RCSW buildings on the island of Montréal, identified from ambient vibration records. However, the natural periods and damping of buildings tend to be larger during earthquake ground shaking than during low-amplitude ambient motions. Therefore, the conclusions of this study only strictly apply to the low-amplitude behaviour of RCSW buildings. However, to the author's knowledge, no widely accepted method exists to quantify the changes in these parameters that can be expected from ambient to strong motions. If such methods were developed, the conclusions of this study could be revised to reflect the dynamic properties that can be expected during design-level vibrations.

Further, the dynamic properties of similar types of buildings, built in different regions, may vary due to differences in design and construction practices and seismicity. The results of this study should be representative of the behaviour of RCSW buildings in areas of moderate seismicity in Eastern Canada. However, similar studies should be performed in other urban centres across Canada to verify whether RCSW buildings constructed in different regions can be expected to have different dynamic properties.

Also, the dynamic properties of a building likely depend, to some extent, on the nature of the surrounding soil and its foundation details. However, the models considered in this study do not take account of this soilstructure interaction. If models can be developed to deal with the soilstructure interaction problem, this may improve predictions of the dynamic properties of buildings. In fact, several researchers have suggested that the observed increases in natural periods and damping in structures during earthquake shaking may be the result of nonlinear soil behavior. 
Hence, addressing the soil-structure interaction problem may prove critical to modeling the amplitude effects described above.

In the author's opinion, these three effects (amplitude effects, regional variations, and soil-structure interaction) are partly responsible for the observed variability in the dynamic properties of buildings. However, the fact that the models do not accurately capture all the parameters that contribute to these dynamic properties also plays a key role. For instance, models that better capture the distribution of stiffness and mass within a building could eventually lead to improved predictions of the natural periods of buildings. Moreover, better damping models representative of the various mechanisms of energy dissipation would clearly be useful to help engineers predict building response. However, in addition to providing better estimates of the dynamic properties, such models also need to be simple enough to be widely used in practice, and that is a considerable challenge.

Finally, this study only considered the dynamic properties of RCSW buildings. Similar studies should be performed for buildings made of different materials and employing different types of lateral load-resisting systems. The combined results could then form the basis of coherent recommendations in building codes. 


\section{Appendix A:}

\section{Estimation of damping from logarithmic decrement of autocorrelation function}




\section{Theory}

The autocorrelation function corresponding to the velocity response of a SDOF oscillator subjected to white noise excitation is (Clough and Penzien, 2003)

$$
R_{v v}(\tau)=\frac{\pi \omega_{n} S_{0}}{2 k^{2} \xi}\left(\omega_{n}{ }^{2} \cos \omega_{d} \tau-\frac{\omega_{n}{ }^{2} \xi}{\sqrt{1-\xi^{2}}} \sin \omega_{d} \tau\right) \exp \left(-\xi \omega_{n} \tau\right)
$$

where $S_{0}$ represents the constant power spectral density of the white noise excitation, $k$ is the stiffness of the oscillator, $\omega_{n}$ is its undamped natural frequency, $\omega_{d}$ is its damped natural frequency, $\xi$ is its viscous damping ratio, and $\tau$ represents time lag. It is clear from the above equation that the autocorrelation function decays exponentially in a manner similar to the free response of a SDOF oscillator with constant viscous damping. For this reason, the idea of using the logarithmic decrement to evaluate damping, which is commonly done using the free response, can be extended to the autocorrelation function of a SDOF oscillator under the assumption of white noise excitation.

Consider the autocorrelation function shown in Figure A-1. Suppose that a particular positive peak occurs at time $\tau_{l}$. It follows that the next positive peak will occur at time $\tau_{1}+T_{d}$. In other words, adjacent peaks are separated by a time equal to the damped natural period.

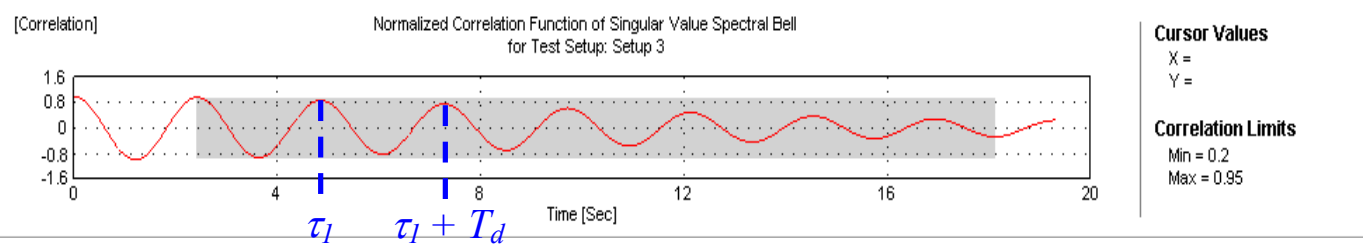

Figure A-1: Example autocorrelation function 
By comparing the values of the autocorrelation function at two adjacent peaks, separated by $T_{d}$, we notice that the constant terms in equation A-1 are identical, and that the cosine and sine terms return to their original values over the cycle of duration $T_{d}$. Thus, by taking the ratio of the autocorrelation values at two adjacent peaks, the expression simplifies significantly:

$$
\frac{R_{v v}\left(\tau_{1}\right)}{R_{v v}\left(\tau_{1}+T_{d}\right)}=\frac{\exp \left(-\xi \omega_{n} \tau_{1}\right)^{-\xi \omega \tau_{1}}}{\exp \left(-\xi \omega_{n}\left(\tau_{1}+T_{d}\right)\right)}=\exp \left(\xi \omega_{n} T_{d}\right)
$$

We define the logarithmic decrement $\delta$ as the natural logarithm of the ratio of the autocorrelation function at two adjacent peaks:

$$
\delta=\ln \left(\frac{R_{v v}\left(\tau_{1}\right)}{R_{v v}\left(\tau_{1}+T_{d}\right)}\right)
$$

It follows that

$$
\delta=\xi \omega_{n} T_{d}
$$

Considering now the relation between the undamped natural frequency and the damped natural period,

$$
\omega_{n}=\frac{2 \pi}{T_{d} \sqrt{1-\xi^{2}}}
$$

we obtain

$$
\delta=\frac{2 \pi \xi}{\sqrt{1-\xi^{2}}}
$$


Rearranging and isolating $\xi$, we get

$$
\xi=\frac{\delta}{\sqrt{4 \pi^{2}+\delta^{2}}}
$$

In this way, once an estimate of the logarithmic decrement is available, the damping ratio of the SDOF oscillator can be obtained from the above equation. This is exactly the same result as would be obtained considering the free response of an SDOF oscillator with constant viscous damping, or, under the assumption of light damping, using any other response indicator (displacement or acceleration).

\section{Implementation in ARTeMIS}

In ARTeMIS, using the enhanced frequency domain decomposition method (EFDD), damping is estimated as described above, but with one minor variation: the logarithmic decrement is calculated considering all peaks within a given range of time lags, rather than considering only two adjacent peaks.

The natural logarithms of the autocorrelation function at each peak are plotted against the time at which these peaks occur, yielding a plot similar to that of Figure A-2.

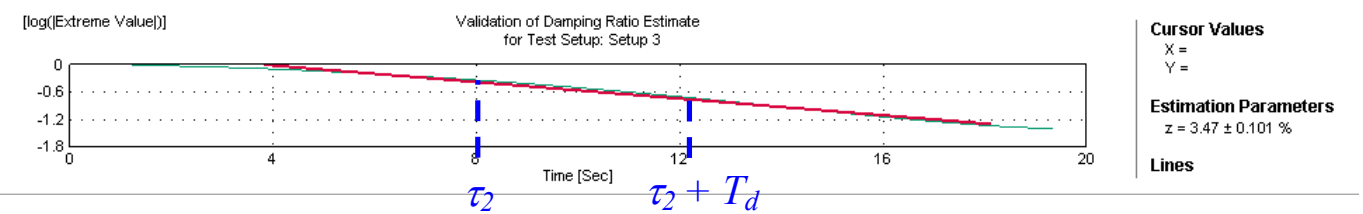

Figure A-2: Estimate of damping using logarithmic decrement 
Note that the user must select the decaying portion of the autocorrelation function that will be considered to calculate the logarithmic decrement. In practice, the beginning of the autocorrelation function is often corrupted by broadband noise and the uncertainty associated with the estimation of the autocorrelation function increases for large time lags (Structural Vibration Solutions A/S, 2009b). For these reasons, it is best to consider only a portion of the autocorrelation function, away from the beginning, where the decaying sinusoidal behaviour is evident. This is done by setting maximum and minimum limits on the value of the autocorrelation function, as shown by the shaded region in Figure A-1.

A linear regression is then performed considering only the selected region of the autocorrelation function (Brincker et al., 2001a). The slope of the regression line (indicated in red in Figure A-2) corresponds to the ratio of the logarithmic decrement to the damped natural period. Consider the logarithm of the autocorrelation function at an arbitrary time lag $\tau_{2}$ and at a time lag of $\tau_{2}+T_{d}$. The slope of the regression line can therefore be expressed as

$$
m=\frac{\ln \left(R_{v v}\left(\tau_{2}+T_{d}\right)\right)-\ln \left(R_{v v}\left(\tau_{2}\right)\right)}{\left(\tau_{2}+T_{d}\right)-\tau_{2}}=-\frac{\delta}{T_{d}}
$$

Therefore, the logarithmic decrement depends on the value of the damped natural period, which is estimated by counting the zero crossings in the autocorrelation function, as described in section 3.2.2.3.

To summarize, the slope $m$ in Figure A-2 is obtained by regression analysis, the natural period is estimated by considering the zero crossings of the autocorrelation function, and these two values are used to calculate the logarithmic decrement. 


$$
\delta=-m T_{d}
$$

The logarithmic decrement is then used to estimate the damping ratio using equation A-7. 


\section{Appendix B:}

Identification of the first two vibration modes of RCSW-1 
Ambient vibration tests were performed in building RCSW-1 on August 18, 2009. The modal parameters corresponding to six vibration modes were identified and are summarized in Gilles (2010). To illustrate the estimation of the modal parameters using enhanced frequency domain decomposition (EFDD), the detailed identification of the first two (lowest frequency) vibration modes is presented here.

RCSW-1 is a 15-storey reinforced concrete building, with shear walls in the central core. Figure B-1 shows a plan view of the building. Velocity time histories were recorded at three locations (as indicated in Figure B-1) on each of floors $2,5,8,11$, and 15 , as well as at the second basement level, for a total of 18 measurement locations.

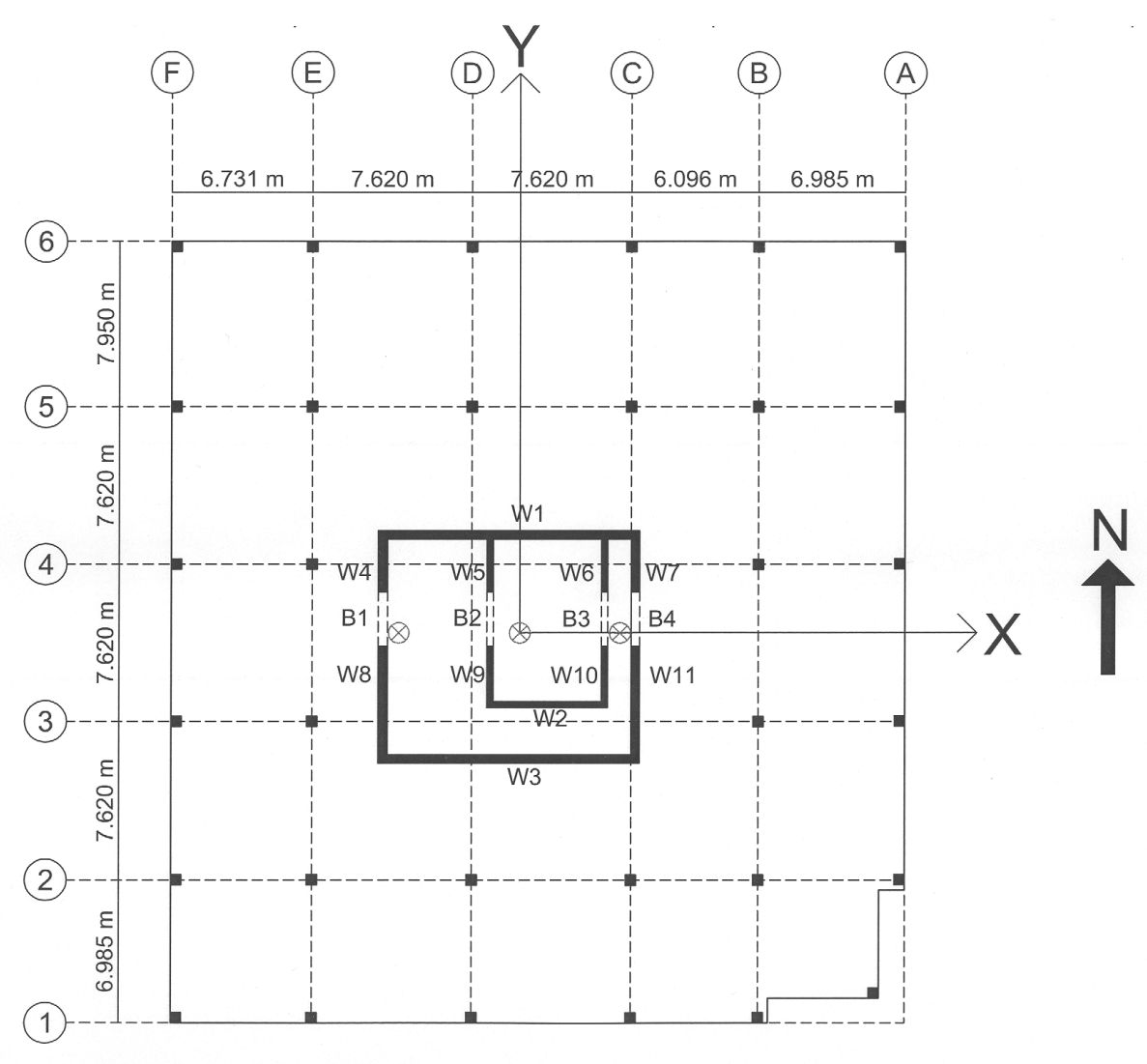

Q Measurement points

Figure B-1: Plan view of RCSW-1 
The reference sensor was placed on the West side of floor 15. The first record was taken with both sensors side by side at this location, and the roving sensor was moved to a different location for each subsequent setup, as shown in Table B-1. The first three records, as well as a dummy record taken immediately after the last measurement, were started using the remote control starter (RCS). Inspection of the time stamps in these data records suggested that the internal clock of the reference data acquisition system (DAS) was $1.000 \mathrm{~s}$ ahead of that of the roving DAS. This difference was corrected prior to synchronizing the data. Otherwise, the data for all measurement setups in which the RCS was not used (setups 4 to 18) were synchronized as explained in section 3.2.1.2.

Table B-1: Sensor locations for different measurement setups

\begin{tabular}{|c|c|c|}
\hline Measurement setup & Reference location & Roving location \\
\hline 1 & 15-West & 15-West \\
\hline 2 & 15-West & 15-Centre \\
\hline 3 & 15-West & 15-East \\
\hline 4 & 15-West & 11-West \\
\hline 5 & 15-West & 11-Centre \\
\hline 6 & 15-West & 11-East \\
\hline 7 & 15-West & 8-West \\
\hline 8 & 15-West & 8-Centre \\
\hline 9 & 15-West & 8-East \\
\hline 10 & 15-West & 5-West \\
\hline 11 & 15-West & 5-Centre \\
\hline 12 & 15-West & 5-East \\
\hline 13 & 15-West & 2-West \\
\hline 14 & 15-West & 2-Centre \\
\hline 15 & 15-West & 2-East \\
\hline 16 & 15-West & B2-West \\
\hline 17 & 15-West & B2-Centre \\
\hline 18 & 15-West & B2-East \\
\hline
\end{tabular}

The synchronized data were then input into ARTeMIS for analysis. The maximum frequency of interest was limited to $25 \mathrm{~Hz}$, and 1024 frequency lines in the range from 0 to $25 \mathrm{~Hz}$ were selected for the estimation of spectral densities, leading to a frequency spacing of $0.2441 \mathrm{~Hz}$ in the singular value plot. Figure B-2 shows the average normalized singular value plot thus obtained. 


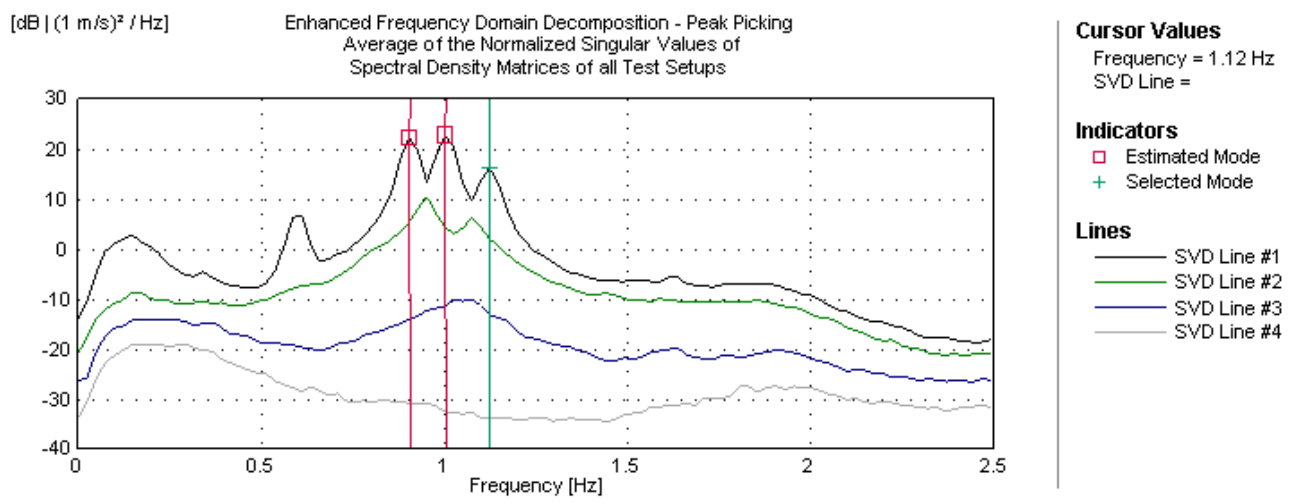

Figure B-2: Singular value plot for RCSW-1

Notice that a wide peak is visible at $0.2 \mathrm{~Hz}$. Recall that the transfer function of the sensor used in this study is flat above a frequency of 0.2 $\mathrm{Hz}$. However, the transfer function ramps up from 0 to a constant value over the range of 0 to $0.2 \mathrm{~Hz}$, which explains the peak in the singular value plot. This peak therefore does not represent a resonant frequency of the building.

Notice also a second peak at around $0.6 \mathrm{~Hz}$. This was considered as a potential resonant frequency; however, inspection of the corresponding mode shape (using FDD and not EFDD) showed that it was identical to that of the adjacent peak near $0.9 \mathrm{~Hz}$. Since the singular values are in logarithmic scale (see equation 3.6), the peak near $0.9 \mathrm{~Hz}$ has more than 10 times the energy of the peak near $0.6 \mathrm{~Hz}$. For this reason, the peak at $0.9 \mathrm{~Hz}$ was selected as the first resonant frequency. The adjacent peak at 1.0 Hz was selected as the second resonant frequency.

\section{Identification of first two vibration modes using EFDD}

Once the candidate resonant frequencies were selected from the average normalized singular value plot, the frequency, mode shape, and damping 
ratio for each mode were then obtained using EFDD, as described in section 3.2.2.3.

For each mode and for each setup, the SDOF bell was identified from the singular value plot for that setup. A different MAC rejection criterion, $\Omega$, was selected for each setup to capture as much of the resonance peak as possible. The program then automatically padded the function with zeros for all remaining frequencies, and the SDOF bell was transformed to the frequency domain using an inverse Fourier transform, yielding an SDOF autocorrelation function, which was normalized to a maximum value of 1 . Further, two additional graphs - one showing the logarithms of the extreme (peak) values of the autocorrelation function plotted against time, and the other showing the zero crossings against time - were automatically generated. The decaying portion of the autocorrelation function was then captured by selecting the maximum and minimum correlation limits. Typical values were respectively 0.95 and 0.2 , but different values were selected in certain setups. Regression lines were then automatically calculated in the extreme value and zero crossings plots considering only the selected portion of the autocorrelation function. These plots were examined to ensure that they were approximately linear and that they coincided reasonably well with the regression lines, in the selected region. From these regression lines, improved estimates of frequency and damping were calculated, as described in section 3.2.2.3.

Finally, the values of frequency and damping from each setup were averaged to obtain overall estimates of these quantities and their associated uncertainty, as shown in Table B-2. The mode shapes were obtained from the singular vectors in each setup, as described in section 3.2.2.3. These are shown in Figure B-3. The following pages show the identification of the SDOF bell and the validation of the damping ratio and 
frequency estimates for each setup, for each of the first two vibration modes.

Table B-2: Modal parameters of first two vibration modes identified in each setup

\begin{tabular}{|c|c|c|c|c|c|c|c|c|c|c|}
\hline \multirow{2}{*}{ Setup } & \multicolumn{5}{|c|}{ Mode 1} & \multicolumn{5}{|c|}{ Mode 2} \\
\hline & $\Omega$ & $C_{\min }$ & $C_{\max }$ & $f(\mathrm{~Hz})$ & $\xi(\%)$ & $\Omega$ & $C_{\min }$ & $C_{\max }$ & $f(\mathrm{~Hz})$ & $\xi(\%)$ \\
\hline 1 & 0.578 & 0.20 & 0.95 & 0.910 & 2.357 & 0.670 & 0.20 & 0.95 & 1.003 & 1.962 \\
\hline 2 & 0.930 & 0.20 & 0.95 & 0.906 & 1.853 & 0.700 & 0.20 & 0.95 & 0.994 & 2.164 \\
\hline 3 & 0.700 & 0.20 & 0.95 & 0.907 & 2.249 & 0.700 & 0.20 & 0.95 & 1.005 & 2.012 \\
\hline 4 & 0.910 & 0.20 & 0.95 & 0.908 & 2.356 & 0.800 & 0.20 & 0.95 & 1.009 & 1.678 \\
\hline 5 & 0.800 & 0.24 & 0.95 & 0.903 & 1.778 & 0.818 & 0.20 & 0.95 & 1.006 & 1.972 \\
\hline 6 & 0.700 & 0.20 & 0.95 & 0.911 & 2.572 & 0.730 & 0.20 & 0.95 & 1.007 & 1.948 \\
\hline 7 & 0.717 & 0.20 & 0.95 & 0.909 & 2.055 & 0.620 & 0.20 & 0.95 & 1.002 & 1.803 \\
\hline 8 & 0.930 & 0.20 & 0.95 & 0.909 & 2.278 & 0.683 & 0.20 & 0.95 & 1.005 & 1.932 \\
\hline 9 & 0.600 & 0.20 & 0.95 & 0.903 & 2.019 & 0.600 & 0.20 & 0.95 & 1.003 & 1.511 \\
\hline 10 & 0.910 & 0.20 & 0.95 & 0.903 & 2.060 & 0.550 & 0.20 & 0.95 & 1.003 & 2.879 \\
\hline 11 & 0.782 & 0.20 & 0.95 & 0.907 & 2.037 & 0.630 & 0.20 & 0.95 & 1.001 & 1.758 \\
\hline 12 & 0.560 & 0.20 & 0.95 & 0.908 & 1.851 & 0.700 & 0.20 & 0.95 & 1.003 & 1.787 \\
\hline 13 & 0.700 & 0.20 & 0.95 & 0.911 & 2.208 & 0.790 & 0.20 & 0.95 & 1.010 & 2.461 \\
\hline 14 & 0.612 & 0.20 & 0.95 & 0.911 & 2.188 & 0.555 & 0.20 & 0.95 & 1.008 & 2.092 \\
\hline 15 & 0.750 & 0.20 & 0.95 & 0.905 & 1.788 & 0.671 & 0.20 & 0.95 & 1.001 & 2.339 \\
\hline 16 & 0.700 & 0.20 & 0.95 & 0.905 & 2.124 & 0.650 & 0.20 & 0.95 & 1.010 & 1.821 \\
\hline 17 & 0.550 & 0.20 & 0.95 & 0.903 & 2.088 & 0.590 & 0.20 & 0.95 & 1.003 & 1.909 \\
\hline 18 & 0.800 & 0.20 & 0.95 & 0.906 & 2.249 & 0.620 & 0.20 & 0.95 & 1.010 & 2.067 \\
\hline \multicolumn{4}{|c|}{ Mean } & 0.907 & 2.117 & \multicolumn{3}{|c|}{ Mean } & 1.005 & 2.005 \\
\hline \multicolumn{4}{|c|}{ Stand Dev } & 0.003 & 0.215 & \multicolumn{3}{|c|}{ Stand Dev } & 0.004 & 0.314 \\
\hline $\begin{array}{l}\Omega \\
C_{\text {min }}: \\
C_{\text {max }}: \\
f: \\
\xi\end{array}$ & $\begin{array}{l}\text { MAC } \\
\text { Minim } \\
\text { Maxin } \\
\text { Frequ } \\
\text { Damp }\end{array}$ & $\begin{array}{l}\text { jectio } \\
\text { m cor } \\
\text { Im co } \\
\text { acy (I } \\
\text { ig rati }\end{array}$ & $\begin{array}{l}\text { criter } \\
\text { lation } \\
\text { elation } \\
)^{2} \% \mathrm{cr}\end{array}$ & $\begin{array}{l}\text { mit } \\
\text { imit } \\
\text { cal) }\end{array}$ & & & & & & \\
\hline
\end{tabular}

\begin{tabular}{|c|c|c|c|}
\hline 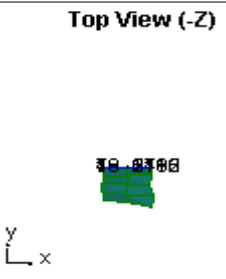 & $\begin{array}{l}\text { Side view }(+\mathrm{X}) \\
\qquad\left.\right|_{18} ^{39} \\
19 \\
18\end{array}$ & $\begin{array}{l}\text { Top View }(-Z) \\
\text { - }\end{array}$ & 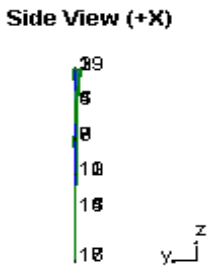 \\
\hline 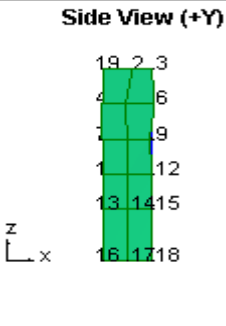 & $\begin{array}{l}\text { 3D view } \\
x_{1}^{z} \times\end{array}$ & 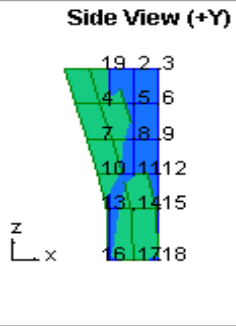 & 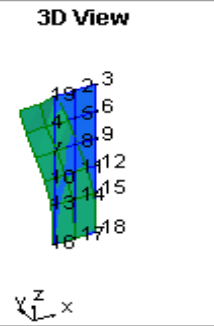 \\
\hline
\end{tabular}

(a)

(b)

Figure B-3: Mode shapes corresponding to (a) Mode 1; (b) Mode 2 


\section{Identification of Mode 1:}

\section{Setup 1:}
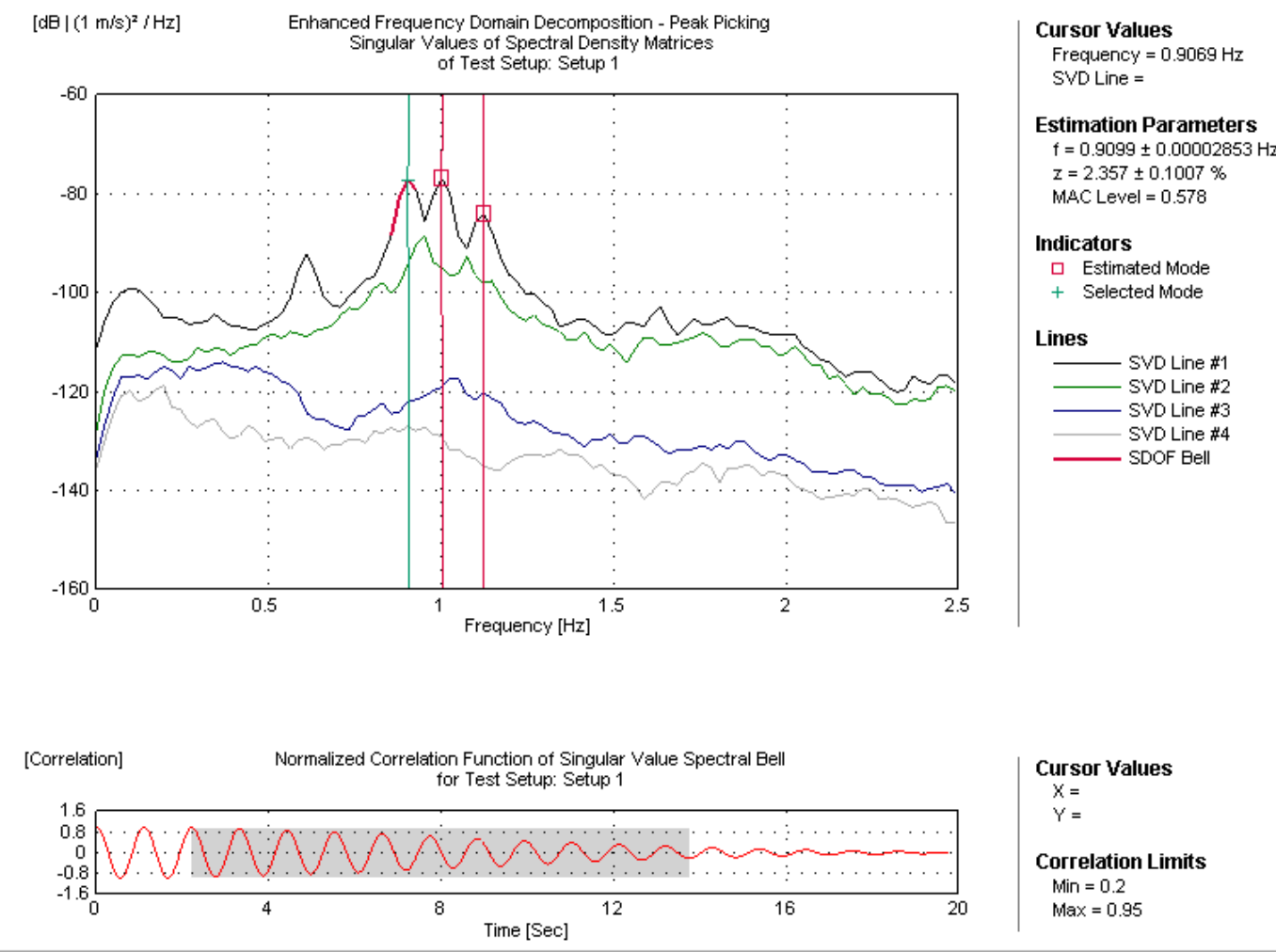
Cursor Values
$X=$
$Y=$
Correlation Limits
Min $=0.2$
$\operatorname{Max}=0.95$

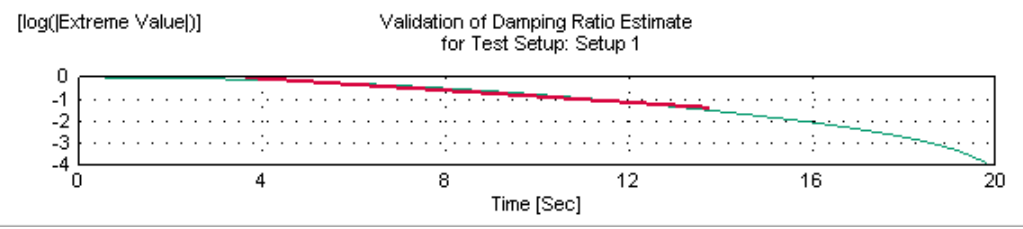

Cursor Values

$X=$
$Y=$

Estimation Parameters

$z=2.357 \pm 0.1007 \%$

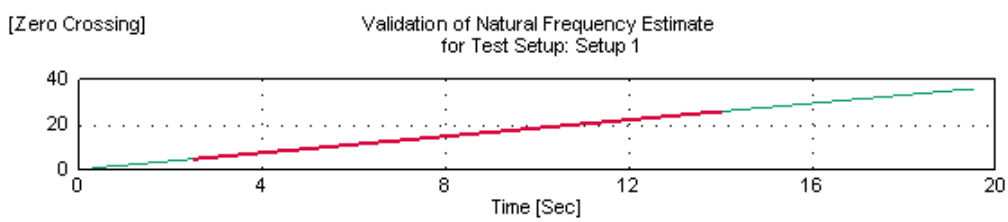

Cursor Values

$X=$

Estimation Parameters $f=0.91 \pm 0.0000285 \mathrm{~Hz}$ 


\section{Setup 2:}

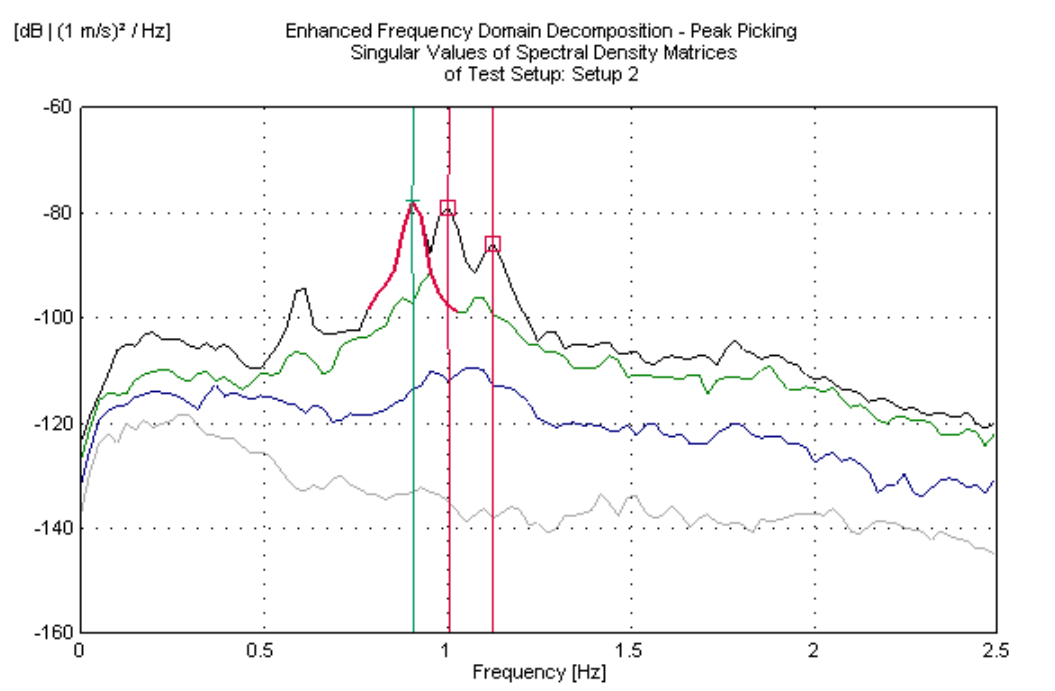

Cursor Values

Frequency $=0.9069 \mathrm{~Hz}$ SVD Line $=$

Estimation Parameters $\mathrm{f}=0.9064 \pm 0.000009311 \mathrm{H}_{2}$ $z=1.853 \pm 0.0476 \%$ MAC Level $=0.93$

Indicators

$\square$ Estimated Mode + Selected Mode

Lines

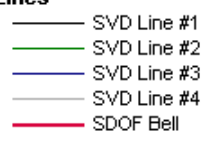

[Correlation] Normalized Correlation Function of Singular Value Spectral Bell for Test Setup: Setup 2

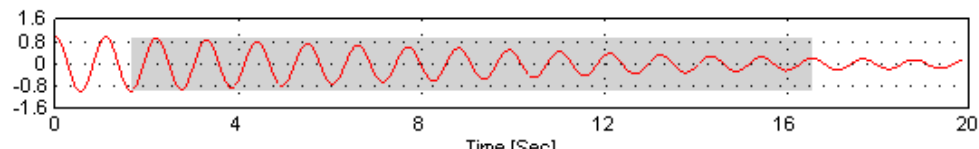

Cursor Values

$X=$

Correlation Limits

Min $=0.2$

$\operatorname{Max}=0.95$

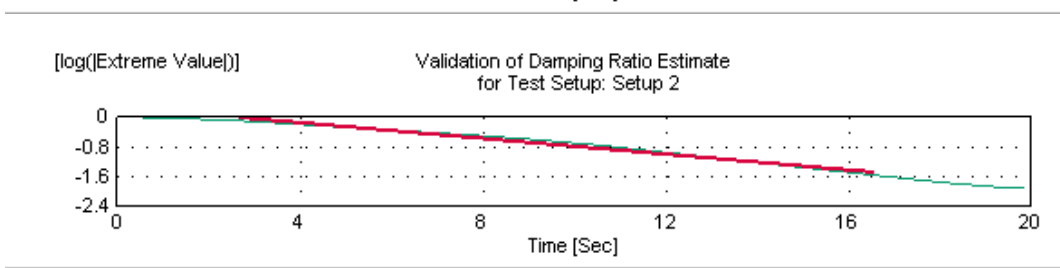

Cursor Values

$X=$
$Y=$

Estimation Parameters $z=1.853 \pm 0.0476 \%$

[Zero Crossing] Validation of Natural Frequency Estimate

for Test Setup: Setup 2

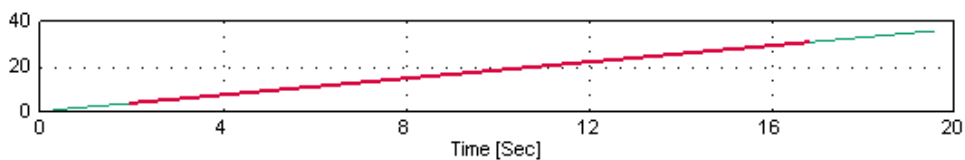

Estimation Parameters $f=0.906 \pm 0.00000931 \mathrm{~Hz}$ 


\section{Setup 3:}

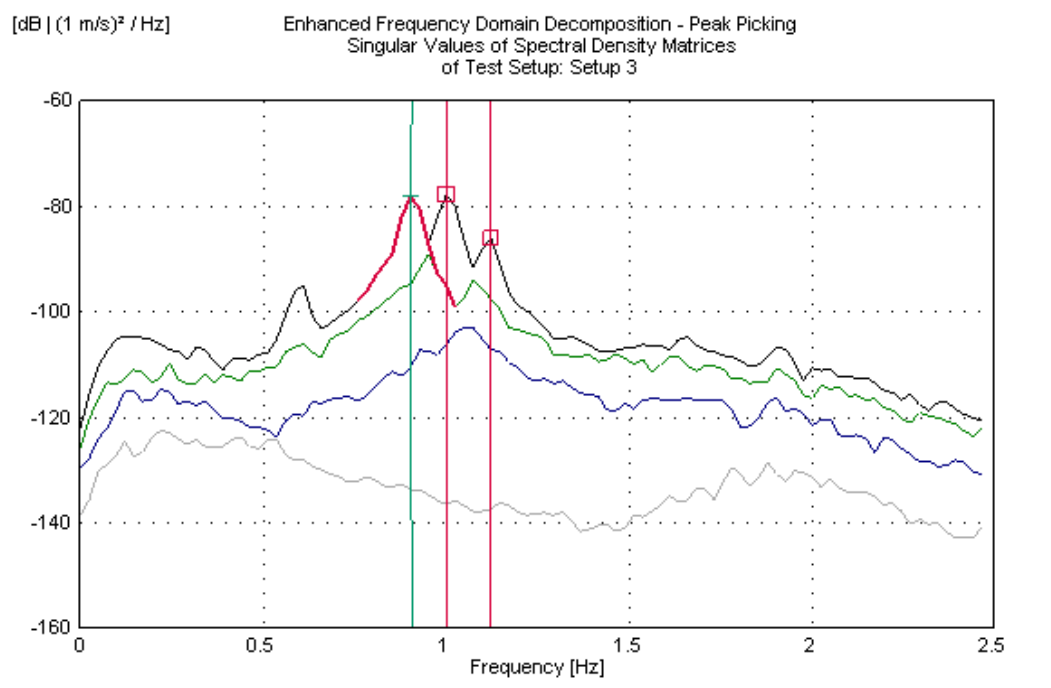

Cursor Values

Frequency $=0.9069 \mathrm{~Hz}$ SVD Line =

Estimation Parameters $f=0.9067 \pm 0.00001236 \mathrm{~Hz}$

$z=2.249 \pm 0.05311 \%$

MAC Level $=0.7$

Indicators

$\square$ Estimated Mode

+ Selected Mode

Lines

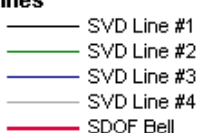

[Correlation] Normalized Correlation Function of Singular Value Spectral Bell for Test Setup: Setup 3

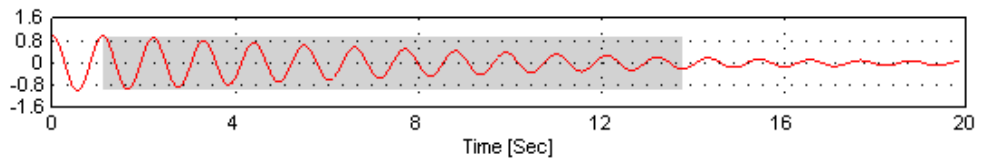

Cursor Values

$X=$

Correlation Limits

$\operatorname{Min}=0.2$

$\operatorname{Max}=0.95$

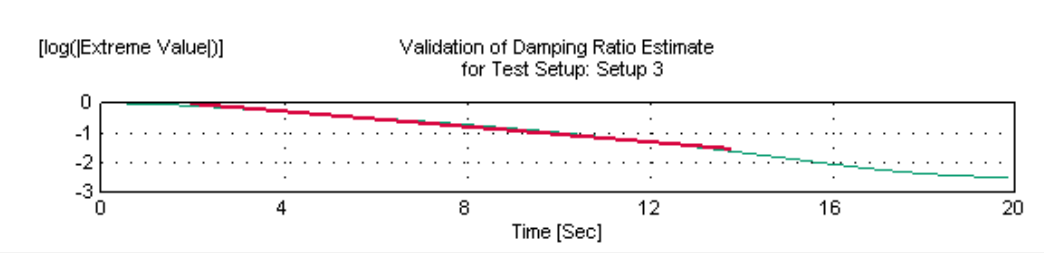

Cursor Values

$X=$

Estimation Parameters

$z=2.249 \pm 0.05311 \%$

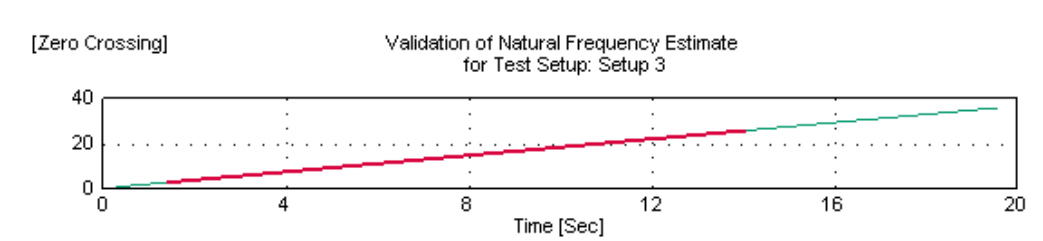

Cursor Values

$X=$
$Y=$

Estimation Parameters $t=0.907 \pm 0.0000124 \mathrm{~Hz}$ 


\section{Setup 4:}

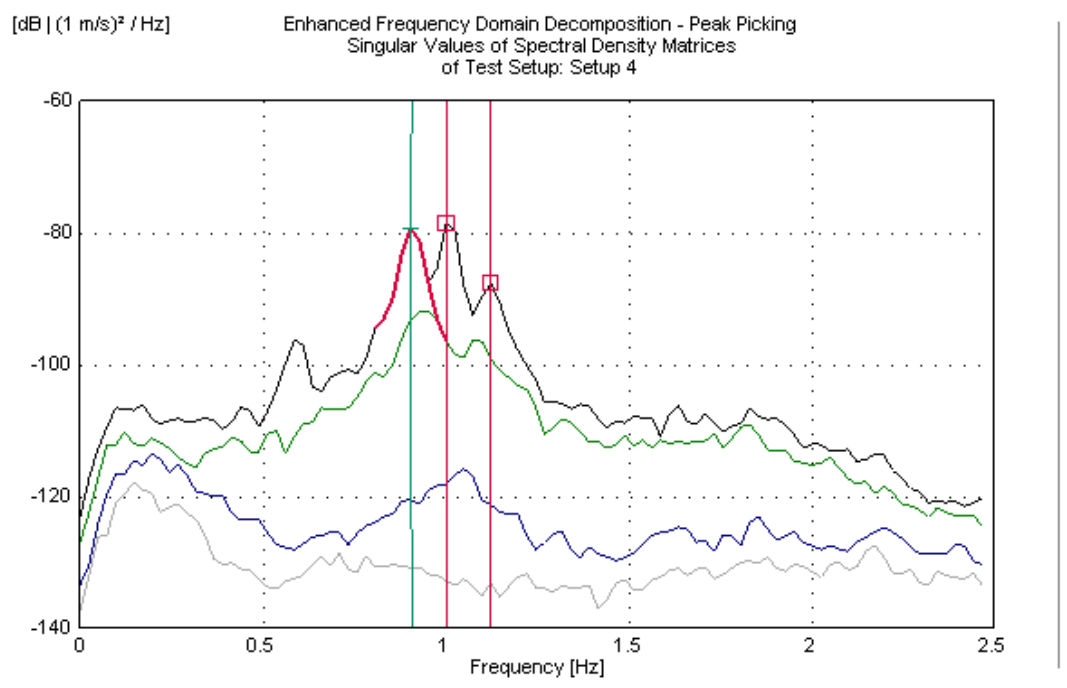

Cursor Values

Frequency $=0.9069 \mathrm{~Hz}$ SVD Line =

Estimation Parameters $t=0.9081 \pm 0.00001356 \mathrm{~Hz}$

$z=2.356 \pm 0.05446 \%$

MAC Level $=0.91$

Indicators

$\square$ Estimated Mode

+ Selected Mode

Lines

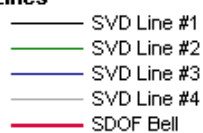

[Correlation] Normalized Correlation Function of Singular Value Spectral Bell for Test Setup: Setup 4

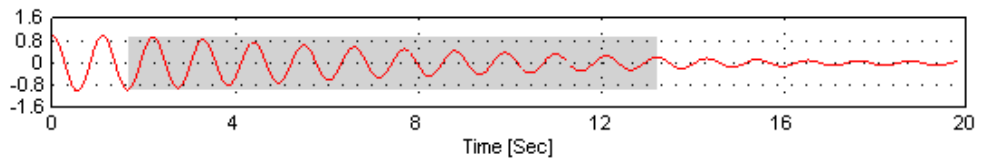

Cursor Values

$X=$

Correlation Limits

$\operatorname{Min}=0.2$

$\operatorname{Max}=0.95$

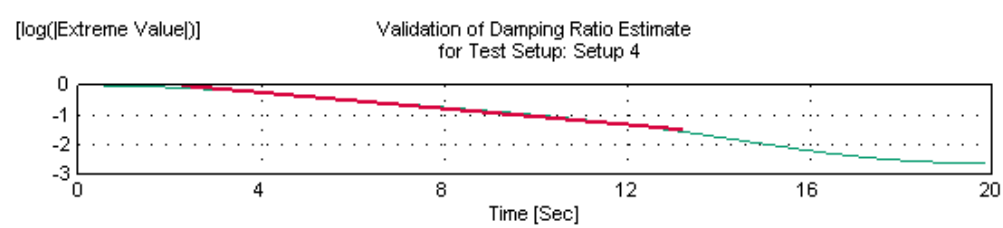

Cursor Values

$X=$

Estimation Parameters

$z=2.356 \pm 0.05446 \%$

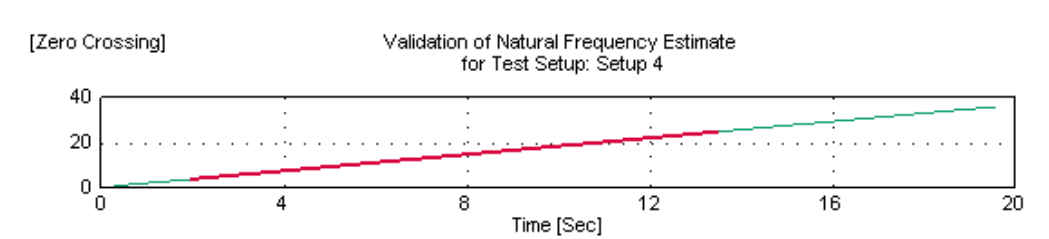

Cursor Values

$X=$
$Y=$

Estimation Parameters $t=0.908 \pm 0.0000136 \mathrm{~Hz}$ 


\section{Setup 5:}

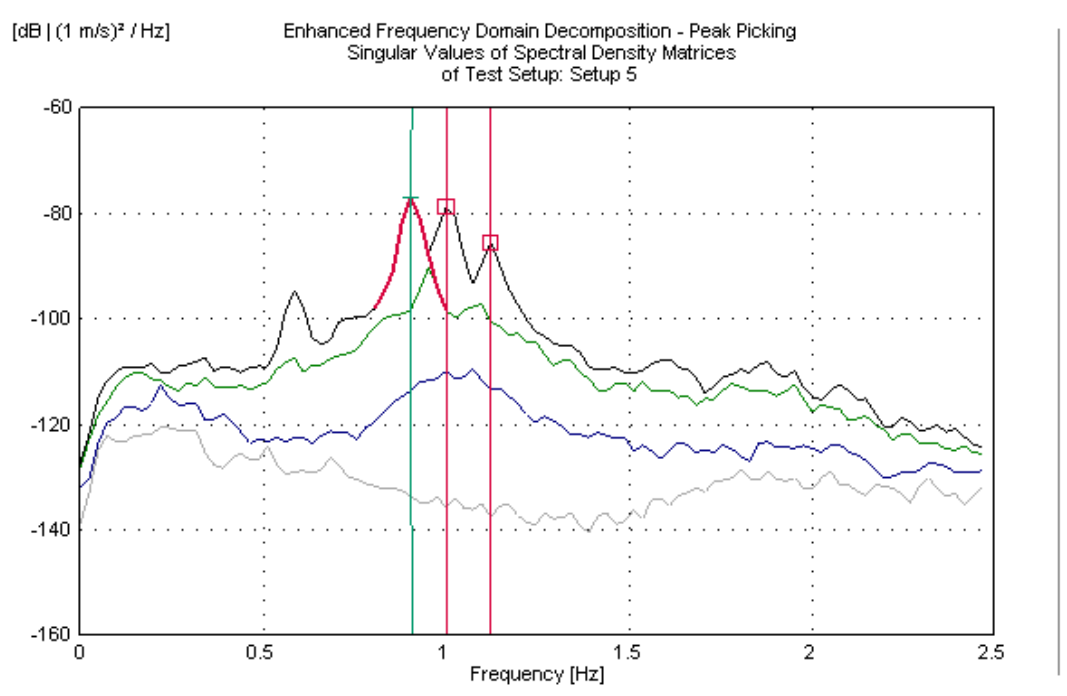

Cursor Values

Frequency $=0.9069 \mathrm{~Hz}$ SVD Line =

Estimation Parameters $f=0.9033 \pm 0.000007304 \mathrm{H}_{2}$

$z=1.778 \pm 0.02306 \%$

MAC Level $=0.8$

Indicators

$\square$ Estimated Mode

+ Selected Mode

Lines

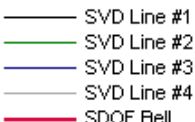

[Correlation] Normalized Correlation Function of Singular Value Spectral Bell for Test Setup: Setup 5

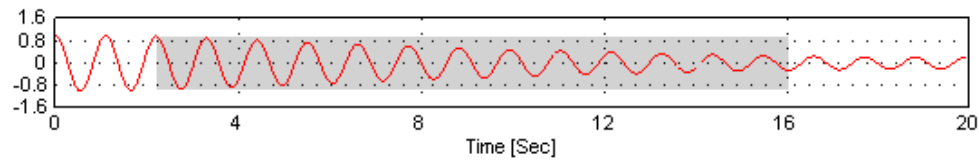

$[\log (\mid$ Extreme Value $)]$

Validation of Damping Ratio Estimate

for Test Setup: Setup 5

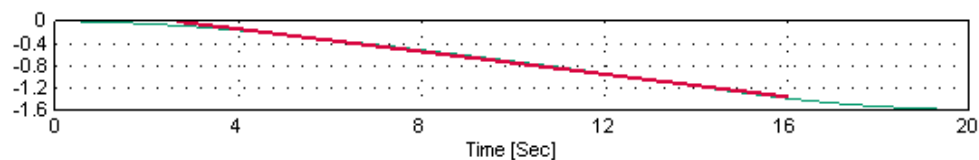

Cursor Values

$X=$

Correlation Limits

$\operatorname{Min}=0.24$

$\operatorname{Max}=0.95$

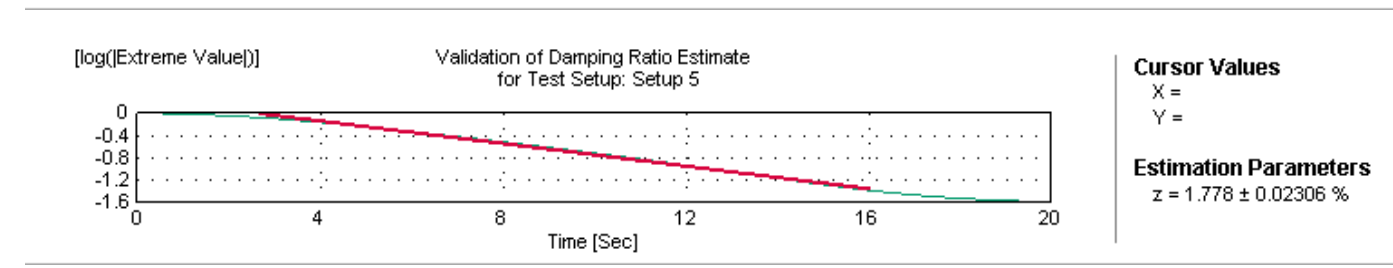

[Zero Crossing]

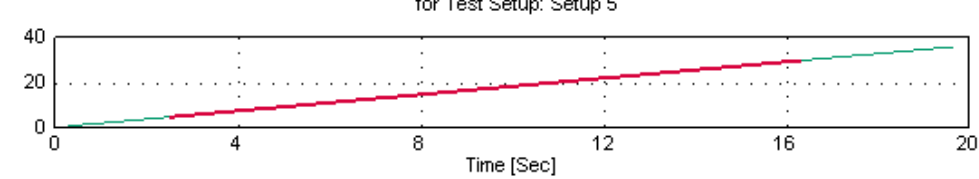

Cursor Values

$X=$
$Y=$

Estimation Parameters $t=0.903 \pm 0.0000073 \mathrm{~Hz}$ 


\section{Setup 6:}

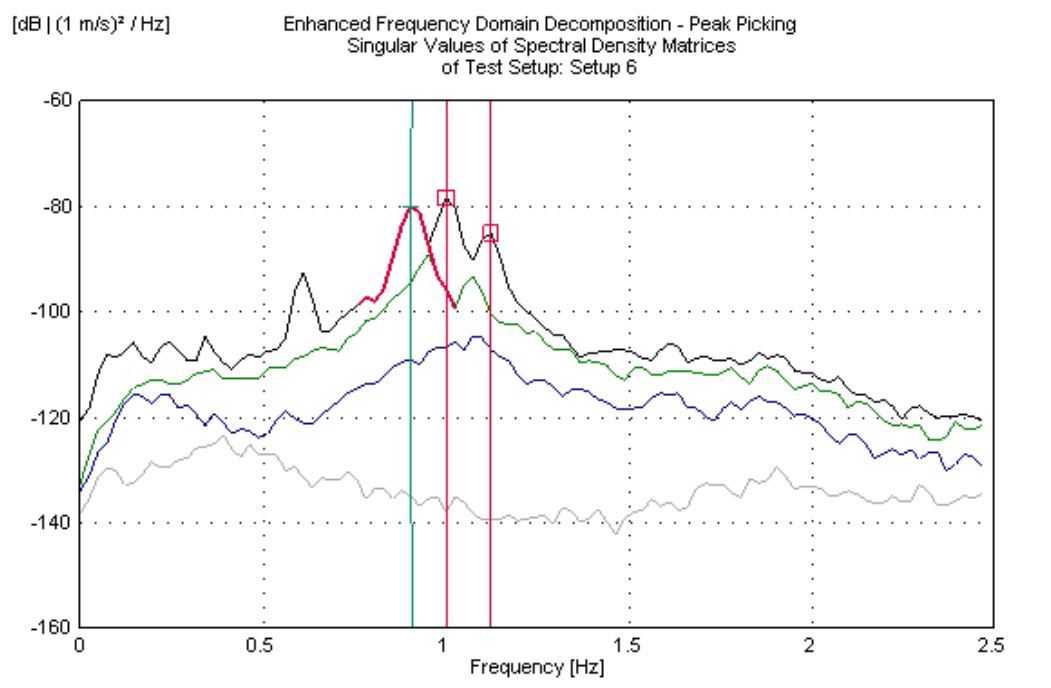

Cursor Values

Frequency $=0.9069 \mathrm{~Hz}$ SVD Line =

Estimation Parameters $f=0.911 \pm 0.00002224 \mathrm{~Hz}$

$z=2.572 \pm 0.08165 \%$

MAC Level $=0.7$

Indicators

$\square$ Estimated Mode

+ Selected Mode

Lines

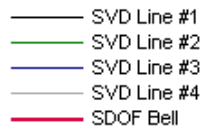

[Correlation] Normalized Correlation Function of Singular Value Spectral Bell for Test Setup: Setup 6

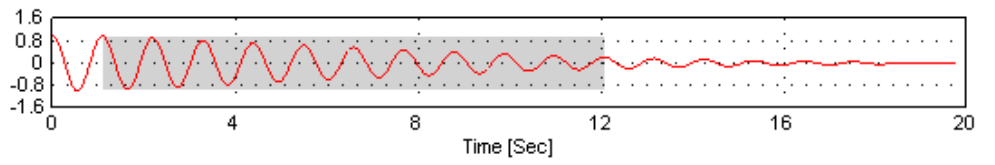

Cursor Values

$X=$

Correlation Limits

$\operatorname{Min}=0.2$

$\operatorname{Max}=0.95$

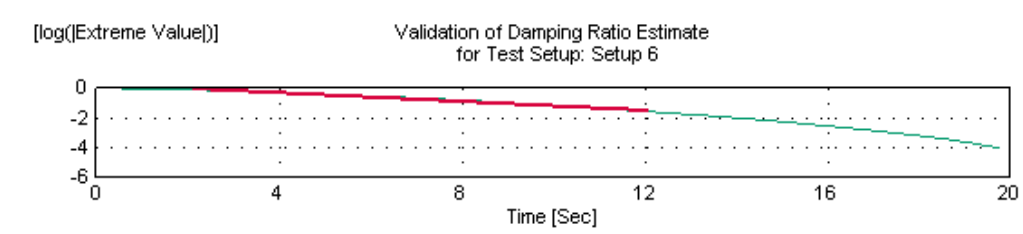

Cursor Values

$X=$

Estimation Parameters

$z=2.572 \pm 0.08165 \%$

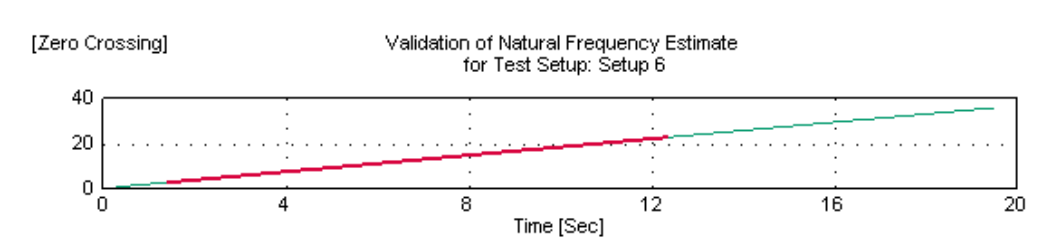

Cursor Values

$X=$
$Y=$

Estimation Parameters $t=0.911 \pm 0.0000222 \mathrm{~Hz}$ 


\section{Setup 7:}

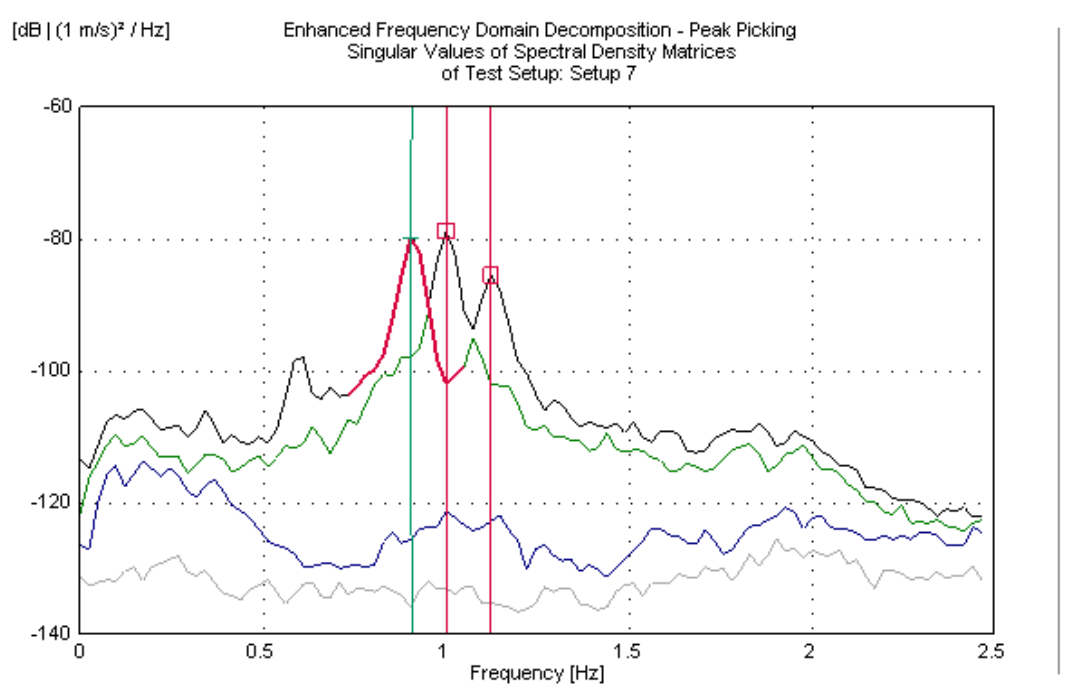

Cursor Values

Frequency $=0.9069 \mathrm{~Hz}$ SVD Line =

Estimation Parameters $f=0.9089 \pm 0.00001134 \mathrm{~Hz}$

$z=2.055 \pm 0.05253 \%$

MAC Level $=0.7166$

Indicators

$\square$ Estimated Mode

+ Selected Mode

Lines

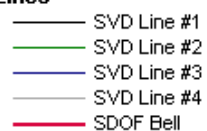

[Correlation] Normalized Correlation Function of Singular Value Spectral Bell for Test Setup: Setup 7

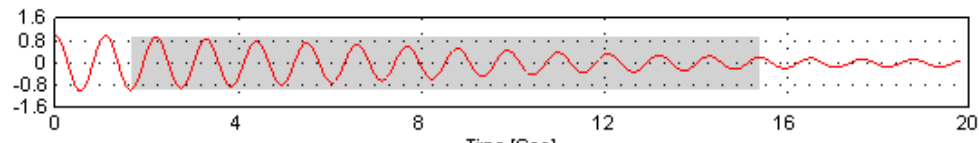

Time [Sec]

for Test Setup: Setup 7

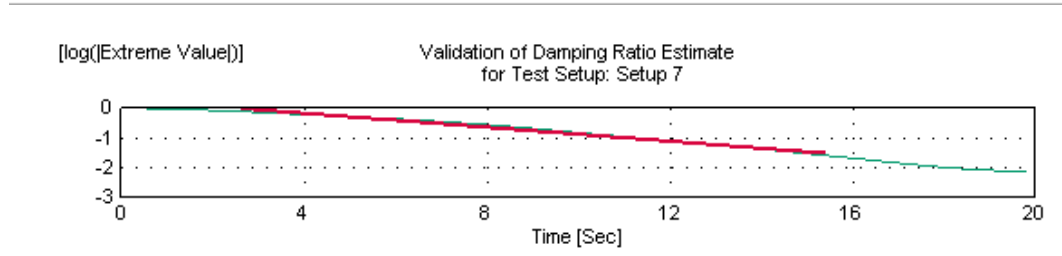

Cursor Values

$X=$

Correlation Limits

$\operatorname{Min}=0.2$

$\operatorname{Max}=0.95$

Cursor Values

$X=$

Estimation Parameters

$z=2.055 \pm 0.05253 \%$

[Zero Crossing] Validation of Natural Frequency Estimate

for Test Setup: Setup 7

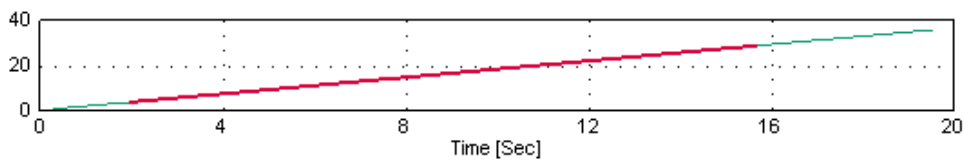

Cursor Values

$X=$
$Y=$

Estimation Parameters $f=0.909 \pm 0.0000113 \mathrm{~Hz}$ 


\section{Setup 8:}

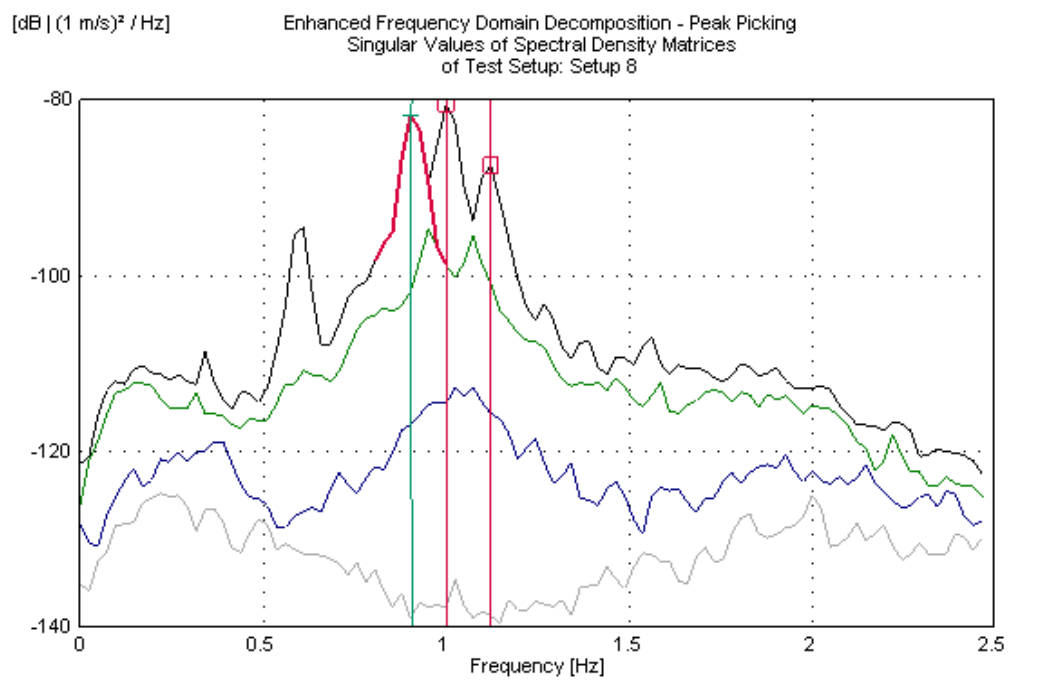

Cursor Values

Frequency $=0.9069 \mathrm{~Hz}$ SVD Line =

Estimation Parameters $f=0.9087 \pm 0.00001864 \mathrm{~Hz}$

$z=2.278 \pm 0.06976 \%$

MAC Level $=0.93$

Indicators

$\square$ Estimated Mode

+ Selected Mode

Lines

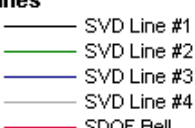

[Correlation] Normalized Correlation Function of Singular Value Spectral Bell for Test Setup: Setup 8

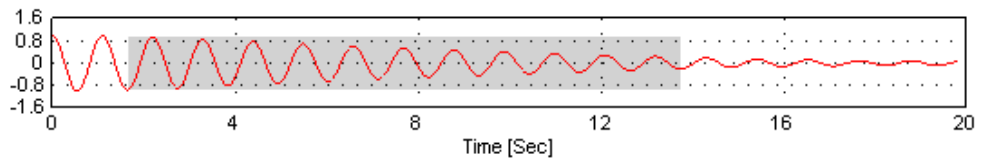

Cursor Values

$X=$

Correlation Limits

$\operatorname{Min}=0.2$

$\operatorname{Max}=0.95$

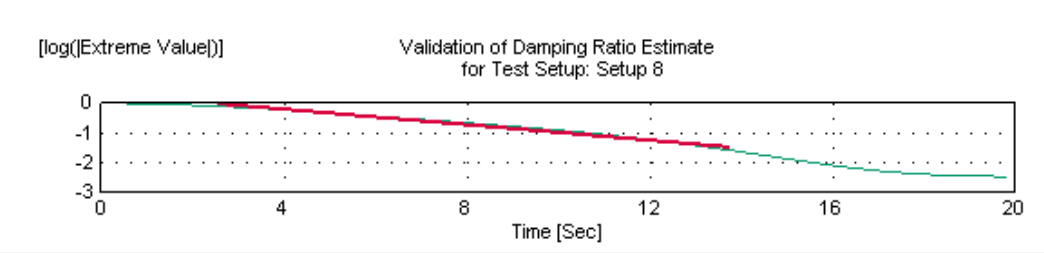

Cursor Values

$X=$

Estimation Parameters

$z=2.278 \pm 0.06976 \%$

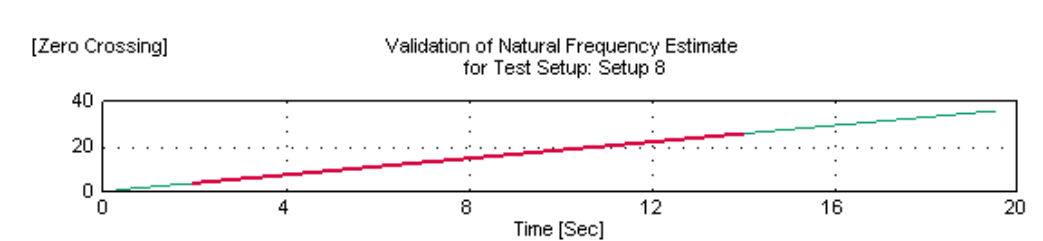

Cursor Values

$X=$
$Y=$

Estimation Parameters $t=0.909 \pm 0.0000186 \mathrm{~Hz}$ 


\section{Setup 9:}

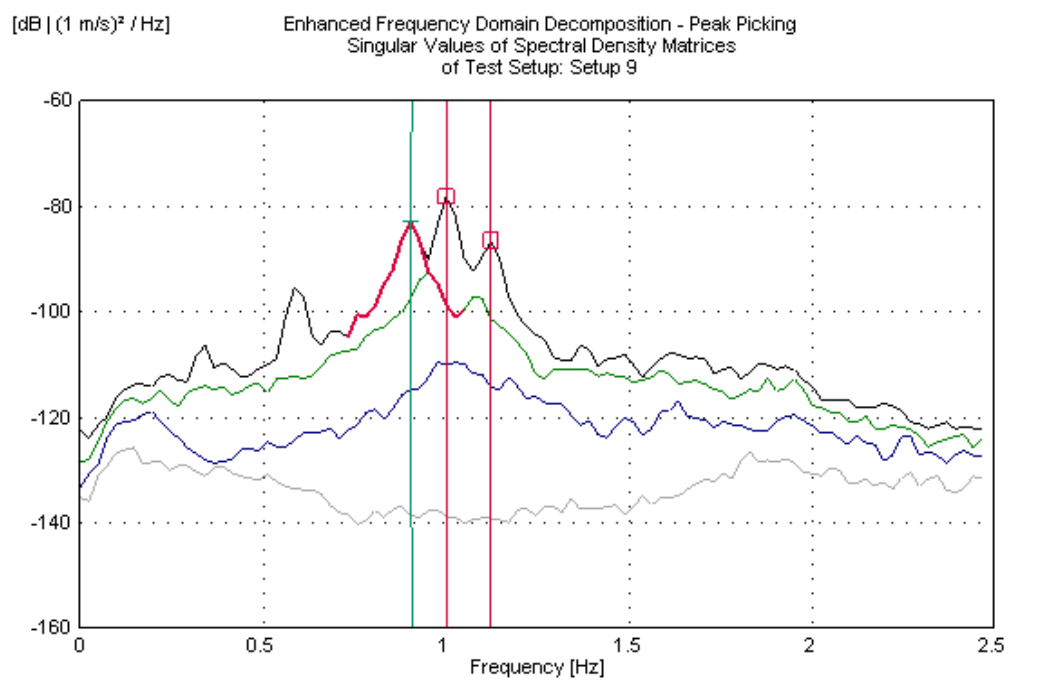

Cursor Values

Frequency $=0.9069 \mathrm{~Hz}$ SVD Line =

Estimation Parameters $\mathrm{t}=0.9034 \pm 0.000004196 \mathrm{H} \bar{z}$

$z=2.019 \pm 0.01736 \%$

MAC Level $=0.6$

Indicators

ㄴstimated Mode

+ Selected Mode

Lines

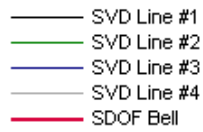

[Correlation] Normalized Correlation Function of Singular Value Spectral Bell for Test Setup: Setup 9

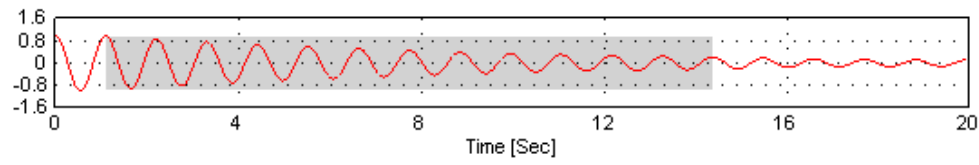

Cursor Values

$X=$

Correlation Limits

$\operatorname{Min}=0.2$

$\operatorname{Max}=0.95$

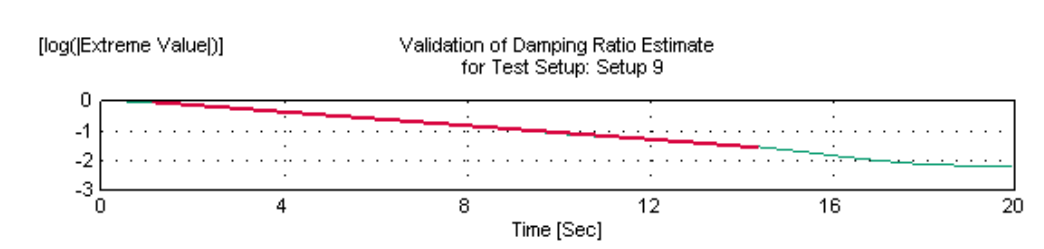

Cursor Values

$X=$

Estimation Parameters

$z=2.019 \pm 0.01736 \%$

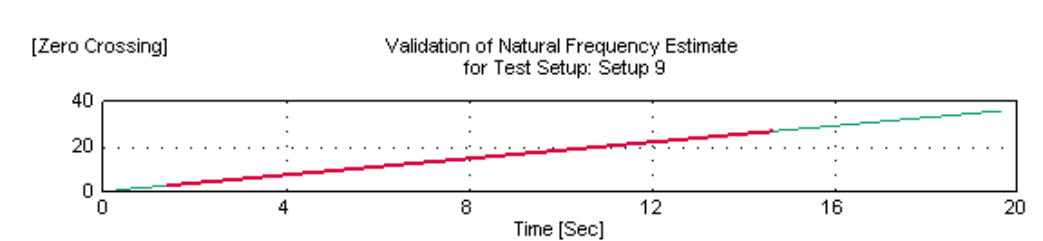

Cursor Values

$X=$
$Y=$

Estimation Parameters $t=0.903 \pm 0.0000042 \mathrm{~Hz}$ 


\section{Setup 10:}

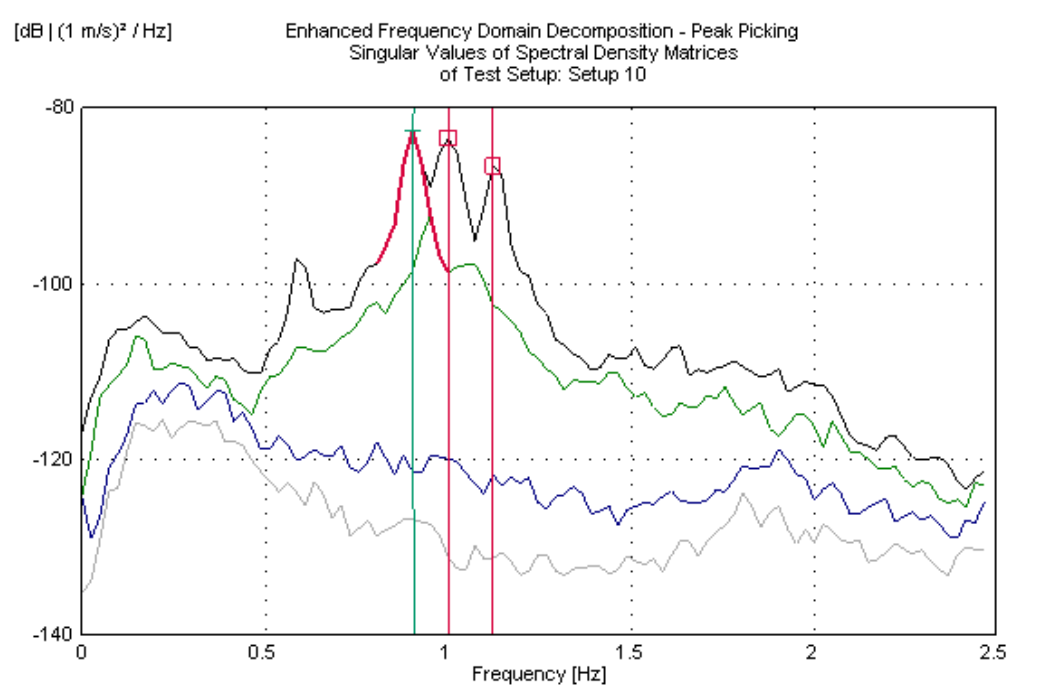

\author{
Cursor Values \\ Frequency $=0.9069 \mathrm{~Hz}$ \\ SVD Line =
}

Estimation Parameters $\mathrm{f}=0.9031 \pm 0.000008908 \mathrm{H} \bar{z}$ $z=2.06 \pm 0.03528 \%$ MAC Level $=0.91$

Indicators

ㄴ Estimated Mode + Selected Mode

Lines

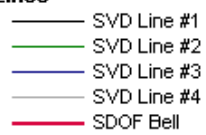

[Correlation] Normalized Correlation Function of Singular Value Spectral Bell for Test Setup: Setup 10

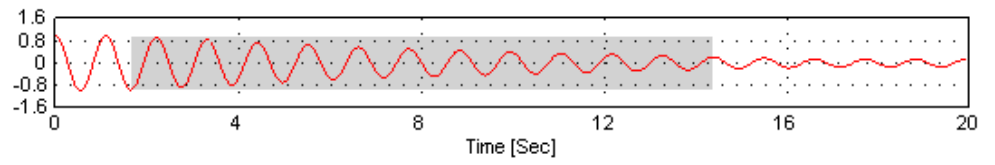

Cursor Values

$X=$

Correlation Limits

Min $=0.2$

$\operatorname{Max}=0.95$

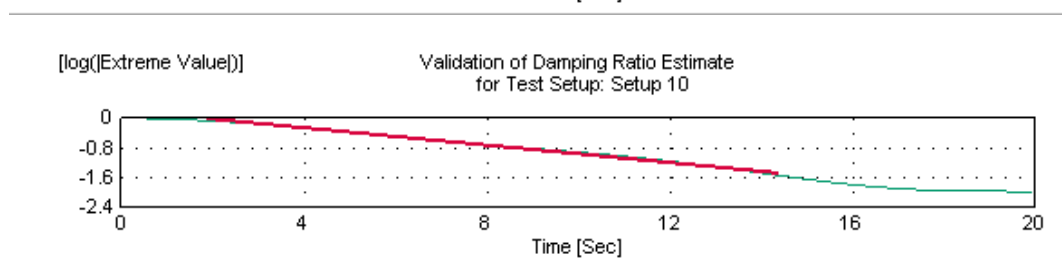

Cursor Values

$X=$

Estimation Parameters $z=2.06 \pm 0.03528 \%$

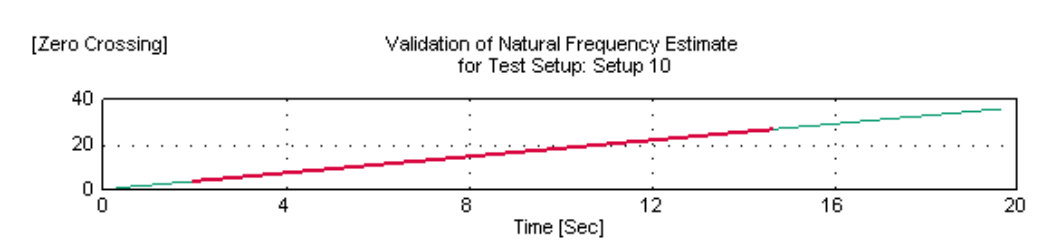

\section{Cursor Values}

$X=$
$Y=$

Estimation Parameters $t=0.903 \pm 0.00000891 \mathrm{~Hz}$ 


\section{Setup 11:}

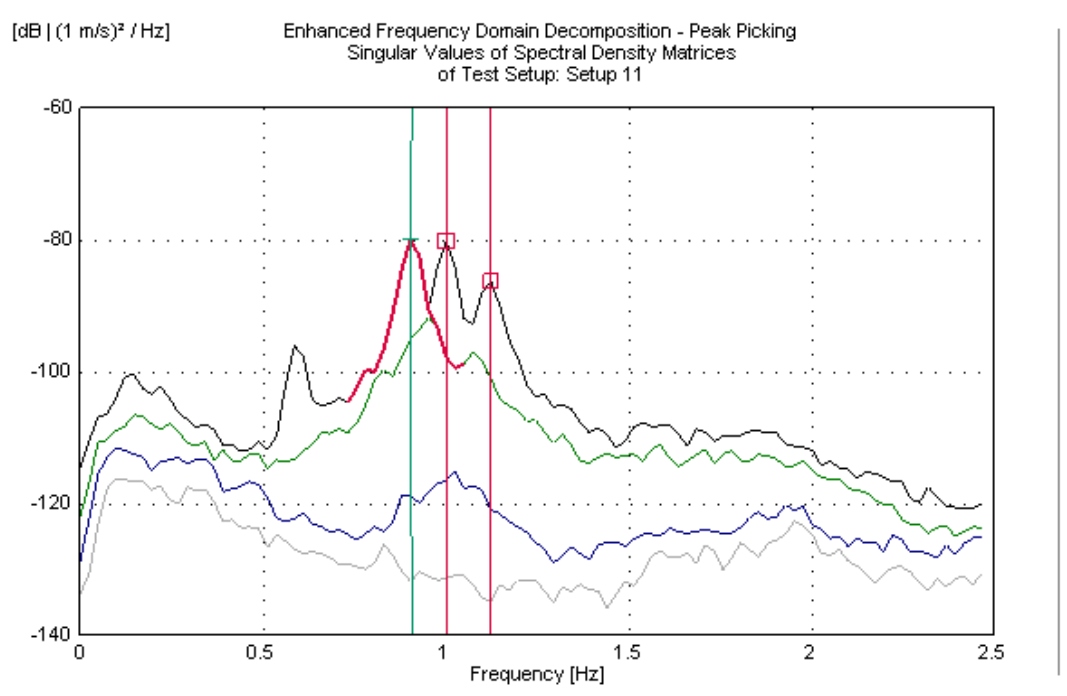

Cursor Values

Frequency $=0.9069 \mathrm{~Hz}$ SVD Line =

Estimation Parameters $\mathrm{t}=0.9072 \pm 0.000008207 \mathrm{H}_{2}$

$z=2.037 \pm 0.03943 \%$

MAC Level $=0.782$

Indicators

ㄴstimated Mode

+ Selected Mode

Lines

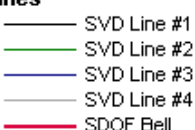

[Correlation] Normalized Correlation Function of Singular Value Spectral Bell for Test Setup: Setup 11

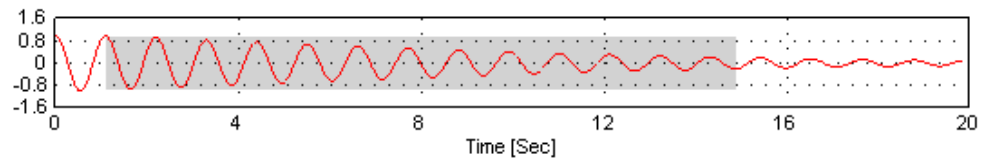

Cursor Values

$X=$

Correlation Limits

$\operatorname{Min}=0.2$

$\operatorname{Max}=0.95$

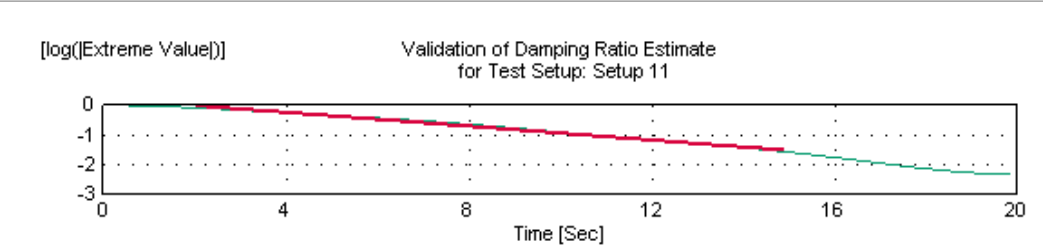

Cursor Values

$X=$

Estimation Parameters

$z=2.037 \pm 0.03943 \%$

[Zero Crossing] Validation of Natural Frequency Estimate

for Test Setup: Setup 11

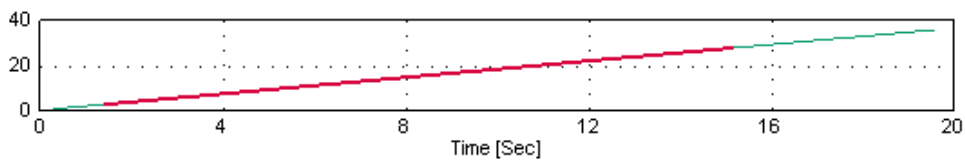

Cursor Values

$X=$
$Y=$

Estimation Parameters $f=0.907 \pm 0.00000821 \mathrm{~Hz}$ 


\section{Setup 12:}

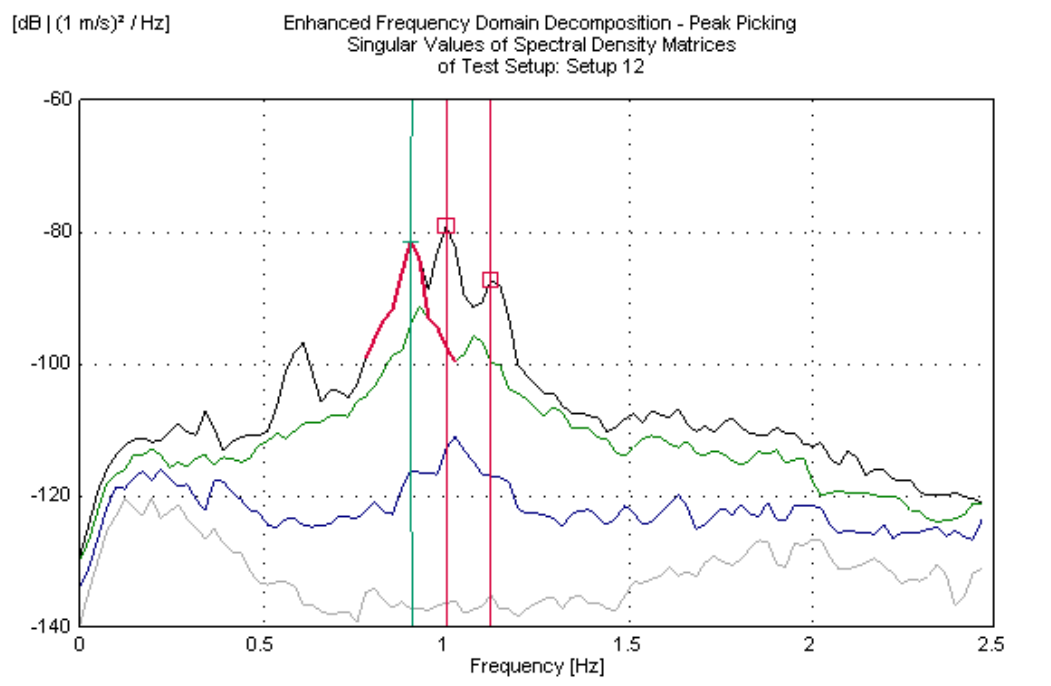

Cursor Values

Frequency $=0.9069 \mathrm{~Hz}$ SVD Line =

Estimation Parameters $f=0.9081 \pm 0.000006563 \mathrm{H}_{2}$

$z=1.851 \pm 0.03416 \%$

MAC Level $=0.56$

Indicators

$\square$ Estimated Mode

+ Selected Mode

Lines

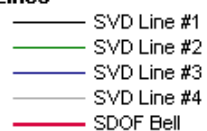

[Correlation] Normalized Correlation Function of Singular Value Spectral Bell for Test Setup: Setup 12

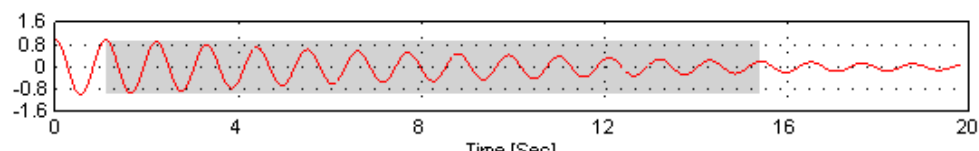

Cursor Values

$X=$

Correlation Limits

$\operatorname{Min}=0.2$

$\operatorname{Max}=0.95$

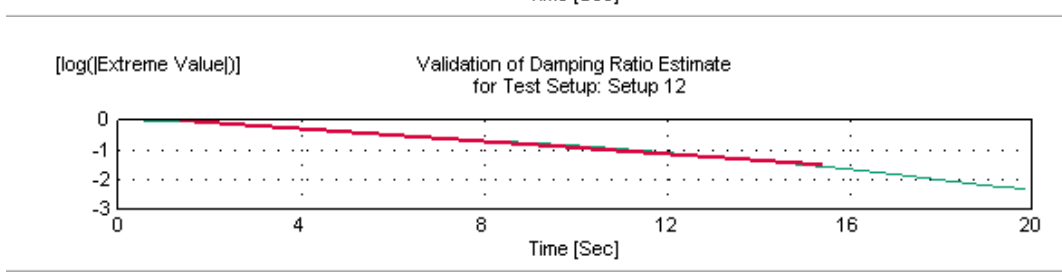

Cursor Values

$X=$

Estimation Parameters

$z=1.851 \pm 0.03416 \%$

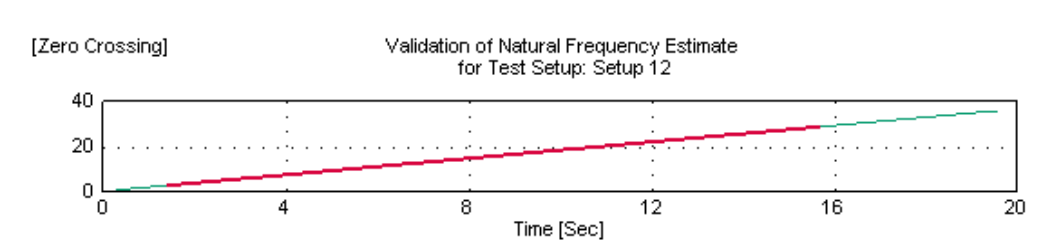

Cursor Values

$X=$
$Y=$

Estimation Parameters $f=0.908 \pm 0.00000656 \mathrm{~Hz}$ 


\section{Setup 13:}

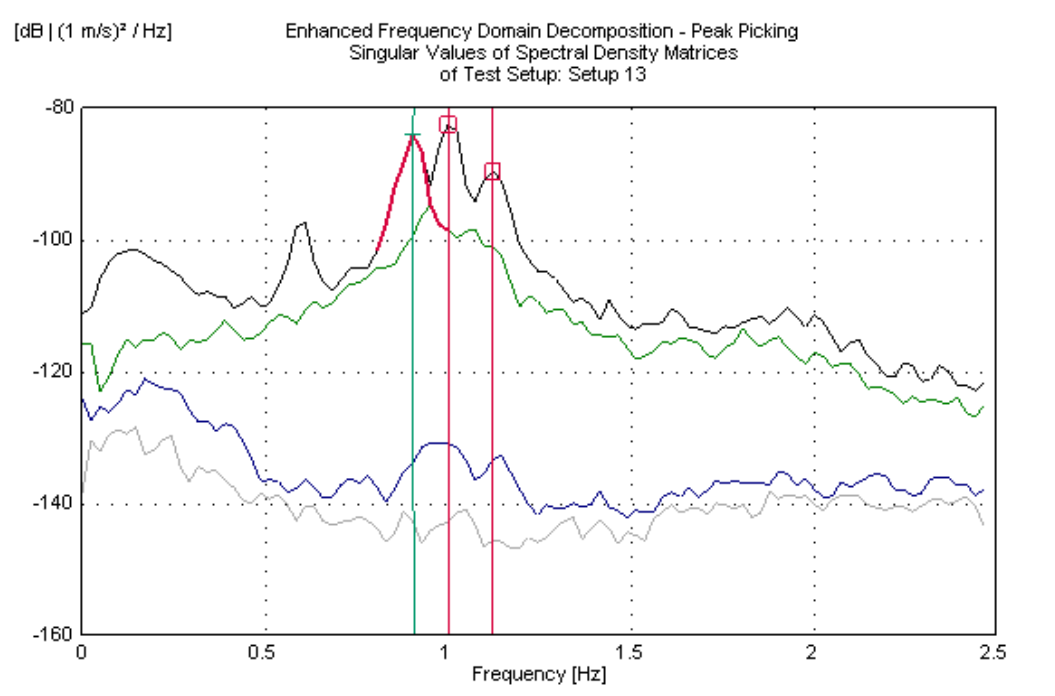

\author{
Cursor Values \\ Frequency $=0.9069 \mathrm{~Hz}$ \\ SVD Line =
}

Estimation Parameters $f=0.9106 \pm 0.00001928 \mathrm{~Hz}$ $z=2.208 \pm 0.01836 \%$ MAC Level $=0.7$

Indicators

ㅁ Estimated Mode + Selected Mode

Lines

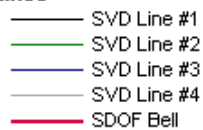

[Correlation] Normalized Correlation Function of Singular Value Spectral Bell for Test Setup: Setup 13

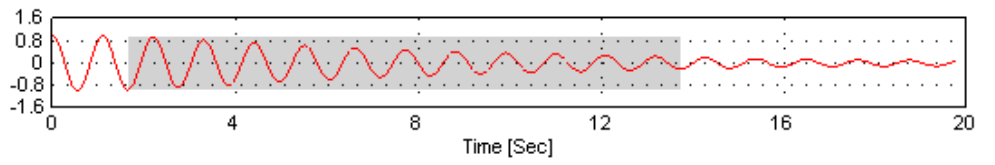

[log(|Extreme Value|) $]$

Validation of Damping Ratio Estimate

for Test Setup: Setup 13

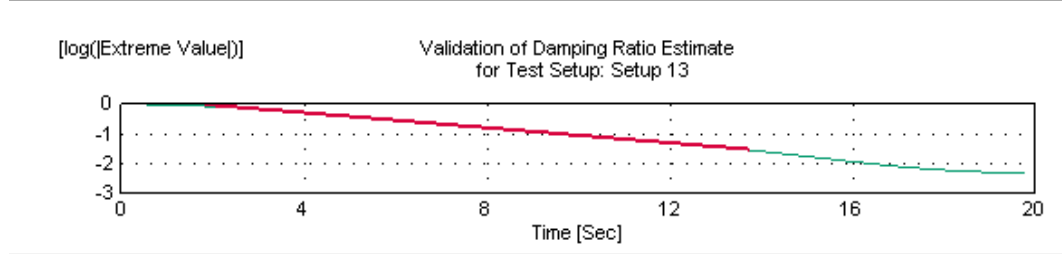

Cursor Values

$X=$

Correlation Limits

$\operatorname{Min}=0.2$

$\operatorname{Max}=0.95$

Cursor Values

$X=$

Estimation Parameters

$z=2.208 \pm 0.01836 \%$

[Zero Crossing] Validation of Natural Frequency Estimate

for Test Setup: Setup 13

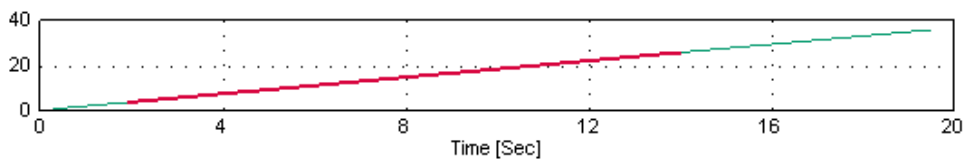

Cursor Values

$X=$
$Y=$

Estimation Parameters $t=0.911 \pm 0.0000193 \mathrm{~Hz}$ 


\section{Setup 14:}

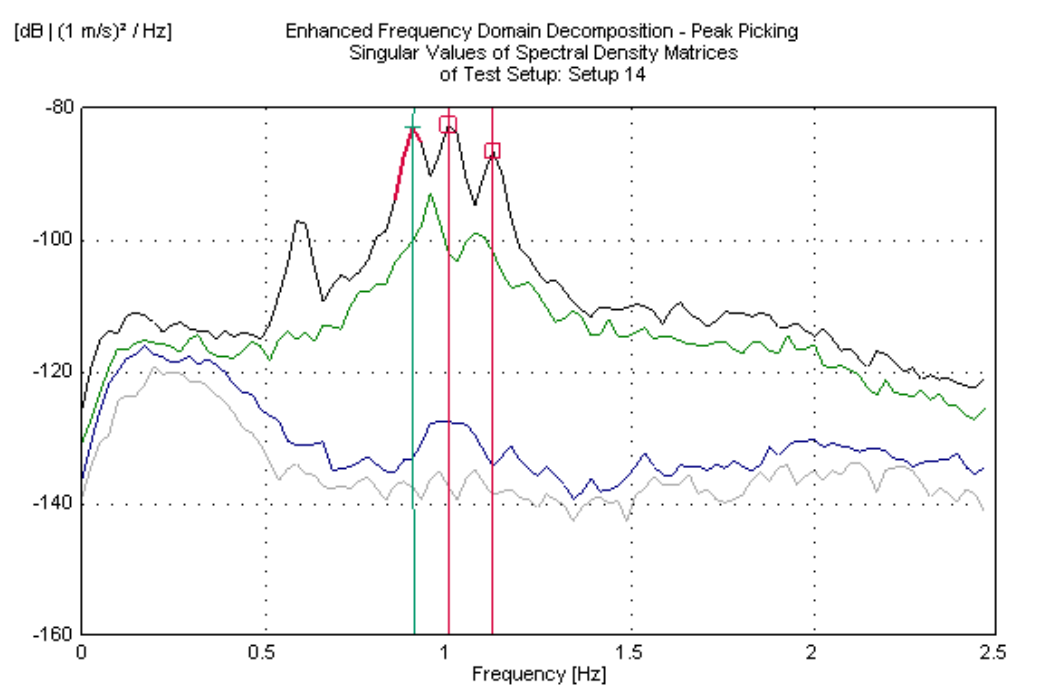

Cursor Values

Frequency $=0.9069 \mathrm{~Hz}$ SVD Line $=$

Estimation Parameters $f=0.9113 \pm 0.00002041 \mathrm{~Hz}$ $z=2.188 \pm 0.07034 \%$ MAC Level $=0.6119$

Indicators

ㄴ Estimated Mode + Selected Mode

Lines

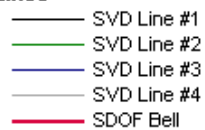

[Correlation] Normalized Correlation Function of Singular Value Spectral Bell for Test Setup: Setup 14

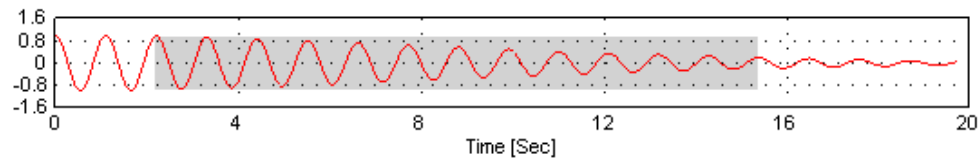

$[\log (\mid$ Extreme Value $)]$

Validation of Damping Ratio Estimate

for Test Setup: Setup 14

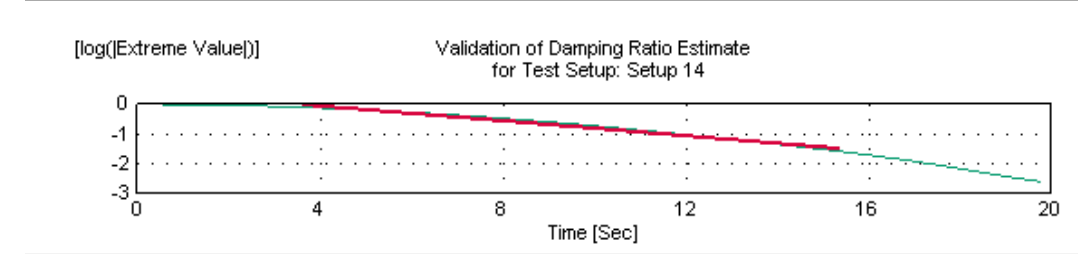

Cursor Values

$X=$

Correlation Limits

$\operatorname{Min}=0.2$

$\operatorname{Max}=0.95$

Cursor Values

$X=$

Estimation Parameters

$z=2.188 \pm 0.07034 \%$

[Zero Crossing] Validation of Natural Frequency Estimate

for Test Setup: Setup 14

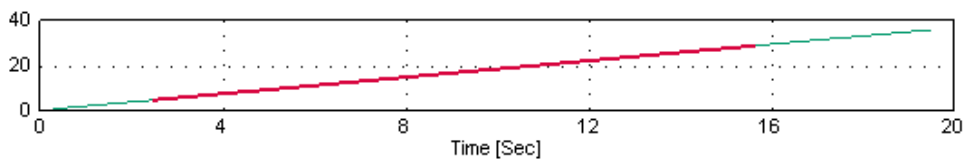

Cursor Values

$X=$
$Y=$

Estimation Parameters $t=0.911 \pm 0.0000204 \mathrm{~Hz}$ 


\section{Setup 15:}

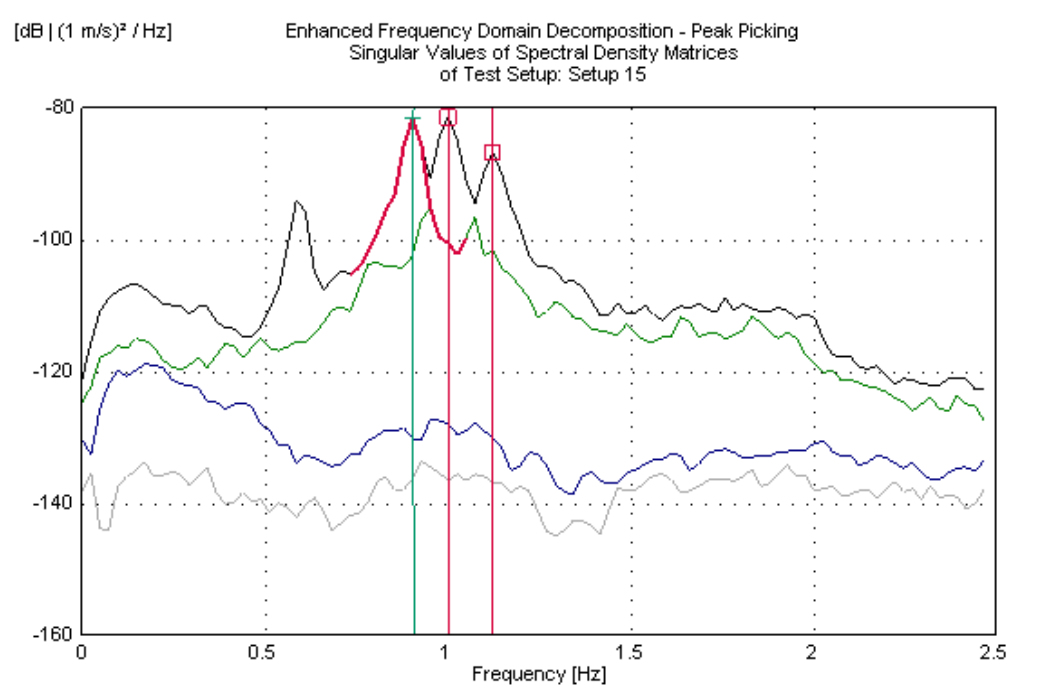

\author{
Cursor Values \\ Frequency $=0.9069 \mathrm{~Hz}$ \\ SVD Line $=$
}

Estimation Parameters $\mathrm{t}=0.9046 \pm 0.000006927 \mathrm{H}_{2}$ $z=1.788 \pm 0.03754 \%$ MAC Level $=0.75$

Indicators

ㅁ Estimated Mode + Selected Mode

Lines

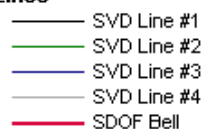

[Correlation] Normalized Correlation Function of Singular Value Spectral Bell for Test Setup: Setup 15

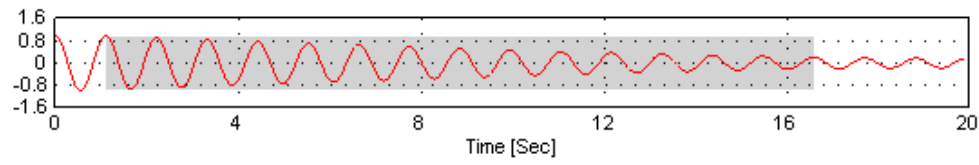

[log(|Extreme Value $)] \quad$ Validation of Damping Ratio Estimate

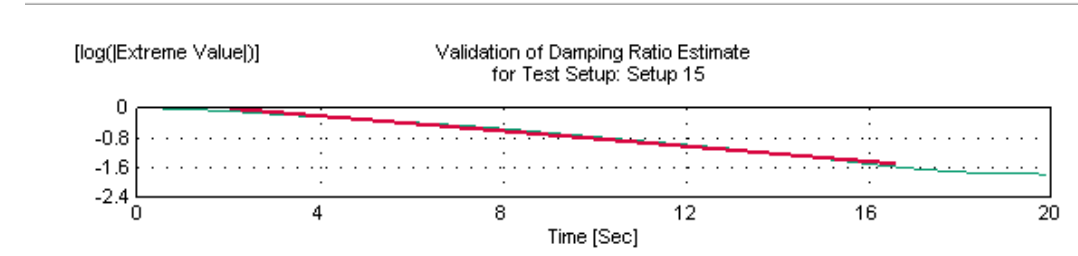

Cursor Values

$X=$

Correlation Limits

Min $=0.2$

$\operatorname{Max}=0.95$

Cursor Values

$X=$

Estimation Parameters $z=1.788 \pm 0.03754 \%$

[Zero Crossing] Validation of Natural Frequency Estimate

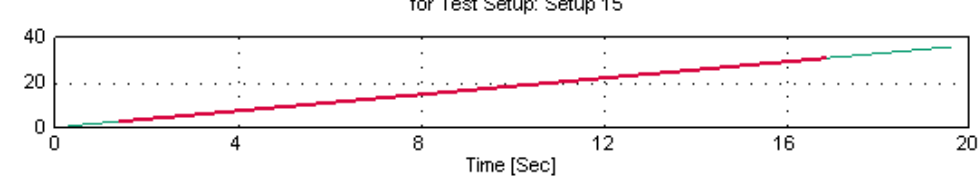

Cursor Values

$X=$
$Y=$

Estimation Parameters $f=0.905 \pm 0.00000693 \mathrm{~Hz}$ 


\section{Setup 16:}
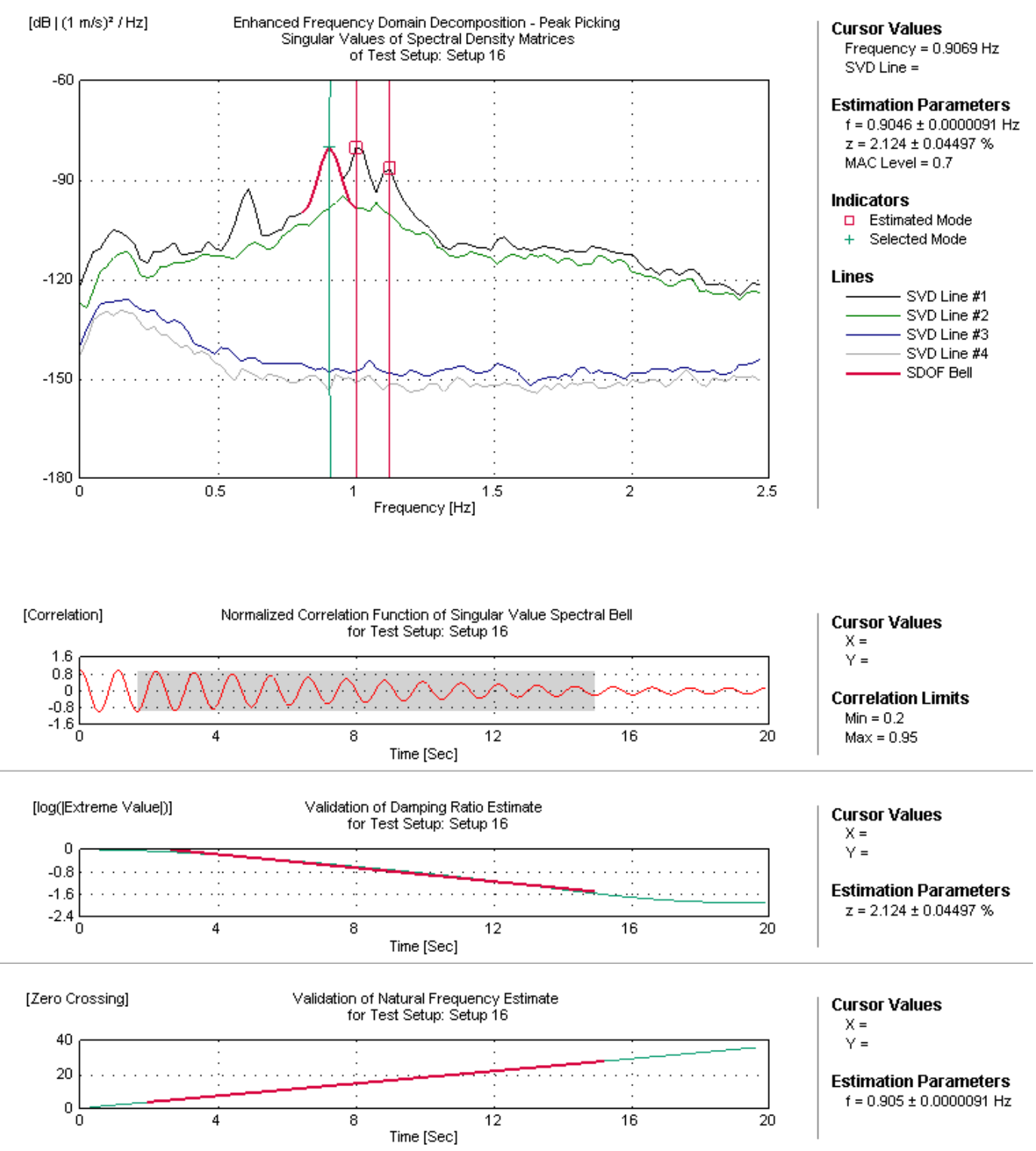


\section{Setup 17:}

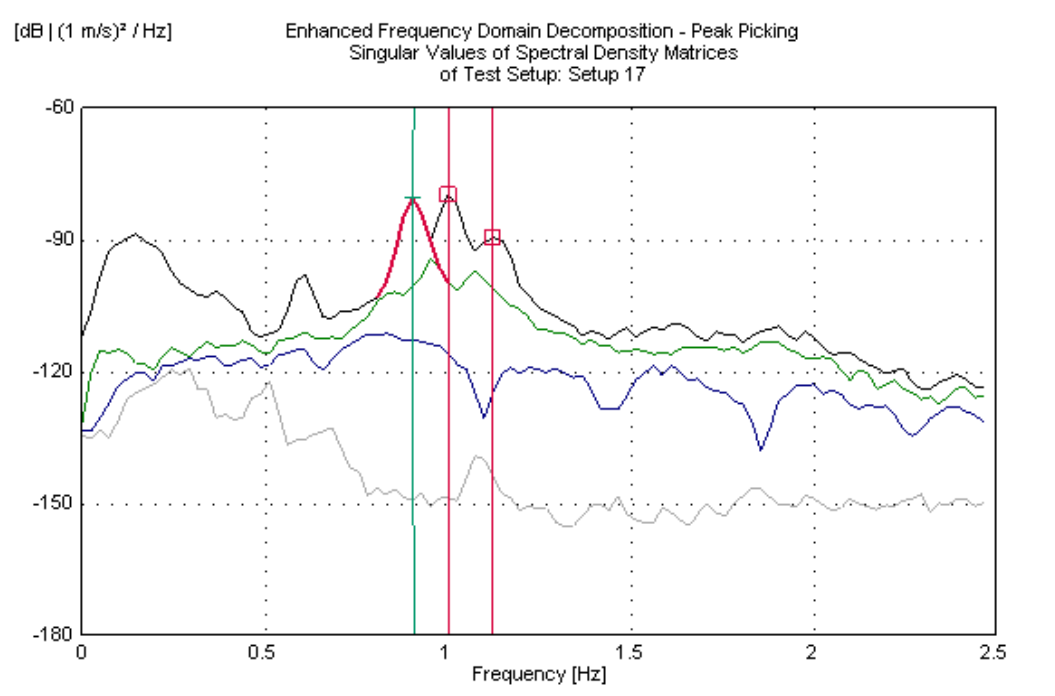

Cursor Values

Frequency $=0.9069 \mathrm{~Hz}$ SVD Line $=$

Estimation Parameters $\mathrm{t}=0.9028 \pm 0.000008384 \mathrm{H}_{2}$ $z=2.088 \pm 0.03745 \%$ MAC Level $=0.55$

Indicators

ㅁ Estimated Mode + Selected Mode

Lines

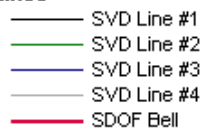

[Correlation] Normalized Correlation Function of Singular Value Spectral Bell for Test Setup: Setup 17

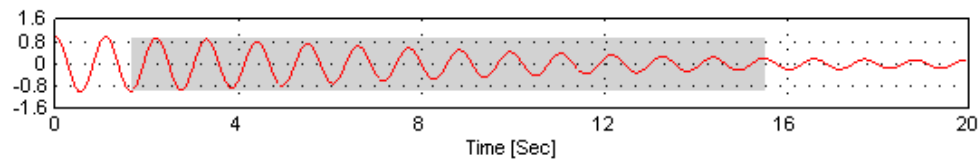

$[\log (\mid$ Extreme Value|)]

Validation of Damping Ratio Estimate

for Test Setup: Setup 17

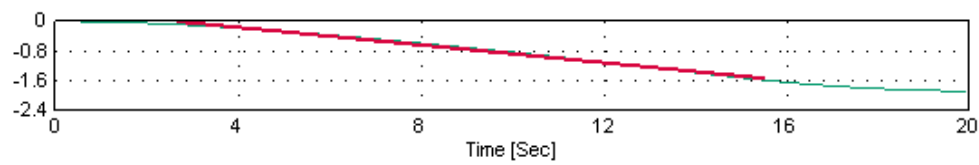

Cursor Values

$X=$

Correlation Limits

$\operatorname{Min}=0.2$

$\operatorname{Max}=0.95$
Cursor Values

$X=$

Estimation Parameters $z=2.088 \pm 0.03745 \%$
[Zero Crossing] Validation of Natural Frequency Estimate

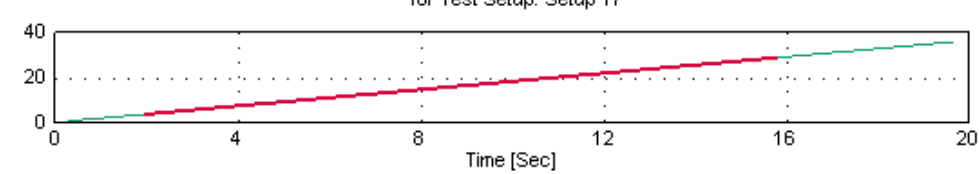

Cursor Values

$X=$
$Y=$

Estimation Parameters $f=0.903 \pm 0.00000838 \mathrm{~Hz}$ 


\section{Setup 18:}

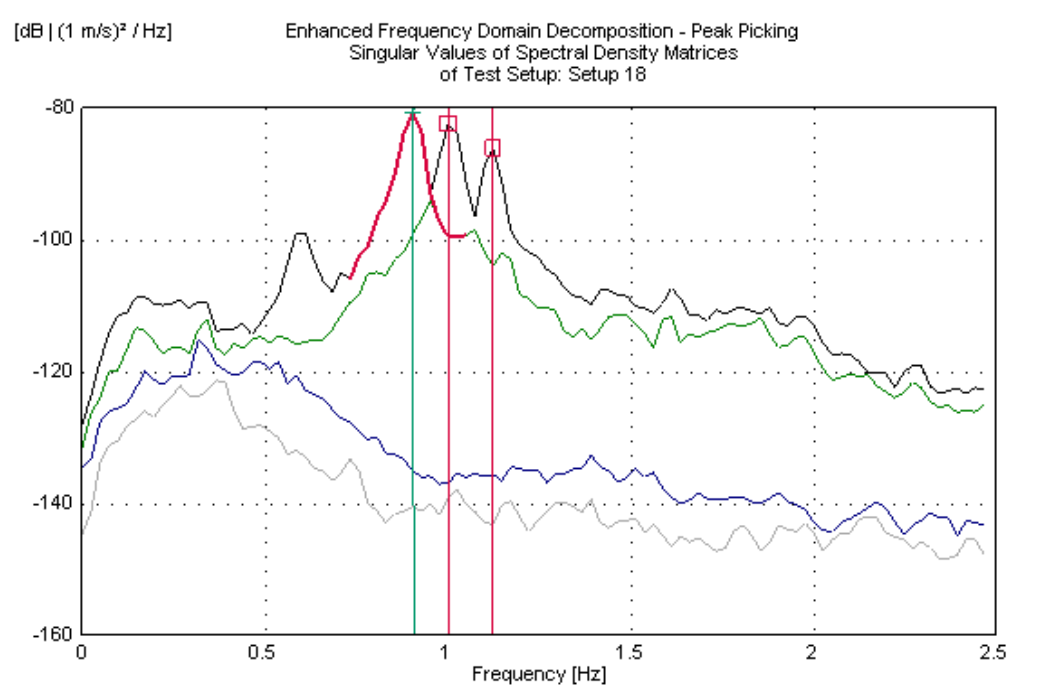

\author{
Cursor Values \\ Frequency $=0.9069 \mathrm{~Hz}$ \\ SVD Line $=$
}

Estimation Parameters $f=0.9061 \pm 0.00001434 \mathrm{~Hz}$ $z=2.249 \pm 0.06237 \%$ MAC Level $=0.8004$

Indicators

ㅁ Estimated Mode + Selected Mode

Lines

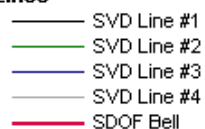

[Correlation] Normalized Correlation Function of Singular Value Spectral Bell for Test Setup: Setup 18

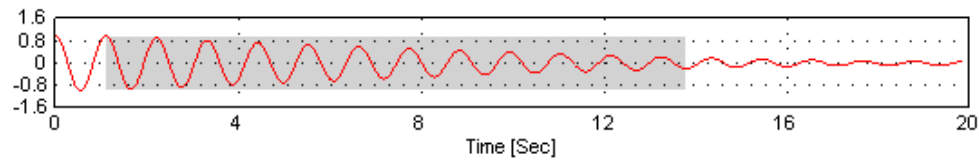

$[\log (\mid$ Extreme Value $)]$

Validation of Damping Ratio Estimate

for Test Setup: Setup 18

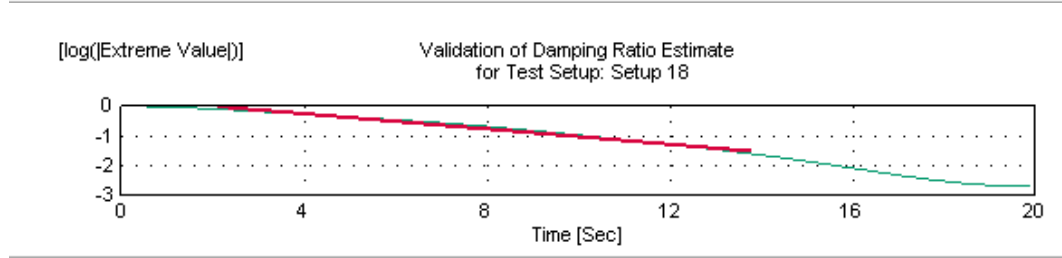

Cursor Values

$X=$

Correlation Limits

$\operatorname{Min}=0.2$

$\operatorname{Max}=0.95$

Cursor Values

$X=$

Estimation Parameters $z=2.249 \pm 0.06237 \%$

[Zero Crossing] Validation of Natural Frequency Estimate for Test Setup: Setup 18

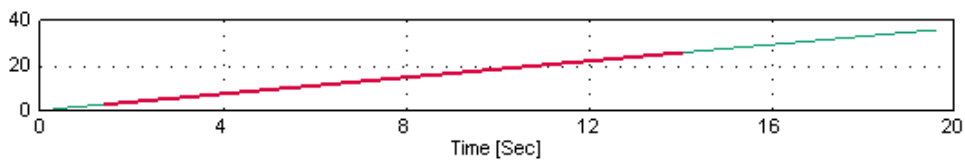

Estimation Parameters $t=0.906 \pm 0.0000143 \mathrm{~Hz}$ 


\section{Identification of Mode 2:}

\section{Setup 1:}
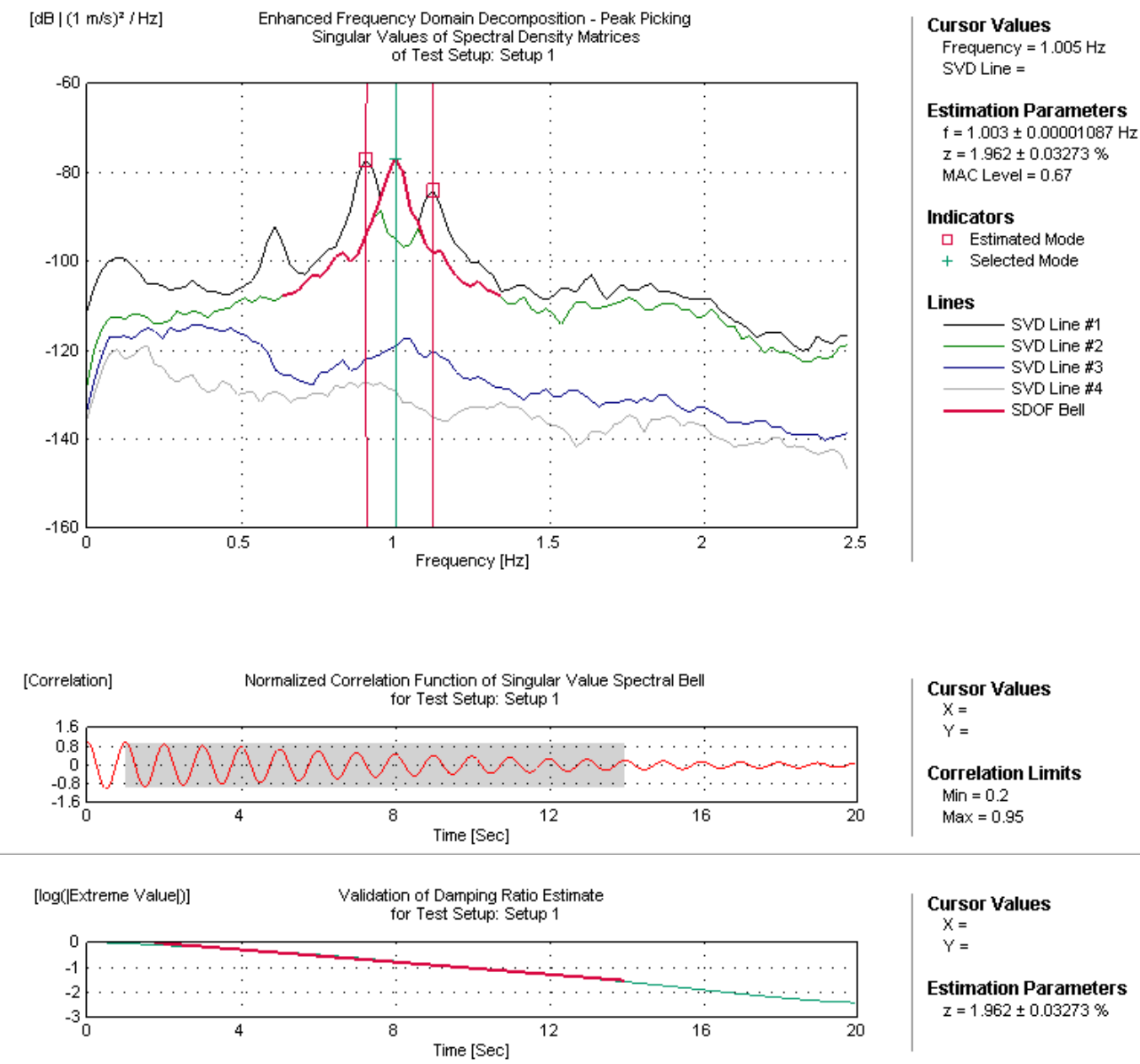

\section{Cursor Values}

$X=$
$Y=$

Estimation Parameters $z=1.962 \pm 0.03273 \%$

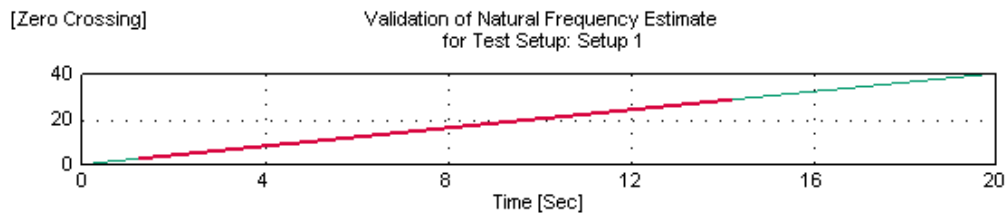

Cursor Values

$X=$

Estimation Parameters

$f=1.003 \pm 0.00001087 \mathrm{~Hz}$ 


\section{Setup 2:}

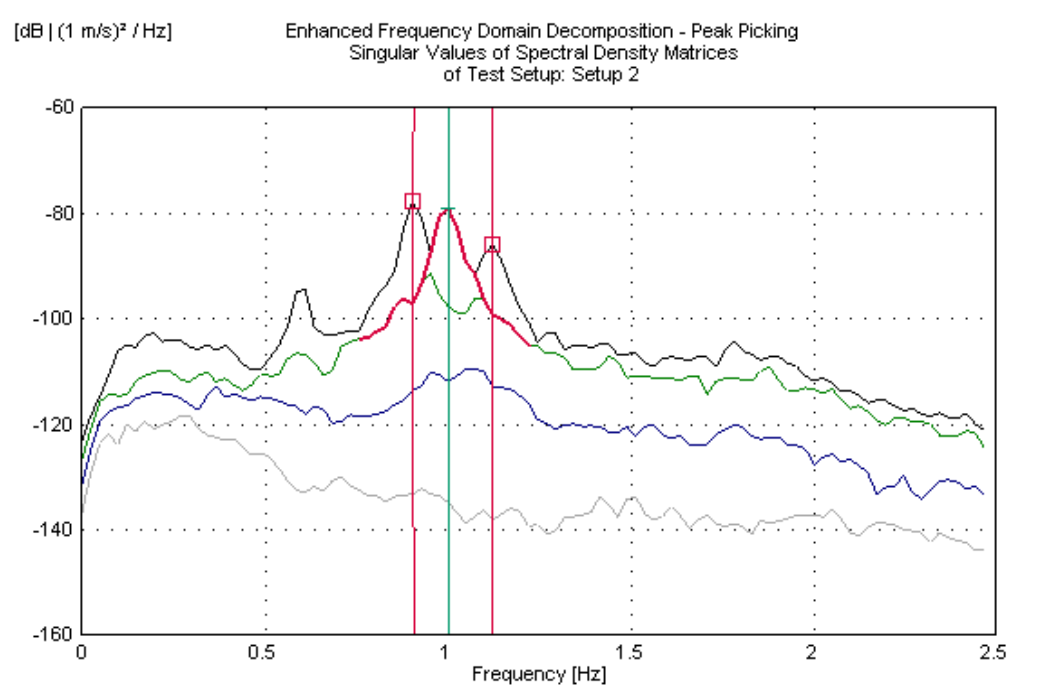

\author{
Cursor Values \\ Frequency $=1.005 \mathrm{~Hz}$ \\ SVD Line $=$
}

Estimation Parameters $f=0.9944 \pm 0.00001491 \mathrm{~Hz}$ $z=2.164 \pm 0.06031 \%$ MAC Level $=0.7$

Indicators

ㅁ Estimated Mode + Selected Mode

Lines

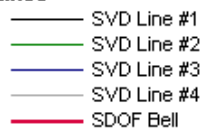

[Correlation] Normalized Correlation Function of Singular Value Spectral Bell for Test Setup: Setup 2

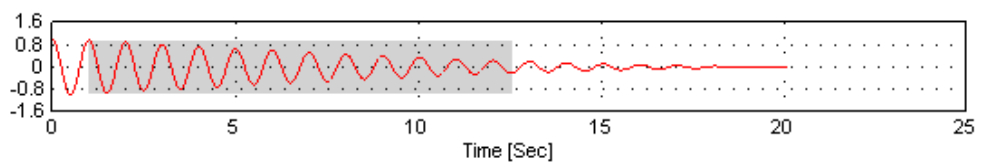

$[\log (\mid$ Extreme Value $\mid)]$

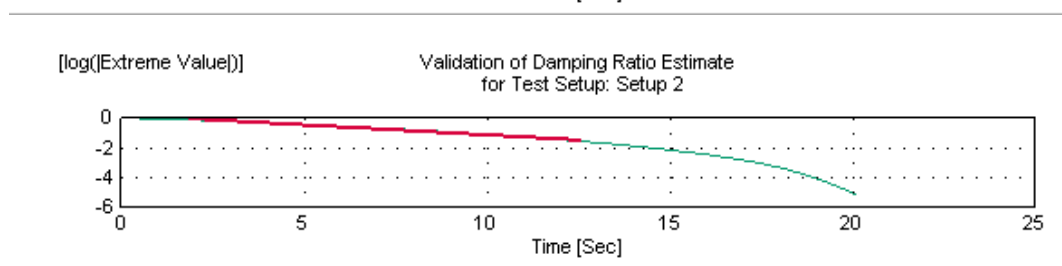

Cursor Values

$X=$

Correlation Limits

Min $=0.2$

$\operatorname{Max}=0.95$

Cursor Values

$X=$

Estimation Parameters $z=2.164 \pm 0.06031 \%$

[Zero Crossing] Validation of Natural Frequency Estimate for Test Setup: Setup 2

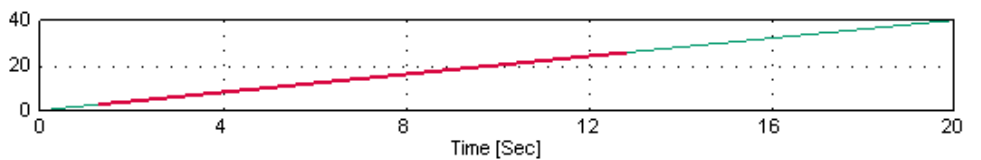

Estimation Parameters $t=0.9944 \pm 0.00001491 \mathrm{~Hz}$ 


\section{Setup 3:}

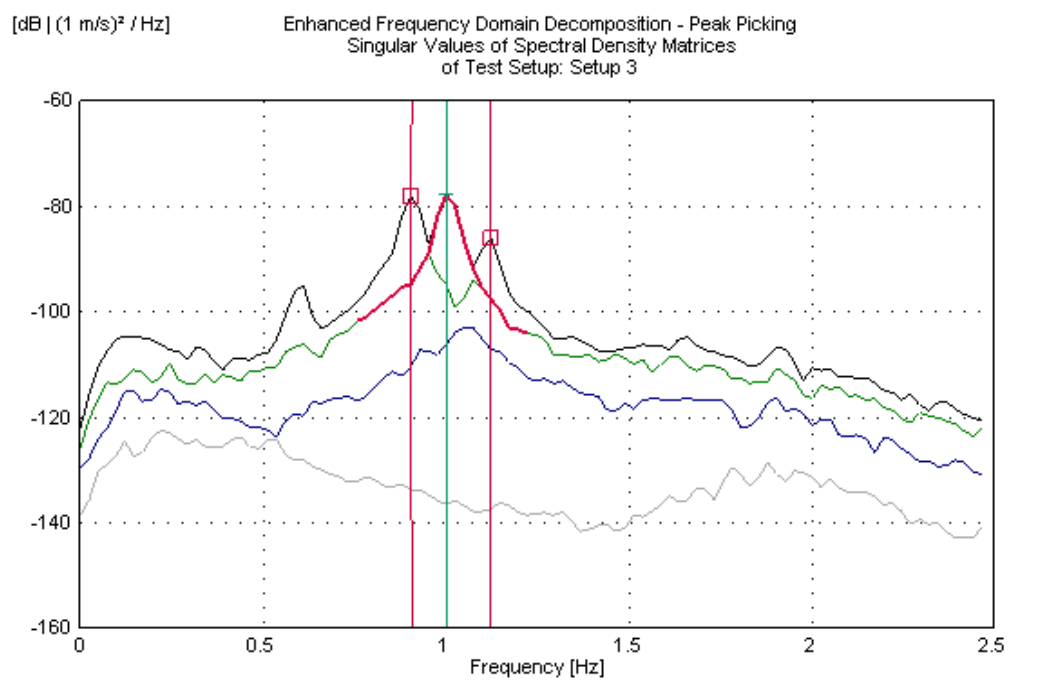

Cursor Values

Frequency $=1.005 \mathrm{~Hz}$ SVD Line =

Estimation Parameters $t=1.005 \pm 0.00001132 \mathrm{~Hz}$

$z=2.012 \pm 0.0498 \%$

MAC Level $=0.7$

Indicators

ㄴstimated Mode

+ Selected Mode

Lines

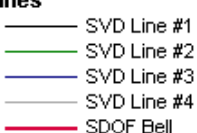

[Correlation] Normalized Correlation Function of Singular Value Spectral Bell for Test Setup: Setup 3

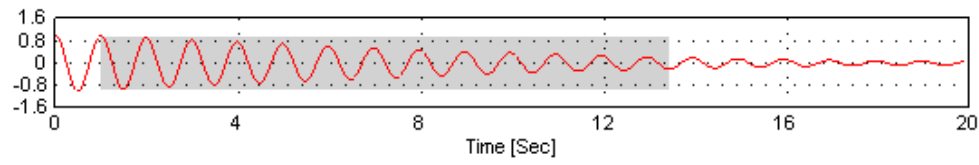

Cursor Values

$X=$

Correlation Limits

Min $=0.2$

$\operatorname{Max}=0.95$

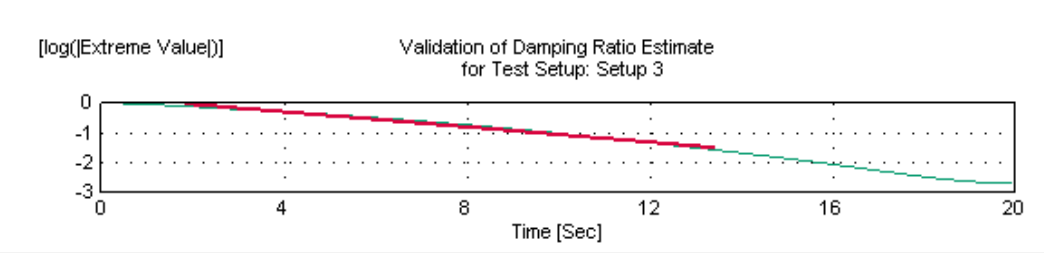

Cursor Values

$X=$

Estimation Parameters

$z=2.012 \pm 0.0498 \%$

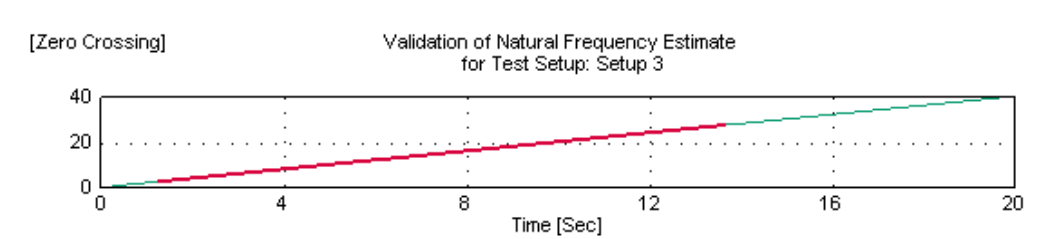

Cursor Values

$X=$
$Y=$

Estimation Parameters $f=1.005 \pm 0.00001132 \mathrm{~Hz}$ 


\section{Setup 4:}

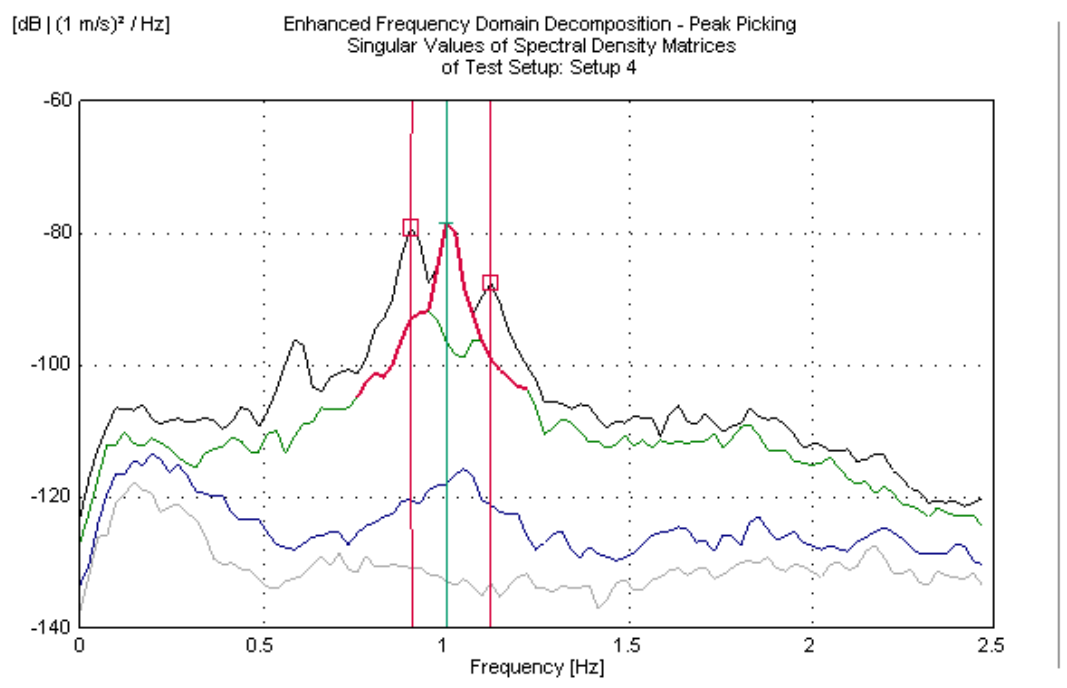

Cursor Values

Frequency $=1.005 \mathrm{~Hz}$ SVD Line =

Estimation Parameters $t=1.009 \pm 0.00001142 \mathrm{~Hz}$

$z=1.678 \pm 0.05304 \%$

MAC Level $=0.8$

Indicators

$\square$ Estimated Mode

+ Selected Mode

Lines

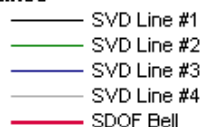

[Correlation] Normalized Correlation Function of Singular Value Spectral Bell for Test Setup: Setup 4

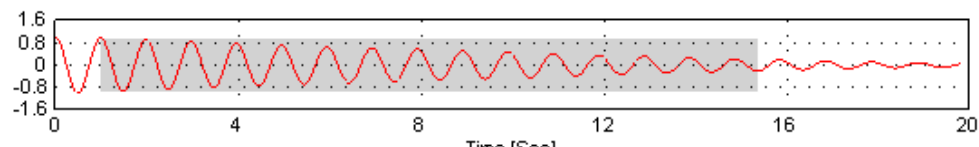

Time [Sec]

for Test Setup: Setup 4

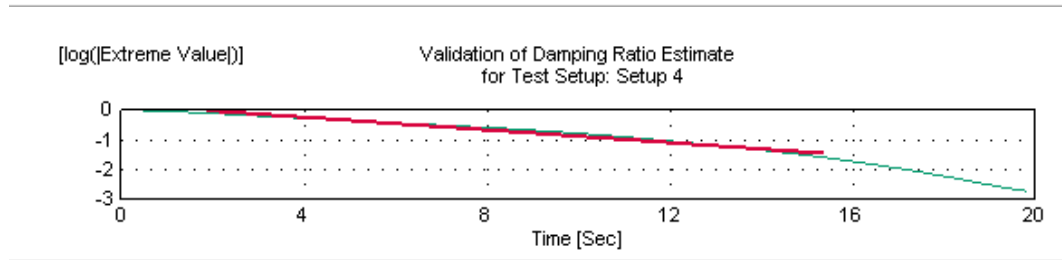

Cursor Values

$X=$

Correlation Limits

$\operatorname{Min}=0.2$

$\operatorname{Max}=0.95$

Cursor Values

$X=$

Estimation Parameters $z=1.678 \pm 0.05304 \%$

[Zero Crossing] Validation of Natural Frequency Estimate

for Test Setup: Setup 4

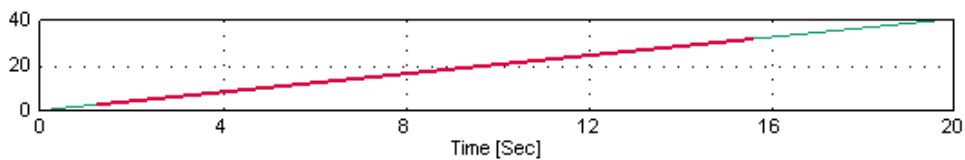

Cursor Values

$X=$
$Y=$

Estimation Parameters $t=1.009 \pm 0.00001142 \mathrm{~Hz}$ 


\section{Setup 5:}

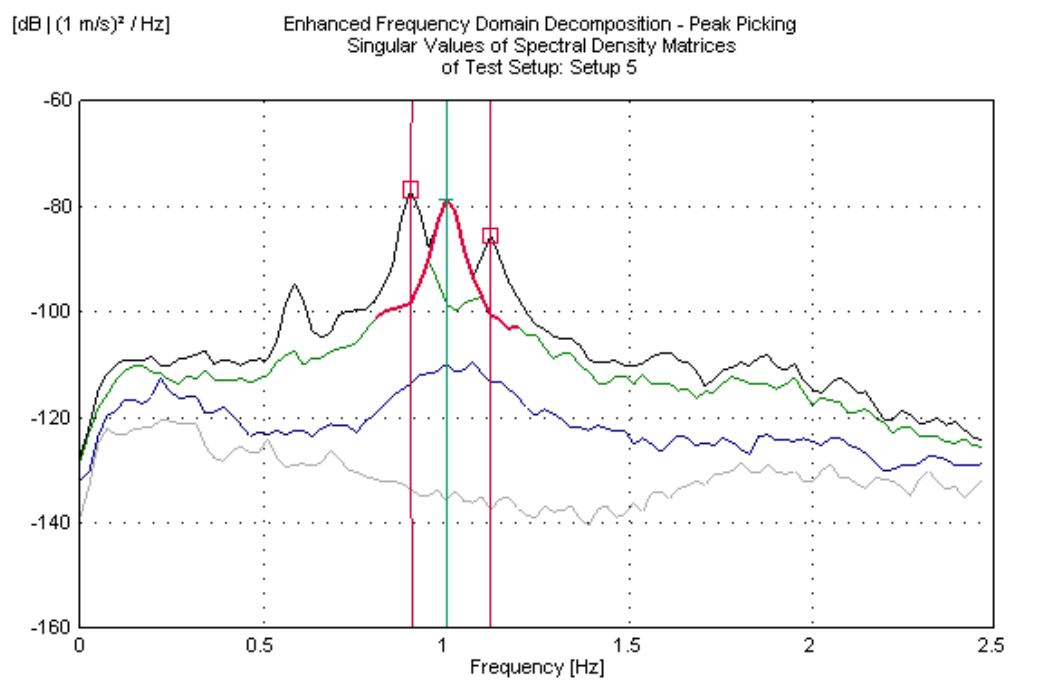

Cursor Values

Frequency $=1.005 \mathrm{~Hz}$ SVD Line =

Estimation Parameters $t=1.006 \pm 0.000009949 \mathrm{~Hz}$ $z=1.972 \pm 0.04981 \%$ MAC Level $=0.818$

Indicators

$\square$ Estimated Mode + Selected Mode

Lines

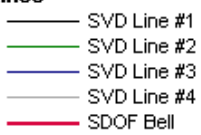

[Correlation] Normalized Correlation Function of Singular Value Spectral Bell for Test Setup: Setup 5

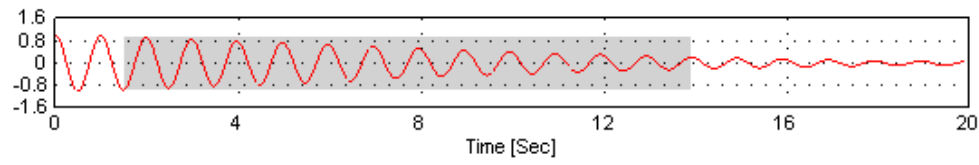

Cursor Values

$X=$

Correlation Limits

$\operatorname{Min}=0.2$

$\operatorname{Max}=0.95$

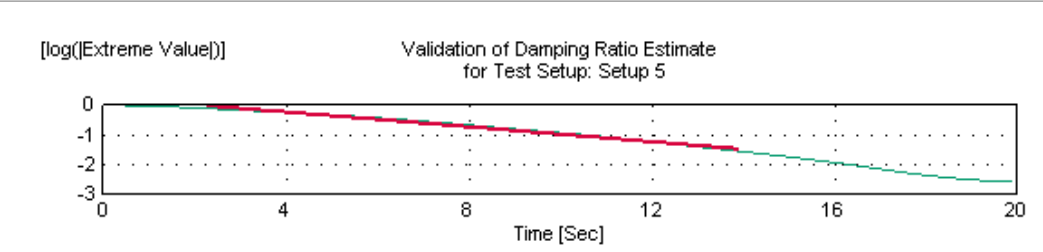

Cursor Values

$X=$

Estimation Parameters $z=1.972 \pm 0.04981 \%$

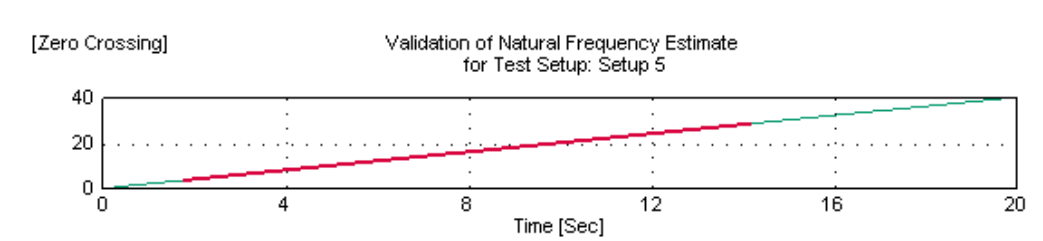

Cursor Values

$X=$
$Y=$

Estimation Parameters $t=1.006 \pm 0.000009949 \mathrm{~Hz}$ 


\section{Setup 6:}

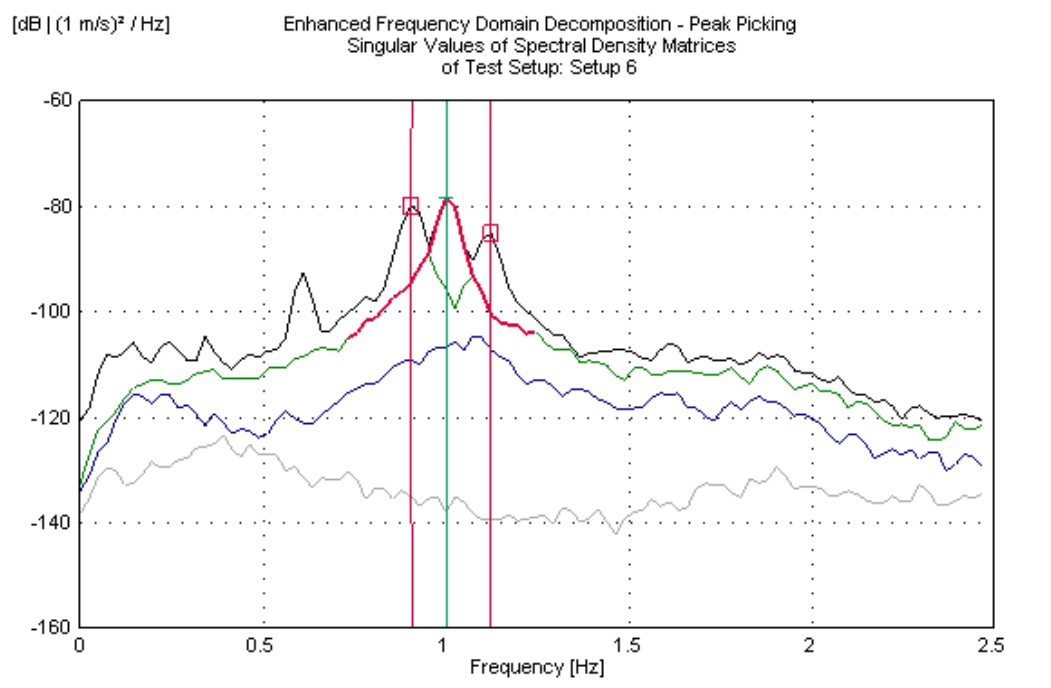

Cursor Values

Frequency $=1.005 \mathrm{~Hz}$

SVD Line =

Estimation Parameters

$f=1.007 \pm 0.000009269 \mathrm{~Hz}$

$z=1.948 \pm 0.04591 \%$

MAC Level $=0.73$

Indicators

$\square$ Estimated Mode

+ Selected Mode

Lines

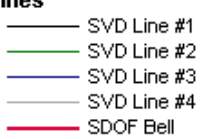

[Correlation] Normalized Correlation Function of Singular Value Spectral Bell for Test Setup: Setup 6

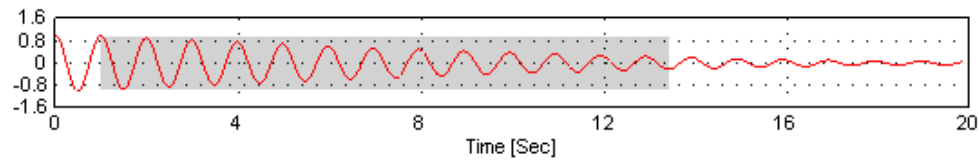

Cursor Values

$\mathrm{X}=$

Correlation Limits

$\operatorname{Min}=0.2$

$\operatorname{Max}=0.95$

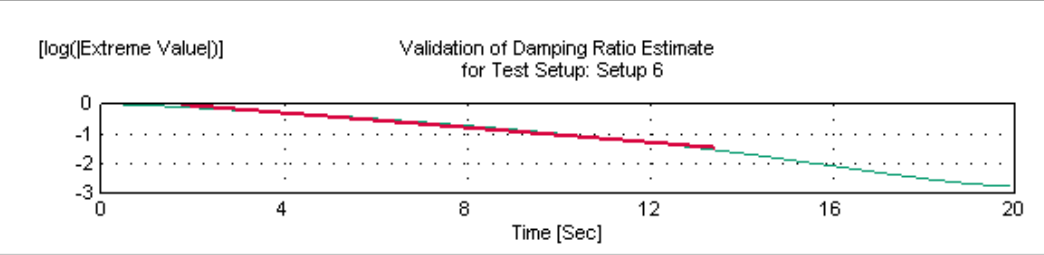

Cursor Values

$X=$

Estimation Parameters $z=1.948 \pm 0.04591 \%$

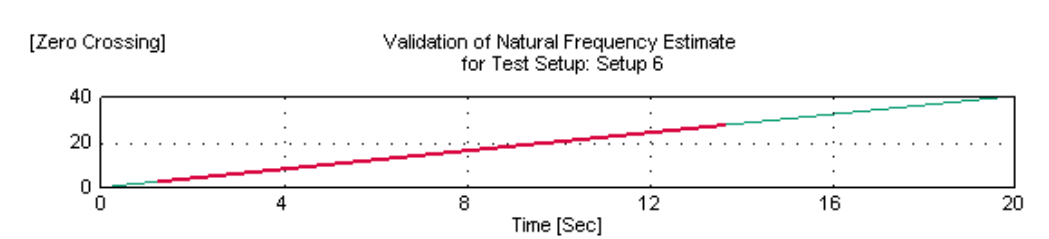

Cursor Values

$X=$
$Y=$

Estimation Parameters $t=1.007 \pm 0.000009269 \mathrm{~Hz}$ 


\section{Setup 7:}

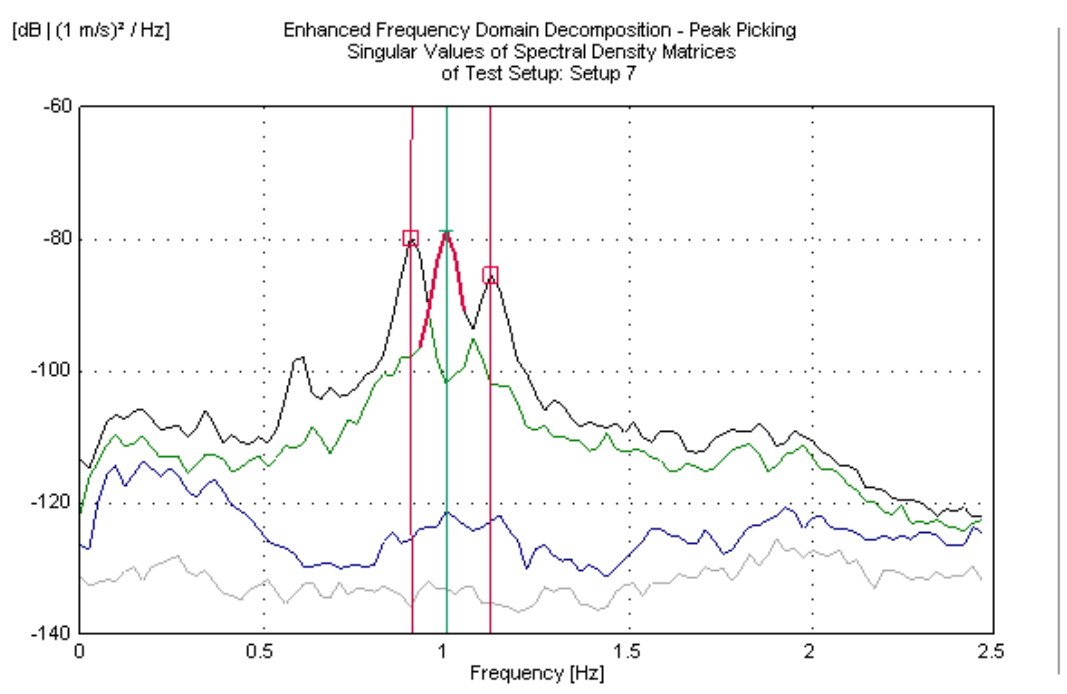

Cursor Values

Frequency $=1.005 \mathrm{~Hz}$ SVD Line =

Estimation Parameters $f=1.002 \pm 0.000008593 \mathrm{~Hz}$

$z=1.803 \pm 0.02864 \%$

MAC Level $=0.62$

Indicators

ㄴstimated Mode

+ Selected Mode

Lines

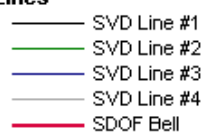

[Correlation] Normalized Correlation Function of Singular Value Spectral Bell for Test Setup: Setup ?

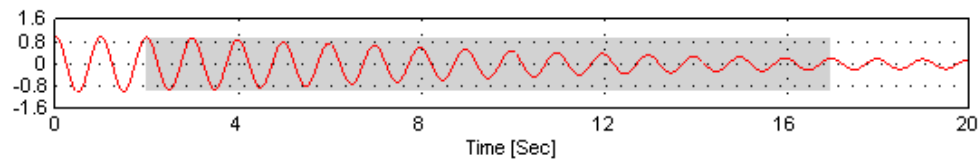

[log(|Extreme Value|)] Validation of Damping Ratio Estimate

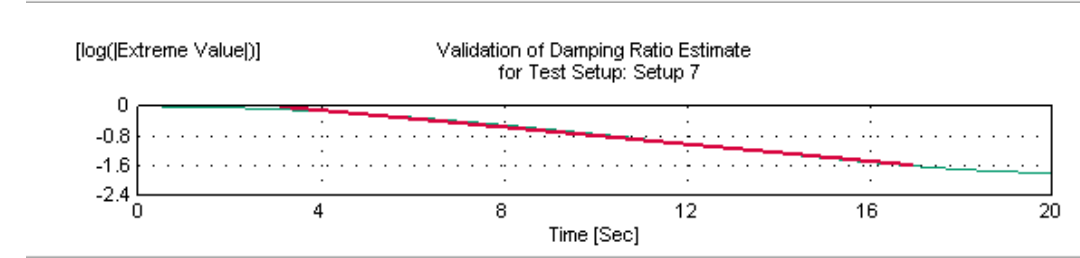

Cursor Values

$X=$

Correlation Limits

Min $=0.2$

$\operatorname{Max}=0.95$

Cursor Values

$X=$

Estimation Parameters

$z=1.803 \pm 0.02864 \%$

[Zero Crossing] Validation of Natural Frequency Estimate

for Test Setup: Setup 7

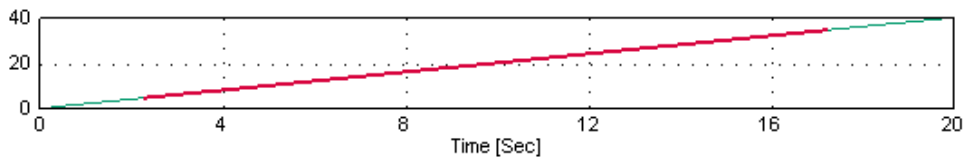

Cursor Values

$X=$
$Y=$

Estimation Parameters $t=1.002 \pm 0.000008593 \mathrm{~Hz}$ 


\section{Setup 8:}
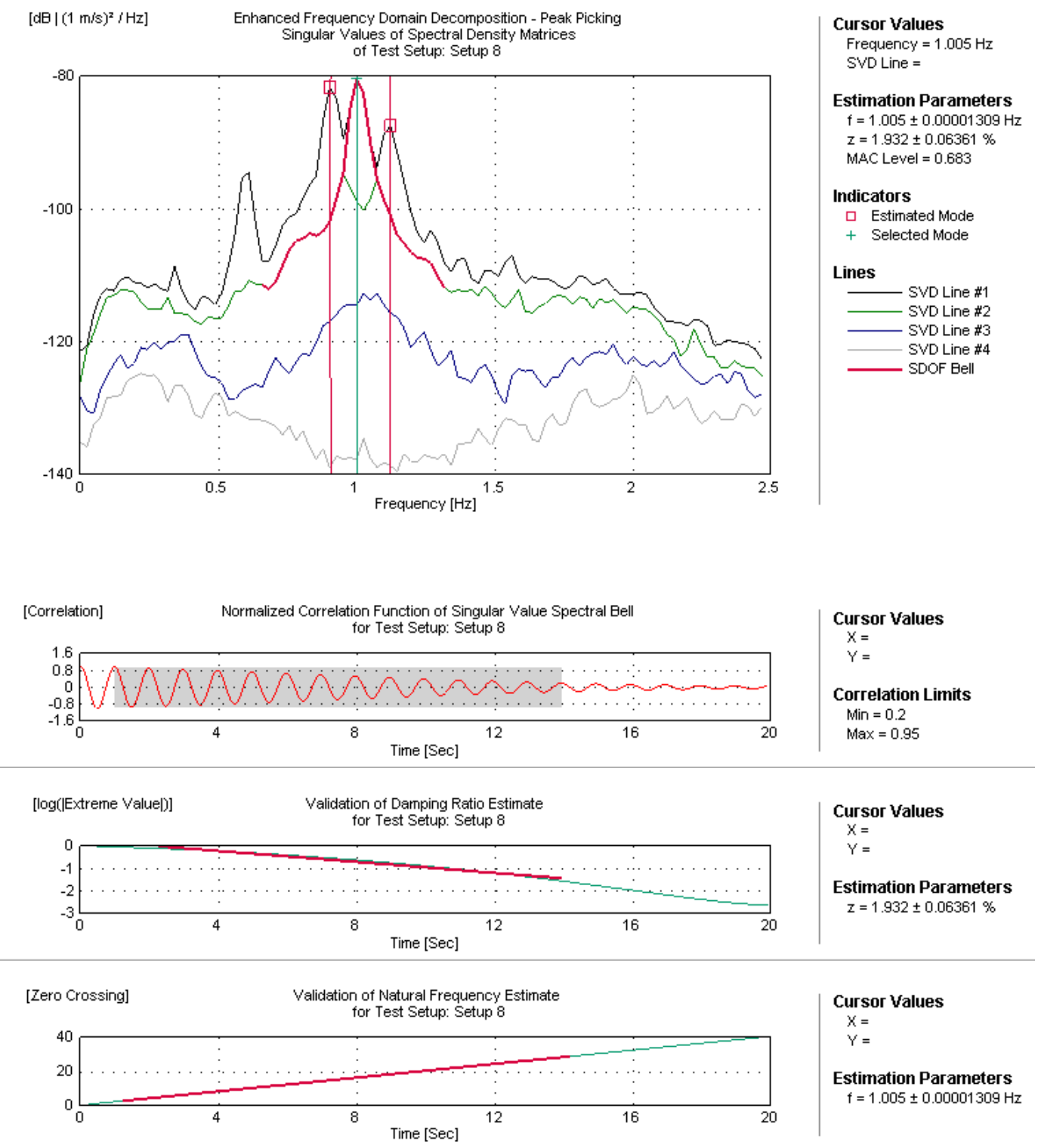


\section{Setup 9:}

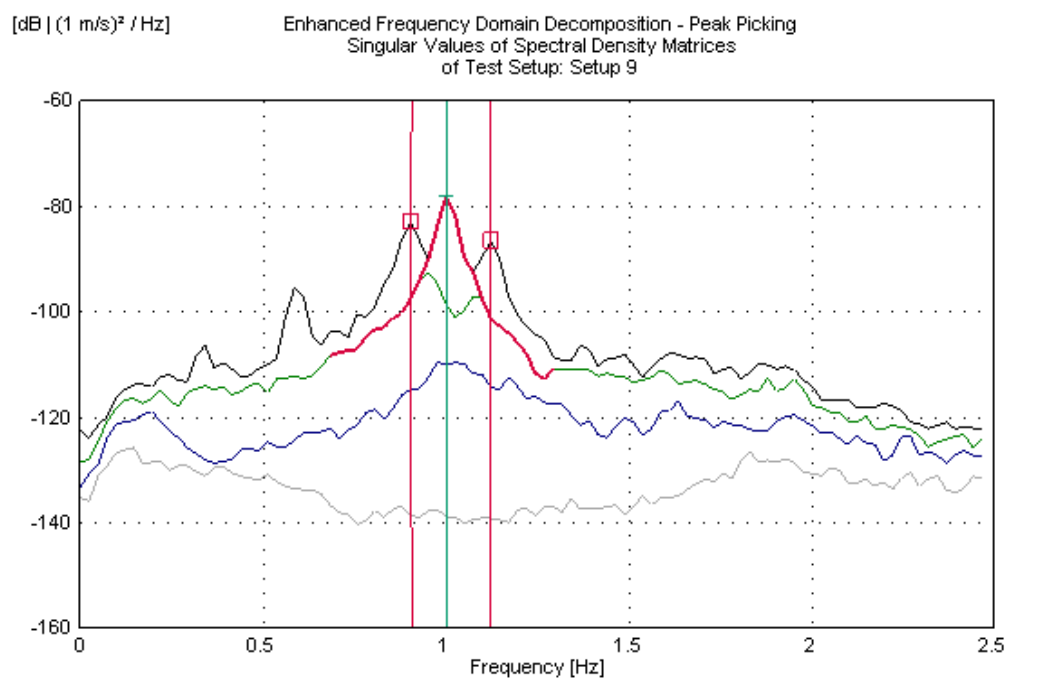

Cursor Values

Frequency $=1.005 \mathrm{~Hz}$ SVD Line =

Estimation Parameters $f=1.003 \pm 0.000006853 \mathrm{~Hz}$

$z=1.511 \pm 0.01078 \%$

MAC Level $=0.6$

Indicators

$\square$ Estimated Mode

+ Selected Mode

Lines

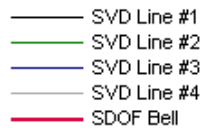

[Correlation] Normalized Correlation Function of Singular Value Spectral Bell for Test Setup: Setup 9

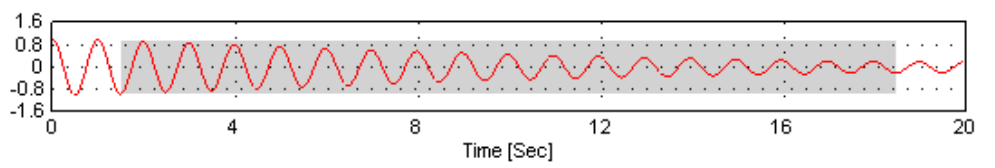

Cursor Values

$X=$

Correlation Limits

$\operatorname{Min}=0.2$

$\operatorname{Max}=0.95$

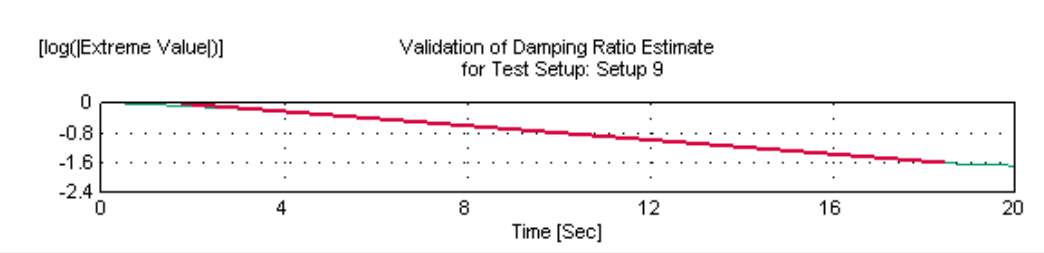

Cursor Values

$X=$

Estimation Parameters

$z=1.511 \pm 0.01078 \%$

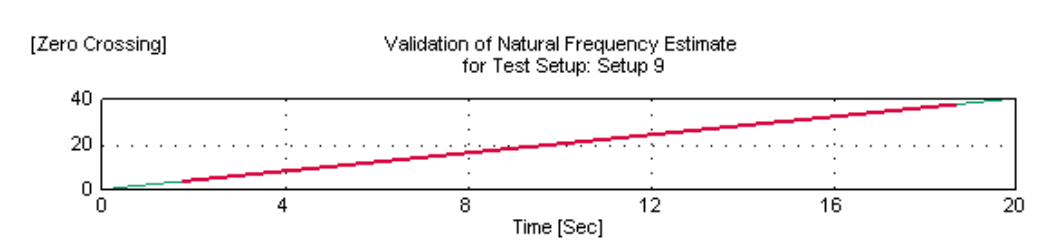

Cursor Values

$X=$
$Y=$

Estimation Parameters $f=1.003 \pm 0.000006853 \mathrm{~Hz}$ 


\section{Setup 10:}
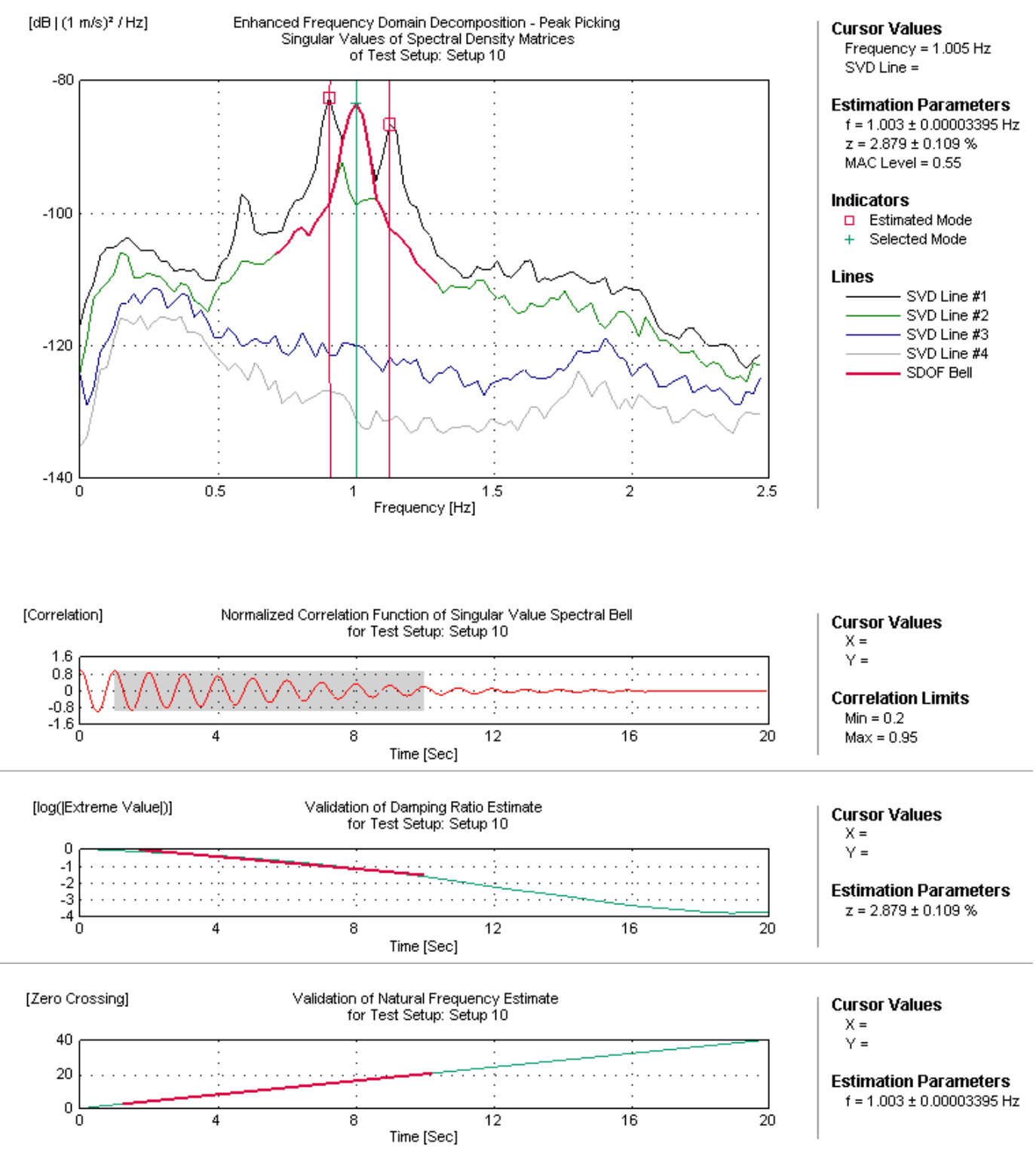


\section{Setup 11:}
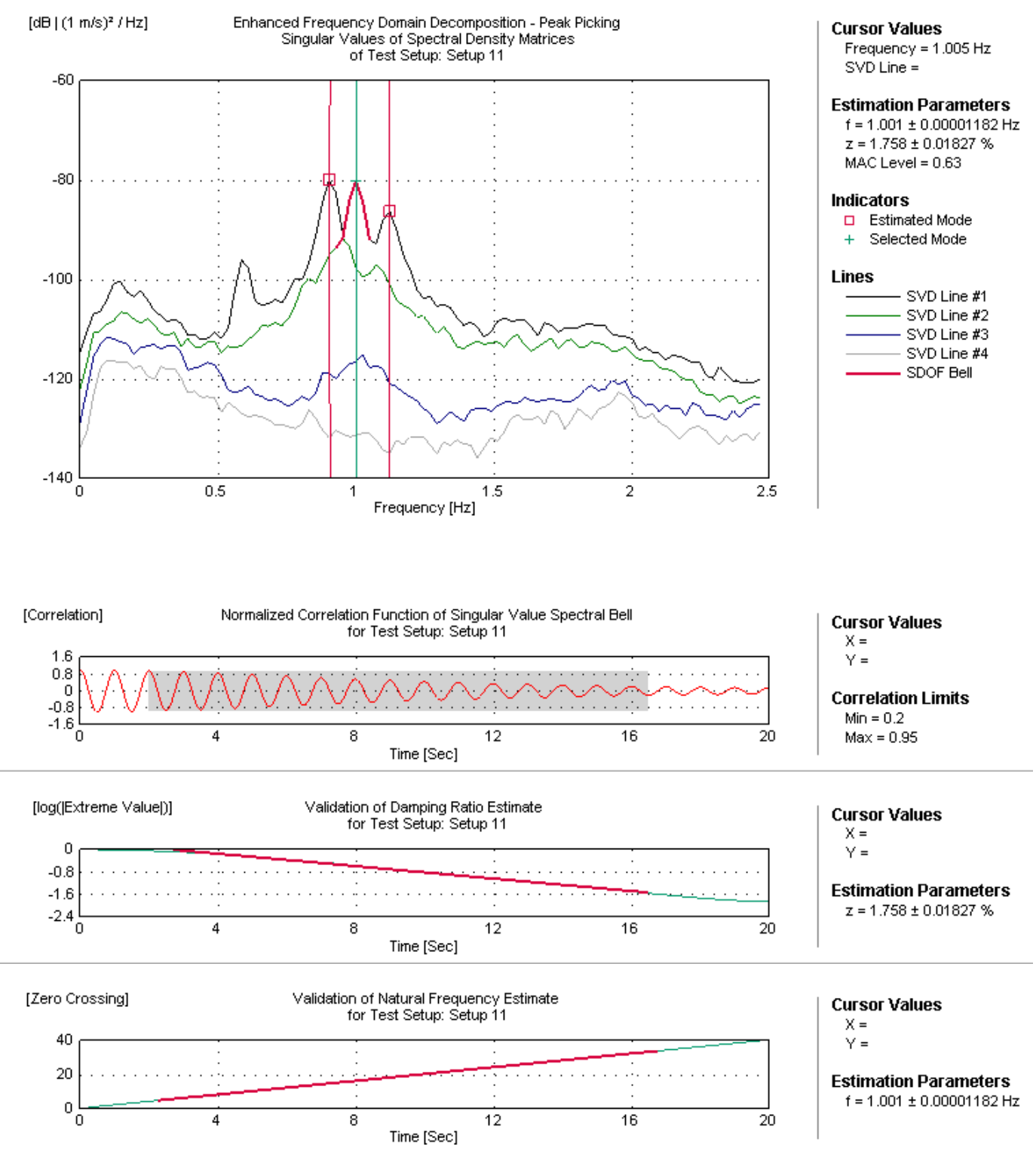


\section{Setup 12:}

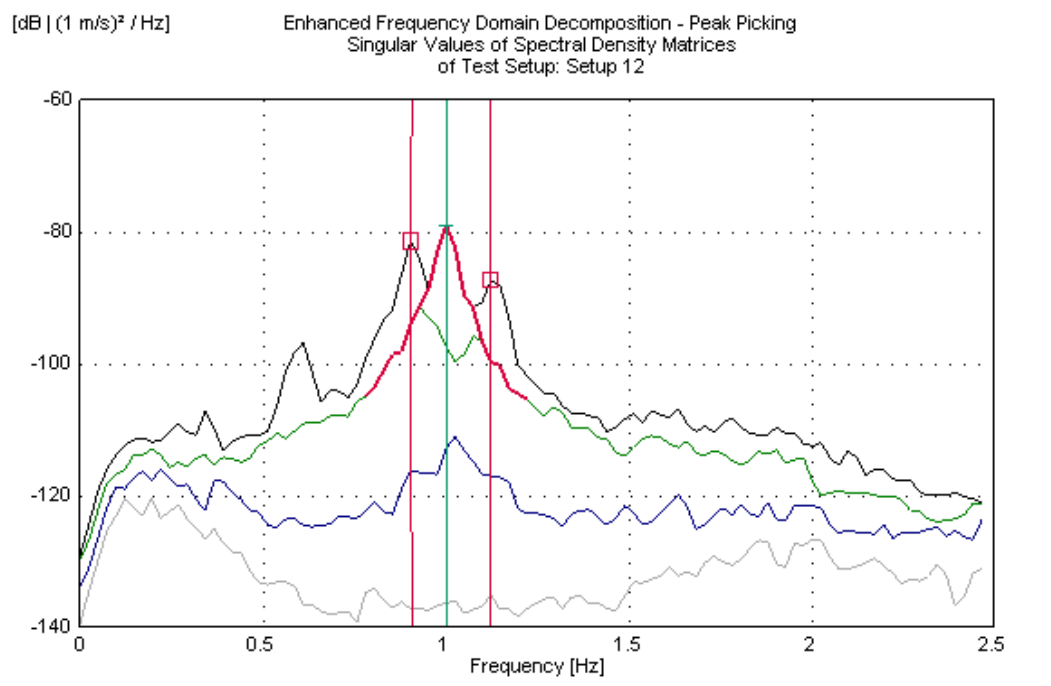

Cursor Values

Frequency $=1.005 \mathrm{~Hz}$ SVD Line =

Estimation Parameters $f=1.003 \pm 0.000002857 \mathrm{~Hz}$

$z=1.787 \pm 0.01441 \%$

MAC Level $=0.7$

Indicators

$\square$ Estimated Mode

+ Selected Mode

Lines

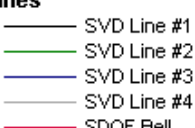

[Correlation] Normalized Correlation Function of Singular Value Spectral Bell for Test Setup: Setup 12

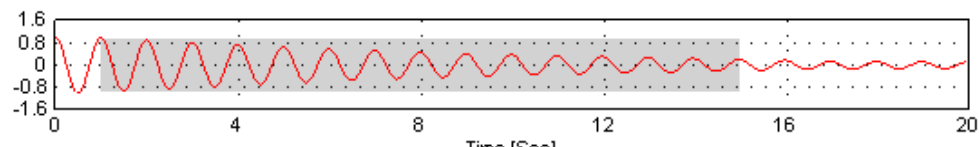

Time [Sec]

[ $\log ($ (Extreme Value|)] Validation of Damping Ratio Estimate

for Test Setup: Setup 12

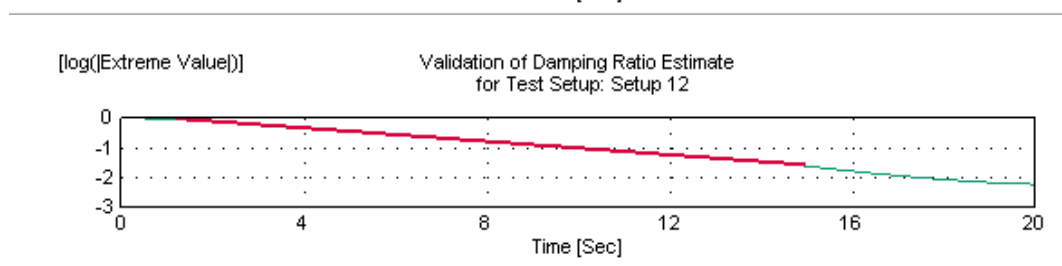

Cursor Values

$X=$

Correlation Limits

$\operatorname{Min}=0.2$

$\operatorname{Max}=0.95$

Cursor Values

$X=$

Estimation Parameters $z=1.787 \pm 0.01441 \%$

[Zero Crossing] Validation of Natural Frequency Estimate

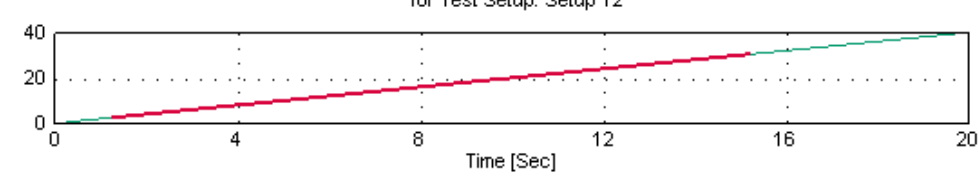

Cursor Values

$X=$
$Y=$

Estimation Parameters $t=1.003 \pm 0.000002857 \mathrm{~Hz}$ 


\section{Setup 13:}
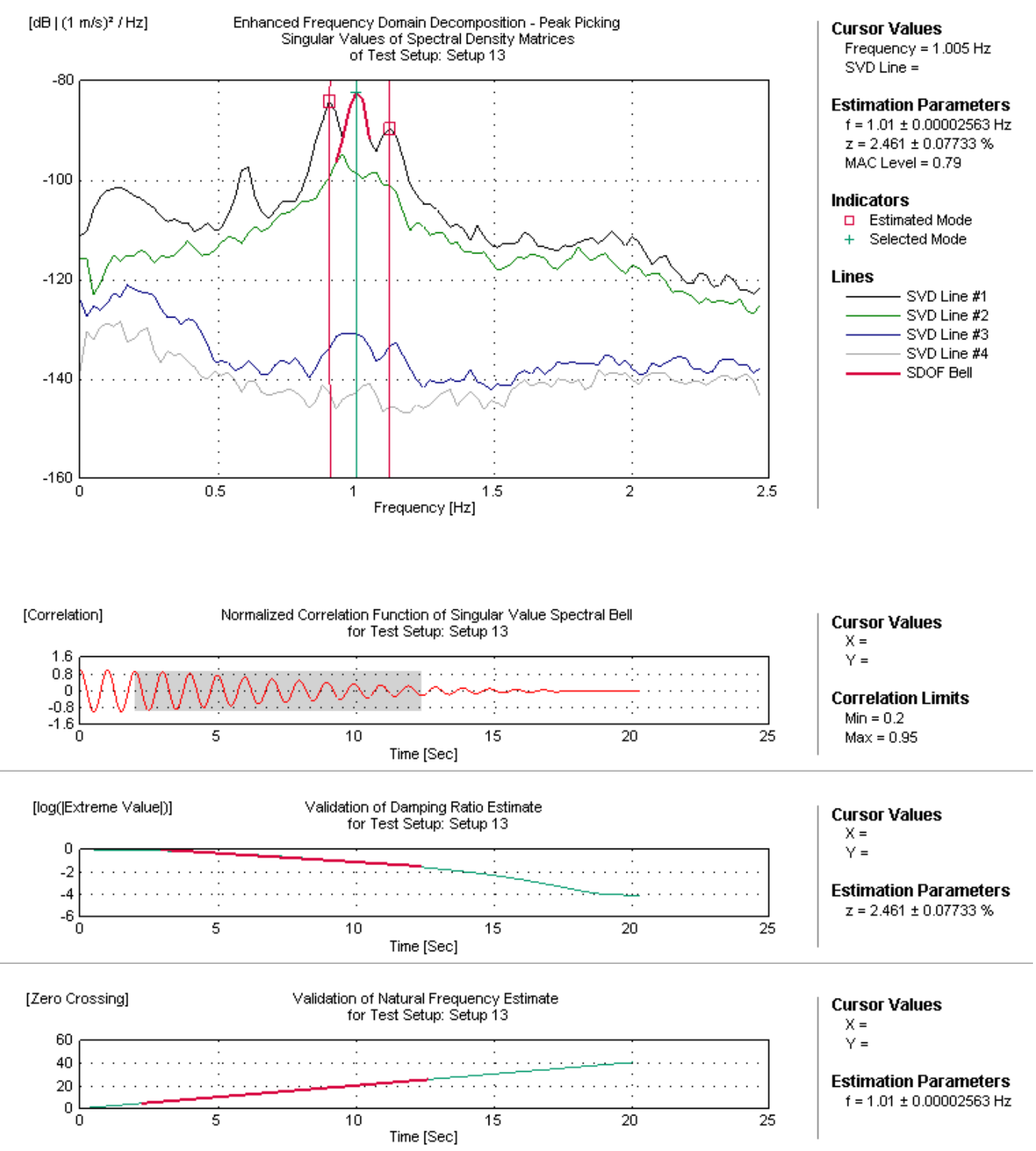


\section{Setup 14:}
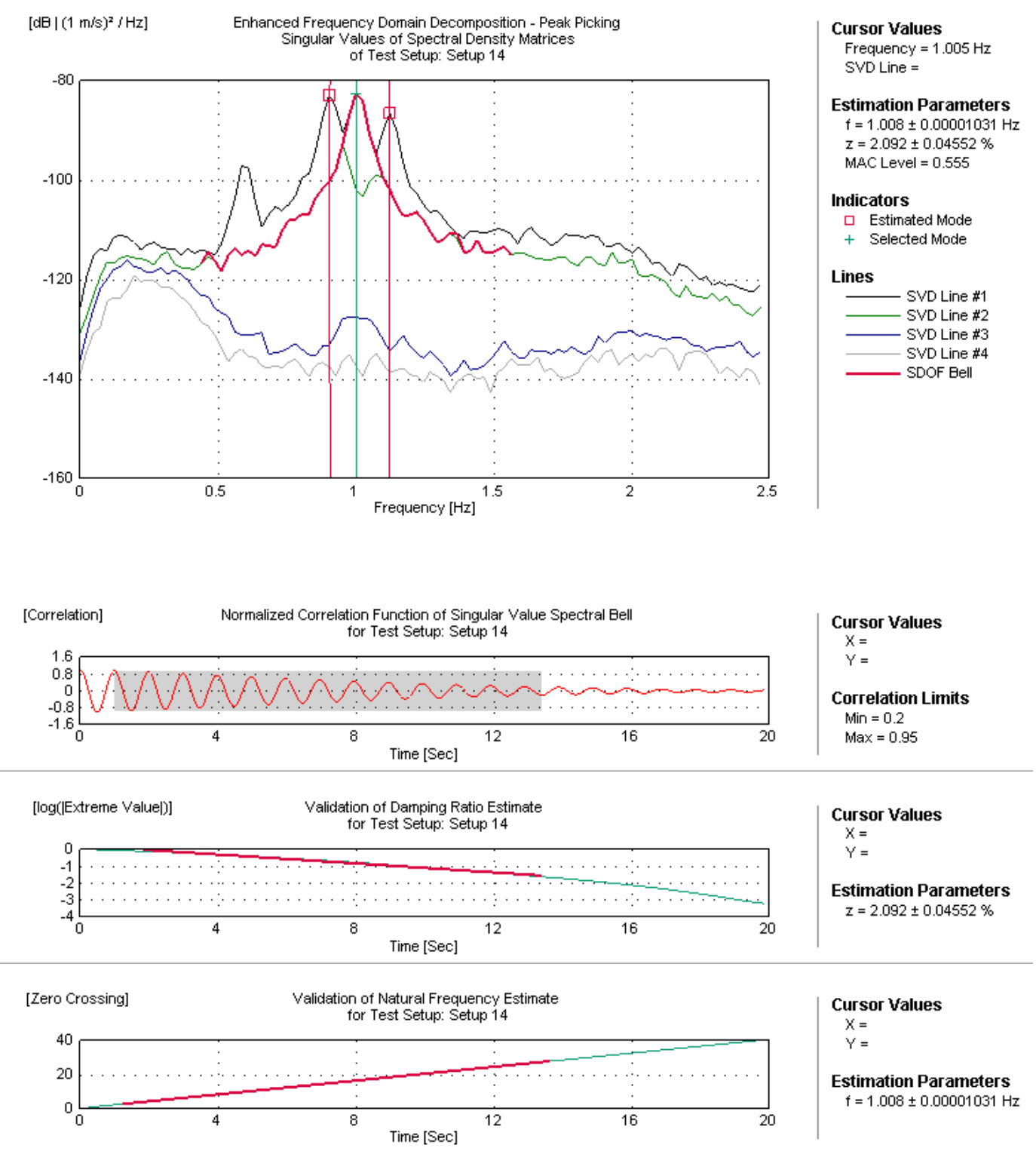


\section{Setup 15:}
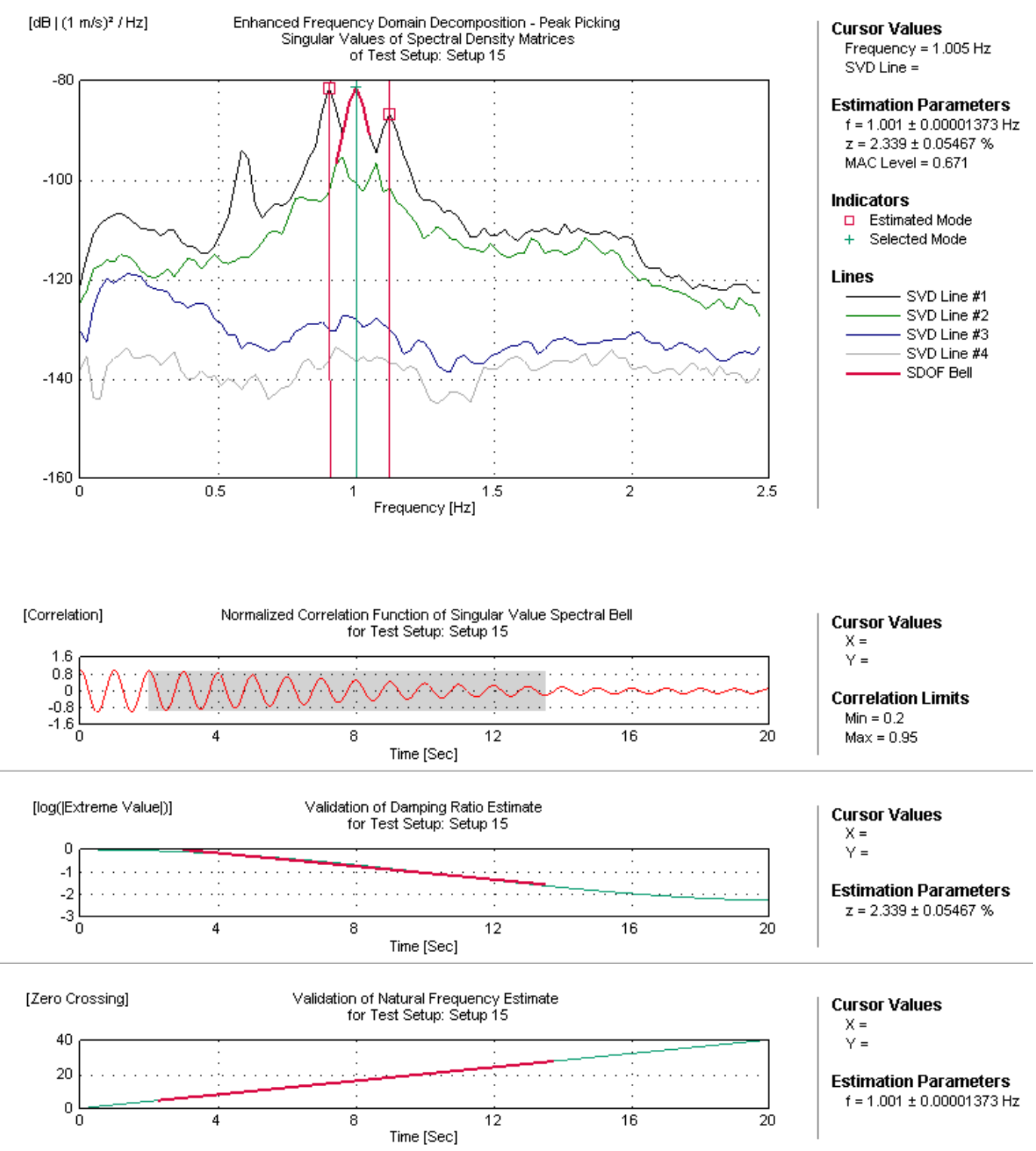


\section{Setup 16:}
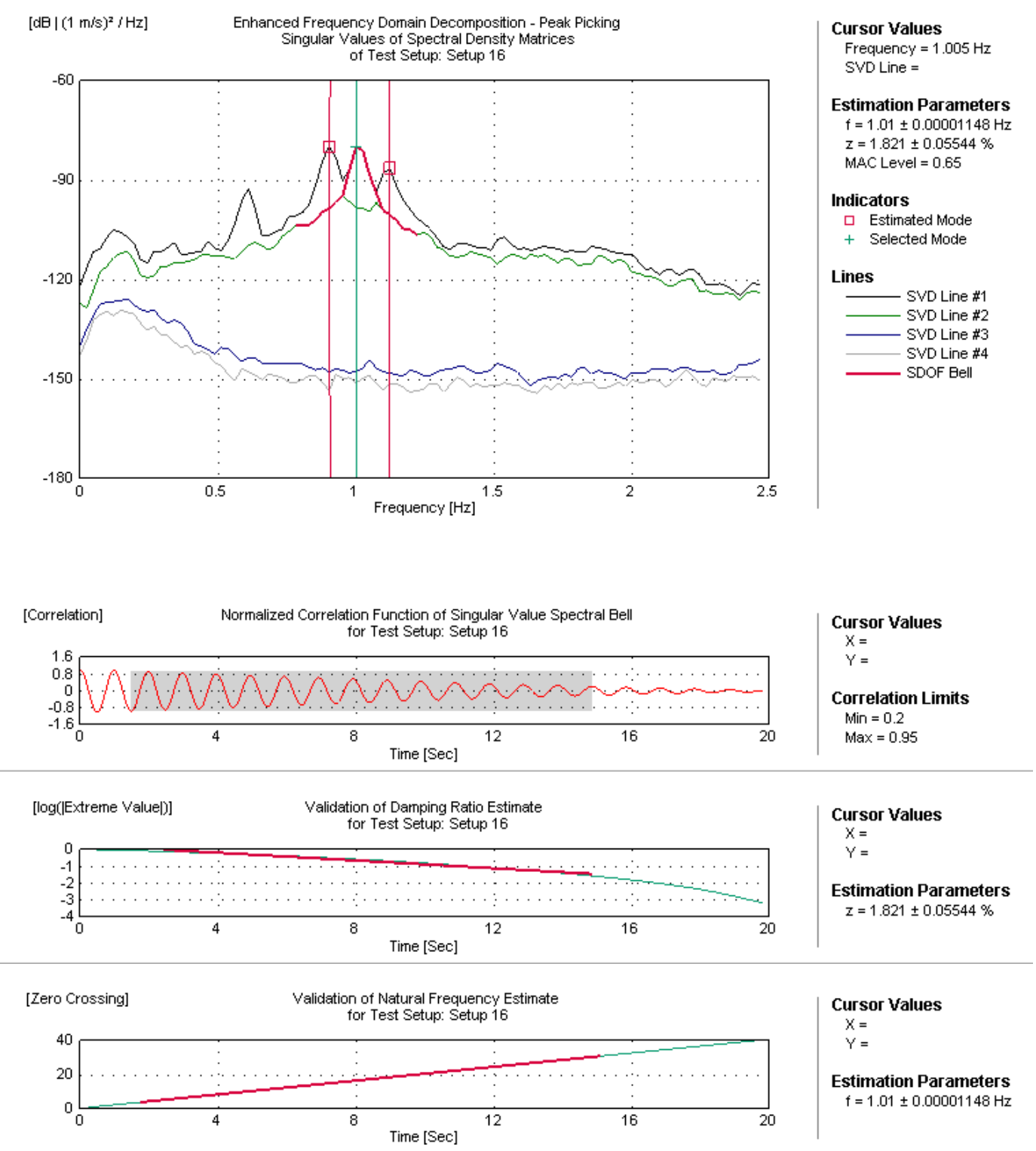


\section{Setup 17:}
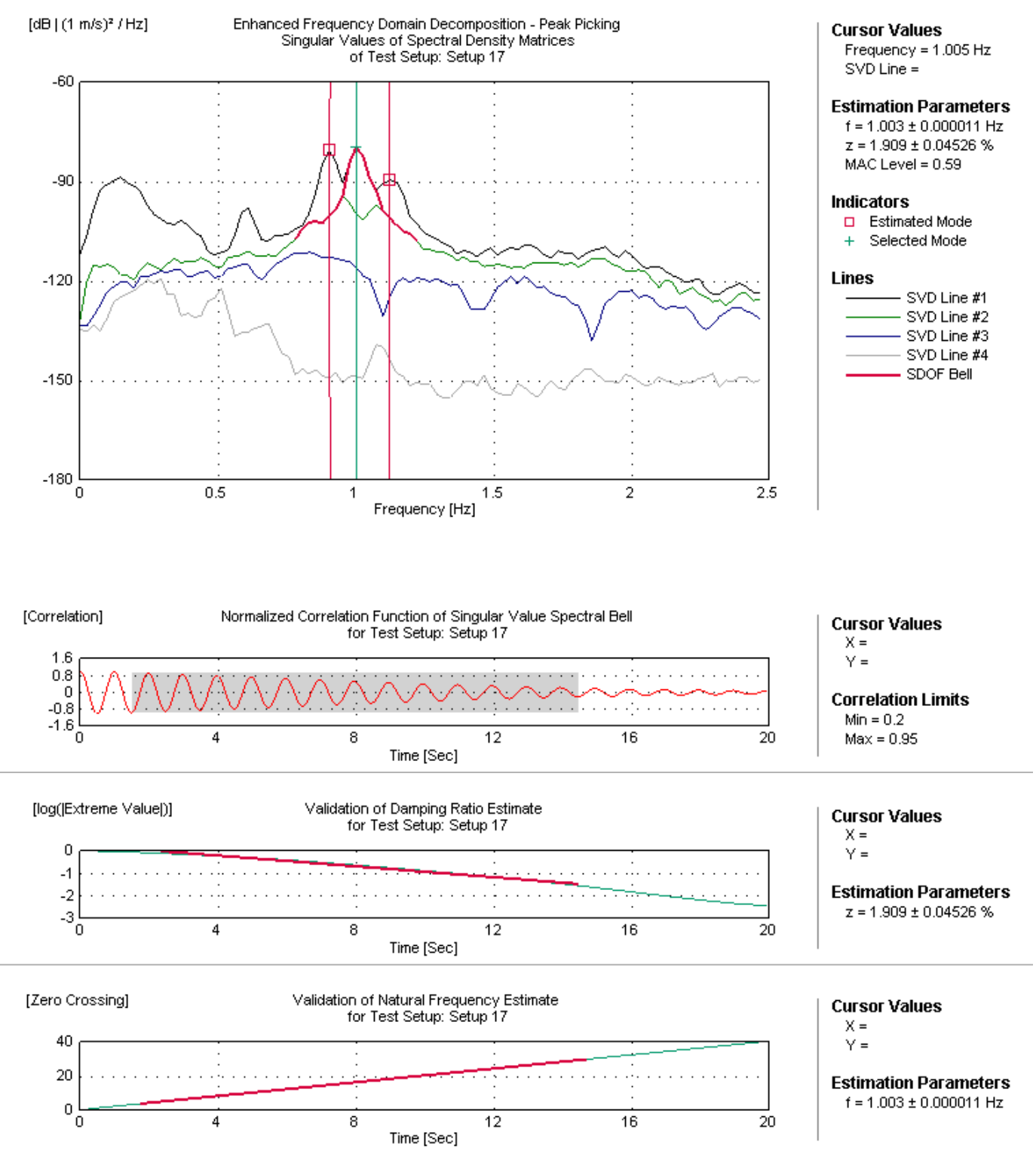


\section{Setup 18:}
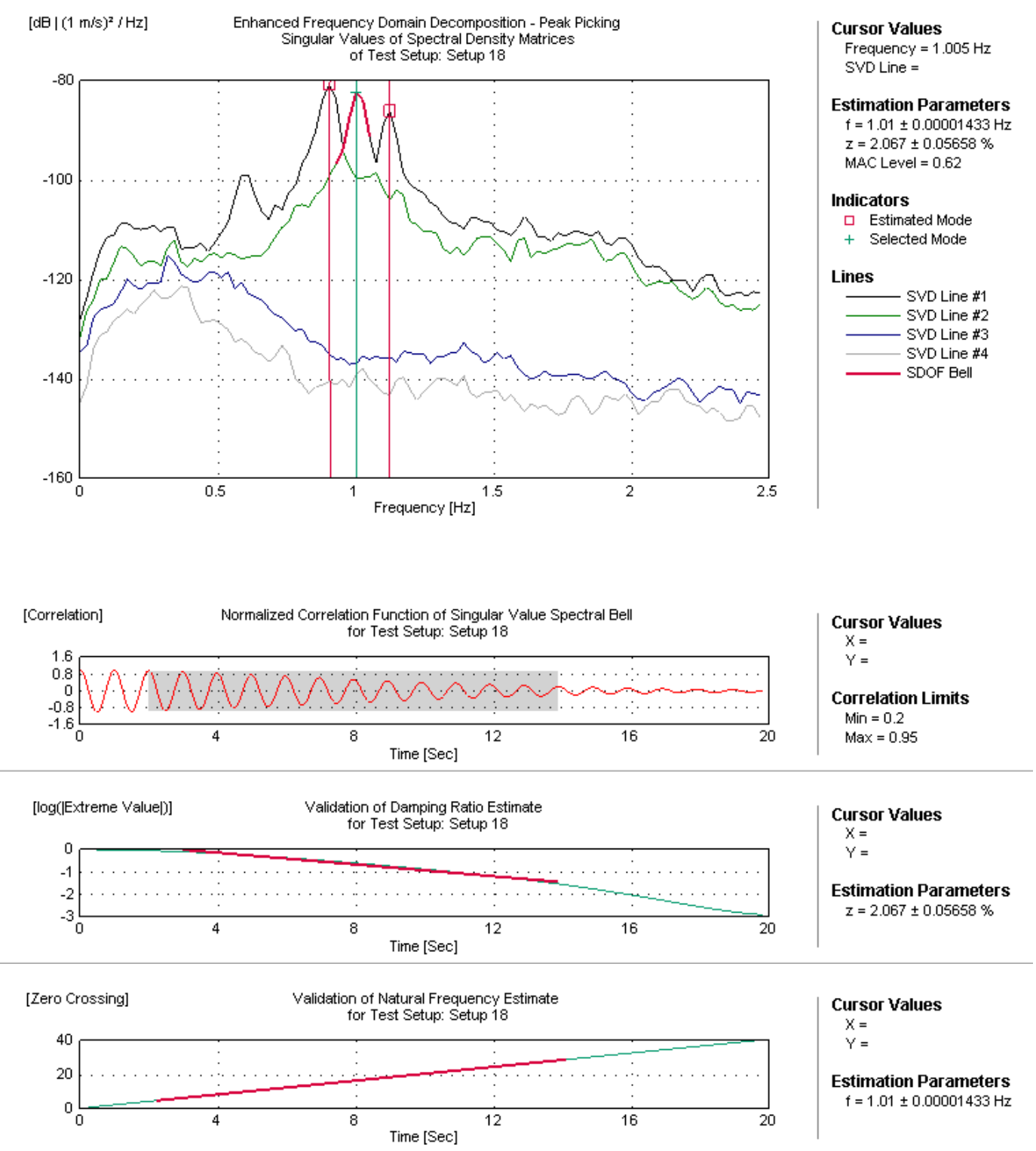


\section{Appendix C:}

\section{Dynamic characteristics of reinforced concrete shear wall buildings in Montréal}


Table C-1: Measured natural frequencies of reinforced concrete shear wall buildings in Montréal

\begin{tabular}{|c|c|c|c|c|c|c|c|c|}
\hline \multirow[b]{3}{*}{ Building } & \multirow{3}{*}{$\begin{array}{c}\text { Number } \\
\text { of } \\
\text { Storeys }\end{array}$} & \multirow{3}{*}{$\begin{array}{l}\text { Height } \\
\text { (m) }\end{array}$} & \multicolumn{6}{|c|}{ Frequency $(\mathrm{Hz})$} \\
\hline & & & \multicolumn{3}{|c|}{ First mode } & \multicolumn{3}{|c|}{ Second mode } \\
\hline & & & Trans & Long & Torsion & Trans & Long & Torsion \\
\hline Hyb-1 & 11 & 38 & RCMRF & 0.77 & 1.04 & RCMRF & 2.41 & NA \\
\hline Hyb-2 & 7 & 25 & 1.52 & 1.58 & 1.90 & 3.72 & 3.92 & $\mathrm{NA}$ \\
\hline Hyb-3 & 8 & 36 & 1.41 & 2.45 & 1.75 & 4.64 & NA & 5.09 \\
\hline Hyb-4 & 47 & 191 & 0.29 & 0.29 & 0.27 & 0.99 & 0.98 & 0.81 \\
\hline RCSW-1 & 15 & 56 & 1.01 & 0.91 & 1.12 & 3.94 & 3.09 & 3.51 \\
\hline RCSW-2 & 49 & 195 & 0.23 & 0.35 & 0.33 & 0.74 & 0.97 & 0.85 \\
\hline RCSW-3 & 28 & 122 & 0.41 & 0.48 & 0.57 & 1.55 & 1.73 & 1.64 \\
\hline RCSW-4 & 27 & 104 & 0.46 & 0.50 & 0.57 & 1.86 & 1.68 & $\mathrm{NA}$ \\
\hline RCSW-5 & 17 & 62 & 0.55 & 0.64 & 0.64 & 2.23 & 2.14 & 1.77 \\
\hline RCSW-6 & 12 & 43 & $1.17^{(\mathrm{C})}$ & $1.30^{(C)}$ & 1.93 & NA & NA & NA \\
\hline RCSW-7 & 6 & 24 & 2.99 & 2.63 & 3.58 & $\mathrm{NA}$ & NA & NA \\
\hline RCSW-8 & 6 & 23 & 2.17 & 2.81 & 1.69 & 6.65 & NA & 5.93 \\
\hline RCSW-9 & 13 & 47 & 1.46 & 1.42 & 2.46 & 4.68 & 4.47 & NA \\
\hline RCSW-10 & 33 & 121 & 0.50 & 0.52 & 0.80 & 1.50 & 1.62 & NA \\
\hline RCSW-11 & 41 & 143 & 0.41 & 0.41 & 0.66 & 1.30 & 1.32 & 1.89 \\
\hline RCSW-12 & 17 & 74 & $0.68^{(\mathrm{C})}$ & $0.76^{(\mathrm{C})}$ & 0.87 & $2.08^{(\mathrm{C})}$ & $1.99^{(\mathrm{C})}$ & 1.63 \\
\hline RCSW-13 & 12 & 45 & 1.01 & 0.89 & 1.40 & $\mathrm{NA}$ & $\mathrm{NA}$ & NA \\
\hline RCSW-14 & 7 & 23 & $3.10^{(C)}$ & $3.14^{(C)}$ & 4.40 & NA & NA & NA \\
\hline RCSW-15 & 16 & 70 & 0.88 & 0.91 & 0.83 & 3.17 & 3.04 & 2.57 \\
\hline RCSW-16 & 6 & 20 & 2.53 & 2.76 & 3.55 & NA & NA & NA \\
\hline RCSW-17 & 22 & 75 & 0.44 & 0.57 & NA & 1.34 & 2.02 & NA \\
\hline RCSW-18 & 6 & 25 & 2.44 & 3.46 & 4.23 & 8.03 & 9.26 & 11.27 \\
\hline RCSW-19 & 15 & 41 & $1.58^{(C)}$ & $1.30^{(C)}$ & 1.93 & $4.74^{(C)}$ & $4.30^{(C)}$ & NA \\
\hline RCSW-20 & 22 & 77 & 0.53 & 0.58 & 0.65 & 1.62 & 2.21 & 1.76 \\
\hline RCSW-21 ${ }^{(a)}$ & 14 & 46 & $1.15^{(\mathrm{c})}$ & $1.04^{(\mathrm{C})}$ & 2.40 & $3.40^{(\mathrm{C})}$ & $3.00^{(\mathrm{C})}$ & NA \\
\hline RCSW-22 (a) & 14 & $50^{(b)}$ & 0.85 & 0.99 & NA & NA & NA & NA \\
\hline RCSW-23 ${ }^{(a)}$ & 11 & 43 & 0.99 & 1.17 & 1.81 & 2.48 & 3.26 & 2.19 \\
\hline
\end{tabular}

(a) Lateral load-resisting system not clearly identified

(b) Value uncertain

(c) Mode shapes skew with respect to building axes of geometry NA: Value could not be identified from ambient vibration records

RCMRF: Reinforced Concrete Moment-Resisting Frame provides resistance to lateral loads 
Table C-2: Measured damping values of reinforced concrete shear wall buildings in Montréal

\begin{tabular}{|c|c|c|c|c|c|c|c|c|}
\hline \multirow[b]{3}{*}{ Building } & \multirow{3}{*}{$\begin{array}{c}\text { Number } \\
\text { of } \\
\text { Storeys }\end{array}$} & \multirow{3}{*}{$\begin{array}{c}\text { Height } \\
(\mathrm{m})\end{array}$} & \multicolumn{6}{|c|}{ Damping (\% critical) } \\
\hline & & & \multicolumn{3}{|c|}{ First mode } & \multicolumn{3}{|c|}{ Second mode } \\
\hline & & & Trans & Long & Torsion & Trans & Long & Torsion \\
\hline Hyb-1 & 11 & 38 & RCMRF & 3.7 & 3.7 & RCMRF & 3.0 & NA \\
\hline Hyb-2 & 7 & 25 & 2.1 & 2.1 & 2.4 & 2.1 & 2.1 & NA \\
\hline Hyb-3 & 8 & 36 & 1.3 & 1.6 & 1.6 & NA & NA & NA \\
\hline Hyb-4 & 47 & 191 & 5.1 & NA & NA & 2.0 & 1.8 & 2.9 \\
\hline RCSW-1 & 15 & 56 & 2.0 & 2.1 & $1.9^{(b)}$ & 1.6 & 1.4 & 1.4 \\
\hline RCSW-2 & 49 & 195 & 2.4 & 1.8 & 2.3 & 0.8 & 1.2 & 1.1 \\
\hline RCSW-3 & 28 & 122 & 3.6 & 3.9 & 3.6 & 1.6 & 1.6 & $1.6^{(\mathrm{b})}$ \\
\hline RCSW-4 & 27 & 104 & 2.3 & 2.2 & 2.7 & 1.4 & 1.2 & NA \\
\hline RCSW-5 & 17 & 62 & 3.4 & 3.2 & NA & 1.3 & 1.7 & 3.0 \\
\hline RCSW-6 & 12 & 43 & $1.9^{(\mathrm{C})}$ & $1.9^{(\mathrm{C})}$ & 1.6 & NA & NA & NA \\
\hline RCSW-7 & 6 & 24 & 3.1 & 4.2 & 3.5 & NA & NA & NA \\
\hline RCSW-8 & 6 & 23 & 3.6 & 1.6 & 3.2 & NA & NA & NA \\
\hline RCSW-9 & 13 & 47 & 1.7 & 1.8 & 2.0 & 2.3 & 2.3 & NA \\
\hline RCSW-10 & 33 & 121 & 2.0 & 1.9 & 1.9 & 1.4 & 1.4 & NA \\
\hline RCSW-11 & 41 & 143 & 3.2 & 3.8 & 3.7 & 1.5 & 1.6 & 1.9 \\
\hline RCSW-12 & 17 & 74 & $2.2^{(\mathrm{c}),(\mathrm{d})}$ & $2.1^{(\mathrm{C}),(\mathrm{d})}$ & $2.3^{(d)}$ & $1.4^{(\mathrm{C}),(\mathrm{d})}$ & $1.3^{(\mathrm{c}),(\mathrm{d})}$ & $1.3^{(\mathrm{d})}$ \\
\hline RCSW-13 & 12 & 45 & 1.9 & 2.3 & 1.9 & NA & NA & NA \\
\hline RCSW-14 & 7 & 23 & $1.1^{(\mathrm{C})}$ & $1.3^{(C)}$ & 0.9 & NA & NA & NA \\
\hline RCSW-15 & 16 & 70 & $1.5^{(\mathrm{d})}$ & $1.2^{(\mathrm{d})}$ & $1.6^{(\mathrm{d})}$ & $1.20^{(\mathrm{d})}$ & $0.90^{(\mathrm{d})}$ & $1.30^{(\mathrm{d})}$ \\
\hline RCSW-16 & 6 & 20 & 2.1 & 1.7 & 2.8 & NA & NA & NA \\
\hline RCSW-17 & 22 & 75 & 4.8 & 3.4 & NA & 2.5 & 2.1 & NA \\
\hline RCSW-18 & 6 & 25 & 1.6 & 1.0 & 1.4 & 2.5 & 1.9 & NA \\
\hline RCSW-19 & 15 & 41 & $1.6^{(C)}$ & $1.7^{(\mathrm{C})}$ & 1.3 & $N A^{(C)}$ & $1.9^{(C)}$ & NA \\
\hline RCSW-20 & 22 & 77 & 3.6 & 3.4 & 3.7 & 1.8 & 2.0 & 2.1 \\
\hline RCSW-21 ${ }^{\text {(a) }}$ & 14 & 46 & $1.8^{(\mathrm{C})}$ & $1.9^{(\mathrm{C})}$ & 1.3 & $1.7^{(\mathrm{C})}$ & $1.9^{(\mathrm{C})}$ & NA \\
\hline RCSW-22 (a) & 14 & $50^{(\mathrm{b})}$ & NA & NA & NA & NA & NA & NA \\
\hline RCSW-23 ${ }^{(a)}$ & 11 & 43 & 2.4 & 2.3 & 1.8 & 2.3 & 2.3 & 2.7 \\
\hline
\end{tabular}

(a) Lateral load-resisting system not clearly identified

(b) Value uncertain

(c) Mode shapes skew with respect to building axes of geometry

(d) Building equipped with supplementary damping devices (not included in results)

NA: Value could not be identified from ambient vibration records

RCMRF: Reinforced Concrete Moment-Resisting Frame provides resistance to lateral loads 


\section{Appendix D:}

\section{Measured fundamental periods of shear wall buildings}


Table D-1: Measured fundamental periods of buildings with shear walls in Montréal

\begin{tabular}{|c|c|c|c|c|c|c|c|c|}
\hline \multirow[b]{2}{*}{ Building ID } & \multicolumn{2}{|c|}{ Type } & \multirow{2}{*}{$\begin{array}{c}\text { Number } \\
\text { of } \\
\text { Storeys }\end{array}$} & \multirow{2}{*}{$\begin{array}{c}\text { Height } \\
(\mathrm{m})\end{array}$} & \multicolumn{2}{|c|}{ Width (m) } & \multicolumn{2}{|c|}{ Period (s) } \\
\hline & Trans & Long & & & Trans & Long & Trans & Long \\
\hline RCSW-1 & USW & CSW & 15 & 56 & 35 & 38 & 0.99 & 1.10 \\
\hline RCSW-2 & USW & CSW & 49 & 195 & $38^{(b)}$ & $82^{(b)}$ & 4.35 & 2.86 \\
\hline RCSW-3 & RCSW & RCSW & 28 & 122 & 37 & 55 & 2.44 & 2.08 \\
\hline RCSW-4 & RCSW & RCSW & 27 & 104 & 38 & 43 & 2.17 & 2.00 \\
\hline RCSW-5 & CusW & CsW & 17 & 62 & 39 & 51 & 1.82 & 1.56 \\
\hline RCSW-6 & CSW & USW & 12 & 43 & $29^{(b)}$ & $30^{(b)}$ & $0.85^{(\mathrm{c})}$ & $0.77^{(\mathrm{c})}$ \\
\hline RCSW-7 & CUSW & USW & 6 & 24 & 27 & 50 & 0.33 & 0.38 \\
\hline RCSW-8 & USW & Cusw & 6 & 23 & 34 & 53 & 0.46 & 0.36 \\
\hline RCSW-9 & CUSW & cusw & 13 & 47 & 30 & 34 & 0.68 & 0.70 \\
\hline RCSW-10 & CSW & csw & 33 & 121 & 47 & 47 & 2.00 & 1.92 \\
\hline RCSW-11 & CSW & CSW & 41 & 143 & 47 & 47 & 2.44 & 2.44 \\
\hline RCSW-12 & Cusw & Cusw & 17 & 74 & $72^{(\mathrm{b})}$ & $89^{(b)}$ & $1.47^{(c)}$ & $1.32^{(c)}$ \\
\hline RCSW-13 & USW & Cusw & 12 & 45 & 37 & 52 & 0.99 & 1.12 \\
\hline RCSW-14 & CUSW & Cusw & 7 & 23 & $21^{(\mathrm{b})}$ & $23^{(b)}$ & $0.32^{(\mathrm{c})}$ & $0.32^{(\mathrm{c})}$ \\
\hline RCSW-15 & RCSW & RCSW & 16 & 70 & 33 & 70 & 1.14 & 1.10 \\
\hline RCSW-16 & USW & USW & 6 & 20 & 25 & 37 & 0.40 & 0.36 \\
\hline RCSW-17 & CSW & USW & 22 & 75 & 46 & 46 & 2.27 & 1.75 \\
\hline RCSW-18 & USW & USW & 6 & 25 & 26 & 31 & 0.41 & 0.29 \\
\hline RCSW-19 & USW & USW & 15 & 41 & $N A^{(b)}$ & $N A^{(b)}$ & $0.63^{(\mathrm{c})}$ & $0.77^{(\mathrm{c})}$ \\
\hline RCSW-20 & $\mathrm{CSW}$ & USW & 22 & 77 & $41^{(\mathrm{b})}$ & $41^{(b)}$ & 1.89 & 1.72 \\
\hline RCSW-21 & RCSW & RCSW & 14 & 46 & 30 & $30^{(\mathrm{b})}$ & $0.87^{(\mathrm{c})}$ & $0.96^{(\mathrm{c})}$ \\
\hline RCSW-22 & RCSW & RCSW & 14 & $50^{(\mathrm{a})}$ & $26^{(a)}$ & $30^{(a)}$ & 1.18 & 1.01 \\
\hline RCSW-23 & RCSW & RCSW & 11 & 43 & 20 & 36 & 1.01 & 0.85 \\
\hline Hyb-1 & RCMRF & Hybrid & 11 & 38 & 34 & 44 & RCMRF & 1.30 \\
\hline Hyb-2 & Hybrid & Hybrid & 7 & 25 & 22 & 36 & 0.66 & 0.63 \\
\hline Hyb-3 & Hybrid & Hybrid & 8 & 36 & 20 & 62 & 0.71 & 0.41 \\
\hline Hyb-4 & Hybrid & Hybrid & 47 & 191 & 43 & 43 & 3.45 & 3.45 \\
\hline
\end{tabular}

(a) Value uncertain

(b) Plan dimensions change along building height and/or building not rectangular

(c) Mode shapes skew with respect to building axes of geometry

NA: $\quad$ Value could not be identified from ambient vibration records

CSW: $\quad$ Coupled shear walls

CUSW: $\quad$ Coupled and uncoupled shear walls

Hyb: Hybrid system involving a combination of shear walls and momentresisting frames

USW: Uncoupled shear walls

RCMRF: Reinforced concrete moment-resisting frames

RCSW: Reinforced concrete shear walls, but no structural plans available 
Table D-2: Measured fundamental periods of shear wall buildings from other studies

\begin{tabular}{|c|c|c|c|c|c|c|c|}
\hline \multirow[b]{2}{*}{ Source } & \multirow[b]{2}{*}{ Building ID } & \multicolumn{2}{|c|}{ Type } & \multirow{2}{*}{$\begin{array}{c}\text { Number } \\
\text { of } \\
\text { Storeys }\end{array}$} & \multirow{2}{*}{$\begin{array}{c}\text { Height } \\
(\mathrm{m})\end{array}$} & \multicolumn{2}{|c|}{ Period (s) } \\
\hline & & Trans & Long & & & Trans & Long \\
\hline$G \& C$ & C58262 & RCSW & RCSW & 2 & 9 & 0.20 & 0.13 \\
\hline$G \& C$ & C24385 & RCSW & RCSW & 10 & 27 & 0.56 & 0.60 \\
\hline$G \& C$ & C24385 & RCSW & RCSW & 10 & 27 & 0.51 & 0.57 \\
\hline$G \& C$ & C58488 & RCSW & RCSW & 4 & 15 & 0.22 & 0.15 \\
\hline$G \& C$ & C14311 & RCSW & RCSW & 5 & 22 & 0.34 & 0.17 \\
\hline$G \& C$ & ATC-3 & RCMRF & RCSW & 12 & 48 & NA & 1.15 \\
\hline$G \& C$ & C24468 & RCSW & RCSW & 8 & 39 & 1.62 & 1.54 \\
\hline$G \& C$ & C24601 & RCSW & RCSW & 17 & 46 & 1.05 & 1.18 \\
\hline$G \& C$ & C24601 & RCSW & RCSW & 17 & 46 & 1.00 & 1.00 \\
\hline$G \& C$ & N253-5 & RCSW & RCSW & 12 & 49 & 1.14 & 1.19 \\
\hline$G \& C$ & N253-5 & RCSW & RCSW & 12 & 49 & 1.13 & 1.07 \\
\hline$G \& C$ & C12284 & RCSW & RCSW & 4 & 15 & 0.60 & 0.50 \\
\hline$G \& C$ & N264-5 & RCSW & RCSW & 10 & 43 & 0.52 & 0.71 \\
\hline$G \& C$ & N264-5 & RCSW & RCSW & 10 & 43 & 0.62 & 0.98 \\
\hline$G \& C$ & N264-5 & RCSW & RCSW & 10 & 43 & 0.62 & 0.97 \\
\hline$G \& C$ & C58334 & RCSW & RCSW & 3 & 11 & 0.18 & 0.18 \\
\hline$G \& C$ & C58348 & RCSW & RCSW & 3 & 12 & 0.46 & 0.38 \\
\hline$G \& C$ & C58394 & RCSW & RCSW & 9 & 32 & 1.30 & 1.20 \\
\hline$G \& C$ & C58394 & RCSW & RCSW & 9 & 32 & 1.45 & 1.00 \\
\hline$G \& C$ & C57355 & RCSW & RCMRF & 10 & 38 & 0.75 & NA \\
\hline$G \& C$ & C57355 & RCSW & RCMRF & 10 & 38 & 0.61 & NA \\
\hline$G \& C$ & C57355 & RCSW & RCMRF & 10 & 38 & 0.61 & NA \\
\hline$G \& C$ & C57356 & RCSW & RCSW & 10 & 29 & 0.43 & 0.73 \\
\hline$G \& C$ & C57356 & RCSW & RCSW & 10 & 29 & 0.42 & 0.70 \\
\hline$G \& C$ & C57356 & RCSW & RCSW & 10 & 29 & 0.43 & 0.65 \\
\hline$G \& C$ & C57356 & RCSW & RCSW & 10 & 29 & 0.41 & 0.63 \\
\hline$G \& C$ & C47459 & RCSW & RCSW & 4 & 20 & 0.35 & 0.24 \\
\hline$F \& B$ & EURO_eur44 & RCSW & RCSW & 8 & 22 & 0.23 & 0.17 \\
\hline$F \& B$ & EURO_eur46 & RCSW & RCSW & 8 & 22 & 0.24 & 0.17 \\
\hline$F \& B$ & EURO_eur48 & RCSW & RCSW & 8 & 22 & 0.22 & 0.17 \\
\hline$F \& B$ & $\mathrm{HOCH} \_b t 1$ & RCSW & RCSW & 5 & 15 & 0.20 & 0.19 \\
\hline$F \& B$ & $\mathrm{HOCH}$ _bt3 & RCSW & RCSW & 5 & 15 & 0.22 & 0.19 \\
\hline$F \& B$ & $\mathrm{HOCH} \_\mathrm{bt} 4$ & RCSW & RCSW & 5 & 15 & 0.18 & 0.17 \\
\hline$F \& B$ & $\mathrm{HOCH} \_\mathrm{bt} 8$ & RCSW & RCSW & 6 & 18 & 0.18 & 0.18 \\
\hline$F \& B$ & $\mathrm{HOCH}$ btA & RCSW & RCSW & 5 & 16 & 0.21 & 0.17 \\
\hline$F \& B$ & $\mathrm{HOCH} \_\mathrm{btB}$ & RCSW & RCSW & 5 & 16 & 0.20 & 0.16 \\
\hline$F \& B$ & $\mathrm{HOCH}$ _btC1 & RCSW & RCSW & 5 & 16 & 0.19 & 0.18 \\
\hline$F \& B$ & $\mathrm{HOCH}+\mathrm{btC2}$ & RCSW & RCSW & 5 & 16 & 0.19 & 0.12 \\
\hline$F \& B$ & $\mathrm{HOCH}$ _btE1 & RCSW & RCSW & 5 & 16 & 0.17 & 0.16 \\
\hline$F \& B$ & $\mathrm{HOCH}$ _btE2 & RCSW & RCSW & 5 & 16 & 0.23 & 0.18 \\
\hline$F \& B$ & SMH_mlg4 & RCSW & RCSW & 9 & 27 & 0.44 & 0.29 \\
\hline$F \& B$ & SMH_mlg5 & RCSW & RCSW & 5 & 16 & 0.24 & 0.16 \\
\hline$F \& B$ & ECHIR_auv4 & RCSW & RCSW & 15 & 43 & 0.61 & 0.60 \\
\hline$F \& B$ & ECHIR_psm3 & RCSW & RCSW & 8 & 22 & 0.43 & 0.37 \\
\hline$F \& B$ & ECHIR_psm4 & RCSW & RCSW & 8 & 22 & 0.45 & 0.36 \\
\hline$F \& B$ & CPUS_condA & RCSW & RCSW & 6 & 17 & 0.35 & 0.52 \\
\hline$F \& B$ & CPUS_arpej & RCSW & RCSW & 16 & 46 & 0.97 & 0.82 \\
\hline$F \& B$ & MIST_bt09 & RCSW & RCSW & 11 & 31 & 0.47 & 0.36 \\
\hline$F \& B$ & MIST_bt10 & RCSW & RCSW & 5 & 16 & 0.17 & 0.12 \\
\hline
\end{tabular}


Table D-2: Measured fundamental periods of shear wall buildings from other studies (... Continued)

\begin{tabular}{|c|c|c|c|c|c|c|c|}
\hline \multirow[b]{2}{*}{ Source } & \multirow[b]{2}{*}{ Building ID } & \multicolumn{2}{|c|}{ Type } & \multirow{2}{*}{$\begin{array}{c}\text { Number } \\
\text { of } \\
\text { Storeys }\end{array}$} & \multirow{2}{*}{$\begin{array}{l}\text { Height } \\
\text { (m) }\end{array}$} & \multicolumn{2}{|c|}{ Period (s) } \\
\hline & & Trans & Long & & & Trans & Long \\
\hline$F \& B$ & MIST_bt11 & RCSW & RCSW & 11 & 31 & 0.45 & 0.36 \\
\hline$F \& B$ & MIST_bt13 & RCSW & RCSW & 9 & 24 & 0.42 & 0.39 \\
\hline$F \& B$ & MIST_bt15 & RCSW & RCSW & 9 & 24 & 0.39 & 0.36 \\
\hline$F \& B$ & MIST_bt25 & RCSW & RCSW & 9 & 25 & 0.39 & 0.37 \\
\hline$F \& B$ & MIST_tr72 & RCSW & RCSW & 18 & 48 & 0.76 & 0.59 \\
\hline$F \& B$ & MIST_tr76 & RCSW & RCSW & 12 & 32 & 0.40 & 0.27 \\
\hline$F \& B$ & VO_aji & RCSW & RCSW & 18 & 49 & 0.78 & 0.63 \\
\hline$F \& B$ & VO_dh32 & RCSW & RCSW & 5 & 13 & 0.21 & 0.17 \\
\hline$F \& B$ & VO_dh34 & RCSW & RCSW & 5 & 13 & 0.17 & 0.21 \\
\hline $\mathrm{K}-\mathrm{C} \& \mathrm{P}$ & S1 & RCSW & RCSW & 73 & 264 & 5.10 & 4.90 \\
\hline $\mathrm{K}-\mathrm{C} \& \mathrm{P}$ & $\mathrm{C} 1$ & RCSW & RCSW & NA & $\mathrm{NA}$ & NA & NA \\
\hline Lee et al & 1 & RCSW & RCSW & 15 & 40 & 0.71 & 1.92 \\
\hline Lee et al & 2 & RCSW & RCSW & 15 & 40 & 1.08 & NA \\
\hline Lee et al & 3 & RCSW & RCSW & 20 & 54 & 1.19 & 1.89 \\
\hline Lee et al & 4 & RCSW & RCSW & 20 & 54 & 1.44 & 1.90 \\
\hline Lee et al & 5 & RCSW & RCSW & 20 & 54 & NA & 1.93 \\
\hline Lee et al & 6 & RCSW & RCSW & 15 & 40 & 1.27 & NA \\
\hline Lee et al & 7 & RCSW & RCSW & 15 & 40 & NA & 2.22 \\
\hline Lee et al & 8 & RCSW & RCSW & 15 & 40 & 1.16 & 1.86 \\
\hline Lee et al & 9 & RCSW & RCSW & 15 & 40 & 1.09 & 1.66 \\
\hline Lee et al & 10 & RCSW & RCSW & 15 & 40 & $\mathrm{NA}$ & 1.93 \\
\hline Lee et al & 11 & RCSW & RCSW & 20 & 54 & $\mathrm{NA}$ & 2.11 \\
\hline Lee et al & 12 & RCSW & RCSW & 15 & 40 & NA & 1.63 \\
\hline Lee et al & 13 & RCSW & RCSW & 15 & 40 & 0.91 & 2.05 \\
\hline Lee et al & 14 & RCSW & RCSW & 20 & 54 & 1.16 & 1.82 \\
\hline Lee et al & 15 & RCSW & RCSW & 20 & 54 & NA & 1.95 \\
\hline Lee et al & 16 & RCSW & RCSW & 20 & 54 & $\mathrm{NA}$ & 1.88 \\
\hline Lee et al & 17 & RCSW & RCSW & 20 & 54 & 1.50 & 1.82 \\
\hline Lee et al & 18 & RCSW & RCSW & 20 & 54 & NA & 1.76 \\
\hline Lee et al & 19 & RCSW & RCSW & 15 & 40 & 0.90 & 1.91 \\
\hline Lee et al & 20 & RCSW & RCSW & 15 & 40 & 0.86 & NA \\
\hline Lee et al & 21 & RCSW & RCSW & 15 & 40 & 1.28 & 1.89 \\
\hline Lee et al & 22 & RCSW & RCSW & 15 & 40 & 0.99 & NA \\
\hline Lee et al & 23 & RCSW & RCSW & 15 & 40 & 1.16 & NA \\
\hline Lee et al & 24 & RCSW & RCSW & 15 & 40 & 1.27 & 1.92 \\
\hline Lee et al & 25 & RCSW & RCSW & 15 & 40 & NA & 1.79 \\
\hline Lee et al & 26 & RCSW & RCSW & 15 & 40 & NA & 1.65 \\
\hline Lee et al & 27 & RCSW & RCSW & 18 & 48 & NA & 1.81 \\
\hline Lee et al & 28 & RCSW & RCSW & 20 & 54 & 1.17 & 1.85 \\
\hline Lee et al & 29 & RCSW & RCSW & 18 & 48 & 1.23 & 1.88 \\
\hline Lee et al & 30 & RCSW & RCSW & 20 & 53 & 1.12 & 1.88 \\
\hline Lee et al & 31 & RCSW & RCSW & 20 & 54 & NA & 1.83 \\
\hline Lee et al & 32 & RCSW & RCSW & 20 & 54 & 1.31 & 1.92 \\
\hline Lee et al & 33 & RCSW & RCSW & 20 & 54 & 1.06 & 1.79 \\
\hline Lee et al & 34 & RCSW & RCSW & 22 & 59 & 1.04 & 1.89 \\
\hline Lee et al & 35 & RCSW & RCSW & 25 & 67 & 1.79 & 2.33 \\
\hline Lee et al & 36 & RCSW & RCSW & 25 & 67 & 1.33 & NA \\
\hline Lee et al & 37 & RCSW & RCSW & 25 & 68 & 1.39 & 2.56 \\
\hline Lee et al & 38 & RCSW & RCSW & 25 & 68 & 1.59 & 2.04 \\
\hline Lee et al & 39 & RCSW & RCSW & 25 & 68 & 1.61 & 2.17 \\
\hline
\end{tabular}


Table D-2: Measured fundamental periods of shear wall buildings from other studies (... Continued)

\begin{tabular}{|c|c|c|c|c|c|c|c|}
\hline \multirow[b]{2}{*}{ Source } & \multirow[b]{2}{*}{ Building ID } & \multicolumn{2}{|c|}{ Type } & \multirow{2}{*}{$\begin{array}{c}\text { Number } \\
\text { of } \\
\text { Storeys }\end{array}$} & \multirow{2}{*}{$\begin{array}{l}\text { Height } \\
\text { (m) }\end{array}$} & \multicolumn{2}{|c|}{ Period (s) } \\
\hline & & Trans & Long & & & Trans & Long \\
\hline Lee et al & 40 & RCSW & RCSW & 25 & 68 & NA & 2.50 \\
\hline Lee et al & 41 & RCSW & RCSW & 25 & 68 & 1.69 & 2.13 \\
\hline Lee et al & 42 & RCSW & RCSW & 19 & 51 & NA & 1.89 \\
\hline Lee et al & 43 & RCSW & RCSW & 20 & 54 & 1.25 & 1.79 \\
\hline Lee et al & 44 & RCSW & RCSW & 15 & 40 & 0.90 & 1.69 \\
\hline Lee et al & 45 & RCSW & RCSW & 20 & 56 & NA & 1.79 \\
\hline Lee et al & 46 & RCSW & RCSW & 20 & 56 & 1.25 & 1.72 \\
\hline Lee et al & 47 & RCSW & RCSW & 20 & 56 & 1.27 & 1.82 \\
\hline Lee et al & 48 & RCSW & RCSW & 20 & 54 & 1.25 & NA \\
\hline Lee et al & 49 & RCSW & RCSW & 20 & 54 & 1.39 & 1.96 \\
\hline Lee et al & 50 & RCSW & RCSW & 20 & 54 & 1.20 & 2.13 \\
\hline$G \& M$ & 1 & Mas SW & Mas SW & 5 & 16 & 0.24 & 0.24 \\
\hline$G \& M$ & 2 & Mas SW & Mas SW & 5 & 16 & 0.27 & 0.25 \\
\hline$G \& M$ & 3 & Mas SW & Mas SW & 5 & 16 & 0.30 & 0.27 \\
\hline$G \& M$ & 4 & Mas SW & Mas SW & 9 & 28 & 0.31 & 0.31 \\
\hline$G \& M$ & 5 & Mas SW & Mas SW & 9 & 28 & 0.41 & 0.41 \\
\hline$G \& M$ & 6 & Mas SW & Mas SW & 9 & 28 & 0.44 & 0.44 \\
\hline$G \& M$ & 7 & Mas SW & Mas SW & 9 & 28 & 0.42 & 0.28 \\
\hline$G \& M$ & 8 & Mas SW & Mas SW & 9 & 28 & 0.41 & 0.52 \\
\hline$G \& M$ & 9 & Prefab SW & Prefab SW & 5 & 15 & 0.20 & 0.22 \\
\hline$G \& M$ & 10 & Prefab SW & Prefab SW & 5 & 15 & 0.22 & 0.23 \\
\hline$G \& M$ & 11 & Prefab SW & Prefab SW & 5 & 15 & 0.23 & 0.24 \\
\hline$G \& M$ & 12 & Prefab SW & Prefab SW & 5 & 17 & 0.21 & 0.20 \\
\hline$G \& M$ & 13 & Prefab SW & Prefab SW & 5 & 17 & 0.24 & 0.21 \\
\hline$G \& M$ & 14 & Prefab SW & Prefab SW & 5 & 17 & 0.30 & 0.24 \\
\hline$G \& M$ & 15 & Prefab SW & Prefab SW & 9 & 30 & 0.31 & 0.29 \\
\hline$G \& M$ & 16 & Prefab SW & Prefab SW & 9 & 30 & 0.38 & 0.35 \\
\hline$G \& M$ & 17 & Prefab SW & Prefab SW & 9 & 30 & 0.42 & 0.39 \\
\hline$G \& M$ & 18 & RCSW & RCSW & 5 & 17 & 0.21 & 0.30 \\
\hline$G \& M$ & 19 & Prefab SW & Prefab SW & 5 & 17 & 0.22 & 0.31 \\
\hline$G \& M$ & 20 & SW & SW & 5 & 17 & 0.23 & 0.33 \\
\hline
\end{tabular}

G \& C: $\quad$ Goel and Chopra (1998)

F \& B: $\quad$ Farsi and Bard (2004)

K-C \& P: Kijewski-Correa and Pirnia (2007)

Lee et al: Lee et al (2000)

G \& M: $\quad$ Ghrib and Mamedov (2004)

Mas SW: Masonry shear walls

Prefab SW: Prefabricated shear walls

RCMRF: Reinforced concrete moment-resisting frames

RCSW: Reinforced concrete shear walls

SW: $\quad$ Shear walls 


\section{Appendix E:}

Statistical analysis on fundamental period data from buildings with different types of shear walls 
To determine whether the data from Montréal buildings having different types of shear walls - uncoupled shear walls (USW), coupled shear walls (CSW), combination of coupled and uncoupled shear walls (CUSW); and hybrid wall-frame systems $(\mathrm{Hyb})$ - can be considered as a single data set, a statistical analysis was performed. First, two separate samples of data were considered: the first sample containing the period data for USW only, and the second containing the period data for all remaining buildings. Since visual inspection of the data (see Figure 5.1) showed a significant correlation between fundamental period and building height, a relationship between these variables was then sought for each sample by performing regression analysis, with a candidate equation of the form

$$
T_{1}=a h^{b}
$$

where $T_{l}$ is the fundamental period in $\mathrm{s}, h$ is the building height in $\mathrm{m}$, and $a$ and $b$ are the parameters to be obtained from regression analysis. To allow the use of standard linear regression techniques, this equation was recast into linear form by taking the natural logarithm of each side:

$$
y=\alpha+b x
$$

where $y=\ln \left(T_{1}\right), x=\ln (h)$, and $\alpha=\ln (a)$.

For each sample, the slope of the regression line was determined using

$$
b=\frac{\left(\sum x_{i} y_{i}\right)-n \bar{x} \bar{y}}{\left(\sum x_{i}^{2}\right)-n \bar{x}^{2}}
$$

where $i$ indicates the $i^{\text {th }}$ data point and the overbar indicates mean value. The intercept of the regression line was then computed from 


$$
\alpha=\bar{y}-b \bar{x}
$$

Finally, the goodness-of-fit of each regression equation was assessed using the standard error of estimate, $s_{e}$, and the coefficient of determination, $R^{2}$.

$$
R^{2}=1-\frac{S S_{e}}{S S_{t}}
$$

where

$$
\begin{aligned}
& S S_{e}=\sum_{i=1}^{n}\left[y_{i}-\left(\alpha+b x_{i}\right)\right]^{2}, \\
& S S_{r}=\sum_{i=1}^{n}\left[\left(\alpha+b x_{i}\right)-\bar{y}\right]^{2}, \text { and } \\
& S S_{t}=S S_{e}+S S_{r}, \\
& S_{e}=\sqrt{\frac{S S_{e}}{n-2}}
\end{aligned}
$$

Figure E-1 shows the results of the regression analyses for both samples, while the data corresponding to each sample are presented in Tables E-1 and E-2. Notice that there is a difference between the regression lines obtained for the two samples. To determine whether this difference was statistically significant, a series of hypothesis tests was performed, using the methods described in Zar (1999). 


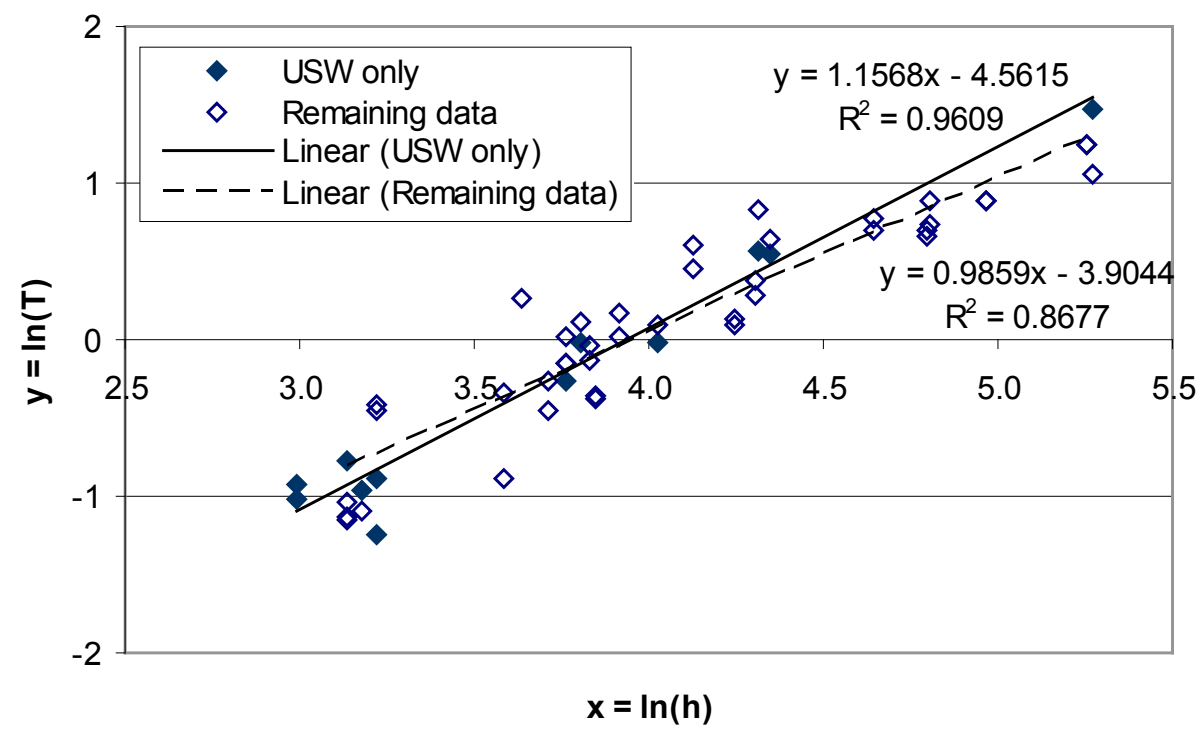

Figure E-1: Results of regression analyses for two samples of period data

\section{Test on equality of variances}

First, the hypothesis that the variance of the two samples could be considered equal was tested using the $F$-distribution.

Null hypothesis $\left(H_{0}\right): \quad \frac{\sigma_{1}^{2}}{\sigma_{2}^{2}}=1$

Alternative hypothesis $\left(H_{1}\right): \quad \frac{\sigma_{1}^{2}}{\sigma_{2}{ }^{2}} \neq 1$

Sample 1:

$$
\begin{aligned}
& \alpha_{1}=-4.5615 \\
& \beta_{1}=1.1568 \\
& n_{1}=12 \\
& \bar{x}_{1}=3.6892 \\
& \bar{y}_{1}=-0.2937
\end{aligned}
$$

Sample 2:

$$
\begin{aligned}
& \alpha_{2}=-3.9044 \\
& \beta_{2}=0.9859 \\
& n_{2}=41 \\
& \bar{x}_{2}=4.0918 \\
& \bar{y}_{2}=0.1299
\end{aligned}
$$




$$
\begin{array}{ll}
S_{e 1}=0.1721 & S_{e 2}=0.2413 \\
S S_{e 1}=0.2961 & S S_{e 2}=2.2709 \\
\sum\left(x_{i}-\bar{x}\right)_{1}^{2}=5.4357 & \sum\left(x_{i}-\bar{x}\right)_{2}^{2}=15.3238 \\
\sum\left(x_{i}-\bar{x}\right)\left(y_{i}-\bar{y}\right)_{1}=6.2883 & \sum\left(x_{i}-\bar{x}\right)\left(y_{i}-\bar{y}\right)_{2}=15.1084 \\
\sum\left(y_{i}-\bar{y}\right)_{1}^{2}=7.5707 & \sum\left(y_{i}-\bar{y}\right)_{2}^{2}=17.1669
\end{array}
$$

Since $s_{e 2}$ is larger than $s_{e l}, F$ was computed as

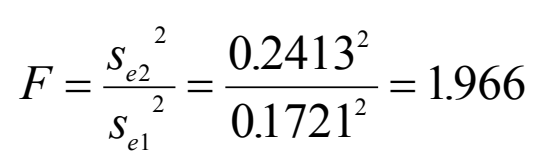

The numerator degrees of freedom (sample 2) and the denominator degrees of freedom (sample 1) are

$$
\begin{aligned}
& v_{\text {num }}=n_{2}-1=40 \\
& v_{\text {den }}=n_{1}-1=11
\end{aligned}
$$

The value of the F-distribution corresponding to a probability of 0.025 (one-tailed test), and the above numerator and denominator degrees of freedom is approximately 3.06 . Since $F=1.966<3.06$, the null hypothesis was not rejected, and the difference between the variances of samples 1 and 2 was not deemed to be statistically significant.

\section{Test on equality of slopes of regression lines}

Next, the hypothesis that the slopes of the regression lines of both samples could be considered equal was tested using the $t$-test. 
Null hypothesis $\left(H_{0}\right): \quad \beta_{1}-\beta_{2}=0$

Alternative hypothesis $\left(H_{1}\right): \quad \beta_{1}-\beta_{2} \neq 0$

Since the difference between the variances of the two samples was not statistically significant, the pooled residual mean square (or pooled standard error of estimate squared) was calculated as

$$
S_{p}{ }^{2}=\frac{S S_{e 1}+S S_{e 2}}{n_{1}+n_{2}-4}=\frac{0.2961+2.2709}{12+41-4}=0.0524
$$

The standard error of the difference between the slopes was then calculated using

$$
S_{\beta_{1}-\beta_{2}}=\sqrt{\frac{s_{p}{ }^{2}}{\sum\left(x_{i}-\bar{x}\right)^{2}{ }_{1}}+\frac{s_{p}{ }^{2}}{\sum\left(x_{i}-\bar{x}\right)^{2}{ }_{2}}}=\sqrt{\frac{0.0524}{5.4357}+\frac{0.0524}{15.3238}}=0.1143
$$

Then,

$$
t=\left|\frac{\beta_{1}-\beta_{2}}{s_{\beta_{1}-\beta_{2}}}\right|=\left|\frac{1.1568-0.9859}{0.1143}\right|=1.496
$$

And the number of degrees of freedom is

$$
v=n_{1}+n_{2}-4=12+41-4=49
$$

The value of the $t$-distribution corresponding to a probability of 0.025 (onetailed test), and 49 degrees of freedom is 2.010 . 
Since $t=1.496<2.010$, the null hypothesis was not rejected, and the difference between the slopes of the regression lines for the two samples was not deemed to be statistically significant.

\section{Test on equality of intercepts of regression lines}

Finally, the hypothesis that the intercepts of the regression lines of both samples could be considered equal was tested using the $t$-test.

Null hypothesis $\left(H_{0}\right): \quad \alpha_{1}-\alpha_{2}=0$

Alternative hypothesis $\left(H_{1}\right): \quad \alpha_{1}-\alpha_{2} \neq 0$

The sums of squares for the "common regression" (i.e. the regression obtained considering all data) were calculated as follows

$$
\begin{aligned}
& A_{c}=\sum\left(x_{i}-\bar{x}\right)^{2}{ }_{1}+\sum\left(x_{i}-\bar{x}\right)^{2}{ }_{2}=5.4357+15.3238=20.7595 \\
& B_{c}=\sum\left(x_{i}-\bar{x}\right)\left(y_{i}-\bar{y}\right)_{1}+\sum\left(x_{i}-\bar{x}\right)\left(y_{i}-\bar{y}\right)_{2}=6.2883+15.1084=21.3967 \\
& C_{c}=\sum\left(y_{i}-\bar{y}\right)^{2}{ }_{1}+\sum\left(y_{i}-\bar{y}\right)^{2}{ }_{2}=7.5707+17.1669=24.7375 \\
& S S_{c}=C_{c}-\frac{B_{c}{ }^{2}}{A_{c}}=24.7375-\frac{21.3967^{2}}{20.7595}=2.6842
\end{aligned}
$$

The slope of the "common regression" line was then obtained using

$$
b_{c}=\frac{B_{c}}{A_{c}}=\frac{21.3967}{20.7595}=1.0307
$$

The number of residual degrees of freedom for the "common regression" is 


$$
v_{c}=n_{1}+n_{2}-3=12+41-3=50
$$

And the residual mean square for the "common regression" was computed from

$$
\left(s_{e}^{2}\right)_{c}=\frac{S S_{c}}{v_{c}}=\frac{2.6842}{50}=0.0537
$$

Then,

$$
t=\left|\frac{\left(\bar{y}_{1}-\bar{y}_{2}\right)-b_{c}\left(\bar{x}_{1}-\bar{x}_{2}\right)}{\sqrt{\left(s_{e}{ }^{2}\right)_{c}\left[\frac{1}{n_{1}}+\frac{1}{n_{2}}+\frac{\left(\bar{x}_{1}-\bar{x}_{2}\right)^{2}}{A_{c}}\right]}}\right|=0.1096
$$

The value of the $t$-distribution corresponding to a probability of 0.025 (onetailed test), and 50 degrees of freedom is 2.009 .

Since $t=0.1096<2.009$, the null hypothesis was not rejected, and the difference between the intercepts of the regression lines for the two samples was not deemed to be statistically significant.

From the above hypothesis tests, it was concluded that the difference between the regression lines corresponding to the two samples (USW and all other buildings with shear walls) was not statistically significant. It was thus concluded that the period data from the 27 buildings could be considered as a single data set. 
Table E-1: Data for sample 1 (USW)

\begin{tabular}{|c|c|c|c|c|c|}
\hline Direction & Building & $\mathbf{h}_{\mathbf{i}}(\mathbf{m})$ & $\mathbf{T}_{\mathbf{i}}(\mathbf{s})$ & $\mathbf{x}_{\mathbf{i}}=\ln \left(\mathbf{h}_{\mathbf{i}}\right)$ & $\mathbf{y}_{\mathbf{i}}=\operatorname{In}\left(\mathbf{T}_{\mathbf{i}}\right)$ \\
\hline \multirow{4}{*}{ Transverse } & RCSW-1 & 56 & 0.99 & 4.0254 & -0.0100 \\
\cline { 2 - 6 } & RCSW-2 & 195 & 4.35 & 5.2730 & 1.4697 \\
\cline { 2 - 6 } & RCSW-8 & 23 & 0.46 & 3.1355 & -0.7747 \\
\cline { 2 - 6 } & RCSW-13 & 45 & 0.99 & 3.8067 & -0.0100 \\
\cline { 2 - 6 } & RCSW-16 & 20 & 0.40 & 2.9957 & -0.9282 \\
\cline { 2 - 6 } & RCSW-18 & 25 & 0.41 & 3.2189 & -0.8920 \\
\hline \multirow{4}{*}{ Long } & RCSW-6 & 43 & 0.77 & 3.7612 & -0.2624 \\
\cline { 2 - 6 } & RCSW-7 & 24 & 0.38 & 3.1781 & -0.9670 \\
\cline { 2 - 6 } & RCSW-16 & 20 & 0.36 & 2.9957 & -1.0152 \\
\cline { 2 - 6 } & RCSW-17 & 75 & 1.75 & 4.3175 & 0.5621 \\
\cline { 2 - 6 } & RCSW-18 & 25 & 0.29 & 3.2189 & -1.2413 \\
\cline { 2 - 6 } & RCSW-20 & 77 & 1.72 & 4.3438 & 0.5447 \\
\hline
\end{tabular}

Table E-2: Data for sample 2 (All remaining data)

\begin{tabular}{|c|c|c|c|c|c|}
\hline Direction & Building & $h_{i}(m)$ & $T_{i}(s)$ & $x_{i}=\ln \left(h_{i}\right)$ & $y_{i}=\ln \left(T_{i}\right)$ \\
\hline \multirow{20}{*}{ Transverse } & Hyb-2 & 25 & 0.66 & 3.2189 & -0.4187 \\
\hline & Hyb-3 & 36 & 0.71 & 3.5835 & -0.3436 \\
\hline & Hyb-4 & 191 & 3.45 & 5.2523 & 1.2379 \\
\hline & RCSW-3 & 122 & 2.44 & 4.8040 & 0.8916 \\
\hline & RCSW-4 & 104 & 2.17 & 4.6444 & 0.7765 \\
\hline & RCSW-5 & 62 & 1.82 & 4.1271 & 0.5978 \\
\hline & RCSW-6 & 43 & 0.85 & 3.7612 & -0.1570 \\
\hline & RCSW-7 & 24 & 0.33 & 3.1781 & -1.0953 \\
\hline & RCSW-9 & 47 & 0.68 & 3.8501 & -0.3784 \\
\hline & RCSW-10 & 121 & 2.00 & 4.7958 & 0.6931 \\
\hline & RCSW-11 & 143 & 2.44 & 4.9628 & 0.8916 \\
\hline & RCSW-12 & 74 & 1.47 & 4.3041 & 0.3857 \\
\hline & RCSW-14 & 23 & 0.32 & 3.1355 & -1.1314 \\
\hline & RCSW-15 & 70 & 1.14 & 4.2485 & 0.1278 \\
\hline & RCSW-17 & 75 & 2.27 & 4.3175 & 0.8210 \\
\hline & RCSW-19 & 41 & 0.63 & 3.7136 & -0.4574 \\
\hline & RCSW-20 & 77 & 1.89 & 4.3438 & 0.6349 \\
\hline & RCSW-21 & 46 & 0.87 & 3.8286 & -0.1398 \\
\hline & RCSW-22 & 50 & 1.18 & 3.9120 & 0.1625 \\
\hline & RCSW-23 & 43 & 1.01 & 3.7612 & 0.0101 \\
\hline \multirow{16}{*}{ Long } & Hyb-1 & 38 & 1.30 & 3.6376 & 0.2614 \\
\hline & Hyb-2 & 25 & 0.63 & 3.2189 & -0.4574 \\
\hline & Hyb-3 & 36 & 0.41 & 3.5835 & -0.8961 \\
\hline & Hyb-4 & 191 & 3.45 & 5.2523 & 1.2379 \\
\hline & RCSW-1 & 56 & 1.10 & 4.0254 & 0.0943 \\
\hline & RCSW-2 & 195 & 2.86 & 5.2730 & 1.0498 \\
\hline & RCSW-3 & 122 & 2.08 & 4.8040 & 0.7340 \\
\hline & RCSW-4 & 104 & 2.00 & 4.6444 & 0.6931 \\
\hline & RCSW-5 & 62 & 1.56 & 4.1271 & 0.4463 \\
\hline & RCSW-8 & 23 & 0.36 & 3.1355 & -1.0332 \\
\hline & RCSW-9 & 47 & 0.70 & 3.8501 & -0.3507 \\
\hline & RCSW-10 & 121 & 1.92 & 4.7958 & 0.6539 \\
\hline & RCSW-11 & 143 & 2.44 & 4.9628 & 0.8916 \\
\hline & RCSW-12 & 74 & 1.32 & 4.3041 & 0.2744 \\
\hline & RCSW-13 & 45 & 1.12 & 3.8067 & 0.1165 \\
\hline & RCSW-14 & 23 & 0.32 & 3.1355 & -1.1442 \\
\hline
\end{tabular}


Table E-2: Data for sample 2 (All remaining data) (... Continued)

\begin{tabular}{|c|c|c|c|c|c|}
\hline Direction & Building & $\mathbf{h}_{\mathbf{i}}(\mathbf{m})$ & $\mathbf{T}_{\mathbf{i}}(\mathbf{s})$ & $\mathbf{x}_{\mathbf{i}}=\ln \left(\mathbf{h}_{\mathbf{i}}\right)$ & $\mathbf{y}_{\mathbf{i}}=\mathbf{I}\left(\mathbf{T}_{\mathbf{i}}\right)$ \\
\hline \multirow{4}{*}{ Long } & RCSW-15 & 70 & 1.10 & 4.2485 & 0.0943 \\
\cline { 2 - 6 } & RCSW-19 & 41 & 0.77 & 3.7136 & -0.2624 \\
\cline { 2 - 6 } & RCSW-21 & 46 & 0.96 & 3.8286 & -0.0392 \\
\cline { 2 - 6 } & RCSW-22 & 50 & 1.01 & 3.9120 & 0.0101 \\
\cline { 2 - 6 } & RCSW-23 & 43 & 0.85 & 3.7612 & -0.1570 \\
\hline
\end{tabular}




\section{Appendix F:}

Wall dimensions of selected buildings in Montréal 


\section{Building ID: RCSW-1}

Storeys: 15

Height: $56 \mathrm{~m}$

Area: $1310 \mathrm{~m}^{2}$

\section{Transverse direction}

Uncoupled shear walls

\begin{tabular}{|c|c|c|c|c|c|c|c|c|c|c|c|c|c|}
\hline \multirow[b]{2}{*}{ Wall } & \multirow[b]{2}{*}{$H_{i}(m)$} & \multirow[b]{2}{*}{$D_{i}(m)$} & \multirow[b]{2}{*}{$t_{i}(m)$} & \multirow[b]{2}{*}{$A_{i}\left(m^{2}\right)$} & \multirow[b]{2}{*}{$H_{i} / D_{i}$} & \multicolumn{3}{|c|}{ Goel \& Chopra (1998) } & \multicolumn{2}{|c|}{$\begin{array}{c}\text { Wallace \& Moehle } \\
(1992)\end{array}$} & \multirow{2}{*}{$\begin{array}{c}\begin{array}{c}\text { Lee et al } \\
(2000)\end{array} \\
L_{w}\left(m^{-1}\right)\end{array}$} & \multicolumn{2}{|c|}{ Morales (2000) } \\
\hline & & & & & & $A_{e i}\left(m^{2}\right)$ & $A_{e}\left(m^{2}\right)$ & $A_{e}\left(\% A_{B}\right)$ & $(h / D)_{\min }$ & $p$ & & $I_{i}\left(m^{4}\right)$ & $I\left(m^{4}\right)$ \\
\hline W1 & 56 & 12.497 & 0.406 & 5.07 & 4.48 & 0.2872 & \multirow{3}{*}{0.5969} & \multirow{3}{*}{0.0456} & \multirow{3}{*}{4.48} & \multirow{3}{*}{0.0091} & \multirow{3}{*}{0.0235} & 66.03 & \multirow{3}{*}{137.00} \\
\hline W2 & 56 & 5.791 & 0.305 & 1.77 & 9.67 & 0.0225 & & & & & & 4.94 & \\
\hline W3 & 56 & 12.497 & 0.406 & 5.07 & 4.48 & 0.2872 & & & & & & 66.03 & \\
\hline
\end{tabular}

\section{Longitudinal direction}

Coupled shear walls 


\section{Building ID: RCSW-2}

\section{Storeys: 49}

Height: $195 \mathrm{~m}$

Area: $2599 \mathrm{~m}^{2}$

\section{Transverse direction}

Uncoupled shear walls

\begin{tabular}{|c|c|c|c|c|c|c|c|c|c|c|c|c|c|}
\hline \multirow[b]{2}{*}{ Wall } & \multirow[b]{2}{*}{$H_{i}(m)$} & \multirow[b]{2}{*}{$D_{i}(m)$} & \multirow[b]{2}{*}{$t_{i}(m)$} & \multirow[b]{2}{*}{$A_{i}\left(m^{2}\right)$} & \multirow[b]{2}{*}{$H_{i} / D_{i}$} & \multicolumn{3}{|c|}{ Goel \& Chopra (1998) } & \multicolumn{2}{|c|}{$\begin{array}{c}\text { Wallace \& Moehle } \\
(1992)\end{array}$} & \multirow{2}{*}{$\begin{array}{c}\begin{array}{c}\text { Lee et al } \\
(2000)\end{array} \\
L_{w}\left(m^{-1}\right)\end{array}$} & \multicolumn{2}{|c|}{ Morales (2000) } \\
\hline & & & & & & $A_{\mathrm{ei}}\left(m^{2}\right)$ & $A_{e}\left(m^{2}\right)$ & $A_{e}\left(\% A_{B}\right)$ & $(h / D)_{\min }$ & $p$ & & $I_{i}\left(m^{4}\right)$ & $I\left(m^{4}\right)$ \\
\hline W13 & 195 & 9.100 & 0.500 & 4.55 & 21.43 & 0.0119 & \multirow{6}{*}{0.0714} & \multirow{6}{*}{0.0027} & \multirow{6}{*}{21.43} & \multirow{6}{*}{0.0105} & \multirow{6}{*}{0.0210} & 31.40 & \multirow{6}{*}{188.39} \\
\hline W14 & 195 & 9.100 & 0.500 & 4.55 & 21.43 & 0.0119 & & & & & & 31.40 & \\
\hline W15 & 195 & 9.100 & 0.500 & 4.55 & 21.43 & 0.0119 & & & & & & 31.40 & \\
\hline W16 & 195 & 9.100 & 0.500 & 4.55 & 21.43 & 0.0119 & & & & & & 31.40 & \\
\hline W17 & 195 & 9.100 & 0.500 & 4.55 & 21.43 & 0.0119 & & & & & & 31.40 & \\
\hline W18 & 195 & 9.100 & 0.500 & 4.55 & 21.43 & 0.0119 & & & & & & 31.40 & \\
\hline
\end{tabular}

\section{Longitudinal direction}

Coupled shear walls 


\section{Building ID: RCSW-6}

Storeys: 12

Height: $43 \mathrm{~m}$

Area: $791 \mathrm{~m}^{2}$

\section{Transverse direction}

Coupled shear walls

\section{Longitudinal direction}

Uncoupled shear walls

\begin{tabular}{|c|c|c|c|c|c|c|c|c|c|c|c|c|c|}
\hline \multirow[b]{2}{*}{ Wall } & \multirow[b]{2}{*}{$H_{i}(m)$} & \multirow[b]{2}{*}{$D_{i}(m)$} & \multirow[b]{2}{*}{$t_{i}(m)$} & \multirow[b]{2}{*}{$A_{i}\left(m^{2}\right)$} & \multirow[b]{2}{*}{$H_{i} / D_{i}$} & \multicolumn{3}{|c|}{ Goel \& Chopra (1998) } & \multicolumn{2}{|c|}{$\begin{array}{c}\text { Wallace \& Moehle } \\
(1992)\end{array}$} & \multirow{2}{*}{$\begin{array}{c}\begin{array}{c}\text { Lee et al } \\
(2000)\end{array} \\
\mathrm{L}_{\mathrm{w}}\left(\mathrm{m}^{-1}\right)\end{array}$} & \multicolumn{2}{|c|}{ Morales (2000) } \\
\hline & & & & & & $A_{e i}\left(m^{2}\right)$ & $A_{e}\left(m^{2}\right)$ & $A_{e}\left(\% A_{B}\right)$ & $(\mathrm{h} / \mathrm{D})_{\min }$ & $p$ & & $I_{i}\left(m^{4}\right)$ & $I\left(m^{4}\right)$ \\
\hline W1 & 43 & 8.660 & 0.305 & 2.64 & 4.97 & 0.1231 & \multirow{2}{*}{0.2461} & \multirow{2}{*}{0.0311} & \multirow{2}{*}{4.97} & \multirow{2}{*}{0.0067} & \multirow{2}{*}{0.0219} & 16.51 & \multirow{2}{*}{33.01} \\
\hline W2 & 43 & 8.660 & 0.305 & 2.64 & 4.97 & 0.1231 & & & & & & 16.51 & \\
\hline
\end{tabular}




\section{Building ID: RCSW-7}

Storeys: 6

Height: $24 \mathrm{~m}$

Area: $1327 \mathrm{~m}^{2}$

\section{Transverse direction}

Coupled and uncoupled shear walls

\section{Longitudinal direction}

Uncoupled shear walls

\begin{tabular}{|c|c|c|c|c|c|c|c|c|c|c|c|c|c|}
\hline \multirow[b]{2}{*}{ Wall } & \multirow[b]{2}{*}{$H_{i}(m)$} & \multirow[b]{2}{*}{$D_{i}(m)$} & \multirow[b]{2}{*}{$t_{i}(m)$} & \multirow[b]{2}{*}{$A_{i}\left(m^{2}\right)$} & \multirow[b]{2}{*}{$H_{i} / D_{i}$} & \multicolumn{3}{|c|}{ Goel \& Chopra (1998) } & \multicolumn{2}{|c|}{$\begin{array}{c}\text { Wallace \& Moehle } \\
(1992)\end{array}$} & \multirow{2}{*}{$\begin{array}{c}\begin{array}{c}\text { Lee et al } \\
(2000)\end{array} \\
L_{w}\left(m^{-1}\right)\end{array}$} & \multicolumn{2}{|c|}{ Morales (2000) } \\
\hline & & & & & & $A_{e i}\left(m^{2}\right)$ & $A_{e}\left(m^{2}\right)$ & $A_{e}\left(\% A_{B}\right)$ & $(h / D)_{\min }$ & $p$ & & $I_{i}\left(m^{4}\right)$ & $I\left(m^{4}\right)$ \\
\hline W1 & 24 & 6.600 & 0.250 & 1.65 & 3.64 & 0.1378 & \multirow{6}{*}{0.3142} & \multirow{6}{*}{0.0237} & \multirow{6}{*}{3.64} & \multirow{6}{*}{0.0046} & \multirow{6}{*}{0.0169} & 5.99 & \multirow{6}{*}{13.54} \\
\hline W2 & 24 & 6.600 & 0.250 & 1.65 & 3.64 & 0.1378 & & & & & & 5.99 & \\
\hline W3 & 24 & 2.990 & 0.300 & 0.90 & 8.03 & 0.0165 & & & & & & 0.67 & \\
\hline W4 & 24 & 2.990 & 0.300 & 0.90 & 8.03 & 0.0165 & & & & & & 0.67 & \\
\hline W5 & 24 & 1.400 & 0.300 & 0.42 & 17.14 & 0.0017 & & & & & & 0.07 & \\
\hline W6 & 24 & 1.850 & 0.300 & 0.56 & 12.97 & 0.0039 & & & & & & 0.16 & \\
\hline
\end{tabular}




\section{Building ID: RCSW-8}

Storeys: 6

Height: $23 \mathrm{~m}$

Area: $1805 \mathrm{~m}^{2}$

\section{Transverse direction}

Uncoupled shear walls

\begin{tabular}{|c|c|c|c|c|c|c|c|c|c|c|c|c|c|}
\hline \multirow[b]{2}{*}{ Wall } & \multirow[b]{2}{*}{$H_{i}(m)$} & \multirow[b]{2}{*}{$D_{i}(m)$} & \multirow[b]{2}{*}{$t_{i}(m)$} & \multirow[b]{2}{*}{$A_{i}\left(m^{2}\right)$} & \multirow[b]{2}{*}{$H_{i} / D_{i}$} & \multicolumn{3}{|c|}{ Goel \& Chopra (1998) } & \multicolumn{2}{|c|}{$\begin{array}{c}\text { Wallace \& Moehle } \\
(1992)\end{array}$} & \multirow{2}{*}{$\begin{array}{c}\begin{array}{c}\text { Lee et al } \\
(2000)\end{array} \\
L_{w}\left(m^{-1}\right)\end{array}$} & \multicolumn{2}{|c|}{ Morales (2000) } \\
\hline & & & & & & $A_{e i}\left(m^{2}\right)$ & $A_{e}\left(m^{2}\right)$ & $A_{e}\left(\% A_{B}\right)$ & $(h / D)_{\min }$ & $p$ & & $I_{i}\left(m^{4}\right)$ & $I\left(\mathrm{~m}^{4}\right)$ \\
\hline W1 & 23 & 6.706 & 0.254 & 1.70 & 3.43 & 0.1582 & \multirow{6}{*}{0.6354} & \multirow{6}{*}{0.0352} & \multirow{6}{*}{3.43} & \multirow{6}{*}{0.0041} & \multirow{6}{*}{0.0163} & 6.38 & \multirow{6}{*}{25.62} \\
\hline W2 & 23 & 6.706 & 0.254 & 1.70 & 3.43 & 0.1582 & & & & & & 6.38 & \\
\hline W3 & 23 & 6.706 & 0.254 & 1.70 & 3.43 & 0.1582 & & & & & & 6.38 & \\
\hline W4 & 23 & 6.706 & 0.254 & 1.70 & 3.43 & 0.1582 & & & & & & 6.38 & \\
\hline W5 & 23 & 1.270 & 0.254 & 0.32 & 18.11 & 0.0012 & & & & & & 0.04 & \\
\hline W6 & 23 & 1.270 & 0.254 & 0.32 & 18.11 & 0.0012 & & & & & & 0.04 & \\
\hline
\end{tabular}

\section{Longitudinal direction}

Coupled and uncoupled shear walls 
Building ID: RCSW-13

Storeys: 12

Height: $45 \mathrm{~m}$

Area: $1723 \mathrm{~m}^{2}$

\section{Transverse direction}

Uncoupled shear walls

\begin{tabular}{|c|c|c|c|c|c|c|c|c|c|c|c|c|c|}
\hline \multirow[b]{2}{*}{ Wall } & \multirow[b]{2}{*}{$H_{i}(m)$} & \multirow[b]{2}{*}{$D_{i}(m)$} & \multirow[b]{2}{*}{$t_{i}(m)$} & \multirow[b]{2}{*}{$A_{i}\left(m^{2}\right)$} & \multirow[b]{2}{*}{$H_{i} / D_{i}$} & \multicolumn{3}{|c|}{ Goel \& Chopra (1998) } & \multicolumn{2}{|c|}{$\begin{array}{c}\text { Wallace \& Moehle } \\
(1992)\end{array}$} & \multirow{2}{*}{$\begin{array}{c}\begin{array}{c}\text { Lee et al } \\
(2000)\end{array} \\
L_{w}\left(m^{-1}\right)\end{array}$} & \multicolumn{2}{|c|}{ Morales (2000) } \\
\hline & & & & & & $A_{e i}\left(m^{2}\right)$ & $A_{e}\left(m^{2}\right)$ & $A_{e}\left(\% A_{B}\right)$ & $(h / D)_{\min }$ & $p$ & & $I_{i}\left(m^{4}\right)$ & $I\left(\mathrm{~m}^{4}\right)$ \\
\hline W1 & 45 & 4.159 & 0.305 & 1.27 & 10.82 & 0.0129 & \multirow{6}{*}{0.0453} & \multirow{6}{*}{0.0026} & \multirow{6}{*}{10.82} & \multirow{6}{*}{0.0036} & \multirow{6}{*}{0.0118} & 1.83 & \multirow{6}{*}{6.40} \\
\hline W2 & 45 & 4.159 & 0.305 & 1.27 & 10.82 & 0.0129 & & & & & & 1.83 & \\
\hline W3 & 45 & 2.896 & 0.305 & 0.88 & 15.54 & 0.0044 & & & & & & 0.62 & \\
\hline W4 & 45 & 3.099 & 0.305 & 0.94 & 14.52 & 0.0054 & & & & & & 0.76 & \\
\hline W5 & 45 & 2.896 & 0.305 & 0.88 & 15.54 & 0.0044 & & & & & & 0.62 & \\
\hline W6 & 45 & 3.099 & 0.305 & 0.94 & 14.52 & 0.0054 & & & & & & 0.76 & \\
\hline
\end{tabular}

\section{Longitudinal direction}

Coupled and uncoupled shear walls 


\section{Building ID: RCSW-16}

Storeys: 6

Height: $20 \mathrm{~m}$

Area: $809 \mathrm{~m}^{2}$

\section{Transverse direction}

Uncoupled shear walls

\begin{tabular}{|c|c|c|c|c|c|c|c|c|c|c|c|c|c|}
\hline \multirow[b]{2}{*}{ Wall } & \multirow[b]{2}{*}{$H_{i}(m)$} & \multirow[b]{2}{*}{$D_{i}(m)$} & \multirow[b]{2}{*}{$t_{i}(m)$} & \multirow[b]{2}{*}{$A_{i}\left(m^{2}\right)$} & \multirow[b]{2}{*}{$H_{i} / D_{i}$} & \multicolumn{3}{|c|}{ Goel \& Chopra (1998) } & \multicolumn{2}{|c|}{$\begin{array}{c}\text { Wallace \& Moehle } \\
(1992)\end{array}$} & \multirow{2}{*}{$\begin{array}{c}\text { Lee et al } \\
(2000) \\
L_{w}\left(m^{-1}\right)\end{array}$} & \multicolumn{2}{|c|}{ Morales (2000) } \\
\hline & & & & & & $A_{e i}\left(m^{2}\right)$ & $A_{e}\left(m^{2}\right)$ & $A_{e}\left(\% A_{B}\right)$ & $(h / D)_{\min }$ & $p$ & & $I_{i}\left(m^{4}\right)$ & $I\left(m^{4}\right)$ \\
\hline W2 & 20 & 3.310 & 0.300 & 0.99 & 6.04 & 0.0317 & \multirow{5}{*}{0.2893} & \multirow{5}{*}{0.0358} & \multirow{5}{*}{3.71} & \multirow{5}{*}{0.0069} & \multirow{5}{*}{0.0251} & 0.91 & \multirow{5}{*}{8.53} \\
\hline W3 & 20 & 3.350 & 0.300 & 1.01 & 5.97 & 0.0329 & & & & & & 0.94 & \\
\hline W4 & 20 & 3.310 & 0.300 & 0.99 & 6.04 & 0.0317 & & & & & & 0.91 & \\
\hline W5 & 20 & 4.940 & 0.250 & 1.24 & 4.05 & 0.0846 & & & & & & 2.51 & \\
\hline W6 & 20 & 5.390 & 0.250 & 1.35 & 3.71 & 0.1084 & & & & & & 3.26 & \\
\hline
\end{tabular}

\section{Longitudinal direction}

Uncoupled shear walls

\begin{tabular}{|c|c|c|c|c|c|c|c|c|c|c|c|c|c|}
\hline \multirow[b]{2}{*}{ Wall } & \multirow[b]{2}{*}{$H_{i}(m)$} & \multirow[b]{2}{*}{$D_{i}(m)$} & \multirow[b]{2}{*}{$t_{i}(m)$} & \multirow[b]{2}{*}{$A_{i}\left(m^{2}\right)$} & \multirow[b]{2}{*}{$H_{i} / D_{i}$} & \multicolumn{3}{|c|}{ Goel \& Chopra (1998) } & \multicolumn{2}{|c|}{$\begin{array}{c}\text { Wallace \& Moehle } \\
(1992)\end{array}$} & \multirow{2}{*}{$\begin{array}{c}\begin{array}{c}\text { Lee et al } \\
(2000)\end{array} \\
L_{w}\left(m^{-1}\right)\end{array}$} & \multicolumn{2}{|c|}{ Morales (2000) } \\
\hline & & & & & & $A_{e i}\left(m^{2}\right)$ & $A_{e}\left(m^{2}\right)$ & $A_{e}\left(\% A_{B}\right)$ & $(h / D)_{\min }$ & p & & $I_{i}\left(m^{4}\right)$ & $I\left(\mathrm{~m}^{4}\right)$ \\
\hline W1 & 20 & 8.735 & 0.250 & 2.18 & 2.29 & 0.4081 & 0.4081 & 0.0504 & 2.29 & 0.0027 & 0.0108 & 13.89 & 13.89 \\
\hline
\end{tabular}


Building ID: RCSW-17

Storeys: 22

Height: $75 \mathrm{~m}$

Area: $2155 \mathrm{~m}^{2}$

\section{Transverse direction}

Coupled shear walls

\section{Longitudinal direction}

Uncoupled shear walls

\begin{tabular}{|c|c|c|c|c|c|c|c|c|c|c|c|c|c|}
\hline \multirow[b]{2}{*}{ Wall } & \multirow[b]{2}{*}{$H_{i}(m)$} & \multirow[b]{2}{*}{$D_{i}(m)$} & \multirow[b]{2}{*}{$t_{i}(m)$} & \multirow[b]{2}{*}{$A_{i}\left(m^{2}\right)$} & \multirow[b]{2}{*}{$H_{i} / D_{i}$} & \multicolumn{3}{|c|}{ Goel \& Chopra (1998) } & \multicolumn{2}{|c|}{$\begin{array}{c}\text { Wallace \& Moehle } \\
(1992)\end{array}$} & \multirow{2}{*}{$\begin{array}{c}\text { Lee et al } \\
(2000) \\
L_{w}\left(m^{-1}\right)\end{array}$} & \multicolumn{2}{|c|}{ Morales (2000) } \\
\hline & & & & & & $A_{e i}\left(m^{2}\right)$ & $A_{e}\left(m^{2}\right)$ & $A_{e}\left(\% A_{B}\right)$ & $(h / D)_{\min }$ & $p$ & & $I_{i}\left(m^{4}\right)$ & $I\left(m^{4}\right)$ \\
\hline W3 & 75 & 3.594 & 0.254 & 0.91 & 20.87 & 0.0025 & \multirow{4}{*}{0.3802} & \multirow{4}{*}{0.0176} & \multirow{4}{*}{5.20} & \multirow{4}{*}{0.0049} & \multirow{4}{*}{0.0166} & 0.98 & \multirow{4}{*}{154.42} \\
\hline W4 & 75 & 14.427 & 0.305 & 4.40 & 5.20 & 0.1878 & & & & & & 76.32 & \\
\hline W4 & 75 & 14.427 & 0.305 & 4.40 & 5.20 & 0.1878 & & & & & & 76.32 & \\
\hline W5 & 75 & 3.353 & 0.254 & 0.85 & 22.37 & 0.0020 & & & & & & 0.80 & \\
\hline
\end{tabular}




\section{Building ID: RCSW-18}

\section{Storeys: 6}

\section{Height: $25 \mathrm{~m}$}

Area: $730 \mathrm{~m}^{2}$

Transverse direction (Uncoupled shear walls)

\begin{tabular}{|c|c|c|c|c|c|c|c|c|c|c|c|c|c|}
\hline \multirow[b]{2}{*}{ Wall } & \multirow[b]{2}{*}{$H_{i}(m)$} & \multirow[b]{2}{*}{$D_{i}(m)$} & \multirow[b]{2}{*}{$t_{i}(m)$} & \multirow[b]{2}{*}{$A_{i}\left(m^{2}\right)$} & \multirow[b]{2}{*}{$H_{i} / D_{i}$} & \multicolumn{3}{|c|}{ Goel \& Chopra (1998) } & \multicolumn{2}{|c|}{$\begin{array}{c}\text { Wallace \& Moehle } \\
(1992)\end{array}$} & \multirow{2}{*}{$\begin{array}{c}\text { Lee et al } \\
(2000) \\
L_{w}\left(m^{-1}\right)\end{array}$} & \multicolumn{2}{|c|}{ Morales (2000) } \\
\hline & & & & & & $A_{e i}\left(m^{2}\right)$ & $A_{e}\left(m^{2}\right)$ & $A_{e}\left(\% A_{B}\right)$ & $(h / D)_{\min }$ & $p$ & & $I_{i}\left(m^{4}\right)$ & $I\left(m^{4}\right)$ \\
\hline W1 & 25 & 3.112 & 0.203 & 0.63 & 8.03 & 0.0116 & \multirow{10}{*}{0.1510} & \multirow{10}{*}{0.0207} & \multirow{10}{*}{7.37} & \multirow{10}{*}{0.0101} & \multirow{10}{*}{0.0449} & 0.51 & \multirow{10}{*}{6.66} \\
\hline W2 & 25 & 3.391 & 0.254 & 0.86 & 7.37 & 0.0187 & & & & & & 0.83 & \\
\hline W3 & 25 & 3.391 & 0.254 & 0.86 & 7.37 & 0.0187 & & & & & & 0.83 & \\
\hline W4 & 25 & 3.391 & 0.203 & 0.69 & 7.37 & 0.0149 & & & & & & 0.66 & \\
\hline W5 & 25 & 3.112 & 0.203 & 0.63 & 8.03 & 0.0116 & & & & & & 0.51 & \\
\hline W6 & 25 & 3.112 & 0.203 & 0.63 & 8.03 & 0.0116 & & & & & & 0.51 & \\
\hline W7 & 25 & 3.391 & 0.203 & 0.69 & 7.37 & 0.0149 & & & & & & 0.66 & \\
\hline W8 & 25 & 3.391 & 0.254 & 0.86 & 7.37 & 0.0187 & & & & & & 0.83 & \\
\hline W9 & 25 & 3.391 & 0.254 & 0.86 & 7.37 & 0.0187 & & & & & & 0.83 & \\
\hline W10 & 25 & 3.112 & 0.203 & 0.63 & 8.03 & 0.0116 & & & & & & 0.51 & \\
\hline
\end{tabular}

\section{Longitudinal direction (Uncoupled shear walls)}

Uncoupled shear walls

\begin{tabular}{|c|c|c|c|c|c|c|c|c|c|c|c|c|c|}
\hline \multirow[b]{2}{*}{ Wall } & \multirow[b]{2}{*}{$H_{i}(m)$} & \multirow[b]{2}{*}{$D_{i}(m)$} & \multirow[b]{2}{*}{$t_{i}(m)$} & \multirow[b]{2}{*}{$A_{i}\left(m^{2}\right)$} & \multirow[b]{2}{*}{$H_{i} / D_{i}$} & \multicolumn{3}{|c|}{ Goel \& Chopra (1998) } & \multicolumn{2}{|c|}{$\begin{array}{c}\text { Wallace \& Moehle } \\
(1992)\end{array}$} & \multirow{2}{*}{$\begin{array}{c}\begin{array}{c}\text { Lee et al } \\
(2000)\end{array} \\
L_{w}\left(m^{-1}\right)\end{array}$} & \multicolumn{2}{|c|}{ Morales (2000) } \\
\hline & & & & & & $A_{e i}\left(m^{2}\right)$ & $A_{e}\left(m^{2}\right)$ & $A_{e}\left(\% A_{B}\right)$ & $(h / D)_{\min }$ & $p$ & & $I_{i}\left(m^{4}\right)$ & $I\left(m^{4}\right)$ \\
\hline W11 & 25 & 7.957 & 0.203 & 1.62 & 3.14 & 0.1759 & \multirow{4}{*}{0.7035} & \multirow{4}{*}{0.0964} & \multirow{4}{*}{3.14} & \multirow{4}{*}{0.0089} & \multirow{4}{*}{0.0436} & 8.53 & \multirow{4}{*}{34.12} \\
\hline W12 & 25 & 7.957 & 0.203 & 1.62 & 3.14 & 0.1759 & & & & & & 8.53 & \\
\hline W13 & 25 & 7.957 & 0.203 & 1.62 & 3.14 & 0.1759 & & & & & & 8.53 & \\
\hline W14 & 25 & 7.957 & 0.203 & 1.62 & 3.14 & 0.1759 & & & & & & 8.53 & \\
\hline
\end{tabular}


Building ID: RCSW-20

Storeys: 22

Height: $77 \mathrm{~m}$

Area: $1709 \mathrm{~m}^{2}$

\section{Transverse direction}

Coupled shear walls

\section{Longitudinal direction}

Uncoupled shear walls

\begin{tabular}{|c|c|c|c|c|c|c|c|c|c|c|c|c|c|}
\hline \multirow[b]{2}{*}{ Wall } & \multirow[b]{2}{*}{$H_{i}(\mathbf{m})$} & \multirow[b]{2}{*}{$D_{i}(m)$} & \multirow[b]{2}{*}{$t_{i}(m)$} & \multirow[b]{2}{*}{$A_{i}\left(m^{2}\right)$} & \multirow[b]{2}{*}{$H_{i} / D_{i}$} & \multicolumn{3}{|c|}{ Goel \& Chopra (1998) } & \multicolumn{2}{|c|}{$\begin{array}{c}\text { Wallace \& Moehle } \\
(1992)\end{array}$} & \multirow{2}{*}{$\begin{array}{c}\begin{array}{c}\text { Lee et al } \\
(2000)\end{array} \\
\mathrm{L}_{\mathrm{w}}\left(\mathrm{m}^{-1}\right)\end{array}$} & \multicolumn{2}{|c|}{ Morales (2000) } \\
\hline & & & & & & $A_{e i}\left(m^{2}\right)$ & $A_{e}\left(m^{2}\right)$ & $A_{e}\left(\% A_{B}\right)$ & $(h / D)_{\min }$ & $p$ & & $I_{i}\left(m^{4}\right)$ & $I\left(\mathrm{~m}^{4}\right)$ \\
\hline W1 & 77 & 5.436 & 0.203 & 1.10 & 14.16 & 0.0066 & \multirow{4}{*}{0.2289} & \multirow{4}{*}{0.0134} & \multirow{4}{*}{6.77} & \multirow{4}{*}{0.0057} & \multirow{4}{*}{0.0232} & 2.72 & \multirow{4}{*}{96.28} \\
\hline W2 & 77 & 11.379 & 0.254 & 2.89 & 6.77 & 0.0741 & & & & & & 31.19 & \\
\hline W3 & 77 & 11.379 & 0.254 & 2.89 & 6.77 & 0.0741 & & & & & & 31.19 & \\
\hline W4 & 77 & 11.379 & 0.254 & 2.89 & 6.77 & 0.0741 & & & & & & 31.19 & \\
\hline
\end{tabular}




\section{Appendix G:}

Data used for evaluation of alternative fundamental period formulae 
Table G-1: Data for Goel and Chopra formula for selected buildings in Montréal

\begin{tabular}{|c|c|c|c|c|c|c|c|c|c|c|c|}
\hline \multirow[b]{2}{*}{ ID } & \multicolumn{2}{|c|}{ Type } & \multirow{2}{*}{$\begin{array}{l}\text { Number of } \\
\text { Storeys }\end{array}$} & \multirow[b]{2}{*}{ Height (m) } & \multirow[b]{2}{*}{$A_{B}\left(m^{2}\right)$} & \multicolumn{2}{|c|}{$A_{e}\left(\% A_{B}\right)$} & \multicolumn{2}{|c|}{$h /\left(A_{e}^{0.5}\right)$} & \multicolumn{2}{|c|}{ Measured period (s) } \\
\hline & Trans & Long & & & & Trans & Long & Trans & Long & Trans & Long \\
\hline RCSW-1 & USW & CSW & 15 & 56 & 1310 & 0.0456 & NA & 262 & NA & 0.99 & NA \\
\hline RCSW-2 & USW & CSW & 49 & 195 & 2599 & 0.0027 & NA & 3719 & NA & 4.35 & NA \\
\hline RCSW-6 & CSW & USW & 12 & 43 & 791 & NA & 0.0311 & NA & 244 & NA & 0.77 \\
\hline RCSW-7 & CUSW & USW & 6 & 24 & 1327 & NA & 0.0237 & NA & 156 & NA & 0.38 \\
\hline RCSW-8 & USW & CUSW & 6 & 23 & 1805 & 0.0352 & NA & 123 & NA & 0.46 & NA \\
\hline RCSW-13 & USW & Cusw & 12 & 45 & 1723 & 0.0026 & $\mathrm{NA}$ & 877 & NA & 0.99 & NA \\
\hline RCSW-16 & USW & USW & 6 & 20 & 809 & 0.0358 & 0.0504 & 106 & 89 & 0.40 & 0.36 \\
\hline RCSW-17 & CSW & USW & 22 & 75 & 2155 & NA & 0.0176 & NA & 565 & NA & 1.75 \\
\hline RCSW-18 & USW & USW & 6 & 25 & 730 & 0.0207 & 0.0964 & 174 & 81 & 0.41 & 0.29 \\
\hline RCSW-20 & CSW & USW & 22 & 77 & 1709 & NA & 0.0134 & NA & 665 & NA & 1.72 \\
\hline \multicolumn{12}{|c|}{$\begin{array}{l}\text { CSW: Coupled } \\
\text { CUSW: Coupled } \\
\text { USW: Uncoup } \\
\text { NA: } \quad \text { Lateral I }\end{array}$} \\
\hline
\end{tabular}

Table G-2: Data for Goel and Chopra formula for selected buildings in California (from Goel and Chopra (1998))

\begin{tabular}{|c|c|c|c|c|c|c|c|c|c|c|c|}
\hline \multirow[b]{2}{*}{ ID } & \multicolumn{2}{|c|}{ Type } & \multirow{2}{*}{$\begin{array}{l}\text { Number of } \\
\text { Storeys }\end{array}$} & \multirow[b]{2}{*}{ Height (m) } & \multirow[b]{2}{*}{$A_{B}\left(m^{2}\right)$} & \multicolumn{2}{|c|}{$A_{e}\left(\% A_{B}\right)$} & \multicolumn{2}{|c|}{$h /\left(A_{e}^{0.5}\right)$} & \multicolumn{2}{|c|}{ Measured period (s) } \\
\hline & Trans & Long & & & & Trans & Long & Trans & Long & Trans & Long \\
\hline C24385 & USW & USW & 10 & 26.8 & 1498 & 0.2019 & 0.1978 & 60 & 60 & 0.56 & 0.60 \\
\hline C24385 & USW & USW & 10 & 26.8 & 1498 & 0.2019 & 0.1978 & 60 & 60 & 0.51 & 0.57 \\
\hline C24468 & USW & USW & 8 & 38.7 & 901 & 0.0416 & 0.0319 & 190 & 217 & 1.62 & 1.54 \\
\hline C24601 & USW & USW & 17 & 45.6 & 1687 & 0.1131 & 0.0765 & 136 & 165 & 1.05 & 1.18 \\
\hline $\mathrm{C} 24601$ & USW & USW & 17 & 45.6 & 1687 & 0.1131 & 0.0765 & 136 & 165 & 1.00 & 1.00 \\
\hline C12284 & USW & USW & 4 & 15.3 & 1003 & 0.0662 & 0.0646 & 59 & 60 & 0.60 & 0.50 \\
\hline C58334 & USW & USW & 3 & 11.0 & 754 & 0.1579 & 0.1579 & 28 & 28 & 0.18 & 0.18 \\
\hline C58348 & USW & USW & 3 & 12.4 & 937 & 0.0603 & 0.1346 & 50 & 34 & 0.46 & 0.38 \\
\hline C58394 & USW & USW & 9 & 31.7 & 1498 & 0.0228 & 0.0397 & 210 & 159 & 1.30 & 1.20 \\
\hline C58394 & USW & USW & 9 & 31.7 & 1498 & 0.0228 & 0.0397 & 210 & 159 & 1.45 & 1.00 \\
\hline C57355 & USW & MRF & 10 & 37.8 & 1589 & 0.3309 & MRF & 66 & MRF & 0.75 & MRF \\
\hline C57355 & USW & MRF & 10 & 37.8 & 1589 & 0.3309 & MRF & 66 & MRF & 0.61 & MRF \\
\hline
\end{tabular}


Table G-2: Data for Goel and Chopra formula for selected buildings in California (from Goel and Chopra (1998)) (... Continued)

\begin{tabular}{|c|c|c|c|c|c|c|c|c|c|c|c|}
\hline \multirow[b]{2}{*}{ ID } & \multicolumn{2}{|c|}{ Type } & \multirow{2}{*}{$\begin{array}{c}\text { Number of } \\
\text { storeys }\end{array}$} & \multirow[b]{2}{*}{ Height $(\mathrm{m})$} & \multirow[b]{2}{*}{$A_{B}\left(m^{2}\right)$} & \multicolumn{2}{|c|}{$A_{e}\left(\% A_{B}\right)$} & \multicolumn{2}{|c|}{$h /\left(A_{e}^{0.5}\right)$} & \multicolumn{2}{|c|}{ Measured period (s) } \\
\hline & Trans & Long & & & & Trans & Long & Trans & Long & Trans & Long \\
\hline C57355 & USW & MRF & 10 & 37.8 & 1589 & 0.3309 & MRF & 66 & MRF & 0.61 & MRF \\
\hline C57356 & USW & USW & 10 & 29.3 & 1249 & 0.2563 & 0.2120 & 58 & 64 & 0.43 & 0.73 \\
\hline C57356 & USW & USW & 10 & 29.3 & 1249 & 0.2563 & 0.2120 & 58 & 64 & 0.42 & 0.70 \\
\hline C57356 & USW & USW & 10 & 29.3 & 1249 & 0.2563 & 0.2120 & 58 & 64 & 0.43 & 0.65 \\
\hline C57356 & USW & USW & 10 & 29.3 & 1249 & 0.2563 & 0.2120 & 58 & 64 & 0.41 & 0.63 \\
\hline
\end{tabular}


Table G-3: Data for Wallace and Moehle formula for selected buildings in Montréal

\begin{tabular}{|c|c|c|c|c|c|c|c|c|c|c|c|c|c|}
\hline \multirow[b]{2}{*}{ ID } & \multicolumn{2}{|c|}{ Type } & \multirow{2}{*}{$\begin{array}{c}\begin{array}{c}\text { Number } \\
\text { of } \\
\text { Storeys }\end{array} \\
\end{array}$} & \multirow{2}{*}{$\begin{array}{c}\text { Height } \\
(\mathrm{m})\end{array}$} & \multirow[b]{2}{*}{$A_{B}\left(m^{2}\right)$} & \multicolumn{2}{|c|}{$(h / D)_{\min }$} & \multicolumn{2}{|c|}{$p$} & \multicolumn{2}{|c|}{$(h / D)_{\min }{ }^{*} n /\left(p^{0.5}\right)$} & \multicolumn{2}{|c|}{ Measured period (s) } \\
\hline & Trans & Long & & & & Trans & Long & Trans & Long & Trans & Long & Trans & Long \\
\hline RCSW-1 & USW & CSW & 15 & 56 & 1310 & 4.48 & NA & 0.0091 & NA & 705 & NA & 0.99 & NA \\
\hline RCSW-2 & USW & CSW & 49 & 195 & 2599 & 21.43 & NA & 0.0105 & NA & 10245 & NA & 4.35 & NA \\
\hline RCSW-6 & CSW & USW & 12 & 43 & 791 & NA & 4.97 & NA & 0.0067 & NA & 729 & NA & 0.77 \\
\hline RCSW-7 & CUSW & USW & 6 & 24 & 1327 & NA & 3.64 & NA & 0.0046 & NA & 323 & NA & 0.38 \\
\hline RCSW-8 & USW & CUSW & 6 & 23 & 1805 & 3.43 & NA & 0.0041 & NA & 320 & NA & 0.46 & NA \\
\hline RCSW-13 & USW & CUSW & 12 & 45 & 1723 & 10.82 & NA & 0.0036 & NA & 2166 & NA & 0.99 & NA \\
\hline RCSW-16 & USW & USW & 6 & 20 & 809 & 3.71 & 2.29 & 0.0069 & 0.0027 & 268 & 264 & 0.40 & 0.36 \\
\hline RCSW-17 & CSW & USW & 22 & 75 & 2155 & NA & 5.20 & NA & 0.0049 & NA & 1633 & NA & 1.75 \\
\hline RCSW-18 & USW & USW & 6 & 25 & 730 & 7.37 & 3.14 & 0.0101 & 0.0089 & 441 & 200 & 0.41 & 0.29 \\
\hline RCSW-20 & CSW & USW & 22 & 77 & 1709 & NA & 6.77 & NA & 0.0057 & NA & 1968 & NA & 1.72 \\
\hline
\end{tabular}

CSW: Coupled shear walls

CUSW: Coupled and uncoupled shear walls

USW: Uncoupled shear walls

NA: $\quad$ Lateral loads not resisted by uncoupled shear walls

$\mathrm{A}_{\mathrm{B}}: \quad$ Building plan area at the base of the building

$(\mathrm{h} / \mathrm{D})_{\text {min: }}$ Wall height to width ratio for the largest walls (minimum value)

p: $\quad$ Ratio of wall area to building plan area for walls aligned in direction under consideration

n: Number of storeys

Table G-4: Data for Wallace and Moehle formula for buildings in Chile and the U.S. (from Wallace and Moehle (1992), mostly adapted from Midorikawa (1990) and Calcagni Castillo (1988))

\begin{tabular}{|c|c|c|c|c|c|c|c|c|c|c|c|c|c|}
\hline \multirow[b]{2}{*}{ ID } & \multicolumn{2}{|c|}{ Type } & \multirow{2}{*}{$\begin{array}{c}\text { Number } \\
\text { of } \\
\text { Storeys }\end{array}$} & \multirow{2}{*}{$\begin{array}{c}\text { Height } \\
(\mathrm{m})\end{array}$} & \multirow[b]{2}{*}{$A_{B}\left(m^{2}\right)$} & \multicolumn{2}{|c|}{$(h / D)_{\min }$} & \multicolumn{2}{|c|}{$p$} & \multicolumn{2}{|c|}{$(h / D)_{\min }{ }^{*} n /\left(p^{0.5}\right)$} & \multicolumn{2}{|c|}{ Measured period (s) } \\
\hline & Trans & Long & & & & Trans & Long & Trans & Long & Trans & Long & Trans & Long \\
\hline Plaza del Mar & USW & USW & 23 & NA & NA & 7.0 & 7.0 & 0.035 & 0.044 & 861 & 768 & 1.58 & 1.34 \\
\hline Festival & USW & USW & 15 & NA & NA & 4.0 & 4.0 & 0.026 & 0.026 & 372 & 372 & 0.75 & 0.64 \\
\hline Acapulco & USW & USW & 15 & NA & NA & 7.0 & 5.0 & 0.025 & 0.038 & 664 & 385 & 1.07 & 0.79 \\
\hline Torres del Miramar & USW & USW & 22 & NA & NA & 6.0 & 6.0 & 0.028 & 0.028 & 789 & 789 & 1.07 & 1.06 \\
\hline Villa Real & USW & USW & 10 & NA & NA & 4.0 & 7.0 & 0.036 & 0.024 & 211 & 452 & 0.54 & 0.71 \\
\hline Torres del Sol & USW & USW & 22 & NA & NA & 8.0 & 7.0 & 0.034 & 0.034 & 954 & 835 & 1.03 & 1.01 \\
\hline Marina Real & USW & USW & 19 & NA & NA & 8.0 & 8.0 & 0.047 & 0.043 & 701 & 733 & 1.01 & 0.96 \\
\hline El Almendral & USW & USW & 23 & NA & NA & 6.0 & 7.0 & 0.023 & 0.018 & 910 & 1200 & 1.00 & 1.20 \\
\hline Building $1^{*}$ & USW & USW & 10 & NA & NA & 3.3 & 4.0 & 0.025 & 0.010 & 209 & 400 & 0.48 & 0.63 \\
\hline Building $2^{*}$ & USW & USW & 10 & NA & NA & 3.5 & 3.0 & 0.023 & 0.019 & 231 & 218 & 0.57 & 0.51 \\
\hline
\end{tabular}

* Period identified during moderate earthquake ground motions

NA: Information not available 
Table G-5: Data for Lee et al formula for selected buildings in Montréal

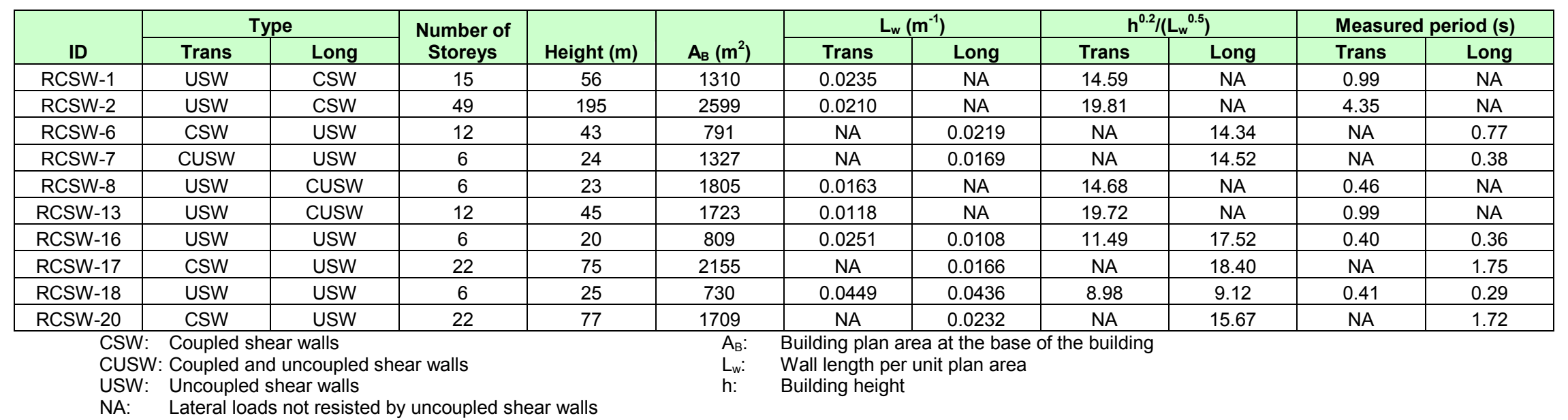

Table G-6: Data for Lee et al formula for selected buildings in Korea (from Lee et al (2000))

\begin{tabular}{|c|c|c|c|c|c|c|c|c|c|c|c|}
\hline \multirow[b]{2}{*}{ ID } & \multicolumn{2}{|c|}{ Type } & \multirow{2}{*}{$\begin{array}{l}\text { Number of } \\
\text { Storeys }\end{array}$} & \multirow[b]{2}{*}{ Height (m) } & \multirow[b]{2}{*}{$A_{B}\left(m^{2}\right)$} & \multicolumn{2}{|c|}{$L_{w}\left(m^{-1}\right)$} & \multicolumn{2}{|c|}{$h^{0.2} /\left(L_{w}{ }^{0.5}\right)$} & \multicolumn{2}{|c|}{ Measured period (s) } \\
\hline & Trans & Long & & & & Trans & Long & Trans & Long & Trans & Long \\
\hline 1 & USW & USW & 15 & 40.0 & NA & 0.28 & 0.15 & 3.95 & 5.40 & 0.71 & 1.92 \\
\hline 2 & USW & USW & 15 & 40.0 & NA & 0.26 & NA & 4.10 & NA & 1.08 & NA \\
\hline 3 & USW & USW & 20 & 53.5 & NA & 0.23 & 0.13 & 4.62 & 6.15 & 1.19 & 1.89 \\
\hline 4 & USW & USW & 20 & 53.5 & NA & 0.21 & 0.14 & 4.84 & 5.92 & 1.44 & 1.90 \\
\hline 5 & USW & USW & 20 & 53.5 & NA & NA & 0.14 & NA & 5.92 & NA & 1.93 \\
\hline 6 & USW & USW & 15 & 40.0 & NA & 0.20 & NA & 4.68 & NA & 1.27 & NA \\
\hline 7 & USW & USW & 15 & 40.0 & NA & NA & 0.12 & NA & 6.04 & NA & 2.22 \\
\hline 8 & USW & USW & 15 & 40.0 & NA & 0.25 & 0.13 & 4.18 & 5.80 & 1.16 & 1.86 \\
\hline 9 & USW & USW & 15 & 40.0 & NA & 0.27 & 0.13 & 4.02 & 5.80 & 1.09 & 1.66 \\
\hline 10 & USW & USW & 15 & 40.0 & NA & NA & 0.13 & NA & 5.80 & NA & 1.93 \\
\hline 11 & USW & USW & 20 & 53.5 & NA & NA & 0.13 & NA & 6.15 & NA & 2.11 \\
\hline 12 & USW & USW & 15 & 40.0 & NA & NA & 0.15 & NA & 5.40 & NA & 1.63 \\
\hline
\end{tabular}


Table G-6: Data for Lee et al formula for selected buildings in Korea (from Lee et al (2000)) (...Continued)

\begin{tabular}{|c|c|c|c|c|c|c|c|c|c|c|c|}
\hline \multirow[b]{2}{*}{ ID } & \multicolumn{2}{|c|}{ Type } & \multirow{2}{*}{$\begin{array}{c}\text { Number of } \\
\text { storeys }\end{array}$} & \multirow[b]{2}{*}{ Height (m) } & \multirow[b]{2}{*}{$A_{B}\left(m^{2}\right)$} & \multicolumn{2}{|c|}{$L_{w}\left(m^{-1}\right)$} & \multicolumn{2}{|c|}{$h^{0.2} /\left(L_{w}{ }^{0.5}\right)$} & \multicolumn{2}{|c|}{ Measured period (s) } \\
\hline & Trans & Long & & & & Trans & Long & Trans & Long & Trans & Long \\
\hline 13 & USW & USW & 15 & 40.0 & NA & 0.28 & 0.12 & 3.95 & 6.04 & 0.91 & 2.05 \\
\hline 14 & USW & USW & 20 & 53.5 & NA & 0.23 & 0.16 & 4.62 & 5.54 & 1.16 & 1.82 \\
\hline 15 & USW & USW & 20 & 53.5 & NA & NA & 0.16 & NA & 5.54 & NA & 1.95 \\
\hline 16 & USW & USW & 20 & 53.5 & NA & NA & 0.16 & NA & 5.54 & NA & 1.88 \\
\hline 17 & USW & USW & 20 & 53.5 & NA & 0.21 & 0.17 & 4.84 & 5.38 & 1.50 & 1.82 \\
\hline 18 & USW & USW & 20 & 53.5 & NA & NA & 0.17 & NA & 5.38 & NA & 1.76 \\
\hline 19 & USW & USW & 15 & 40.0 & NA & 0.29 & 0.15 & 3.88 & 5.40 & 0.90 & 1.91 \\
\hline 20 & USW & USW & 15 & 40.0 & NA & 0.30 & NA & 3.82 & NA & 0.86 & NA \\
\hline 21 & USW & USW & 15 & 40.0 & NA & 0.25 & 0.15 & 4.18 & 5.40 & 1.28 & 1.89 \\
\hline 22 & USW & USW & 15 & 40.0 & NA & 0.26 & NA & 4.10 & NA & 0.99 & NA \\
\hline 23 & USW & USW & 15 & 40.0 & NA & 0.28 & NA & 3.95 & NA & 1.16 & NA \\
\hline 24 & USW & USW & 15 & 40.0 & NA & 0.27 & 0.15 & 4.02 & 5.40 & 1.27 & 1.92 \\
\hline 25 & USW & USW & 15 & 40.0 & NA & NA & 0.17 & NA & 5.07 & NA & 1.79 \\
\hline 26 & USW & USW & 15 & 40.0 & NA & NA & 0.17 & NA & 5.07 & NA & 1.65 \\
\hline 27 & USW & USW & 18 & 48.1 & NA & NA & 0.11 & NA & 6.54 & NA & 1.81 \\
\hline 28 & USW & USW & 20 & 53.5 & NA & 0.28 & 0.16 & 4.19 & 5.54 & 1.17 & 1.85 \\
\hline 29 & USW & USW & 18 & 48.1 & NA & 0.23 & 0.15 & 4.52 & 5.60 & 1.23 & 1.88 \\
\hline 30 & USW & USW & 20 & 53.0 & NA & 0.19 & 0.14 & 5.08 & 5.91 & 1.12 & 1.88 \\
\hline 31 & USW & USW & 20 & 53.5 & NA & NA & 0.14 & NA & 5.92 & NA & 1.83 \\
\hline 32 & USW & USW & 20 & 53.5 & NA & 0.20 & 0.15 & 4.96 & 5.72 & 1.31 & 1.92 \\
\hline 33 & USW & USW & 20 & 53.5 & NA & 0.28 & 0.16 & 4.19 & 5.54 & 1.06 & 1.79 \\
\hline 34 & USW & USW & 22 & 58.9 & NA & 0.29 & 0.16 & 4.20 & 5.65 & 1.04 & 1.89 \\
\hline 35 & USW & USW & 25 & 67.0 & NA & 0.23 & 0.12 & 4.83 & 6.69 & 1.79 & 2.33 \\
\hline 36 & USW & USW & 25 & 67.0 & NA & 0.30 & NA & 4.23 & NA & 1.33 & NA \\
\hline 37 & USW & USW & 25 & 67.9 & NA & 0.24 & 0.12 & 4.75 & 6.71 & 1.39 & 2.56 \\
\hline 38 & USW & USW & 25 & 67.9 & NA & 0.28 & 0.16 & 4.39 & 5.81 & 1.59 & 2.04 \\
\hline 39 & USW & USW & 25 & 67.9 & NA & 0.25 & 0.11 & 4.65 & 7.01 & 1.61 & 2.17 \\
\hline 40 & USW & USW & 25 & 68.0 & NA & NA & 0.10 & NA & 7.35 & NA & 2.50 \\
\hline
\end{tabular}


Table G-6: Data for Lee et al formula for selected buildings in Korea (from Lee et al (2000)) (...Continued)

\begin{tabular}{|c|c|c|c|c|c|c|c|c|c|c|c|}
\hline \multirow[b]{2}{*}{ ID } & \multicolumn{2}{|c|}{ Type } & \multirow{2}{*}{$\begin{array}{c}\text { Number of } \\
\text { storeys }\end{array}$} & \multirow[b]{2}{*}{ Height (m) } & \multirow[b]{2}{*}{$A_{B}\left(m^{2}\right)$} & \multicolumn{2}{|c|}{$L_{w}\left(m^{-1}\right)$} & \multicolumn{2}{|c|}{$h^{0.2} /\left(L_{w}{ }^{0.5}\right)$} & \multicolumn{2}{|c|}{ Measured period (s) } \\
\hline & Trans & Long & & & & Trans & Long & Trans & Long & Trans & Long \\
\hline 41 & USW & USW & 25 & 68.0 & NA & 0.21 & 0.13 & 5.07 & 6.45 & 1.69 & 2.13 \\
\hline 42 & USW & USW & 19 & 51.1 & NA & NA & 0.16 & NA & 5.49 & NA & 1.89 \\
\hline 43 & USW & USW & 20 & 53.9 & NA & 0.26 & 0.16 & 4.35 & 5.55 & 1.25 & 1.79 \\
\hline 44 & USW & USW & 15 & 40.0 & NA & 0.30 & 0.11 & 3.82 & 6.31 & 0.90 & 1.69 \\
\hline 45 & USW & USW & 20 & 55.6 & NA & NA & 0.19 & NA & 5.12 & NA & 1.79 \\
\hline 46 & USW & USW & 20 & 55.6 & NA & 0.17 & 0.16 & 5.42 & 5.58 & 1.25 & 1.72 \\
\hline 47 & USW & USW & 20 & 55.6 & NA & 0.25 & 0.14 & 4.47 & 5.97 & 1.27 & 1.82 \\
\hline 48 & USW & USW & 20 & 54.0 & NA & 0.28 & NA & 4.20 & NA & 1.25 & NA \\
\hline 49 & USW & USW & 20 & 54.0 & NA & 0.22 & 0.11 & 4.73 & 6.70 & 1.39 & 1.96 \\
\hline 50 & USW & USW & 20 & 54.0 & NA & 0.27 & 0.09 & 4.27 & 7.40 & 1.20 & 2.13 \\
\hline
\end{tabular}


Table G-7: Data for Morales formula for selected buildings in Montréal

\begin{tabular}{|c|c|c|c|c|c|c|c|c|c|c|c|}
\hline \multirow[b]{2}{*}{ ID } & \multicolumn{2}{|c|}{ Type } & \multirow{2}{*}{$\begin{array}{l}\text { Number of } \\
\text { Storeys }\end{array}$} & \multirow[b]{2}{*}{ Height (m) } & \multirow[b]{2}{*}{$A_{B}\left(m^{2}\right)$} & \multicolumn{2}{|c|}{$I\left(m^{4}\right)$} & \multicolumn{2}{|c|}{$\mathrm{h} /\left(\mathrm{I}^{0.25}\right)$} & \multicolumn{2}{|c|}{ Measured period (s) } \\
\hline & Trans & Long & & & & Trans & Long & Trans & Long & Trans & Long \\
\hline RCSW-1 & USW & CSW & 15 & 56 & 1310 & 137.00 & NA & 16.37 & NA & 0.99 & NA \\
\hline RCSW-2 & USW & CSW & 49 & 195 & 2599 & 188.39 & NA & 52.63 & NA & 4.35 & NA \\
\hline RCSW-6 & CSW & USW & 12 & 43 & 791 & NA & 33.01 & NA & 17.94 & NA & 0.77 \\
\hline RCSW-7 & CUSW & USW & 6 & 24 & 1327 & NA & 13.54 & NA & 12.51 & NA & 0.38 \\
\hline RCSW-8 & USW & CUSW & 6 & 23 & 1805 & 25.62 & NA & 10.22 & NA & 0.46 & NA \\
\hline RCSW-13 & USW & CUSW & 12 & 45 & 1723 & 6.40 & NA & 28.29 & NA & 0.99 & NA \\
\hline RCSW-16 & USW & USW & 6 & 20 & 809 & 8.53 & 13.89 & 11.70 & 10.36 & 0.40 & 0.36 \\
\hline RCSW-17 & CSW & USW & 22 & 75 & 2155 & NA & 154.42 & NA & 21.28 & NA & 1.75 \\
\hline RCSW-18 & USW & USW & 6 & 25 & 730 & 6.66 & 34.12 & 15.56 & 10.34 & 0.41 & 0.29 \\
\hline RCSW-20 & CSW & USW & 22 & 77 & 1709 & NA & 96.28 & NA & 24.58 & NA & 1.72 \\
\hline
\end{tabular}

CSW: Coupled shear walls

CUSW: Coupled and uncoupled shear walls

USW: Uncoupled shear walls

NA: Lateral loads not resisted by uncoupled shear walls

$A_{B}: \quad$ Building plan area at the base of the building

$\mathrm{I}: \quad$ Sum of moments of inertia of individual shear walls in the direction under consideration

h: Building height

Table G-8: Data for Morales formula for selected buildings in California (from Morales (2000))

\begin{tabular}{|c|c|c|c|c|c|c|c|c|c|c|c|}
\hline \multirow[b]{2}{*}{ ID } & \multicolumn{2}{|c|}{ Type } & \multirow{2}{*}{$\begin{array}{l}\text { Number of } \\
\text { Storeys }\end{array}$} & \multirow[b]{2}{*}{ Height (m) } & \multirow[b]{2}{*}{$A_{B}\left(m^{2}\right)$} & \multicolumn{2}{|c|}{$I\left(m^{4}\right)$} & \multicolumn{2}{|c|}{$\mathrm{h} /\left(\mathrm{I}^{0.25}\right)$} & \multicolumn{2}{|c|}{ Measured period (s) } \\
\hline & Trans & Long & & & & Trans & Long & Trans & Long & Trans & Long \\
\hline C24385 & USW & USW & 10 & 26.6 & 1687 & 164.178 & 164.307 & 7.43 & 7.43 & 0.57 & 0.62 \\
\hline C24468 & USW & USW & 8 & 38.5 & 2957 & 40.908 & 32.064 & 15.22 & 16.18 & 1.62 & 1.54 \\
\hline C24601 & USW & USW & 17 & 45.3 & 1687 & 282.967 & 193.201 & 11.04 & 12.15 & 1.05 & 1.18 \\
\hline C24655 & USW & USW & 6 & 18.5 & 7231 & 169.802 & 690.682 & 5.12 & 3.61 & 0.50 & 0.40 \\
\hline C12284 & USW & USW & 4 & 15.8 & 1003 & 13.657 & 12.434 & 8.22 & 8.41 & 0.60 & 0.50 \\
\hline C58334 & USW & USW & 3 & 11.0 & 754 & 12.5 & 12.5 & 5.85 & 5.85 & 0.18 & 0.18 \\
\hline C58348 & USW & USW & 3 & 12.3 & 937 & 7.479 & 17.402 & 7.44 & 6.02 & 0.46 & 0.38 \\
\hline C58394 & USW & USW & 9 & 31.5 & 1498 & 24.389 & 47.697 & 14.17 & 11.99 & 1.30 & 1.20 \\
\hline C58479 & USW & USW & 6 & 24.0 & 1118 & 243.249 & NA & 6.08 & NA & 0.22 & NA \\
\hline C57355 & USW & MRF & 10 & 37.5 & 1589 & 781.25 & NA & 7.09 & NA & 0.75 & NA \\
\hline C57356 & USW & USW & 10 & 29.0 & 1249 & 210.65 & 174.664 & 7.61 & 7.98 & 0.42 & 0.70 \\
\hline
\end{tabular}


Table G-8: Data for Morales formula for selected buildings in California (from Morales (2000)) (... Continued)

\begin{tabular}{|c|c|c|c|c|c|c|c|c|c|c|c|}
\hline \multirow[b]{2}{*}{ ID } & \multicolumn{2}{|c|}{ Type } & \multirow{2}{*}{$\begin{array}{c}\text { Number of } \\
\text { Storeys }\end{array}$} & \multirow[b]{2}{*}{ Height (m) } & \multirow[b]{2}{*}{$A_{B}\left(m^{2}\right)$} & \multicolumn{2}{|c|}{$I\left(m^{4}\right)$} & \multicolumn{2}{|c|}{$h /\left(1^{0.25}\right)$} & \multicolumn{2}{|c|}{ Measured period (s) } \\
\hline & Trans & Long & & & & Trans & Long & Trans & Long & Trans & Long \\
\hline C58344 & USW & USW & 1 & 10.0 & 1723 & 6.88 & 12.409 & 6.17 & 5.33 & 0.18 & 0.31 \\
\hline C47459 & USW & USW & 4 & 20.0 & 484 & 307.906 & 5.597 & 4.77 & 13 & 0.35 & 0.24 \\
\hline C13589 & USW & USW & 11 & 44.5 & 1086 & 527.056 & 4216.446 & 9.29 & 5.52 & 0.87 & 0.82 \\
\hline C23287 & USW & USW & 6 & 15.5 & 918 & 214.793 & 17.786 & 4.05 & 7.55 & 0.25 & 0.53 \\
\hline $\mathrm{C} 24517$ & USW & USW & 3 & 12.6 & 827 & 476.406 & 15.287 & 2.7 & 6.37 & 0.20 & 0.21 \\
\hline C14606 & USW & USW & 8 & 23.0 & 1117 & 82.05 & 95.118 & 7.64 & 7.36 & 0.55 & 0.68 \\
\hline C23285* & USW & USW & 5 & $10.0^{*}$ & $1723^{*}$ & $6.88^{*}$ & $12.409^{*}$ & $6.17^{*}$ & $5.33^{*}$ & 0.64 & 0.49 \\
\hline
\end{tabular}

${ }^{*}$ Most parameters identical to C58344 (values of $\mathrm{h} / \mathrm{l}^{0.25}$ are erroneous) 


\section{References}

Adams, J., and Atkinson, G. (2003). "Development of seismic hazard maps for the proposed 2005 edition of the National Building Code of Canada." Canadian Journal of Civil Engineering 30(2): 255-271.

Adams, J., and Halchuk, S. (2003). "Fourth generation seismic hazard maps of Canada: Values for over 650 Canadian localities intended for the 2005 National Building Code of Canada." Geological Survey of Canada Open File 4459, $155 \quad \mathrm{p}, \quad$ Available online at http://earthquakescanada.nrcan.gc.ca/.

Akaike, H. (1969a). "Fitting autoregressive models for prediction." Annals of the Institute of Statistical Mathematics 21: 243-247.

Akaike, H. (1969b). "Power spectrum estimation through autoregressive model fitting." Annals of the Institute of Statistical Mathematics 21: 407-419.

Ang, A. H. S., and Tang, W. H. (1975). Probability concepts in engineering planning and design, Wiley \& Sons, New York, NY.

ASCE. (2005). "Minimum design loads for buildings and other structures (ASCE/SEI 7-05)." Structural Engineering Institute, American Society of Civil Engineers, Reston, VA.

ATC. (1978). "Tentative provisions for the development of seismic regulations for buildings." Report No. ATC 3-06, Applied Technology Council, Palo Alto, CA.

Atkinson, G. M. (2009). "Earthquake time histories compatible with the 2005 National building code of Canada uniform hazard spectrum." Canadian Journal of Civil Engineering 36(6): 991-1000.

Atkinson, G. M., and Pierre, J.-R. (2004). "Ground-motion response spectra in Eastern North America for different critical damping values." Seismological Research Letters 75(4): 541-545.

Beck, J. L., May, B. S., Polidori, D. C., and Vanik, M. W. (1995). "Ambient vibration surveys of three steel-frame buildings strongly shaken by the 1994 Northridge earthquake." California Institute of Technology, Report No. EERL 95-06, Pasadena, California. 
Bendat, J. S., and Piersol, A. G. (2000). Random data: Analysis and measurement procedures, 3rd ed., John Wiley \& Sons, Inc., New York, N.Y., U.S.A.

Brincker, R., and Andersen, P. (1999). "Ambient response analysis modal analysis for large structures." Sixth International Congress on Sound and Vibration, Copenhagen, Denmark, $10 \mathrm{p}$.

Brincker, R., Ventura, C. E., and Andersen, P. (2001a). "Damping estimation by frequency domain decomposition." International Modal Analysis Conference - IMAC XIX, Kissimmee, FL, Feb. 2001, 698-703.

Brincker, R., Zhang, L., and Anderson, P. (2001b). "Modal identification of outputonly systems using frequency domain decomposition." Smart Materials and Structures 10: 441-445.

Brownjohn, J. M. W. (2003). "Ambient vibration studies for system identification of tall buildings." Earthquake Engineering and Structural Dynamics 32: 71-95.

Brownjohn, J. M. W., Lee, J., and Cheong, B. (1999). "Dynamic performance of a curved cable-stayed bridge." Engineering Structures 21: 1015-1027.

BSSC. (2003). "NEHRP recommended provisions for seismic regulations for new buildings and other structures (FEMA 450)." Building Seismic Safety Council, National Institute of Building Standards, Washington, D.C.

Calcagni Castillo, J. E. (1988). "Proposición de un espectro de diseño sísmico para la zona del terremoto del 3 de marzo de 1985." Thesis, Universidad de Chile, Santiago, Chile (In Spanish).

Carder, D. S. (1936). "Vibration observations, Chapter 5 in Earthquake investigations in California 1934-1935." U.S. Dept. of Commerce, Coast and Geologic Survey, Special Publication No. 201, Washington, D.C.

Çelebi, M. (1996). "Comparison of damping in buildings under low-amplitude and strong motions." Journal of Wind Engineering and Industrial Aerodynamics 59: 309-323.

Çelebi, M., and Liu, H.-P. (1998). "Before and after retrofit - Response of a building during ambient and strong motions." Journal of Wind Engineering and Industrial Aerodynamics 77: 259-268.

Chopra, A. K. (2001). Dynamics of structures: Theory and applications to earthquake engineering, 2nd ed., Prentice Hall, Upper Saddle River, NJ. 
Chopra, A. K., and Goel, R. K. (2000). "Building period formulas for estimating seismic displacements." Earthquake Spectra 16(2): 533-536.

Clinton, J. F., Bradford, S. C., Heaton, T. H., and Favela, J. (2006). "The observed wander of the natural frequencies in a structure." Bulletin of the Seismological Society of America 96(1): 237-257.

Clough, R. W., and Penzien, J. (2003). Dynamics of structures, 2nd ed. (revised), Computers and Structures, Inc., Berkeley, CA.

Computers and Structures, Inc. (2010). "SAP2000 (Version 14.2.0) [Software]."

Cooley, J. W., and Tukey, J. W. (1965). "An algorithm for the machine calculation of complex Fourier series." Mathematics of computation 19(90): 297-301.

Craig, R. R., and Kurdila, A. J. (2006). Fundamentals of Structural Dynamics, 2nd ed., John Wiley \& Sons, Hoboken, New Jersey, U.S.A.

Crawford, R., and Ward, H. S. (1964). "Determination of the natural periods of buildings." Bulletin of the Seismological Society of America 54(6): 17431756.

CTBUH. (2008). "Recommendations for the seismic design of high-rise buildings." Council on Tall Buildings and Urban Habitat, Chicago, Illinois, $21 \mathrm{p}$.

Darbre, G. R., De Smet, C. A. M., and Kraemer, C. (2000). "Natural frequencies measured from ambient vibration response of the arch dam of Mauvoisin." Earthquake Engineering and Structural Dynamics 29: 577586.

Ewins, D. J. (2000). Modal testing: Theory, practice and application, 2nd ed., Research Studies Press, Baldock, England.

Farrar, C. R., and James III, G. H. (1997). "System identification from ambient vibration measurements on a bridge." Journal of Sound and Vibration 205(1): 1-18.

Farsi, M. N., and Bard, P.-Y. (2004). "Estimation des périodes propres de bâtiments existants et vulnérabilité du bâti existant dans l'agglomération de Grenoble (France) [In French]." Revue Française de Génie Civil 8(2/3): 149-179.

Fritz, W. P., Jones, N. P., and Igusa, T. (2009). "Predictive models for the median and variability of building period and damping." Journal of Structural Engineering 135(5): 576-586. 
Gentile, C., and Gallino, N. (2008). "Ambient vibration testing and structural evaluation of an historic suspension footbridge." Advances in Engineering Software 39: 356-366.

Gersch, W., and Martinelli, F. (1979). "Estimation of structural system parameters from stationary and non-stationary ambient vibrations: An exploratoryconfirmatory analysis." Journal of Sound and Vibration 65(3): 303-318.

Gersch, W., Nielsen, N. N., and Akaike, H. (1973). "Maximum likelihood estimation of structural parameters from random vibration data." Journal of Sound and Vibration 31(3): 295-308.

Ghrib, F., and Mamedov, H. (2004). "Period formulas of shear wall buildings with flexible bases." Earthquake Engineering and Structural Dynamics 33: 295-314.

Gilles, D. (2008). "Evaluation of NBCC fundamental period formulae using ambient vibration data - Literature review of relevant topics." Report No. 2008-02, McGill University Structural Engineering Series, Montréal, QC, $56 \mathrm{p}$, Available online at http://www.mcgill.ca/library/libraryfindinfo/escholarship/.

Gilles, D. (2010). "In situ dynamic properties of buildings in Montréal determined from ambient vibration records." Report No. 2010-03, McGill University Structural Engineering Series, Montréal, QC, 170 p, Available online at http://www.mcgill.ca/library/library-findinfo/escholarship/.

Goel, R. K., and Chopra, A. K. (1997). "Vibration properties of buildings determined from recorded earthquake motions." Report No. UCB/EERC97/14, Richmond, CA.

Goel, R. K., and Chopra, A. K. (1998). "Period formulas for concrete shear wall buildings." Journal of Structural Engineering 124(4): 426-433.

Hans, S., Boutin, C., Ibraim, E., and Rousillon, P. (2005). "In situ experiments and seismic analysis of existing buildings. Part I: Experimental investigations." Earthquake Engineering and Structural Dynamics 34: 1513-1529.

Heidebrecht, A. (2003). "Overview of seismic provisions of the proposed 2005 edition of the National Building Code of Canada." Canadian Journal of Civil Engineering 30(2): 241-254. 
Hong, L.-L., and Hwang, W.-L. (2000). "Empirical formula for fundamental vibration periods of reinforced concrete buildings in Taiwan." Earthquake Engineering and Structural Dynamics 29: 327-337.

Huang, C. S., Hung, S. L., Wen, C. M., and Tu, T. T. (2003). "A neural network approach for structural identification and diagnosis of a building from seismic response data." Earthquake Engineering and Structural Dynamics 32: 187-206.

Humar, J. (2002). Dynamics of structures, 2nd ed., A. A. Balkema Publishers, Exton, PA.

Humar, J., and Mahgoub, M. A. (2003). "Determination of seismic design forces by equivalent static load method." Canadian Journal of Civil Engineering 30(2): 287-307.

ICC. (2006). "International Building Code." International Code Council, Country Club Hills, IL.

Ivanovic, S. S., Trifunac, M. D., Novikova, E. I., Gladkov, A. A., and Todorovska, M. I. (2000a). "Ambient vibration tests of a seven-story reinforced concrete building in Van Nuys, California, damaged by the 1994 Northridge earthquake." Soil Dynamics and Earthquake Engineering 19(6): 391-411.

Ivanovic, S. S., Trifunac, M. D., and Todorovska, M. I. (2000b). "Ambient vibration tests of structures - A review." ISET Journal of Earthquake Technology 37(4): 165-197.

Jeary, A. P. (1986). "Damping in tall buildings - A mechanism and a predictor." Earthquake Engineering and Structural Dynamics 14: 733-750.

Kadakal, U., and Yuzugullu, O. (1996). "A comparative study on the identification methods for the autoregressive modelling from the ambient vibration records." Soil Dynamics and Earthquake Engineering 15: 45-49.

Kijewski-Correa, T., and Pirnia, J. D. (2007). "Dynamic behavior of tall buildings under wind: Insights from full-scale monitoring." The Structural Design of Tall and Special Buildings 16: 471-486.

Lagomarsino, S. (1993). "Forecast models for damping and vibration periods of buildings." Journal of Wind Engineering and Industrial Aerodynamics 48: 221-239. 
Lamarche, C. P., Paultre, P., Proulx, J., and Mousseau, S. (2008). "Assessment of the frequency domain decomposition technique by forced-vibration tests of a full-scale structure." Earthquake Engineering and Structural Dynamics 37: 487-494.

Lee, L.-H., Chang, K.-K., and Chun, Y.-S. (2000). "Experimental formula for the fundamental period of RC buildings with shear-wall dominant systems." The Structural Design of Tall Buildings 9: 295-307.

Li, Y., and Mau, S. T. (1997). "Learning from recorded earthquake motion of buildings." Journal of Structural Engineering 123(1): 62-69.

Maia, N. M. M., Silva, J. M. M., He, J., Lieven, N. A. J., Lin, R. M., Skingle, G. W., To, W.-M., and Urgueira, A. P. V. (1997). Theoretical and experimental modal analysis, Research Studies Press, Baldock, England.

McVerry, G. H. (1979). "Frequency domain identification of structural models from earthquake records." Report No. EERL 79-02, California Institute of Technology, Pasadena, California, 213 p.

Memari, A. M., Aghakouchak, A. A., Ashtiany, M. G., and Tiv, M. (1999). "Fullscale dynamic testing of a steel frame building during construction." Engineering Structures 21: 1115-1127.

Midorikawa, S. (1990). "Ambient vibration tests of buildings in Santiago and Viña del Mar." Pontifica Universidad Católica de Chile, Escuela de Ingeniería, Departamento de Ingeniería Estructural, Santiago, Chile.

Mitchell, D., Paultre, P., Tinawi, R., Saatcioglu, M., Tremblay, R., Elwood, K., Adams, J., and DeVall, R. (2010). "Evolution of seismic design provisions in the National building code of Canada." Canadian Journal of Civil Engineering 37: 1157-1170.

Mitchell, D., Tremblay, R., Karacabeyli, E., Paultre, P., Saatcioglu, M., and Anderson, D. L. (2003). "Seismic force modification factors for the proposed 2005 edition of the National Building Code of Canada." Canadian Journal of Civil Engineering 30(2): 308-327.

Morales, M. D. (2000). "Fundamental period of vibration for reinforced concrete buildings." Master's thesis, University of Ottawa, Ottawa, ON.

Newmark, N. M., and Rosenblueth, E. (1971). Fundamentals of earthquake engineering, Prentice Hall, Englewood Cliffs, NJ. 
NRC/IRC. (2005a). "National Building Code of Canada 2005." National Research Council of Canada, Institute for Research in Construction, Ottawa, ON.

NRC/IRC. (2005b). "User's Guide - NBC 2005 Structural Commentaries (Part 4 of Division B)." National Research Council of Canada, Institute for Research in Construction, Ottawa, ON.

Oppenheim, A. V., and Schafer, R. W. (1999). Discrete-time signal processing, Prentice-Hall, Upper Saddle River, NJ.

Pan, T.-C., You, X., and Brownjohn, J. M. W. (2006). "Effects of infill walls and floor diaphragms on the dynamic characteristics of a narrow-rectangle building." Earthquake Engineering and Structural Dynamics 35: 637-651.

Parloo, E., Guillaume, P., and Cauberghe, B. (2003). "Maximum likelihood identification of non-stationary operational data." Journal of Sound and Vibration 268(5): 971-991.

PEER/ATC. (2010). "Modeling and acceptance criteria for seismic design and analysis of tall buildings." Report No. PEER/ATC-72-1, Applied Technological Council (ATC) in collaboration with the Pacific Earthquake Engineering Research (PEER) Center, Redwood City, California.

Proulx, J., Paultre, P., Rheault, J., and Robert, Y. (2001). "An experimental investigation of water level effects on the dynamic behaviour of a large arch dam." Earthquake Engineering and Structural Dynamics 30: 11471166.

Rainieri, C., Fabbrocino, G., and Cosenza, E. (2010). "Some remarks on experimental estimation of damping for seismic design of civil constructions." Shock and Vibration 17: 383-395.

Saatcioglu, M., and Humar, J. (2003). "Dynamic analysis of buildings for earthquake-resistant design." Canadian Journal of Civil Engineering 30(2): 338-359.

Satake, N., Suda, K.-i., Arakawa, T., Sasaki, A., and Tamura, Y. (2003). "Damping evaluation using full-scale data of buildings in Japan." Journal of Structural Engineering 129(4): 470-477.

Schiff, A. J. (1972). "Identification of large structures using data from ambient and low level excitations." in System Identification of Vibrating Structures, W. D. Pilkey and R. Cohen, eds., ASME, New York, NY.

Schott, J. R. (2005). Matrix Analysis for Statistics, Wiley \& Sons, Hoboken, NJ. 
SEAOC. (1999). "Recommended lateral force requirements and commentary (SEAOC Blue Book)." Seismology Committee, Structural Engineers Association of California, Sacramento, CA.

Structural Vibration Solutions A/S. (2009a). "ARTeMIS Extractor Handy (Version 4.2) [Software]." Available online at http://www.svibs.com.

Structural Vibration Solutions A/S. (2009b). "User's guide to ARTeMIS Extractor Handy (Version 4.2)."

Structural Vibration Solutions A/S. (2010). "Operational Modal Analysis Software." http://www.svibs.com/. Accessed on September 25, 2010.

The Mathworks, Inc. (2008). "MATLAB (Version 7.7.0.471 (R2008b)) [Software]."

Trifunac, M. D. (1972). "Comparisons between ambient and forced vibration experiments." Earthquake Engineering and Structural Dynamics 1: 133150.

Trifunac, M. D., Ivanovic, S. S., and Todorovska, M. I. (2001a). "Apparent periods of a building. I: Fourier analysis." Journal of Structural Engineering 127(5): 517-526.

Trifunac, M. D., Ivanovic, S. S., and Todorovska, M. I. (2001b). "Apparent periods of a building. II: Time-frequency analysis." Journal of Structural Engineering 127(5): 527-537.

Udwadia, F. E., and Trifunac, M. D. (1974). "Time and amplitude dependent response of structures." Earthquake Engineering and Structural Dynamics 2: 359-378.

Van Overschee, P., and De Moor, B. (1993). "Subspace algorithms for the stochastic identification problem." Automatica 29(3): 649-660.

Ventura, C. E., and Schuster, N. D. (1996). "Structural dynamic properties of a reinforced concrete high-rise building during construction." Canadian Journal of Civil Engineering 23: 950-972.

Wallace, J. W., and Moehle, J. P. (1992). "Ductility and detailing requirements of bearing wall buildings." Journal of Structural Engineering 118(6): 16251644.

Yang, J. N., Lei, Y., Pan, S., and Huang, N. (2003). "System identification of linear structures based on Hilbert-Huang spectral analysis. Part 1: Normal modes." Earthquake Engineering and Structural Dynamics 32: 14431467. 
Zar, J. H. (1999). Biostatistical analysis, 4th ed., Prentice Hall, Upper Saddle River, New Jersey. 